\title{
Targeted Quantitative Lipidomics of Cold Stress and the Development of Methods to Increase the Sensitivity of Proteomics Analyses using Mass Spectrometry
}

\author{
by
}

Katrin Blank

A thesis submitted to the Faculty of Graduate and Postdoctoral Affairs in partial fulfillment of the requirements for the degree of

Doctor of Philosophy

in

Chemistry with Specialization in Chemical and Environmental Toxicology

Carleton University

Ottawa, Ontario

(C) 2018

Katrin Blank 


\section{Abstract}

This thesis focuses on mass spectrometry (MS) based techniques and uses them to discover new lipidomic profiles of species that adapt to cold temperatures in the northern climates. Additionally, techniques were developed to enhance MS-based proteomics analyses for better protein identification. This research looks at both qualitative and quantitative analysis techniques: the focus in the lipidomic work is mainly on relative quantification, while the proteomics work enhances qualitative analyses.

A lipid bilayer is of interest and will be examined in the tissues of several animal models. The composition of lipid bilayers is investigated in three different cold stress adaptation mechanisms including hibernation, freeze tolerance and freeze avoidance. Also examined are lipid bilayer differences in cold adaption in vital versus non-vital organs. The research was also conducted on how certain seasonal rhythms are preserved even in the absence of environmental cues. The study models that were used were the thirteen-lined ground squirrel for hibernation, the wood frog for freeze tolerance and the goldfish for freeze avoidance traits. Our results elucidate some exciting patterns of lipid bilayers in adaptation to cold stress. Increases in the concentration of unsaturated phospholipids in cold temperatures, particularly in the squirrel and frog liver tissues were observed. Also, in some cases increases in phosphatidylethanolamine lipids were observed in the lipid bilayers during winter months in comparison to summer months. These biomolecular dynamics are linked with increases in the fluidity of the lipid bilayer which is necessary for a continued physiological function at lower temperatures. 
Proteomics work was focused further developments of the Trimethylation Enhancement using Diazomethane (TrEnDi) technique. Diazomethane was used to methylate tryptically digested peptides or commercial peptides. TrEnDi derivatization allows for the formation of fixed permanent charges on the peptides making them more sensitive in MS analyses. These results also highlight a novel method to identify the phosphorylation of peptides, which holds a significant interest in a great deal of clinical and health-based research as dysfunctional phosphorylation pathways are linked to numerous diseases. Although TrEnDi derivatization requires further optimization on peptide samples at this point in time, the developments described herein demonstrate that it is a unique method that can enhance the sensitivity of MS-based peptide analysis in numerous ways. 


\section{Acknowledgments}

These four years just flew by, and I am about to start a new chapter in my life, I feel scared and excited at the same time. I think these four years have been good years though of course challenging. I do not want to move on without appreciating everyone who has helped me along the way and got me here.

Thank you, Jeffrey Smith, you have been a great supervisor. You have played the most significant role in getting me where I am today. Thank you for believing in me and accepting me under your wing four years ago. It always has been straightforward to communicate with you. You have repeatedly encouraged me and provided me with everything I needed to do this research.

I appreciate all the help and support that you have provided me, Carlos Canez, with your knowledge in mass spectrometry and lipidomics you have been a valuable resource. I also appreciate that I was able to text you almost any time of the day and night and I was able to get all the answers I needed. I wish you all the best in your future studies.

I want to say thank you to Sam Shields who has helped me with many organic syntheses and provided me with encouragements to work with diazomethane. Thank you for your braveness. Also, Hillary Weinert, I am happy I had a chance to meet you. I had a great time working with you in summer 2016. Thank you for helping me with a lot of tedious diazomethane titration work and for just being a great company.

Thank you, Dr. Kenneth Storey and Dr. Michael Jonz, without you most of this thesis would not be possible. The wood frog tissues, as well as the ground squirrel liver 
tissue, came from Dr. Storey laboratory. Dr. Jonz was able to give me some goldfish retina tissue for some lipidomics research.

As always my biggest supporters are my family - mom and Selvan, without your constant love and support I would not have the energy and drive to get where I am today. I am looking forward spending more time with you both. Thank you for all your patience. 


\section{Table of Contents}

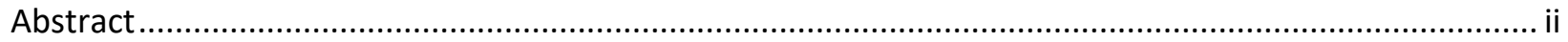

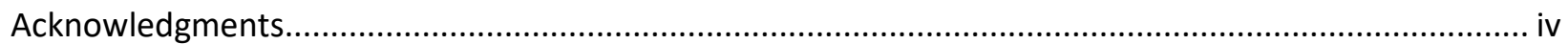

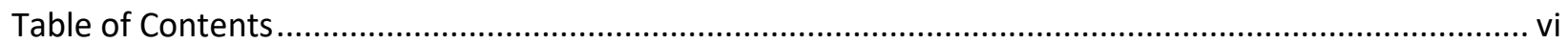

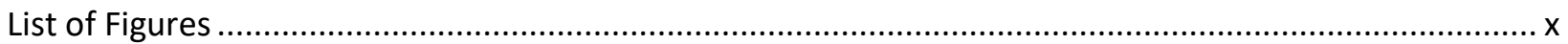

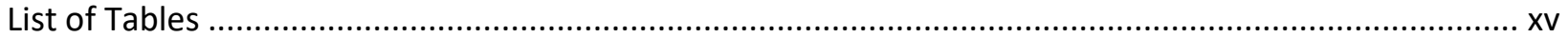

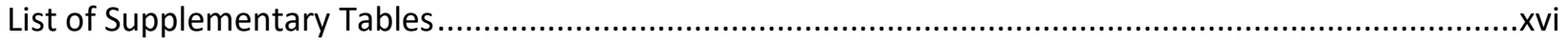

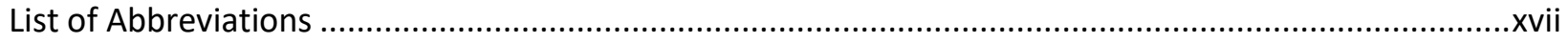

Chapter 1. What role do phospholipids play in the adaption of eukaryotes to survive cold climates?.1

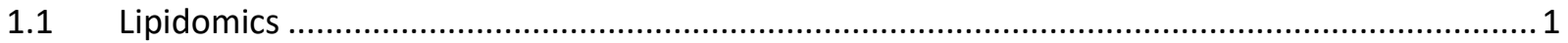

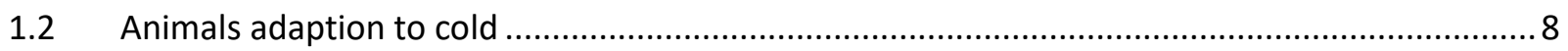

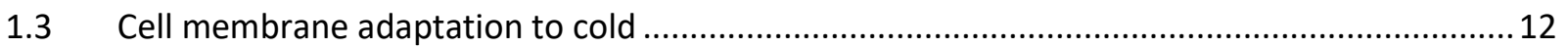

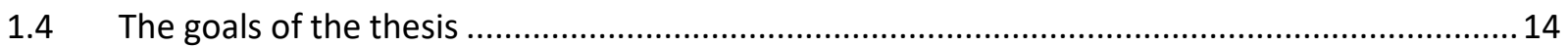

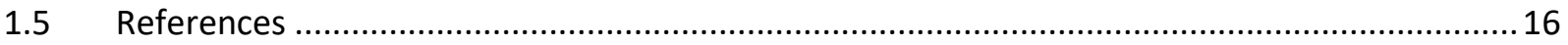

Chapter 2. Mass spectrometry instruments and high-performance liquid chromatography separation

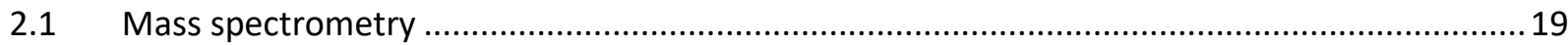

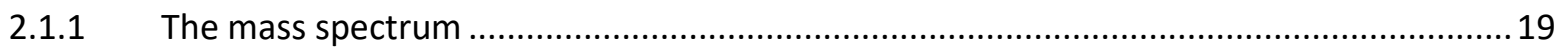

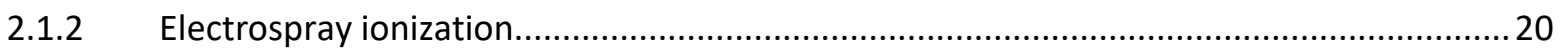

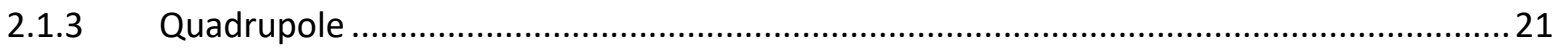

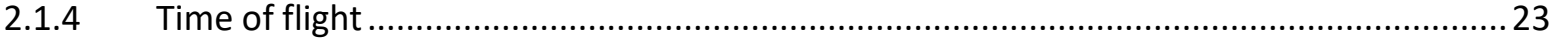

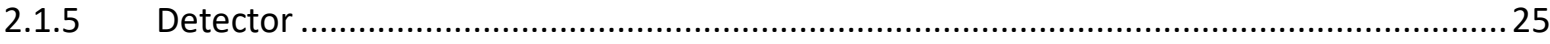

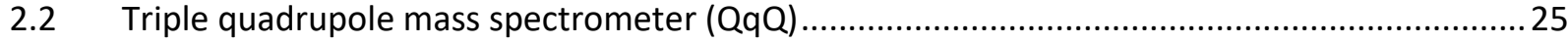

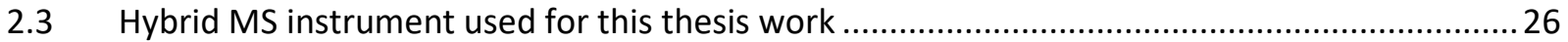

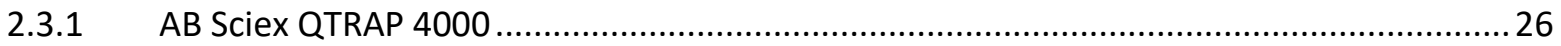

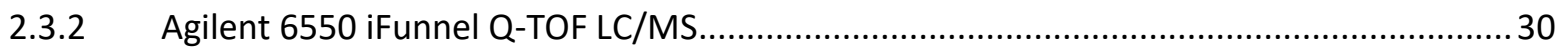

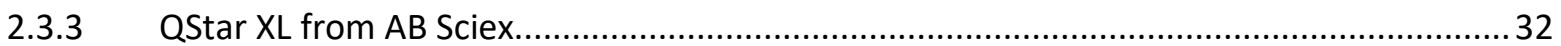

2.4 Separation via high-performance liquid chromatography ...................................................3

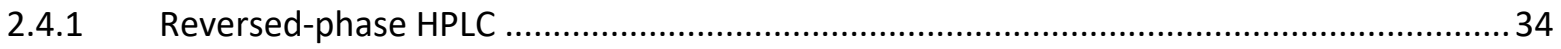

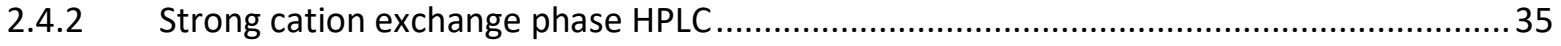

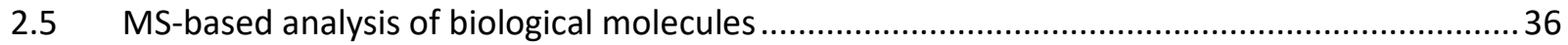

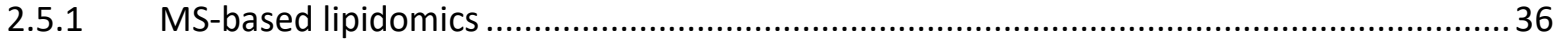




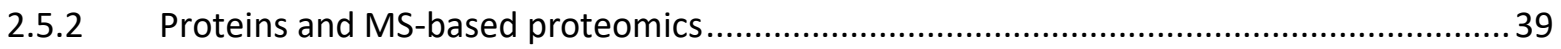

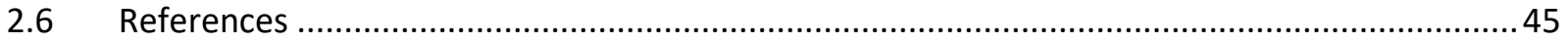

Chapter 3. Investigation of phospholipid structural dynamics between euthermic and hibernating

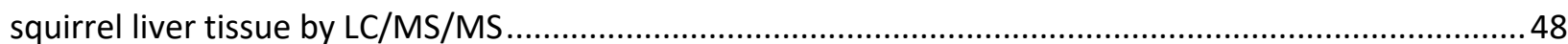

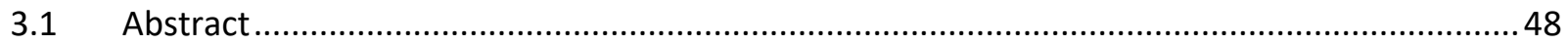

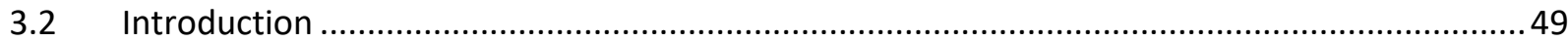

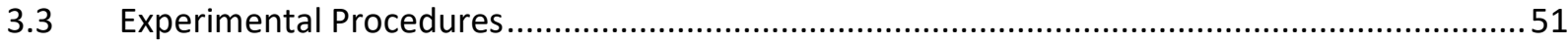

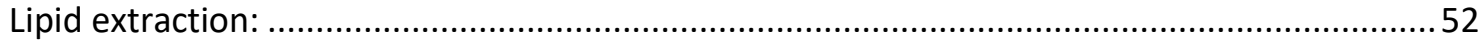

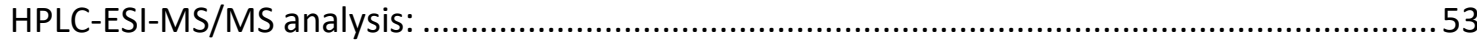

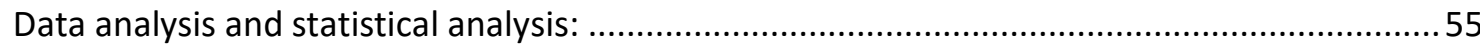

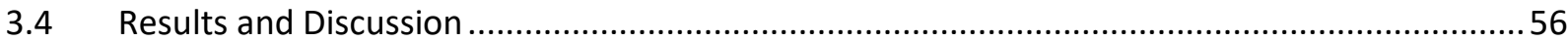

3.4.1 Triple quadrupole linear ion trap quantitation ............................................................60

3.4.2 Q-TOF structural identification using high-resolution $\mathrm{m} / \mathrm{z}$ values..................................65

3.4.3 Fatty acid chain identification via negative ESI MS/MS experiments..............................69

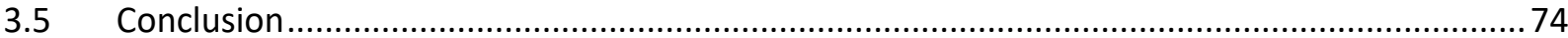

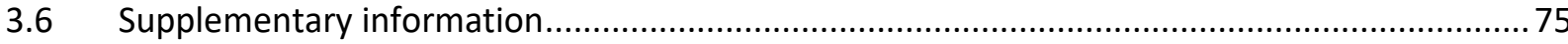

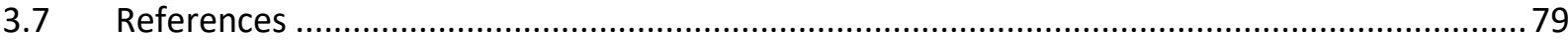

Chapter 4. Analysis of glycerophospholipid dynamics in Rana sylvatica leg muscle and liver tissue during extreme temperature fluctuations via HPLC-MS/MS ................................................................ 81

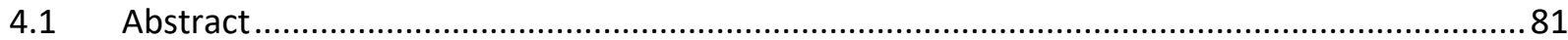

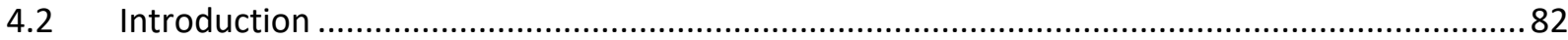

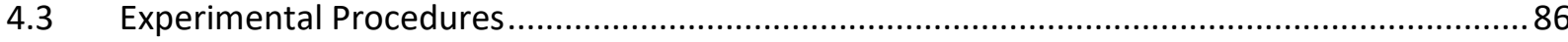

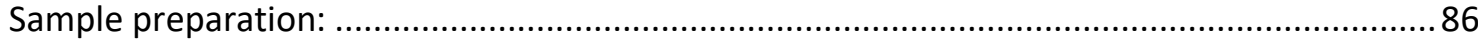

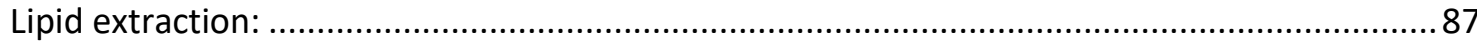

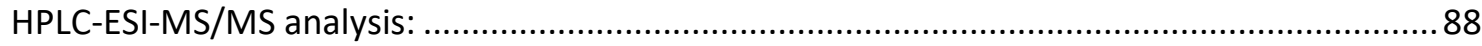

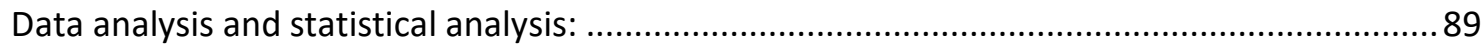

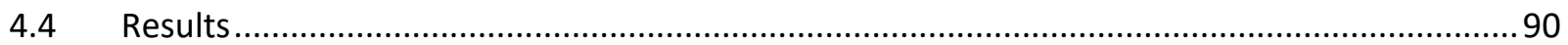

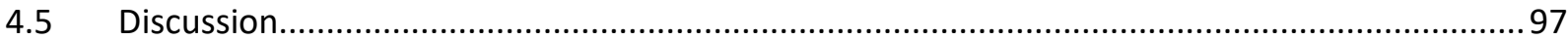

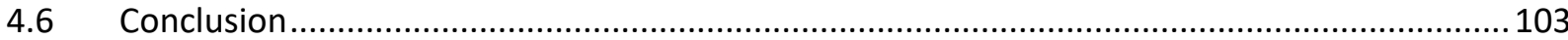

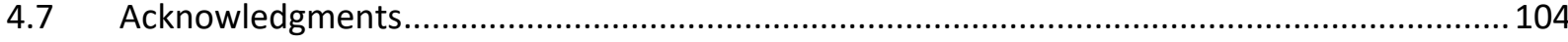

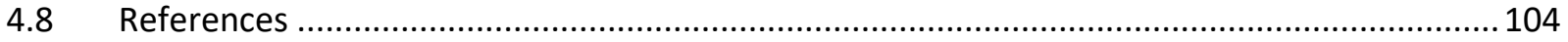

Chapter 5. Using MS-based lipidomics to better understand the curious circannual rhythms in

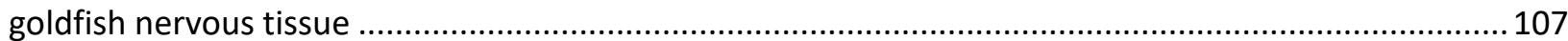

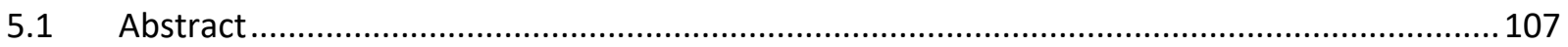




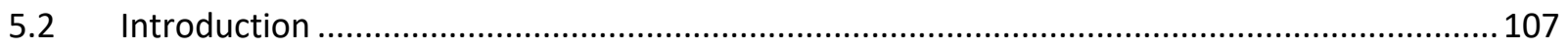

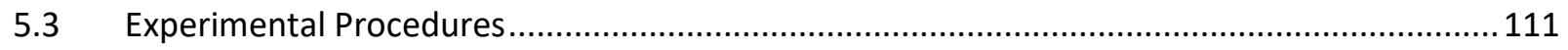

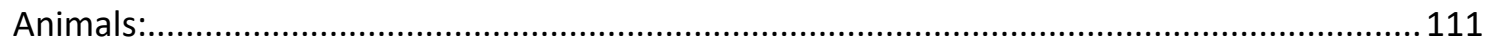

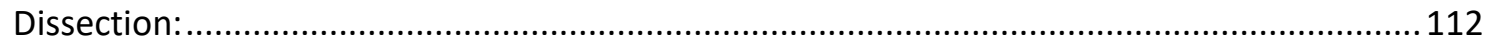

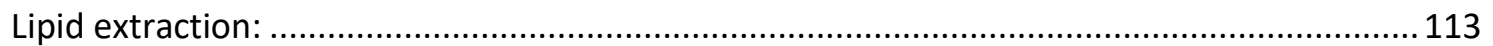

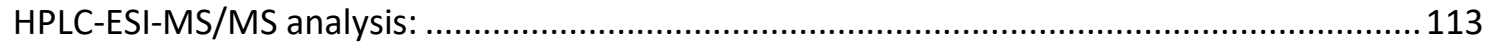

Selective functional group derivatization reactions: ........................................................... 115

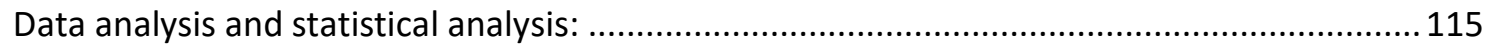

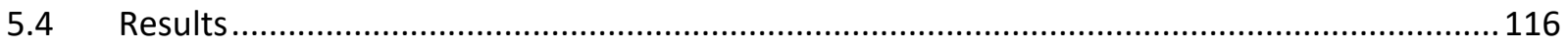

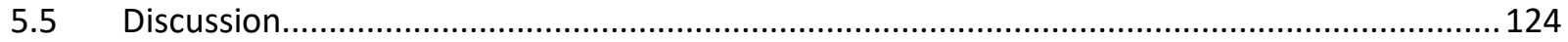

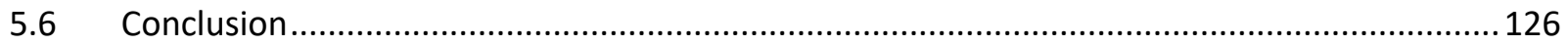

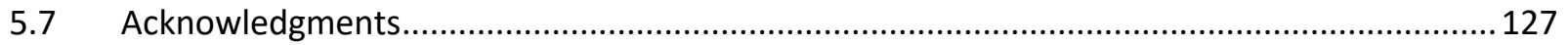

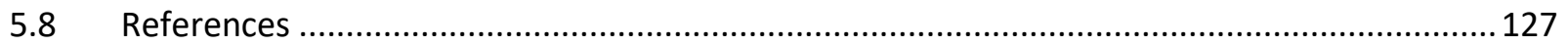

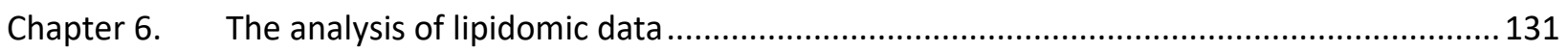

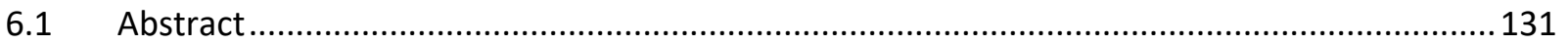

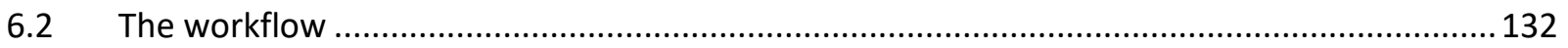

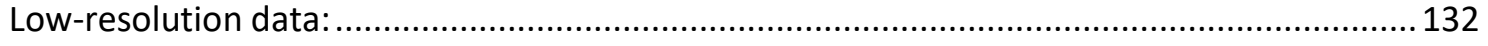

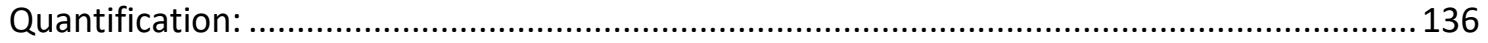

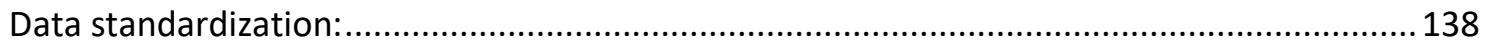

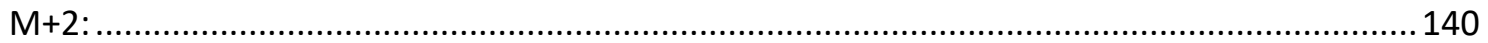

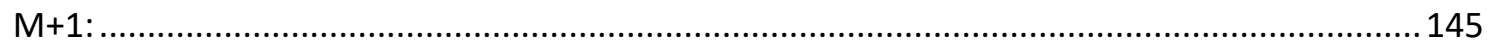

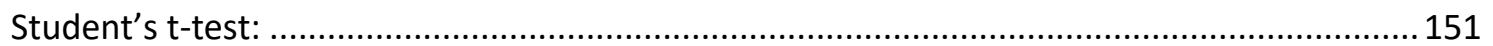

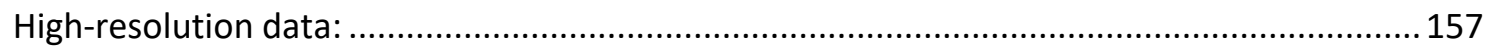

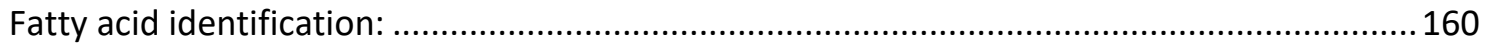

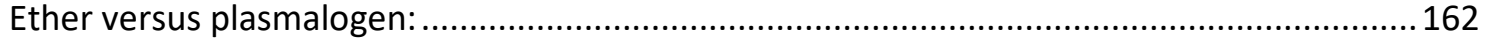

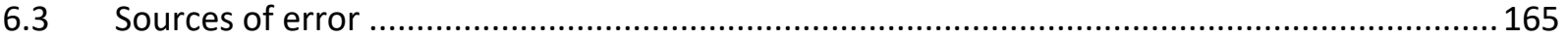

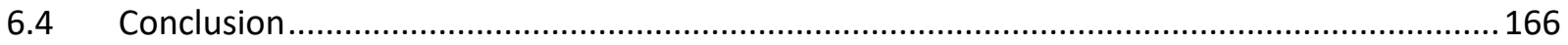

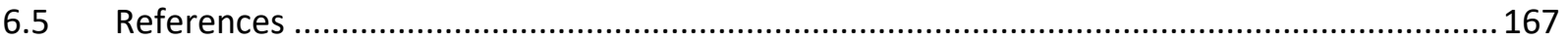

Chapter 7. The development of methods to simplify MS spectra for better identification of peptides

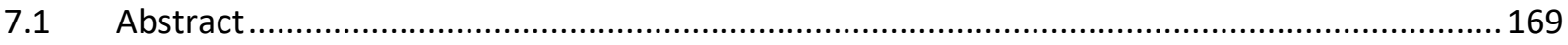

7.2 Diazomethane and methylation of commercial peptides ................................................. 170

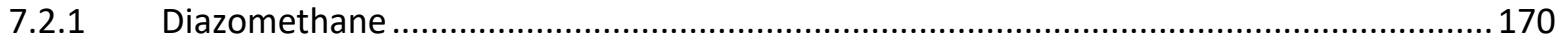


7.2.1.1 Synthesis via N-Methyl-N-nitroso-urea (NMU).

7.2.1.2 Synthesis via and N-Methyl-N-nitroso-p-toluenesulfonamide (Diazald).

7.2.1.3 Diazomethane titrations .....

7.2.2 HPLC separation of TrEnDi modified peptides............................................................. 176

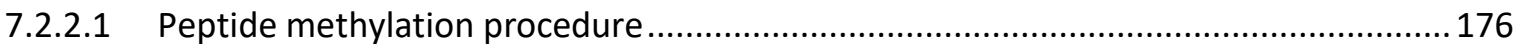

7.2.2.2 Elution of peptides from SCX column and separation of TrEnDi modified peptides on HPLC 179

7.2.3 TrEnDi to unify the charge state on methylated peptides............................................ 185

7.2.4 Phosphorylated peptides using TrEnDi ....................................................................... 193

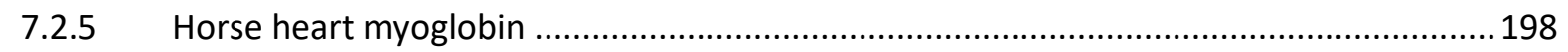

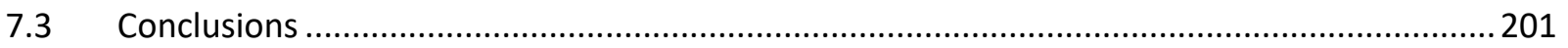

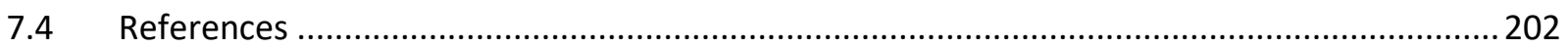




\section{List of Figures}

Figure 1:1. Structures of lipid classes (phospholipids not shown here) ................................................ 2

Figure 1:2. Structures of phospholipid classes: glycerophospholipids (PEs, PCs, PSs and PIs) and

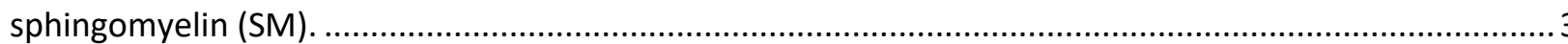

Figure 1:3. Cell membrane diagram. This Figure was adapted from https://biologydictionary.net/cellmembrane/

Figure 1:4. Examples of birds that migrate to warmer climates to avoid cold winter. Examples on the photos are from left to right: Canada goose, warbler, red-headed woodpecker and duck..... 9 Figure 1:5. Characteristics of freeze avoidance (through supercooling) and freeze avoidance. This Figure was adapted from Costanzo and Lee, $2013^{37}$

Figure 1:6. Molecular structures of soybean and coconut oils. This Figure was adapted from Costa et al., $2016^{46}$.

Figure 1:7. Visual representation of two kinds of the lipid bilayers. On the right, phospholipid fatty acyl chains are saturated (no double bonds) making the tails tightly packed making bilayer more viscous; on the left, phospholipid fatty acyl chains are unsaturated (has double bonds) making the tails have kinks making the bilayer more fluid. This Figure was adapted from http://ib.bioninja.com.au/standardlevel/topic-1-cell-biology/13-membrane-structure/membrane-fluidity.html.

Figure 1:8. Shape and structure of phosphatidylcholine $(P C)$ and phosphatidylethanolamine (PE). This Figure was adapted from Zalba et al., $2017^{58}$.....

Figure 2:1. ESI mass spectrum of protonated caffeine. This Figure was adapted from Schwartz et al., $2002^{2}$.

Figure 2:2. Mechanism of electrospray ionization. This Figure was adapted from Demartini, $2013^{7} \ldots \ldots .21$

Figure 2:3. Schematic of a quadrupole analyzer. This Figure was adapted from http://www.bris.ac.uk/nerclsmsf/techniques/gcms.html.

Figure 2:4. Schematic of reflectron TOF-MS. This Figure was adapted from http://elchem.kaist.ac.kr/vt/chem-ed/ms/tof.htm

Figure 2:5. Schematic of a triple quadrupole (QqQ) analyzer. This Figure was adapted from https://www.biologie.hu-berlin.de/de/gruppenseiten/oekologie/meth/massspec/mass_sp.....

Figure 2:6. Schematic of a hybrid triple quadrupole linear ion trap instrument. This Figure was adapted from Cox et al., $2005^{20}$.

Figure 2:7. Schematic diagram of multiple reaction monitoring. This Figure was adapted from Colangelo et al., $2013^{22}$.

Figure 2:8. Schematic of Agilent iFunnel Q-TOF LC/MS (with major improvements to other instruments circled by Agilent). This Figure was adapted from the manual for the $6550^{23}$.

Figure 2:9. ESI in 6550 iFunnel Q-TOF LC/MS system. This Figure was adapted from Manual for $6550^{23} .31$

Figure 2:10. Hexabore capillary and dual stage ion funnel. This Figure was adapted from Manual for $6550^{23}$

Figure 2:11. Schematic of a Q-TOF mass spectrometer. This Figure was adapted from Smith, $2005^{24} \ldots . .33$

Figure 2:12. Visual representation of the gradient used in the phospholipid experiments. ....................36 Figure 2:13. Typical workflow of MS-based lipidomics. This Figure was adapted from Khalil et al., $2010^{25}$.

Figure 2:14. Amino acid structures. This Figure was adapted from https://en.wikipedia.org/wiki/Proteinogenic_amino_acid..... 
Figure 2:15. Peptide bond formation when two amino acids are combined.

Figure 2:16. Horse heart myoglobin protein amino acid sequence, colors indicate peptides observed after trypsin digestion.

Figure 2:17. An example of peptide fragmentation locations and nomenclature. This Figure was adapted from Wysocki et al., $2005^{38}$.

Figure 2:18. The typical workflow in MS-based proteomics. (1) Samples under analysis are fractioned to obtain a proteomic mixture which is separated (SDS-Page is one example of separation techniques) and proteins of interest are cut out from SDS-Page. (2) Protein of interest is digested to peptide fragments (trypsin is a common enzyme used for this procedure). (3) Peptide sample mix obtained is subject to MS and MS/MS experiments on Q-TOF instrument. (4 and 5) MS and MS/MS spectra are obtained and analyzed for peptides and ultimately for protein identification. This Figure was adapted from Aebersold and Mann, $2003^{39}$.

Figure 3:1. Total ion chromatogram of one of the control sample replicates acquired via reversed-phase LC-ESI-MS. Lysoglycerophospholipids and other polar lipids are the first ones to elute. Most diradyl GPLS elute between $18 \mathrm{~min}$ and $22 \mathrm{~min}$. Many diacylglycerols and most of the triacylglycerol species elute between minute 22.5 and minute 27 . The predominant lipid species present in the spectrum are GLs despite many GPLs having better ionization efficiencies.

Figure 3:2. Panel A presents the extracted ion chromatogram (EIC) of 758 Th PC acquired via a PIS of 184 Th, from a torpor replicate. The PIS of 184 Th enabled the exclusive quantitation of PC (34:2) without isobaric interference. Panel B presents the EIC of two eluting PEs with $\mathrm{m} / \mathrm{z}$ of 758 acquired. The EIC was acquired using NL scan of $141 \mathrm{Da}$ from a torpor replicate and enabled the identification and exclusive quantitation of the two PE species. The peak with a retention time (Rt) of 22.09 min corresponds to PE (38:9), and the peak with an Rt of 25.59 min corresponds to PE (37:2). Panel C overlays the elution profiles of the PIS 184 Th and NL of $141 \mathrm{Da}$. The PC (34:2) peak has the biggest area of all PC species in the sample. Both PE areas are slightly above their respective LoQ. Panel $\mathrm{C}$ demonstrates that it would have been impossible to identify or quantify PE (37:2) with a high-resolution MS since it shares the same $\mathrm{m} / \mathrm{z}$ of 758.5694 as the dwarfing PC. The PE (38:9) would have been able to be identified as it has a different mass (758.4755 Th).

Figure 3:3. The six PC chromatograms were obtained using PIS of 184 Th. The top three spectra are representative EICs from control samples, while the bottom three spectra are representatives of torpor samples. The left column portrays EICs of 772 Th; the middle column shows EICs of 790 Th, and the right column portrays EICs of $820 \mathrm{Th}$. In all three examples, the earlier eluting peaks are significantly upregulated during torpor. Two different brand new in-house columns were used for the control and torpor triplicates. Thus there was an Rt shift that can be observed in the spectra. Using the Rt of the internal standard, corrected retention times (cRts) for all phospholipids were calculated. The average cRt for both control 772 Th peaks are 9.0 and $11.8 \mathrm{~min}$, while the torpor counterparts were $9.0 \mathrm{~min}$ and $11.9 \mathrm{~min}$. The average cRt for both control 790 Th peaks are 7.6 and $12.9 \mathrm{~min}$; the torpor counterparts were $7.6 \mathrm{~min}$ and $12.9 \mathrm{~min}$. The average cRt for both control 820 Th peaks are 9.3 and $12.2 \mathrm{~min}$; the torpor counterparts were $8.9 \mathrm{~min}$ and $12.2 \mathrm{~min}$.

Figure 3:4. Negative ESI product ion scan spectra of three odd-chained statistically significant PC species. The product ion scan for PC (33:2) [802.5597 Th] in panel A demonstrates the presence of 279.2290 Th (C18:2) and 241.2144 Th (C15:0), as well as 255.2293 Th (C16:0) and 265.2127 Th (C17:2). Panel B shows the product ion scan for PC (35:3) [828.5749 Th] where 279.2298 Th (C18:2) and 267.2294 Th (C17:1), as well as 281.2459 Th (C18:1) and 265.2152 Th (C17:2) are present. Panel C shows the product ion scan for 
PC (35:1) [832.6062 Th] where 281.2467 Th (C18:1) and 269.2461 Th (C17:0), as well as 283.2606 Th (C18:0) and 267.2300 Th (C17:1) are present.

Figure 4:1. (a) The generic structure of a glycerophospholipid; R1 and R2 are two nonpolar aliphatic chains (brown box), the core of the molecule is glycerol (blue box), a phosphate bound to a polar head group X (red box). Glycerol group carbons from the top are sn-1, sn-2, and sn-3 (b) Structure of sphingomyelin; 18-carbon amino alcohol (sphingosine) (black box) binds to a phosphocholine head group (red box) and a fatty acid group (green box). (c) head groups that are investigated in this work. . 86 Figure 4:2. Relative distribution of glycerophospholipids found in wood frog liver and leg muscle samples. There are a total of 195 phospholipids found in wood frog liver samples above the limit of quantification (LOQ), and a total of 122 phospholipids found in wood frog leg samples above the LOQ. 92 Figure 4:3. Phospholipid concentrations are compared between control and dehydrated wood frog liver tissues. The bottom panel reveals the distribution after applying the false discovery rate correction using Benjamini-Hochberg (BH) method ( $\alpha=0.01)$.

Figure 4:4. Phospholipid concentrations are compared between control and dehydrated wood frog leg muscle tissues. The bottom panel reveals the distribution after applying a false discovery rate correction using Benjamini-Hochberg $(\mathrm{BH})$ method $(\alpha=0.01)$.

Figure 4:5. Relative abundances of $24 \mathrm{PC}$ lipids in wood frog liver control versus dehydrated samples that change in a statistically significant manner after applying the $\mathrm{BH}$ correction.

Figure 5:1. Schematic representation of a human eye on the left and enlarged retinal tissue on the right $^{16}$.

Figure 5:2. Relative abundances of glycerophospholipids and sphingomyelins found in goldfish eye retina samples. There are a total of 153 phospholipids found in goldfish eye retina samples above the limit of quantification (LOQ).

Figure 5:3. Phospholipid concentrations are compared between January and July goldfish eye retina tissues. The phospholipid concentration could be not changing, increasing/decreasing, appearing/disappearing in the July tissue when compared to the January tissue. The bottom panel shows the same comparison after a false discovery rate correction using the Benjamini-Hochberg $(\mathrm{BH})$ method $(\alpha=0.15)$.

Figure 5:4. High-resolution MS extracted ion chromatograms from five PEs that decrease in concentration in summer months compared to winter months in goldfish retinal tissue. These chromatograms allow for the determination of the exact masses of the PEs of interest (indicated in the title of each chromatogram).

Figure 5:5. Possible structures of nominal mass $\mathrm{m} / \mathrm{z} 688 \mathrm{PEs}$ after fatty acid chain identification and determination of relative proportions of ethereal-acyl to plasmalogen-acyl species. These PE structures are also found in the lipid database of Lipidomics Gateway. Many other possible structures are possible; this Figure does not consider sn-1 and sn-2 locations of the fatty acid chains as well the location and the configuration of the double bonds (though $\mathrm{Z}$ configurations of the double bond are the most common ones)

Figure 6:1. $\mathrm{m} / \mathrm{z} 184$ is the precursor fragment for PC and SM; $141 \mathrm{Da}$ is the neutral loss fragment for PE; $185 \mathrm{Da}$ is the neutral loss fragment for PS 132

Figure 6:2. The TIC versus time chromatogram is shown in above chromatogram. The below chromatogram shows PC peaks extracted between 24 to 25 minutes from TIC versus time. 134 
Figure 6:3. The TIC versus time chromatogram is shown in above chromatogram. The below chromatograms show SM (m/z 184 precursor ion scan), PE (141 Da neutral loss scan) and PS (185 Da neutral loss scan) peaks extracted between 24 to 25 minutes from TIC versus time.

Figure 6:4. The peak list that is extracted for PCs from intensity versus $\mathrm{m} / \mathrm{z}$ chromatogram between 24 to 25 minutes. This peak list data can be extracted as a text file.

Figure 6:5. MultiQuant 2.1.1. software view. Shown here are area extractions for 820 PE peak for January and July goldfish retina sample in triplicates.

Figure 6:6. Isotopic distribution of $\mathrm{PC}(13: 0 / 0: 0)$ lipid. The Figure was generated via the Lipidomics Gateway lipid database.

Figure 6:7. $M+2$ calculations for goldfish retina membrane PE species in January done in Excel. ..........143

Figure 6:8. $M+2$ calculations for goldfish retina membrane PE species in July done in Excel. ................ 144 Figure 6:9. $M+1$ calculations for goldfish retina membrane $P C$ and SM species in January done in Excel.

Figure 6:10. $P C M+1$ contribution subtraction from SM peaks.

Figure 6:11. Areas and retention times as well as standardized areas and retention times of all the PE species found in goldfish retina membranes.

Figure 6:12. Averages of standardized areas and retention times of all the PE species found in goldfish retina membranes, also standard deviation values and \% standard deviation values are shown. .154

Figure 6:13. Fold changes and $p$-values at $\alpha=0.05$ between January and July of all the PE species found in goldfish retina membranes. 155

Figure 6:14. Benjamini-Hochberg (BH) correction of goldfish retina membranes significant $\mathrm{PEs}$ at $\alpha=$ $0.15(\alpha=Q)$. 158

Figure 6:15. Internal standard peak from high-resolution data. 159

Figure 6:16. Spectrum preview shows MS/MS spectrum for ion 686.4756 Th....................................162

Figure 6:17. The whole peak list extracted for MS-MS spectrum for ion 686.4756 Th......................... 163 Figure 6:18. Excel sheet is showing proportions of ethereal-acyl and plasmalogen-acyl species for two PE

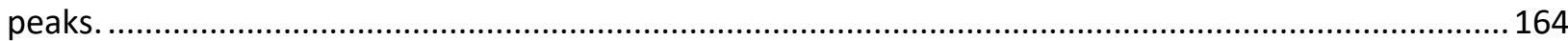

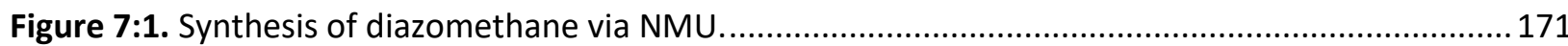

Figure 7:2. Synthesis of diazomethane via Diazald. ......................................................................... 172

Figure 7:3. High pressure vessel used for column chemistry. Image above shows the vessel set up with column, image below shows solvent compartment where vials are inserted.

Figure 7:4. Loading peptides on an SCX column in the first step. Methylating peptides on the SCX column by running ethereal diazomethane through the peptide loaded column in the second step. ...............179

Figure 7:5. TEAA salt is prepared from equimolar quantities of triethylamine and acetic acid..............181

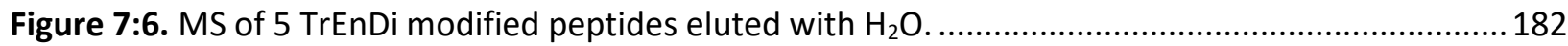

Figure 7:7. Loading TrEnDi modified peptides on a new SCX column..............................................183

Figure 7:8. Eluting TrEnDi modified peptides with 200 mM TEAA solution. FLEEHK appears in a lot smaller intensity compared to the other peptides (not shown here) ................................................ 184

Figure 7:9. Eluting TrEnDi modified peptides over 30 minutes using a $0-200 \mathrm{mM}$ TEAA gradient. ...... 185

Figure 7:10. Unmodified $\mathrm{H}_{3} \mathrm{~K}_{4}$ histone peptides doubly charged. ................................................. 188

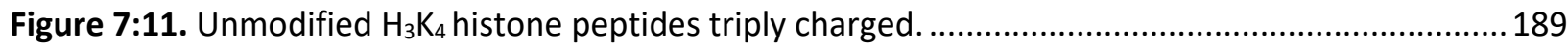

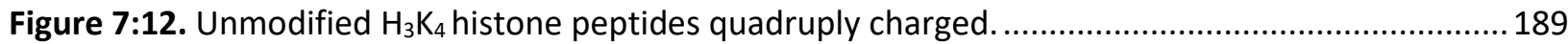

Figure 7:13. Unmodified $\mathrm{H}_{3} \mathrm{~K}_{4}$ histone peptides quintuply charged............................................... 190

Figure 7:14. TrEnDi modified $\mathrm{H}_{3} \mathrm{~K}_{4}$ histone peptides quintuply charged............................................ 190 
Figure 7:15. Unmodified $\mathrm{H}_{3} \mathrm{~K}_{9}$ histone peptides doubly charged.

Figure 7:16. Unmodified $\mathrm{H}_{3} \mathrm{~K}_{9}$ histone peptides triply charged. ................................................191

Figure 7:17. Unmodified $\mathrm{H}_{3} \mathrm{~K}_{9}$ histone peptides quadruply charged............................................ 192

Figure 7:18. TrEnDi modified $\mathrm{H}_{3} \mathrm{~K}_{4}$ histone peptides quadruply charged...........................................192

Figure 7:19. Three amino acids that can be phosphorylated through the phosphoester bond formation.

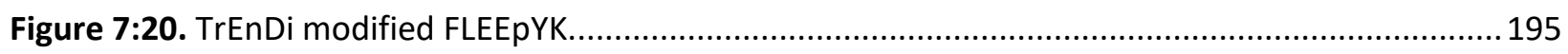

Figure 7:21. TrEnDi modified FLEEpYK - MS/MS 531....................................................

Figure 7:22. TrEnDi modified FLEEpSK............................................................................. 196

Figure 7:23. TrEnDi modified FLEEpSK - MS/MS 493.......................................................... 196

Figure 7:24. TrEnDi modified FLEEpTK.......................................................................... 197

Figure 7:25. TrEnDi modified FLEEpTK - MS/MS 500............................................................. 197

Figure 7:26. MASCOT sequence coverage for $4 \mu \mathrm{g}$ of TrEnDi modified myoglobin peptides methylated with $250 \mu \mathrm{L}$ of $0.2 \mathrm{M}$ diazomethane solution. Data obtained by Q-TOF instrument.............................200 Figure 7:27. MASCOT sequence coverage for trypsin digested horse heart myoglobin. Data obtained by 6550 instrument. 200 


\section{List of Tables}

Table 2:1. Shown are various triple quadrupole linear ion trap operation modes. This table was adapted from Hopfgartner et al., 2004 ${ }^{21}$.

Table 2:2. Identification of phospholipids using tandem MS. This table is modified table 2 from Bandu et al., $2016^{28}$

Table 3:1. Statistically significant PC, SM, PE and PS peaks after student's t-test and BH false discovery rate correction.

Table 3:2. High-resolution $\mathrm{m} / \mathrm{z}$ values obtained via positive ESI and negative ESI using a Q-TOF instrument.

Table 3:3. Radyl information for the primary and secondary PC, PE and PS species obtained from negative ESI MS/MS experiments using a Q-TOF instrument. .70

Table 4:1. PC lipids that increase in response to freezing temperatures in the wood frog liver. .98

Table 4:2. PC lipids that do not increase in response to freezing temperatures in the wood frog liver....99 Table 4:3. Relative percentages and ratios of phospholipids in the liver and leg muscle membranes from wood frog tissues between control and dehydration conditions.

Table 4:4. Number of double bonds found in 24 significant and 24 most non-significant PCs out of 88 total quantifiable PCs in wood frog liver tissue membranes. This table uses data from tables 4:1 and 4:2 and assumes diacyl structure for all the fatty acid chains. 102

Table 5:1. Significant PEs that decrease during the seasonal change from January to July. ...................119 Table 5:2. Identified PEs with exact mass data and relative abundances.

Table 6:1. LOD and LOQ values calculated by Canez et al., 2016 by measuring 90 limit of blank along with 90 low concertation standard deviations as described in Armbruster and Pry, 2008. PIS stands for precursor ion scan, and NLS stands for neutral loss scan.

Table 6:2. The ratio values of total percentage phospholipids and of masses of dehydrated versus control tissues of wood frog liver and leg muscle tissues and July versus January tissues of goldfish retina tissue.

Table 7:1. Diazomethane concentrations when it is produced from NMU precursor and either $50 \%$ or $45 \% \mathrm{KOH}$ solutions. Reaction time was 7 minutes in each case. Concentration values via two titration methods are compared

Table 7:2. Diazomethane concentration values. The reaction of NMU with $50 \% \mathrm{KOH}$ was carried out during a 20-minute period, small amounts of sample were taken at specific time points and analyzed for diazomethane concentration; two separate batches were made at two different dates. Diazald was also used to make diazomethane on two different dates, however, in these cases, three trials of each batch were sampled and analyzed for concentration.

Table 7:3. Shown are pKa values for amino acid chains (obtained from chapter 2 amino acid table) and TrEnDi modification of these amino acids. This table is the modified version from Wasslen et al., 2014 (Supporting Information) ${ }^{4}$. The rest of the amino acid chains are not shown as all of their side chain pKa values are above $12^{4}$.

Table 7:4. Commercial peptides with their neutral masses and $\mathrm{m} / \mathrm{z}$ ratios before and after TrEnDi modification. The table also shows a total number of methylations expected and obtained as well as a fixed number of positive charges.

Table 7:5. ARTKQTARK peptides in MS spectra before and after TrEnDi.......................................... 186

Table 7:6. QTARKSTGGK peptides in MS spectra before and after TrEnDi. 


\section{List of Supplementary Tables}

Table S. 3:1. Non-statistically significant PC peaks after student t-test analysis and Benjamini-Hochberg

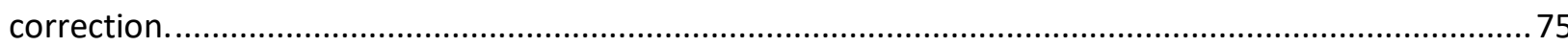

Table S. 3:2. Non-statistically significant SM peaks after student t-test analysis and Benjamini-Hochberg correction.

Table S. 3:3. Non-statistically significant PE peaks after student t-test analysis and Benjamini-Hochberg correction.

Table S. 3:4. Non-statistically significant PS peaks after student t-test analysis and Benjamini-Hochberg correction. 


\section{List of Abbreviations}

\begin{tabular}{ll}
$\%$ & percentage \\
$\mu \mathrm{m}, \mathrm{cm}$ & micrometer, centimeter \\
${ }^{19} \mathrm{~F}$ & fluorine-19 \\
$2 \mathrm{D}$ & two-dimensional \\
ABC & ammonium bicarbonate \\
AC & alternating current \\
AcMeOH & acetic acid in methanol \\
ACN & acetonitrile \\
ACUC & Animal Care and Use Committee \\
ACVS & Animal Care and Veterinary Services \\
ADC & analog-to-digital converter \\
ADP & adenosine diphosphate \\
ATP & adenosine triphosphate \\
BH & Benjamini-Hochberg \\
BLoD & below limit of detection \\
BLoQ & below limit of quantification \\
BSA & bovine serum albumin \\
Cat. No & catalog number \\
CCAC & Canadian Council on Animal Care \\
CHCA & a-cyano-4-hydroxycinnamic acid \\
CID & collision-induced dissociation \\
Conc. & concentration \\
cRT & corrected retention time \\
C-terminus & carbon terminus \\
d & deuterium label \\
Da, kDA & Dalton, kiloDalton \\
DC & direct current \\
Dehyd & dehyded \\
\hline &
\end{tabular}




\begin{tabular}{ll} 
DHB & dihydroxybenzoic acid \\
DMAPP & dimethylallyl diphosphate \\
DNA & deoxyribonucleic acid \\
Dr & Doctor \\
e.g. & for example \\
EIC & extracted-ion chromatogram \\
ESI & electrospray ionization \\
et al. & and others \\
EtOH & ethanol \\
FA & fatty acyl or fatty acid \\
FA & formic acid \\
Fig & Figure \\
FWER & familywise error rate \\
g, $\mu g$ & gram, microgram \\
GAA & glacial acidic acid \\
GL & glycerolipids \\
GPL & glycerophospholipids \\
h & hour \\
HC & liquid chromatography \\
HEPES & horizontal cell \\
HPLC & 4-(2-hydroxyethyl)-1-piperazineethanesulfonic acid \\
ID & high-performance liquid chromatography, high-pressure \\
Inc & iquid chromatography \\
IPA & internal diameter \\
IPP & Isoproporation \\
K & lysine \\
kV & kilovolt \\
L, mL, $\mu$ LL, mL & liter, milliter, microliter, nanoliter \\
LC & licohol \\
\hline
\end{tabular}




\begin{tabular}{|c|c|}
\hline LoD, LOD & limit of detection \\
\hline LoQ, LOQ & limit of quantification \\
\hline LPC & lysophosphatidylcholine \\
\hline M & molecular mass \\
\hline $\mathrm{m} / \mathrm{z}$ & mass-to-charge \\
\hline MA & Massachusetts \\
\hline MALDI & matrix-assisted desorption ionization \\
\hline $\min$ & minute \\
\hline $\mathrm{mM}$ & millimolar \\
\hline MRM & multiple reaction monitoring \\
\hline MTBE & methyl tert-butyl ether \\
\hline MS & mass spectrometry \\
\hline NINDS & National Institute of Neurological Disorders and Stroke \\
\hline NJ & New Jersey \\
\hline NL & neutral loss \\
\hline NMR & nuclear magnetic resonance \\
\hline NMU & $N$-methyl- $N$-nitroso-urea \\
\hline NSERC & Natural Sciences and Engineering Research Council \\
\hline N-terminus & nitrogen terminus \\
\hline O & radyl \\
\hline O & ethereal \\
\hline OAc & acetate \\
\hline${ }^{\circ} \mathrm{C}$ & Celsius \\
\hline ON & Ontario \\
\hline$P$ & proline \\
\hline$P$ & plasmalogen \\
\hline PA & phosphatidic acid \\
\hline PBS & phosphate-buffered saline \\
\hline PC & phosphatidylcholine, precursor ion \\
\hline
\end{tabular}




$\begin{array}{ll}\text { PE } & \text { phosphatidylethanolamine } \\ \text { PFI } & \text { phospholipid fluidity index } \\ \text { PG } & \text { phosphatidylglycerol } \\ \text { pH } & \text { potential of hydrogen } \\ \text { PI } & \text { phosphatidylinositol, product ion } \\ \text { PIC } & \text { precursor ion scan } \\ \text { PK } & \text { polyketides } \\ \text { pKa } & \text { acid dissociation constant } \\ \text { ppm } & \text { part per million } \\ \text { PR } & \text { prenol lipids } \\ \text { PS } & \text { phosphatidylserine } \\ \text { Q } & \text { quadruple } \\ \text { QqQ } & \text { triple quadruple } \\ \text { R } & \text { arginine } \\ \text { RF } & \text { radio frequency } \\ \text { rpm } & \text { revolutions per minute } \\ \text { Rt } & \text { retention time } \\ \text { S } & \text { second } \\ \text { S } & \text { supplemental } \\ \text { SCX } & \text { strong cation exchange } \\ \text { SL } & \text { saccharolipids } \\ \text { SM } & \text { sphingomyelin } \\ \text { sn-1, sn-2, sn-3 } & \text { stereospecific numbering-1, stereospecific numbering-2, } \\ \text { SRM } & \text { stereospecific numbering-3 } \\ \text { ST } & \text { selected reaction monitoring } \\ \text { Tb } & \text { sterol lipids } \\ \text { TEAA } & \text { body temperature } \\ \text { Th } & \text { triethylammonium acetate } \\ \text { TLC } & \end{array}$




$\begin{array}{ll}\text { TLS } & \text { 13-lined squirrel } \\ \text { TOF } & \text { time of flight } \\ \text { TrEnDi } & \text { Trimethylation Enhancement using Diazomethane } \\ \text { U } & \text { unit } \\ \text { USA } & \text { United States of America } \\ \text { WAT } & \text { white adipose tissue } \\ X & \text { times } \\ \text { XIC } & \text { extracted-ion chromatogram }\end{array}$




\section{Chapter 1. What role do phospholipids play in the adaption of eukaryotes to survive cold climates?}

\subsection{Lipidomics}

Lipidomics is a subsection of metabolomics and is a field that investigates the complete lipid profile within a cell, tissue, organism, or ecosystem. Lipidomics research focuses on identification and quantification of lipid species as well as their interaction with other lipids, proteins and other metabolites (sugars, nucleotides and amino acids). The primary biological functions of lipids are energy storage, lipid signaling, as well they are major structural components of cell membranes ${ }^{1,2}$. Lipids' importance is also recognized in various lipidomics diseases, such as diabetes ${ }^{3}$, obesity ${ }^{4}$, stroke ${ }^{5}$, hypertension ${ }^{6}$, and atherosclerosis ${ }^{7}$.

Fahy et al., 2005 classifies lipids into eight groups depending on their chemical structure: fatty acids, glycerolipids, glycerophospholipids, sphingolipids, sterol lipids, prenol lipids, saccharolipids, and polyketides ${ }^{8}$. These different structures of lipids are shown in Figure 1:1 and Figure 1:2.

Fatty acids constitute one of the essential categories of biological lipids, and they are also building blocks of more complex lipids. There are many structural variations of fatty acid chains, but the most basic ones are straight-chain saturated fatty acids with the terminal carboxylic acid group. Different structures of fatty acids can be obtained with the addition of unsaturation, or attachment of various functional groups with oxygen, nitrogen, halogen, and sulfur, or even cyclic structures could be part of the chain. In the unsaturated 


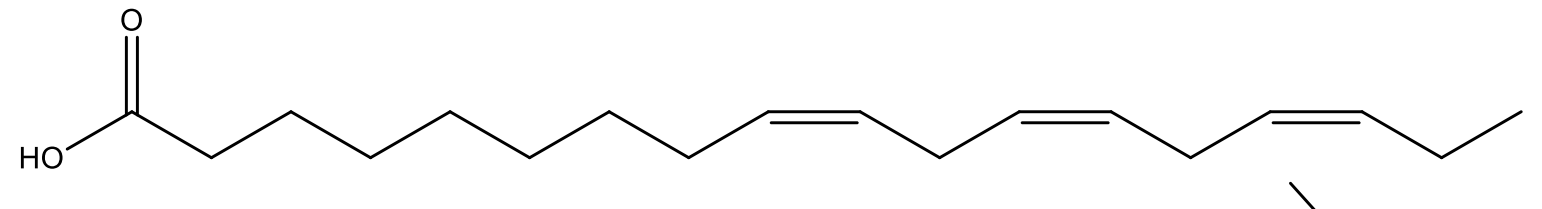

Class: Fatty acyls (FA)<smiles>[R]C(=O)OCC(COC([R])=O)OC([R2])=O</smiles>

Class: Glycerolipids (GL)

Name: Triacylglycerols

Name: a-Linolenic acid

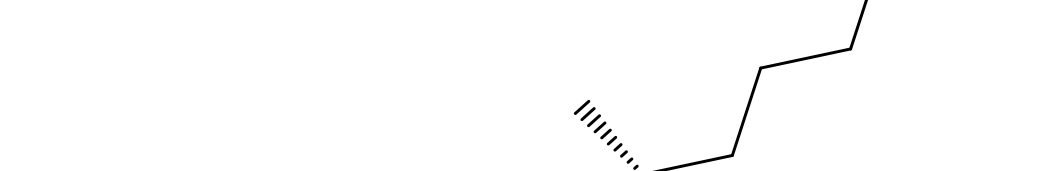<smiles>CC(C)=CCC/C(C)=C/CO</smiles>

Class: Prenol lipids (PR)

Name: 2E-geraniol

Sterol lipids (ST)

Name: Cholesterol

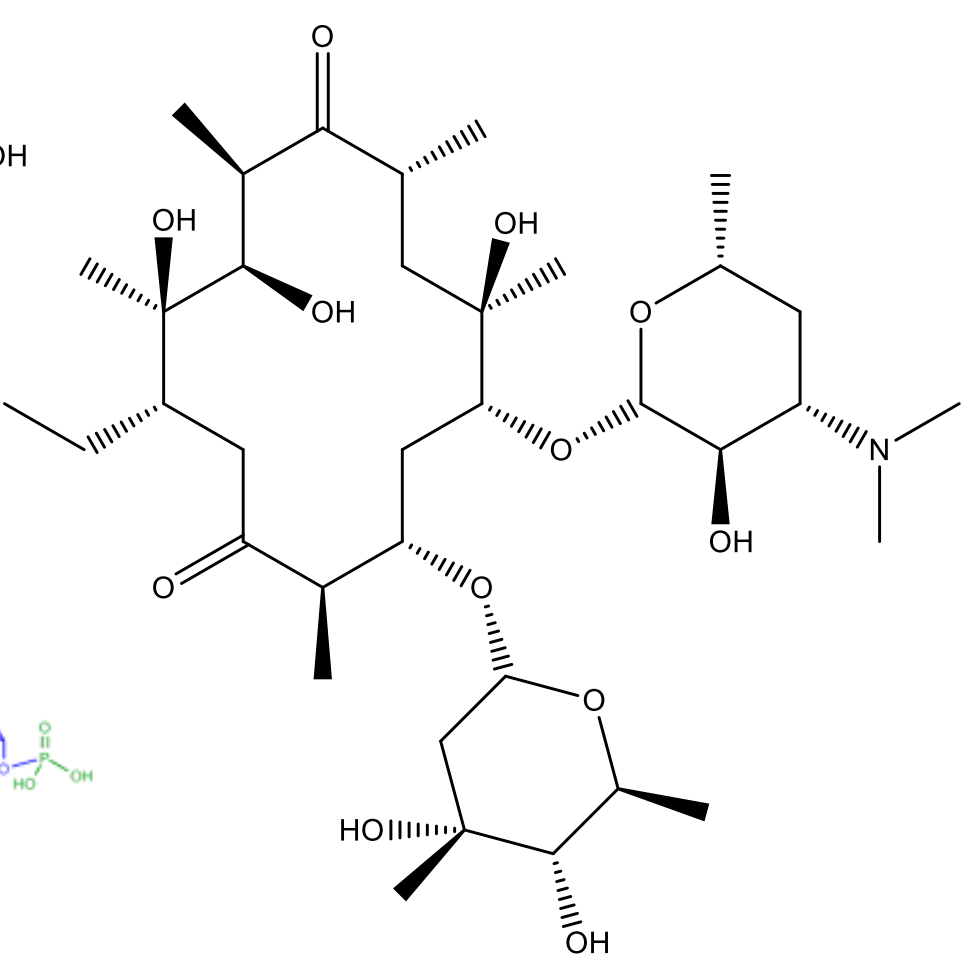

Class: Saccharolipids (SL)

Name: Kdo2-Lipid A

Class: Polyketides (PK)

Name: Erythromycin C

Figure 1:1. Structures of lipid classes (phospholipids not shown here). 


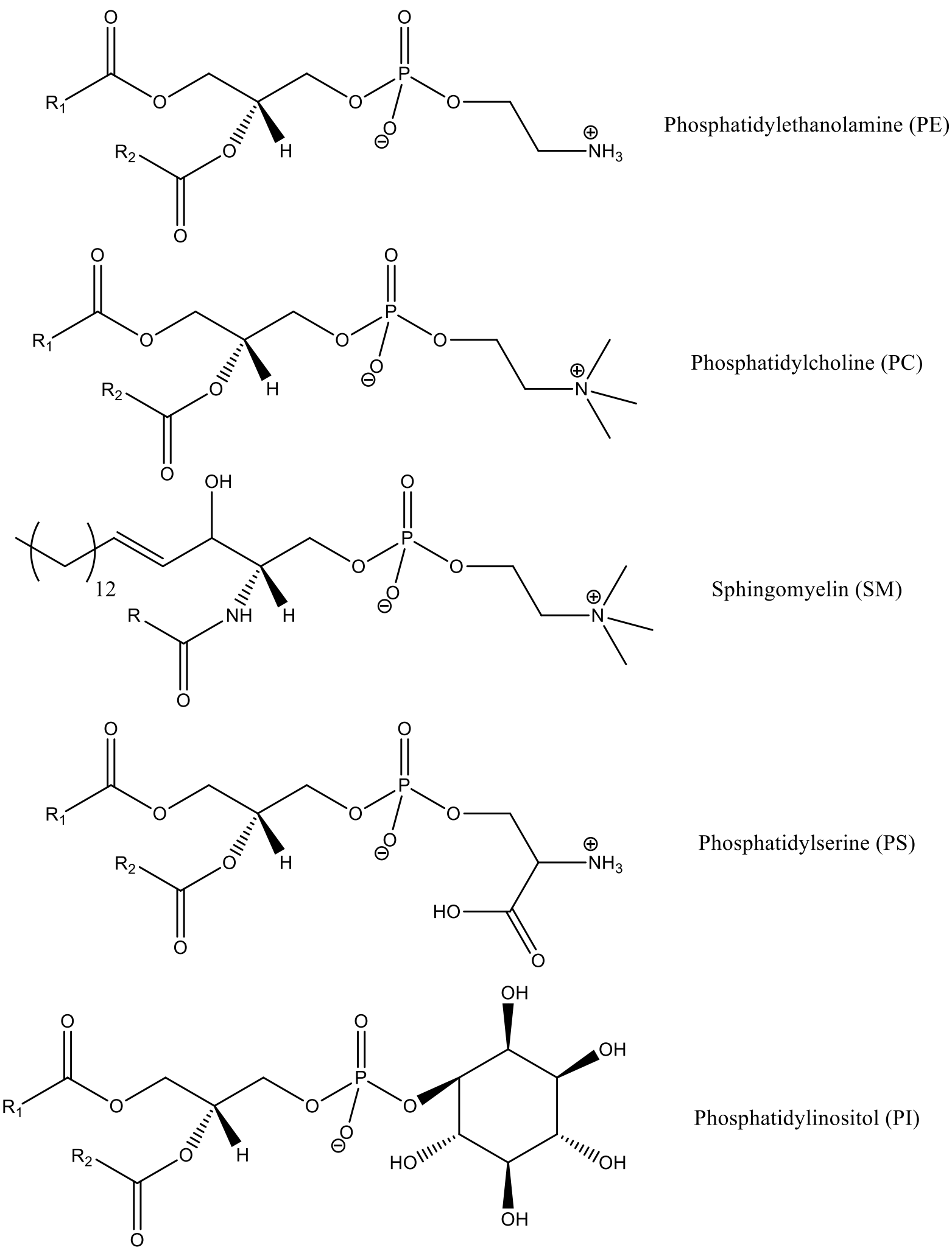

Figure 1:2. Structures of phospholipid classes: glycerophospholipids (PEs, PCs, PSs and PIs) and sphingomyelin (SM). 
fatty acids the cis configuration of the double bonds is more common in naturally occurring fatty acids ${ }^{9}$.

Glycerolipids contain a glycerol backbone and can be either mono-, di-, or trisubstituted. The primary function of glycerolipids is to act as energy storage units; they are readily found in animal tissues as stored fat (fat storage tissue is called adipose tissue). In the initial step of fat metabolism, fatty acids and glycerol are released through the hydrolysis of ester bonds of the glycerolipids ${ }^{10}$.

Sterol lipids (also called steroids) have the same fused four-ring core structure as shown in Figure 1:1 for cholesterol. Cholesterol and its derivatives are the most studied among sterol lipids; they are vital elements found in the cell membranes. Cholesterol is considered important molecule that affects membrane fluidity; where usually more cholesterol in membranes is associated with less fluidity of those membranes ${ }^{11,12}$. However, some studies have shown that membrane fluidity might be not the essential function of cholesterol ${ }^{13,14}$ and like other steroids, they act mainly in lipid signaling by activating steroid hormonal receptors ${ }^{15,16}$.

The general structure for prenol lipids is $\mathrm{H}-\left[\mathrm{CH}_{2} \mathrm{CCH}_{3}=\mathrm{CHCH}_{2}\right] \mathrm{n}-\mathrm{OH}$, the repeating unit $\mathrm{C}_{5} \mathrm{H}_{8}$ is called isoprene. The successive condensation of $\mathrm{C}_{5} \mathrm{H}_{8}$ units forms simple isoprenoids. In Figure 1:1 is shown an example of $2 \mathrm{E}$-geraniol, which has $\mathrm{n}=2$. Isoprenoids are biosynthesized from the branched five-carbon isopentenyl diphosphate (IPP) and its isomer dimethylallyl diphosphate (DMAPP) ${ }^{17}$. Carotenoids that have a polyisoprenoid structure (not shown here) have an essential role as anti-oxidants and are precursors of vitamin $A^{18}$. 
Saccharolipids have fatty acid chains attached to the sugar-based backbone. Figure 1:1 shows a structure of Kdo2-lipid A which is an essential structural component of all Gram-negative bacteria as it makes them more viable ${ }^{19}$.

Many frequently used antimicrobial, antiparasitic, and even anticancer medicating agents are polyketides and their derivatives ${ }^{8}$. One prominent example of these drugs includes erythromycins (first isolated from bacteria), shown in Figure 1:1 (erythromycin C). Polyketides are structurally diverse compounds that are found in microorganisms, animals, and plants. These compounds often include cyclic backbones, the diversity comes from, but not limited to, the addition of $-\mathrm{OH},-\mathrm{CH}_{3}$ or sugary (glycan) groups through various biosyntheses ${ }^{20}$.

Sphingomyelins of sphingolipids and glycerophospholipids have quite similar structures as could be noted in Figure 1:2. These compounds contain a glycerol backbone, except for sphingomyelin. More importantly, these compounds contain phosphate groups with lipid class-specific headgroups. There are six main glycerophospholipids: phosphatidylethanolamine (PE), phosphatidylcholine (PC), phosphatidylserine (PS), phosphatidylinositol (PI), phosphatidylglycerol (PG), and phosphatidic acid (PA). Sphingomyelins are part of the sphingolipid group as they contain an 18-carbon amino alcohol (sphingosine) group. However, they also have a phosphate group with either choline or ethanolamine headgroup in their structure. Sphingomyelins with an ethanolamine headgroup (ceramide phosphoethanolamine) are found in insects and invertebrates, while sphingomyelins with a choline headgroup (ceramide phosphocholine) are part of mammal tissues ${ }^{8,21}$. Sphingomyelins and glycerophospholipids together are called as phospholipids. 
Glycerophospholipids have a phosphate group in an sn-3 location on the glycerol backbone. Lysoglycerophospholipids have one fatty acyl esterified at the sn-1 position and a phosphate group at the sn-3 position of the glycerol, while glycerophospholipids often have two different fatty acyls esterified at the $s n-1$ and the $s n-2$ positions and a phosphate group at the sn-3 position of the glycerol.

There are different kinds of nomenclature that can be used for phospholipids ${ }^{22}$, but short notations are frequently used in scientific literature. For example, $\mathrm{PC}(17: 0 / 22: 4)$ describes a phosphatidylcholine with an $s n-1 \mathrm{R}$ group having 17 carbons and 0 double bonds and an sn-2 R group having 22 carbons and 4 double bonds. Alternatively, it is common to express $\mathrm{PC}(17: 0 / 22: 4)$ as $\mathrm{PC}(39: 4)$ to show the sum of carbon atoms and double bonds as identifying the individual fatty acids requires specialized analytical methodologies not available in every laboratory.

There are also more complex glycerophospholipids (structures not shown). For glycerophosphoglycerols and glycerophosphoglycerophosphates there is a second glycerol unit that is part of the head group (for example, which would be instead of choline head group in Figure 1:2). There are also glycerophosphoglycerophosphoglycerols or simply called cardiolipins; these structures as the name implies have three glycerol units (a third glycerol unit is usually acylated at the $s n-1$ ' and $s n-2$ ' positions). Cardiolipins are pseudosymmetrical molecules and are part of mitochondrial lipids ${ }^{23,24}$. Lastly, the smallest glycerophospholipids are phosphatidic acid molecules (structures not shown); these lipids are like the rest of the glycerophospholipids except they do not have head group after 
phosphate group. Phosphatidic acid molecules are primary precursors of many other lipids that form via biosythesis ${ }^{25}$.

Phospholipids are amphiphilic as they have a polar head group and non-polar fatty acyl chains. In cell membranes, phospholipid structure allows the formation of the bilayers of the membranes. A cell membrane diagram is shown in Figure 1:3; it consists of a bilayer and proteins embedded into it (proteins are in blue on the diagram). Cell membranes define cellular boundaries and are dynamic structures which are permeable, elastic, and fluid ${ }^{26}$.

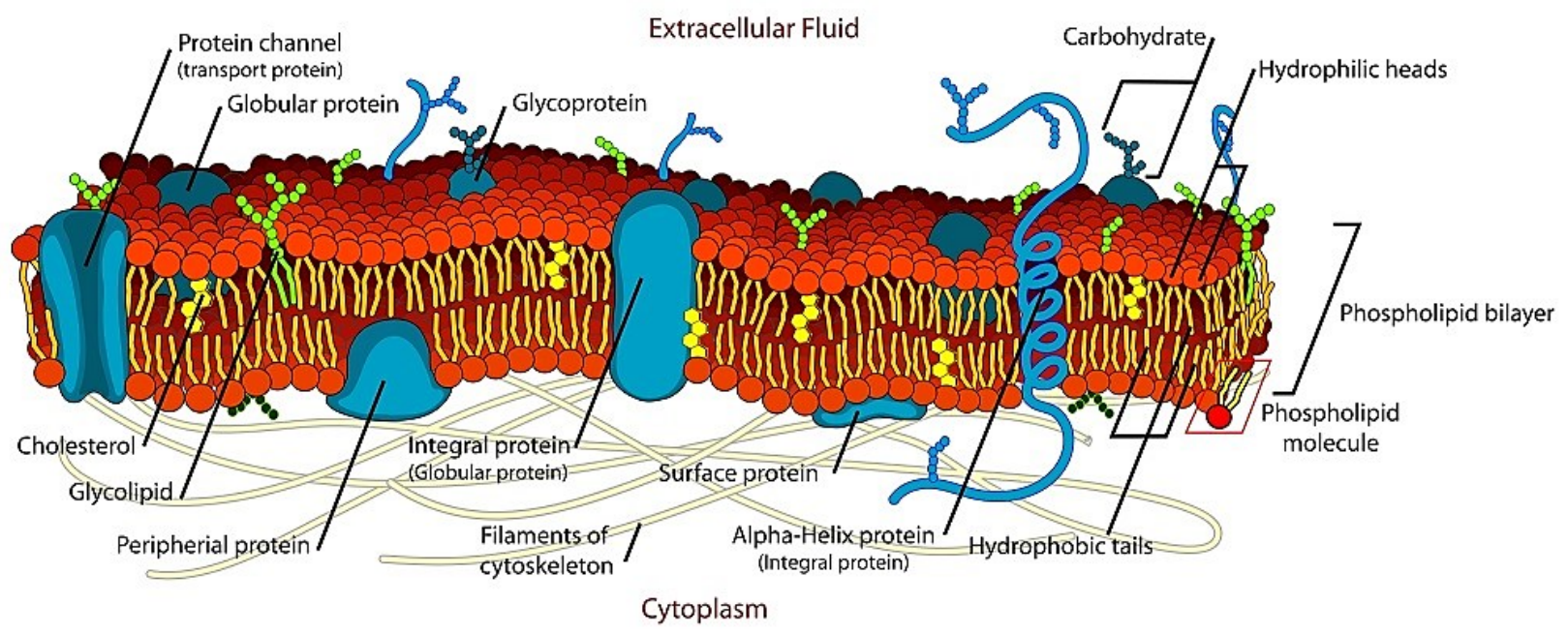

Figure 1:3. Cell membrane diagram. This Figure was adapted from https://biologydictionary.net/cellmembrane/

Phospholipids are the main constituents of the bilayer of cellular membranes 8,27 . The molecular composition of a given bilayer varies with cell and tissue type in an organism dependent manner ${ }^{14,28}$. It has been observed that PCs and PEs are more abundant structural components of membranes than other phospholipids, this pattern has been seen in plants ${ }^{29}$, goldfish ${ }^{30}$, frogs ${ }^{14}$, and mammals ${ }^{31,32}$. It has also been observed 
that $\mathrm{PI}$ concentrations in membranes are the smallest compared to other glycerophospholipids ${ }^{14,30}$. In mammalian cells, the outer layer of the plasma membrane contains PC and SM lipids predominantly, whereas PS, PE, and PI lipids are found in the inner layer of the bilayer ${ }^{33}$.

Some examples of lipid classes have been been shown and discussed above. However, there are quite a few subclasses of the classes of lipids. In mammalian cell membranes, there are more than 1000 major lipid species ${ }^{27}$. Lipidomics is still relatively new field compared to other "omics" fields (since 2003). However, it has been growing rapidly and steadily ${ }^{34}$. Due to structural diversity and complexity of multiple lipid species, it is almost impossible to find one analytical technique to analyze them all, which presents many challenges. Furthermore, novel mass spectrometry-based technologies are emerging to achieve complete lipidome identification, comprehensive structural characterization, and precise quantitation for all individual lipid species that may be present within a biological sample of interest.

\subsection{Animals adaption to cold}

Different winter coping mechanisms exist that help certain species to survive cold climates and overwinter. Winter conditions can be very harsh which make a survival into a quite challenging event. Some examples of harsh conditions include: air temperatures are low, available water freezes, food, and shelter are hard to find, and the available daylight is less compared to summer months.

Migration is one example of cold adaptation. Migration is an animal behavior where all the animals in the population move to a warmer climate to avoid the cold weather of 
winter. This behavior is typical among birds that live in the northern countries such as Canada geese, warblers, red-headed woodpeckers and ducks (Figure 1:4).
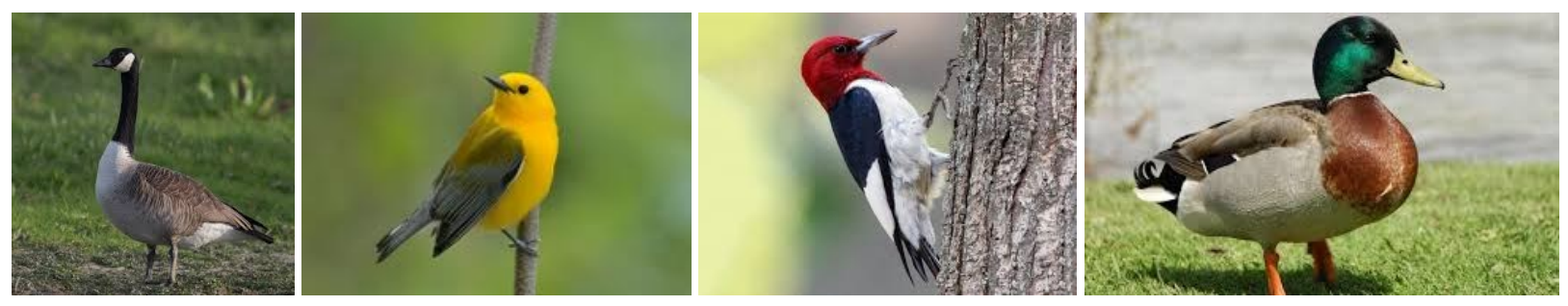

Figure 1:4. Examples of birds that migrate to warmer climates to avoid cold winter. Examples on the photos are from left to right: Canada goose, warbler, red-headed woodpecker and duck.

Hibernation is another cold coping mechanism; it is due to mostly low food availability. Hibernation is where animals become dormant or inactive during the coldest months. Hibernating animals use considerably less energy during cold months to survive, and they sleep in hidden places to keep safe. By hibernating, animals can conserve energy over the entire winter by having low metabolic activity, low oxygen use, lower heart rate and low body heat. Hibernating animals need to prepare themselves for overwintering by eating more to build up fat (white adipose tissue (WAT)), and some animals also hide food for winter that they can consume when they wake up for short periods of times during winter (called arousal periods) $)^{35}$. Same examples of hibernators include bats, bears, mice and ground squirrels.

Many amphibians have another way tolerating cold as they cannot maintain steady body temperature; they have cold tolerance mechanisms. They spend winters buried in the mud at the bottom of ponds and wetlands where they become dormant for long periods of time. These amphibians have increased levels of glucose in their blood which 
acts as natural antifreeze. This antifreeze protects their organs by lowering the temperature at which their body freezes. They also have nucleating proteins that regulate extracellular freezing. For these animals, up to $65 \%$ of their bodies may freeze ${ }^{36}$. Because of this adaptation, many north-eastern frogs can withstand temperatures below freezing without harm (e.g., cell-volume reduction, protein denaturation, structural damage) ${ }^{37}$. Some examples of freeze tolerant animals include frogs, turtles, snails, and insects.

Freeze avoidance is another example of cold survival mechanisms. This mechanism happens through supercooling. Supercooling allows for bodily fluids to remain liquid below the temperature of the freezing/melting point. Supercooling is possible due to a lack of ice nucleators; this is controlled by antifreeze proteins and cryoprotectants (most often glycerol $)^{38}$. This survival strategy is used by many insects.

Both freeze avoidance and freeze tolerance are powerful cold-coping strategies; however, it is not entirely understood why a particular species adopt one over the other. It has been observed that freeze tolerance is particularly advantageous in habitats where winter temperatures are relatively mild and unpredictable ${ }^{39}$. Supercooling, on the other hand, permits the survival over a broader temperature range. However, freezing can occur suddenly at any temperature below the equilibrium of freezing/melting point, which is lethal to the animal ${ }^{37}$. Some characteristics of freeze tolerance and freeze avoidance are summarized in Figure 1:5.

Other animals change their physical characteristics to help them to cope with winter. Some examples include growing more and thicker fur (e.g., muskox), changing fur color (e.g., rabbit), blubber storage for heat and energy (seal), huddling to stay close to each other to keep warmth (e.g., penguin). It is of importance to note that captive animals 
often lose the need for hibernation or other cold coping mechanisms. However, some circadian or circannual rhythms are maintained even in captive animals ${ }^{40}$.

Supercooling

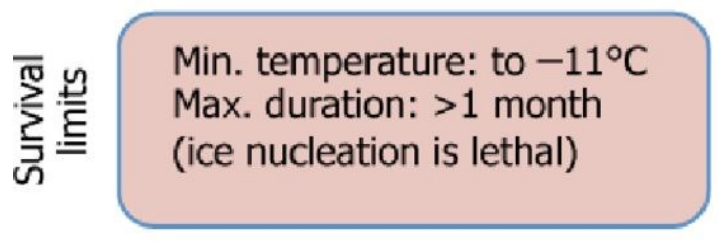

Freeze Tolerance

Min. temperature: -4 to $-6^{\circ} \mathrm{C}$

Max. duration: several weeks

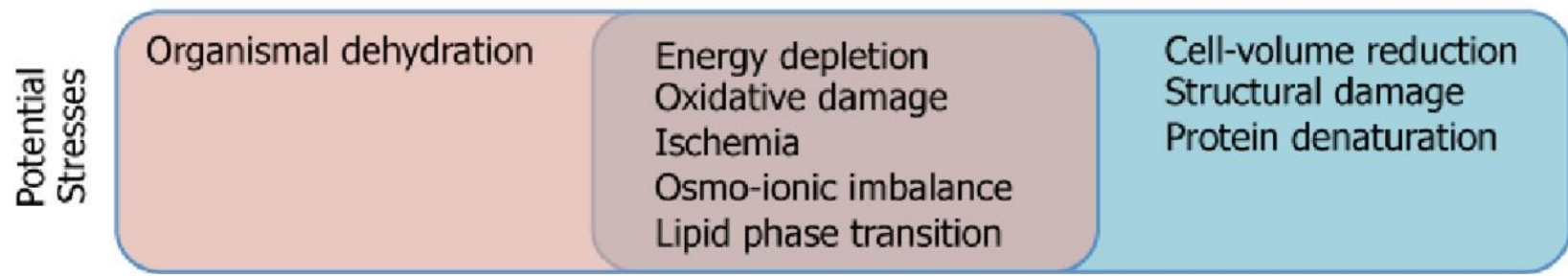

Figure 1:5. Characteristics of freeze avoidance (through supercooling) and freeze avoidance. This Figure was adapted from Costanzo and Lee, $2013^{37}$.

The details of each specific coping mechanism for particular species will be given in the chapters to follow. It is important to note that active phase hibernating animals have approximately the same physiological profile as non-hibernating species. However, hibernating species can change their phenotype to adapt to new physiological states; this would be deadly for non-hibernators. The mechanisms of metabolic depression, gain and loss of body weight regulation, the extended fasting, as well as extended hypothermia while resisting deleterious effects and other characteristics of hibernation could translate to using these mechanisms in the medical field if better understood ${ }^{41}$. Hibernation would mean that organs need less oxygen to function, breathing would slow down, and heart would not need to pump as fast. Hypothetically, the injured person would lose less blood if they would be placed into a hibernation-like state. Also, that could translate to organs, 
which usually do not get enough oxygen because of bleeding, needing less oxygen and thus causing them to suffer less damage. $\mathrm{H}_{2} \mathrm{~S}$ gas has been shown to put mice into a suspended animation-like state which made them survive for hours in low oxygen environments that would typically kill them ${ }^{42,43}$.

\subsection{Cell membrane adaptation to cold}

As animals adapt to cold, so do their cell membranes. Membranes adjust to cold temperatures by adjusting their fluidity; this is to prevent rigidifying of membranes as the environmental temperatures decrease ${ }^{44,45}$. It is like coconut oil (melting point $24^{\circ} \mathrm{C}$ ), a saturated triglyceride, which is fluid at higher room temperatures but solid at lower temperatures. Unsaturated soybean oil (melting point $-16^{\circ} \mathrm{C}$ ) would stay liquid in a refrigerator compared to coconut oil. Both of these oil structures are shown in Figure 1:6.

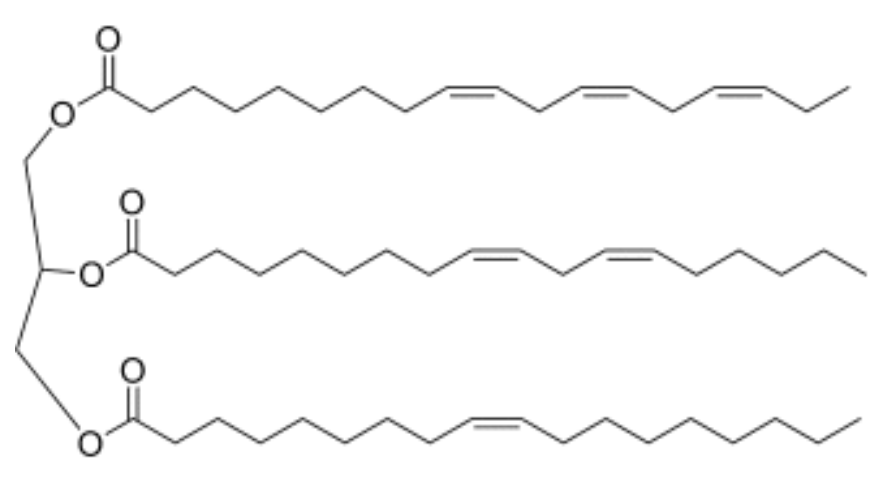

Soybean Oil

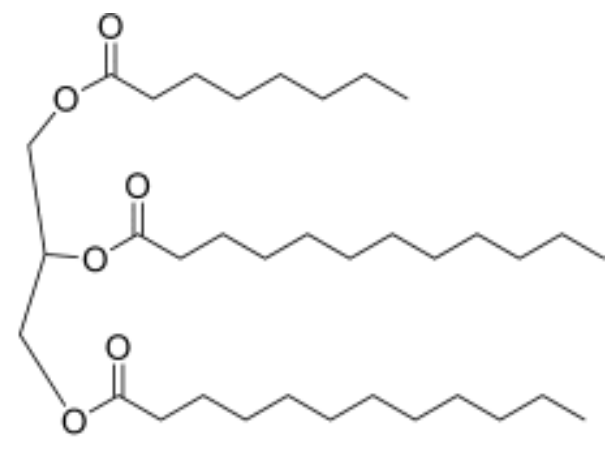

Coconut Oil

Figure 1:6. Molecular structures of soybean and coconut oils. This Figure was adapted from Costa et al., $2016^{46}$. 
To be able to adjust to cold temperature conditions and maintain optimal membrane fluidity, cells regulate the expression of lipid desaturases ${ }^{44,47}$. Weber and Marahiel, 2003 have shown for B. subtilis (Gram-positive bacteria) that histidine kinases (membrane-bound enzymes) can sense the loss of fluidity and act to change fluidity to ensure homeoviscous adaption and continued function at low temperatures ${ }^{48}$. Membranes are more fluid as the degree of unsaturation or the environment temperature increases or the aliphatic chain length decreases ${ }^{49}$. In adjustment to cold temperatures, shorter lipid chains and branched-chain lipids are produced via biosynthesis shown in various bacterial research ${ }^{50-52}$. The visual representation of membrane fluidity with unsaturated versus saturated fatty acid chains of phospholipids is shown in Figure 1:7.
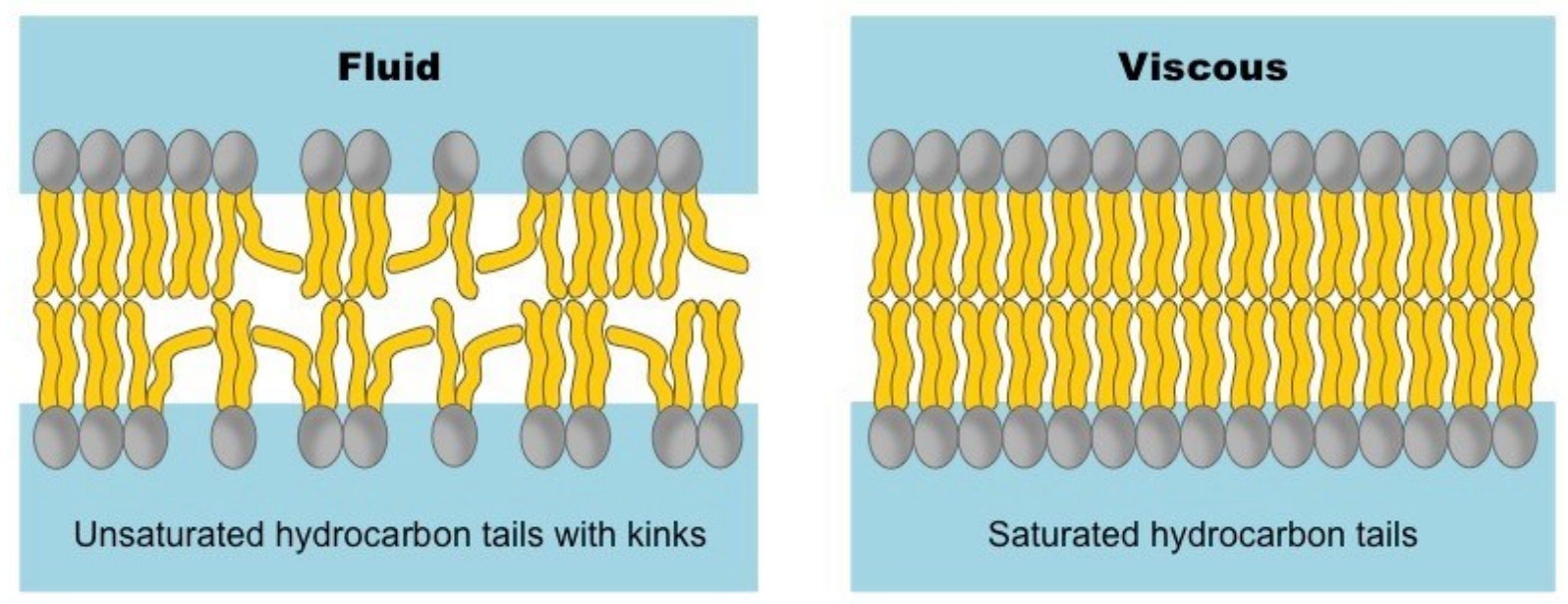

Figure 1:7. Visual representation of two kinds of the lipid bilayers. On the right, phospholipid fatty acyl chains are saturated (no double bonds) making the tails tightly packed making bilayer more viscous; on the left, phospholipid fatty acyl chains are unsaturated (has double bonds) making the tails have kinks making the bilayer more fluid. This Figure was adapted from http://ib.bioninja.com.au/standardlevel/topic-1-cell-biology/13-membrane-structure/membrane-fluidity.html

PC, PE and SM species account for up to $80 \%$ of all glycerophospholipids in most biological membranes ${ }^{53,54}$. Because of this, PC/PE and PC/SM ratios have been used to 
describe membrane fluidity ${ }^{28,54,55}$. The geometry of PC and PE head groups is different; PE has a conical shape and PC has a cylindrical shape (Figure 1:8). The conical shape of PE prevents the close packing of lipids, allowing for more molecular movement within the membrane and, therefore, the increased fluidity of the membranes ${ }^{14}$. In bacterial research, it has been observed that expression of phosphatidylserine decarboxylase (which produces PE lipids in some gram-negative bacteria) increases in response to cold ${ }^{51,56}$. Also, even though, PC and SM have the same choline head group, it has been suggested that PC is a more hydrated than SM, which would allow SM to pack tighter than $\mathrm{PC}^{57}$. Thus, in a more fluid membrane, there would be smaller PC/PE ratios and higher PC/SM ratios compared to a more rigid membrane.

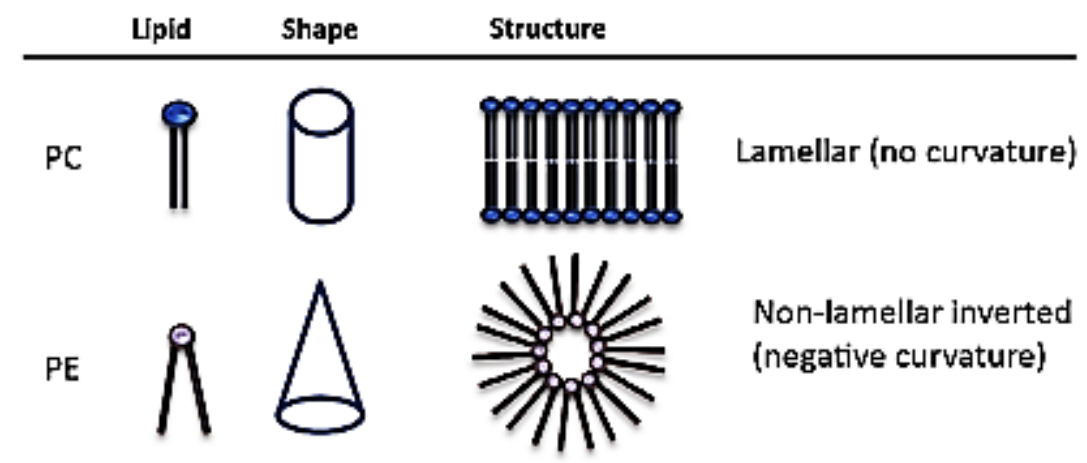

Figure 1:8. Shape and structure of phosphatidylcholine (PC) and phosphatidylethanolamine (PE). This Figure was adapted from Zalba et al., $2017^{58}$.

\subsection{The goals of the thesis}

The focus of this dissertation is mainly lipidomic work, specifically interest is to look at relative quantitative changes of phospholipids in different cold adaption mechanisms. There are three animal models chosen for this research work. Thirteen-lined ground 
squirrel for hibernation adoption is investigating phospholipid differences in liver tissue between summer and winter months and is the focus of Chapter 3. Wood frog for freeze tolerance adoption is investigating phospholipid differences in liver and leg muscle tissues between summer and winter months and is the focus of Chapter 4 . It is of interest to look at vital versus non-vital tissues and observe the differences in phospholipid behaviour in these two tissues in cold adaption. Chapter 5 is looking at phospholipid differences in goldfish eye retina tissue between January and July samples as it has been observed that goldfish eye retina lipid bilayer has a tendency to be more rigid during summer months. These three chapters investigate lipid membrane fluidity in different animal models and tissues as the response to cold stress. In Chapter 5 will be shown goldfish eye retina fluidity changes in the absence of the environmental cues and discussed will be preserved semiannual behaviour response of goldfish eye retina lipid bilayer.

Chapter 2 is an instrumental introductory chapter which gives an overview of different mass spectrometers used for various research work in this thesis. Chapter 6 focuses on the phospholipid data analysis after the data collection to highlight all the necessary steps in the data processing done in Chapters $3-5$. Chapter 7 is an independent chapter and focuses on proteomics research that was done as a side project from the main phospholipid research. The focus in Chapter 7 is to look and Trimethylation Enhancement using Diazomethane ( $\mathrm{TrEnDi})$ technique in peptide and protein research; it will demonstrate some success and challenges faced with this technique. 


\subsection{References}

(1) Fahy, E.; Subramaniam, S.; Murphy, R. C.; Nishijima, M.; Raetz, C. R. H.; Shimizu, T.; Spener, F.; van Meer, G.; Wakelam, M. J. O.; Dennis, E. A. J. Lipid Res. 2009, 50 Suppl (Suppl), S9-14.

(2) Subramaniam, S.; Fahy, E.; Gupta, S.; Sud, M.; Byrnes, R. W.; Cotter, D.; Dinasarapu, A. R.; Maurya, M. R. Chem. Rev. 2011, 111 (10), 6452-6490.

(3) van Eyk, H. J.; van Schinkel, L. D.; Kantae, V.; Dronkers, C. E. A.; Westenberg, J. J. M.; de Roos, A.; Lamb, H. J.; Jukema, J. W.; Harms, A. C.; Hankemeier, T.; van der Stelt, M.; Jazet, I. M.; Rensen, P. C. N.; Smit, J. W. A. Nutr. Diabetes 2018, 8 (1), 6.

(4) Grundy, S. M.; Denke, M. A. J. Lipid Res. 1990, 31 (7), 1149-1172.

(5) Mayberg, M. R.; Batjer, H. H.; Dacey, R.; Diringer, M.; Haley, E. C.; Heros, R. C.; Sternau, L. L.; Torner, J.; Adams, H. P.; Feinberg, W. Stroke 1994, 25 (11), 23152328.

(6) Kulkarni, H.; Meikle, P. J.; Mamtani, M.; Weir, J. M.; Barlow, C. K.; Jowett, J. B.; Bellis, C.; Dyer, T. D.; Johnson, M. P.; Rainwater, D. L.; Almasy, L.; Mahaney, M. C.; Comuzzie, A. G.; Blangero, J.; Curran, J. E. Hypertens. (Dallas, Tex. 1979) 2013, 62 (3), 621-626.

(7) Panahi, Y.; Ahmadi, Y.; Teymouri, M.; Johnston, T. P.; Sahebkar, A. J. Cell. Physiol. 2018, 233 (1), 141-152.

(8) Fahy, E.; Subramaniam, S.; Brown, H. A.; Glass, C. K.; Merrill, A. H.; Murphy, R. C.; Raetz, C. R. H.; Russell, D. W.; Seyama, Y.; Shaw, W.; Shimizu, T.; Spener, F.; van Meer, G.; VanNieuwenhze, M. S.; White, S. H.; Witztum, J. L.; Dennis, E. A. J. Lipid Res. 2005, 46 (5), 839-861.

(9) Hunter, J. E. Lipids 2006, 41 (11), 967-992.

(10) Prentki, M.; Madiraju, S. R. M. Endocr. Rev. 2008, 29 (6), 647-676.

(11) Cuculescu, M.; Hyde, D.; Bowler, K. J. Therm. Biol. 1995, 20 (1/2), 207-222.

(12) Crockett, E. L. Amer. Zool. 1998, 38, 291-304.

(13) Scapin, S.; Baldini, P.; Luly, P. Lipids 1990, 25 (8), 443-449.

(14) Reynolds, A. M.; Lee, R. E.; Costanzo, J. P. J Comp Physiol B 2014, 184, 371383.

(15) Park, H.; Go, Y. M.; St John, P. L.; Maland, M. C.; Lisanti, M. P.; Abrahamson, D. R.; Jo, H. J. Biol. Chem. 1998, 273 (48), 32304-32311.

(16) Incardona, J. P.; Eaton, S. Curr. Opin. Cell Biol. 2000, 12 (2), 193-203.

(17) Lichtenthaler, H. K.; Schwender, J.; Disch, A.; Rohmer, M. FEBS Lett. 1997, 400 (3), 271-274. 
(18) Rao, A. V.; Rao, L. G. Pharmacol. Res. 2007, 55 (3), 207-216.

(19) Wang, X.; Quinn, P. J.; Yan, A. Biol. Rev 2015, 90, 408-427.

(20) Hernandez, E.; Kamal-Eldin, A. Processing and nutrition of fats and oils; Institute of Food Technologists Series; Wiley: Houston, Texas, 2013.

(21) Vacaru, A. M.; Van Den Dikkenberg, J.; Ternes, P.; Holthuis, J. C. M. J. Biol. Chem. 2013, 288 (16), 11520-11530.

(22) J. Lipid Res. 1978, 19 (1), 114-128.

(23) Chang, Y.-Y.; Kennedy, E. P. J. Lipid Reseach 1967, 8, 447-455.

(24) Schlame ', M.; Brody, S.; Hostetler', K. Y. Eur. J. Biochem 1993, 212, 727-735.

(25) Athenstaedt, K.; Daum, G. Eur. J. Biochem. 1999, 266 (1), 1-16.

(26) Rawicz, W.; Smith, B. A.; McIntosh, T. J.; Simon, S. A.; Evans, E. Biophys. J. 2008, 94 (12), 4725-4736.

(27) van Meer, G. EMBO J. 2005, 24 (18), 3159-3165.

(28) Fajardo, V. A.; McMeekin, L.; LeBlanc, P. J. J. Membr. Biol. 2011, 244 (2), 97103.

(29) Furt, F.; Simon-Plas, F.; Mongrand, S. In The Plant Plasma Membrane; Murphy, A. S., Peer, W., Schulz, B., Eds.; Springer Verlag, 2011; pp 3-30.

(30) Fliesler, S. J.; Maude, M. B.; Anderson, R. E. Biochim. Biophys. A cta 1983, 734, 144-152.

(31) Vance, J. E. Traffic 2015, 16 (1), 1-18.

(32) Aloia, R. C. Comp. Biochem. Physiol. 1981, 68B, 203-208.

(33) Watson, H. Essays Biochem. 2015, 59, 43-69.

(34) Rustam, Y. H.; Reid, G. E. Anal. Chem. 2018, 90, 374-397.

(35) Nelson, C. J.; Otis, J. P.; Carey, H. V. J. Physiol. 2009, 587 (9), 1863-1870.

(36) Storey, K. B.; Storey, J. M. Annu. Rev. Physiol 1992, 54, 619-637.

(37) Costanzo, J. P.; Lee, R. E. J. Exp. Biol. 2013, 216 (Pt 11), 1961-1967.

(38) Storey, K. B.; Storey, J. M. Physiol. Rev. 1988, 68 (1).

(39) Sinclair, B. J.; Addo-Bediako, A.; Chown, S. L. Biol. Rev. Camb. Philos. Soc. 2003, 78 (2), 181-195.

(40) Jansen, H. T.; Leise, T.; Stenhouse, G.; Pigeon, K.; Kasworm, W.; Teisberg, J.; Radandt, T.; Dallmann, R.; Brown, S.; Robbins, C. T. Front. Zool. 2016, 13, 42.

(41) Carey, H. V; Andrews, M. T.; Martin, S. L. Physiol Rev 2003, 83, 1153-1181.

(42) Asfar, P.; Calzia, E.; Radermacher, P. Crit. Care 2014, 18 (215). 
(43) Blackstone, E.; Morrison, M.; Roth, M. B. Brevia. (Rome). 2005, 308, 518-519.

(44) Los, D. A.; Murata, N. Biochim Biophys Acta. 2004, 1666 (1-2), 142-157.

(45) Nichols, D. S.; Miller, M. R.; Davies, N. W.; Goodchild, A.; Raftery, M.; Cavicchioli, R. J. Bacteriol. 2004, 186 (24), 8508-8515.

(46) Costa, C. S. M. F.; Fonseca, A. C.; Moniz, J.; Godinho, M.; Serra, A. C.; Coelho, J. F. J. Ind. Crops Prod. 2016, 85, 403-411.

(47) Cybulski, L. E.; del Solar, G.; Craig, P. O.; Espinosa, M.; de Mendoza, D. J. Biol. Chem. 2004, 279 (38), 39340-39347.

(48) Weber, M. H.; Marahiel, M. A. Sci. Prog. 2003, 86 (Pt 1-2), 9-75.

(49) Fan, W.; Evans, R. M. Cell 2015, 161, 962-963.

(50) Rodrigues, D. F.; Ivanova, N.; He, Z.; Huebner, M.; Zhou, J.; Tiedje, J. M. BMC Genomics 2008, 9, 547.

(51) Bergholz, P. W.; Bakermans, C.; Tiedje, J. M. J. Bacteriol. 2009, 191 (7), 2340 2352.

(52) Ponder, M. A.; Thomashow, M. F.; Tiedje, J. M. Extremophiles 2008, 12 (4), 481490.

(53) Escribá, P. V; González-Ros, J. M.; Goñi, F. M.; Kinnunen, P. K. J.; Vigh, L.; Sánchez-Magraner, L.; Fernández, A. M.; Busquets, X.; Horváth, I.; BarcelóCoblijn, G. J. Cell. Mol. Med. 2008, 12 (3), 829-875.

(54) Borochov, H.; Zahler, P.; Wilbrandt, W.; Shinitzky, M. Biochim. Biophys. Acta Biomembr. 1977, 470 (3), 382-388.

(55) Owen, J. S.; Bruckdorfer, K. R.; Day, R. C.; McIntyre, N. J. Lipid Res. 1982, 23 (1), 124-132.

(56) Chintalapati, S.; Kiran, M. D.; Shivaji, S. Cell. Mol. Biol. (Noisy-le-grand). 2004, 50 (5), 631-642.

(57) M'Baye, G.; Mély, Y.; Duportail, G.; Klymchenko, A. S. Biophys. J. 2008, 95 (3), $1217-1225$.

(58) Zalba, S.; ten Hagen, T. L. M. Cancer Treat. Rev. 2017, 52, 48-57. 


\section{Chapter 2. Mass spectrometry instruments and high-performance liquid chromatography separation}

\subsection{Mass spectrometry}

\subsubsection{The mass spectrum}

Mass spectrometry (MS) is an instrumental analytical technique that measures the mass-to-charge $(\mathrm{m} / \mathrm{z})$ ratio of an element or compound of interest. The first task is to convert analyte molecules in the sample into ions; this may be done by protonating the molecules in the gas phase. The ions are then separated according to their mass-tocharge ratios in an electric or magnetic field ${ }^{1}$. Finally, the ions of a mass-to-charge ratio are collected on a detector. The signal on the detector is amplified and sent to a recorder which is shown as a mass spectrum - a plot of relative intensity versus $\mathrm{m} / \mathrm{z}$. An example of a mass spectrum of protonated caffeine is shown in Figure 2:1.

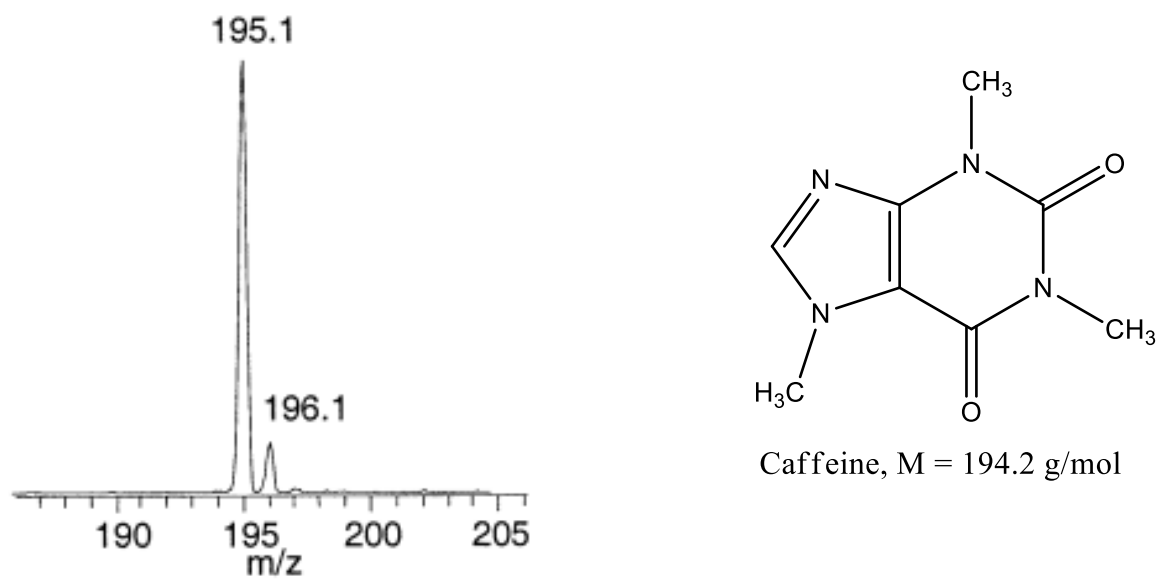

Figure 2:1. ESI mass spectrum of protonated caffeine. This Figure was adapted from Schwartz et al., $2002^{2}$. 


\subsubsection{Electrospray ionization}

Before compounds can be sent to the mass spectrometer for analysis, they must pass through an ionization source. The ionization technique employed in this thesis work was electrospray ionization (ESI). ESI uses solvents containing the analytes of interest and sprays them into the MS instrument through a capillary connected to a high voltage. As the analytes emerge out of the solution phase and into the gas phase, they are protonated to form gas phase ions. Formation of negatively charged ions is also possible, however, mostly protonated species were analyzed in this thesis work and focused on. ESI is amenable to high-performance liquid chromatography (HPLC) as separated compounds in the sample flow directly from the separating HPLC column to the ESI capillary. The variations to the ESI technique come from the size of the spraying capillary tip, as different flow rates can be achieved with different tips. The conventional ESI tip has flow rates of $4-200 \mu \mathrm{L} / \mathrm{min}^{3}$, while nanospray tips have flow rates of $20-50 \mathrm{~nL} / \mathrm{min}^{4}$. Nanospray ionization consumes less sample during the analysis which means less sample is needed for the experiment and the sample lasts longer in the capillary, which also enables multiple experiments to be performed ${ }^{4}$. Nanospray also has the advantage of being more tolerant towards salt contamination and higher water content in the solvent (usually lower water content is preferred for simpler solvent evaporation) $)^{5,6}$.

The mechanism of ESI is shown in Figure 2:2. There are four steps for ESI: formation of ions (this is usually done before the spray with HPLC solvents), nebulization (aerosol generation), desolvation and ion evaporation. In nebulization step, solvent and high-flow gas are combined in the spraying needle at a strong voltage (2-6 kV); this breaks sample solution into droplets. These droplets disperse and because of electrostatic forces 
in the chamber ions of the same polarity are collected at the droplet surface. These droplets are then evaporated of solvent reducing the droplet size and concentrating the ions. At about $10^{8} \mathrm{~V} / \mathrm{cm}^{3}$ of charge density, ion evaporation from the droplet occurs. These ions are then attracted to enter and pass through a capillary into the ion optics and the mass analyzer.

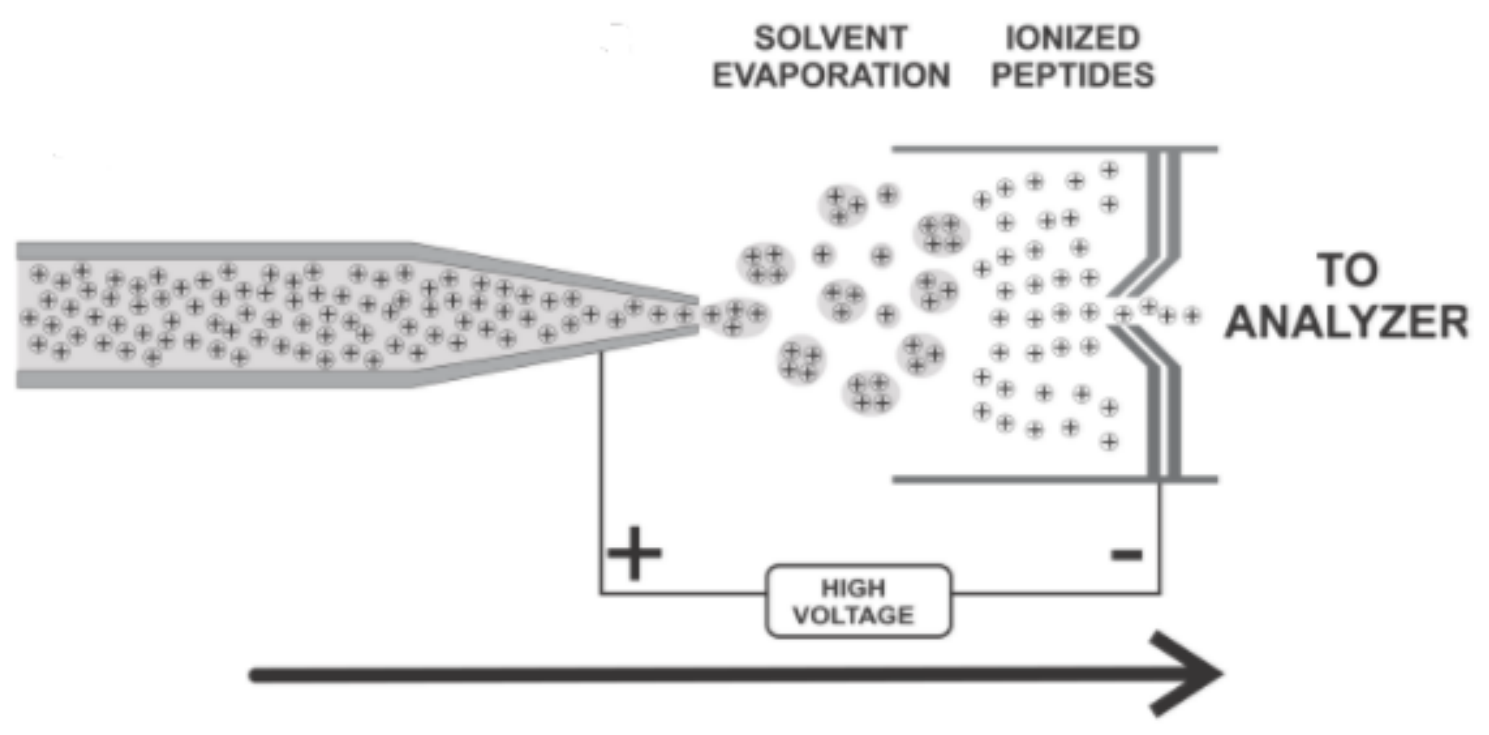

Figure 2:2. Mechanism of electrospray ionization. This Figure was adapted from Demartini, $2013^{7}$.

\subsubsection{Quadrupole}

Figure 2:3 shows the basic design of the quadrupole analyzer. A quadrupole consists of 4 parallel metal rods. Opposing poles are charged with a direct current (DC) voltage and an alternating current $(\mathrm{AC})$ at a controllable radio frequency (RF) $)^{8}$. Two opposite poles have a positive charge, and the other two poles have a negative charge; these polarities change during the experiment. The AC and DC voltages are tuned so that only ions with a specific mass-to-charge $(\mathrm{m} / \mathrm{z})$ ratio will oscillate along the field to eventually reach the detector. Other non-resonant ions will be deflected off the path and 
will not be detected. The voltages are rapidly changed on the quadrupole so that ions of mass after another take their turns on the stable oscillating path and are collected on the detector $^{9}$. All the voltage changes are done automatically by the instrument, just scan range needs to be selected by the MS user.

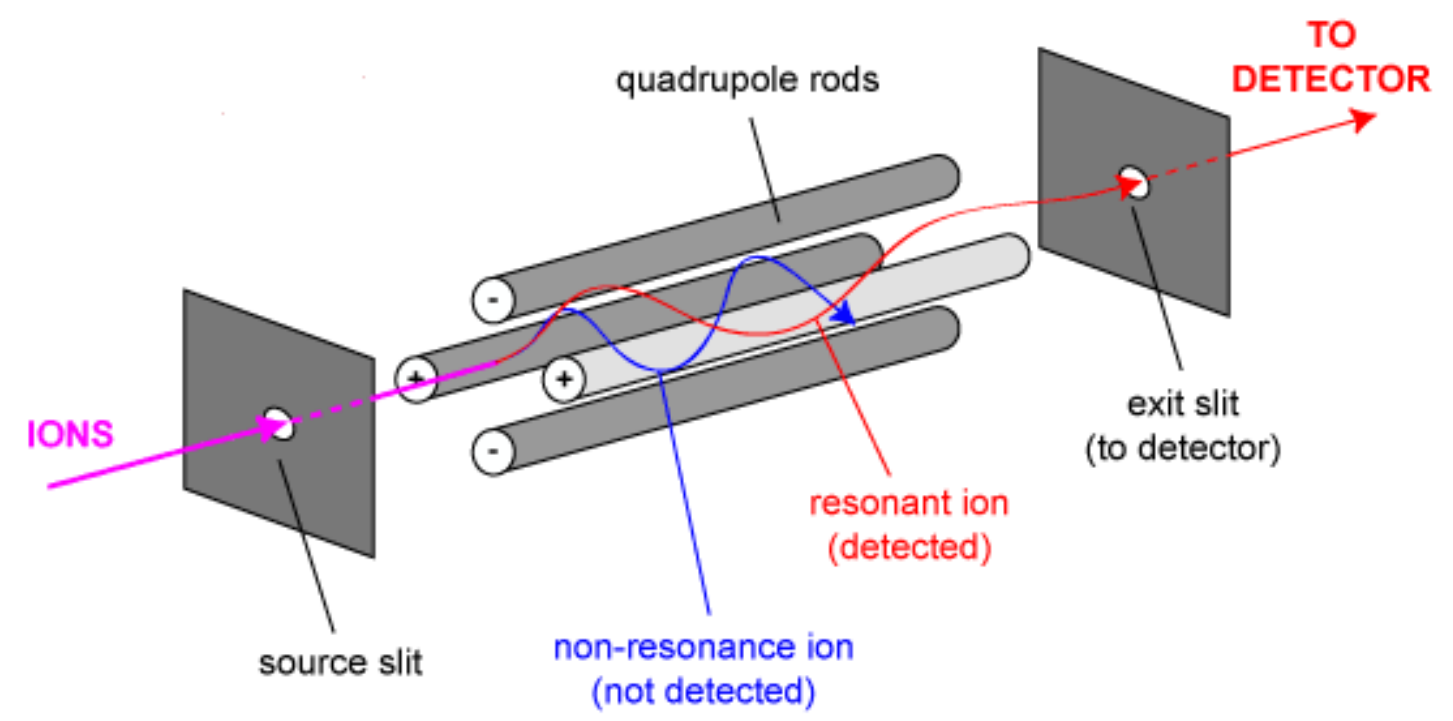

Figure 2:3. Schematic of a quadrupole analyzer. This Figure was adapted from http://www.bris.ac.uk/nerclsmsf/techniques/gcms.html

Quadrupole MS instruments do not depend on the kinetic energy of the ions generated and have high transmission rates $^{8}$. Also, these instruments have a high scanning rate: Christian et al., 2014 reports that quadrupole MS can scan 20 spectra per second over a range of 800 mass units ${ }^{8}$. However, quadrupole MS can only detect up to $4000 \mathrm{~m} / \mathrm{z}$ (time of flight instruments, on the other hand, have no limit for mass detection) and has a maximum resolution of $5,000^{10,11}$. Resolution is defined as $\mathrm{m} / \Delta \mathrm{m}$; for example, for masses 100.00 and 100.02 , the resolution of the instrument is $100.00 / 0.02$, i.e. 5,000 . 


\subsubsection{Time of flight}

The generated ions from the ionization source are accelerated into the time of flight (TOF) tube using repeller or accelerator plates (these could supply 1-10 kV of energy) ${ }^{12}$. This way every ion is supplied with constant kinetic energy for the flight ${ }^{13}$. In the TOF instruments, the lighter ions will fly faster than heavier ones and thus reach the detector faster. The longer the TOF tube, the longer time ions will spend flying in the tube, and the longer time they will have to separate from each other. Better resolution is obtained with longer tubes. To make TOF tubes longer without taking too much physical linear space TOF instruments come with a reflectron. This reflectron uses electric fields to change the direction of the traveling ions, sending them back in the same direction they came from. Different reflectrons have been developed; some have homogeneous while others inhomogeneous (better transmittance across broader $\mathrm{m} / \mathrm{z}$ range) electric fields ${ }^{11}$. Smaller ions penetrate less deeply into the charged field than larger ions. The deeper an ion penetrates into the field of the reflectron, the larger repulsive energy it will need on its way out towards detector ${ }^{11}$. Improvements in mass resolution are achieved because ions of different kinetic energies penetrate the reflectron to differing degrees (this reduces spread in kinetic energy of the ions with the same $\mathrm{m} / \mathrm{z}$ correcting for axial displacement). A schematic of reflectron TOF-MS is shown in Figure 2:4.

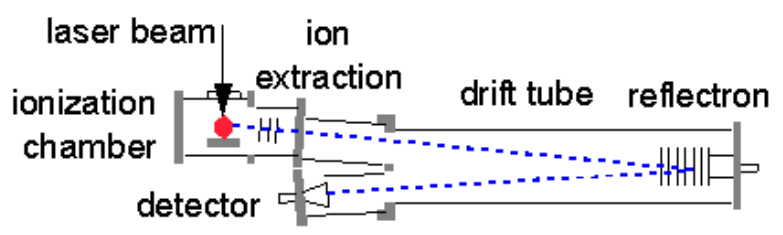

Figure 2:4. Schematic of reflectron TOF-MS. This Figure was adapted from http://elchem.kaist.ac.kr/vt/chem-ed/ms/tof.htm 
More common are orthogonal acceleration TOF-MS instruments; these have TOF tube perpendicular to the direction of the ions initially generated. With this design generated ions can be controlled and accelerated more uniformly than in a simple reflectron TOF-MS, which equips orthogonal TOF-MS instruments with even higher resolution power ${ }^{14,15}$. In orthogonal TOF-MS instruments ions of the same $\mathrm{m} / \mathrm{z}$ hit the detector at the same time.

The resolving power of a linear TOF MS instrument is low as not all ions will get same acceleration potential which means spread of kinetic energy and velocity can be formed for ions of the same $\mathrm{m} / \mathrm{z}^{10}$. Another issue with linear TOF MS is that not all the ions are formed at the same time or same location which would ultimately result in ions of the same $\mathrm{m} / \mathrm{z}$ will have different paths ${ }^{16}$. TOF instruments with a reflectron address those issues better, and now ions with the same $\mathrm{m} / \mathrm{z}$ ratio have a significantly better chance to reach the detector at the same time. The reason for this is that the reflectron, which has a homogeneous electric field, corrects for potential differences in kinetic energy of the same size of ions. This is even further amplified by orthogonal trajectories TOF MS instruments. This is because the initial ion velocity is minimized by collisional cooling and orthogonally injected ions are reaccelerated; this way the direction of ions entering the flight path is independent of $\mathrm{m} / \mathrm{z}^{17,18}$. Resolution up to 80,000 has been reported for orthogonal TOF MS instruments ${ }^{14,15}$. 


\subsubsection{Detector}

The detector in mass spectrometry is a device that can count the number of ions that are striking it and convert that signal to a mass spectrum. However, there is an only small number of ions that make it to the detector, so detectors need to be quite responsive to sense the signal. The electron multiplier detectors function by continuous dynode (semiconductor plate) arrangements ${ }^{4}$. This functions via collisions of ions coming out of MS analyzer with a semiconductor plate (e.g., copper/beryllium alloy oxide) ${ }^{4}$ and this emits secondary electrons. These dynodes are kept at high voltage ( $\mathrm{VV}$ magnitude) so that the secondary electrons can be accelerated and focused onto a sequential dynode ${ }^{8}$. This process is repeated until a gain of about $10^{6}$ electrons are released ${ }^{19}$. Finally, this amplified current value is converted to voltage and sequentially to intensity value by an analog-to-digital converter $(A D C)^{4}$.

\subsection{Triple quadrupole mass spectrometer (QqQ)}

A type of quadrupole $M S$ instrument is triple quadrupole instrument $(Q q Q)$, where three quadrupoles are placed one after another, as shown in Figure 2:5. In this kind of instrument fragmentation experiments are possible. In a fragmentation experiment, the first quadrupole is used to scan or filter the ions of interest (parent ions), the fragmentation of these parent ions happens in second quadrupole (creating daughter ions), and the third quadrupole scans these newly created daughter ions ultimately generating the fragmentation spectrum. The fragmentation in the second quadrupole is possible without the application of DC voltage; this is called RF-only mode ${ }^{8}$. RF-only mode can be used 
to transfer ions from one part of the instrument to another. Full mass scan experiments on the triple quadrupole instrument are possible by only scanning masses either in first or third quadrupole. More details on the triple quadrupoles will be given in the discussion of hybrid MS instruments.

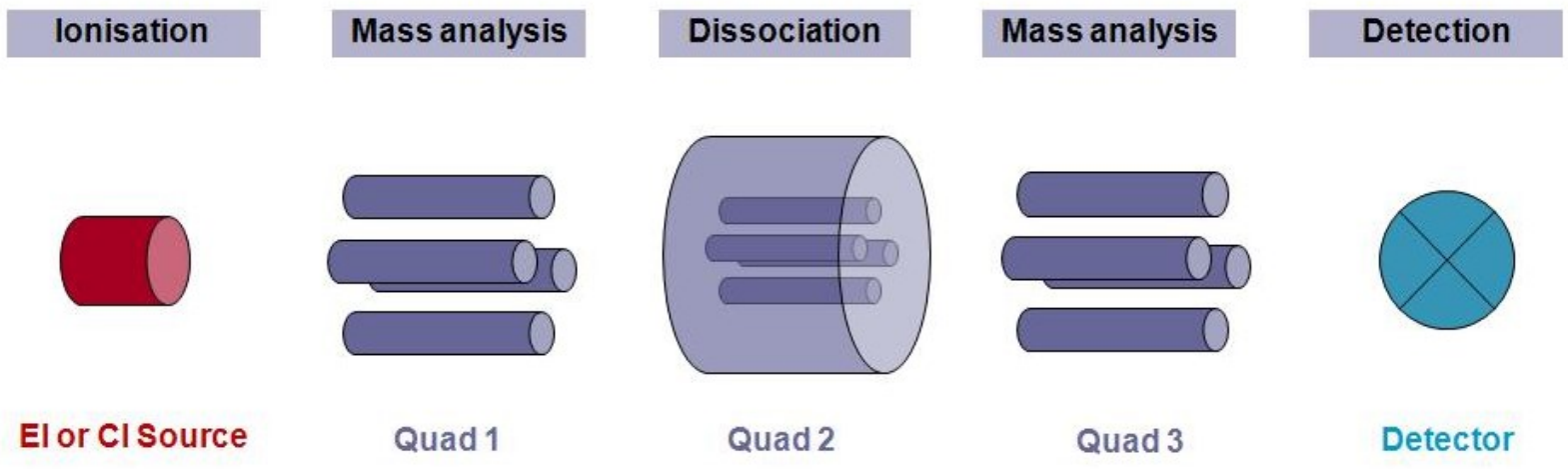

Figure 2:5. Schematic of a triple quadrupole (QqQ) analyzer. This Figure was adapted from https://www.biologie.hu-berlin.de/de/gruppenseiten/oekologie/meth/massspec/mass_sp

\subsection{Hybrid MS instrument used for this thesis work}

\subsubsection{AB Sciex QTRAP 4000}

AB Sciex QTRAP 4000 is a hybrid triple quadrupole linear ion trap instrument where the last quadrupole is an ion trap; a schematic for this instrument is shown in Figure 2:6. This instrument is also attached to an HPLC and comes with electrospray ionization (ESI). In this kind of instrument, generated ions are trapped in the last quadrupole. Ions are held in the trap under full control of the electric field. To inject an ion of interest frequency is changed, which causes the ion to become unstable and fall out of the trap and go towards the detector. 


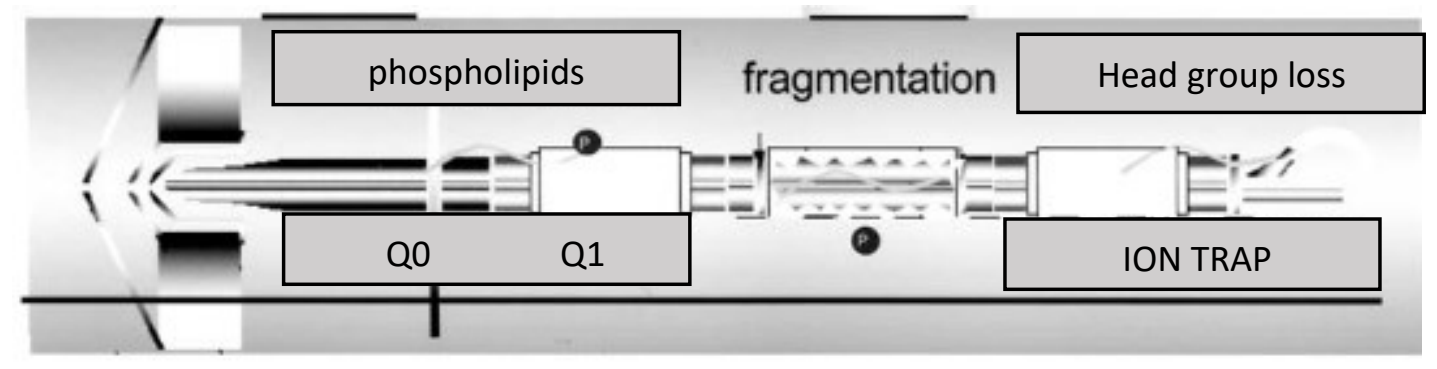

Figure 2:6. Schematic of a hybrid triple quadrupole linear ion trap instrument. This Figure was adapted from Cox et al., $2005^{20}$.

The first quadrupole (Q1) scans and filters ions of interest (parent ions), in the second quadrupole (q2), ions of interest are fragmented (creating daughter ions). Fragmentation in q2 happens via collision-induced dissociation (CID). The ions enter the q2 by applying an electric potential between $\mathrm{q} 0$ and $\mathrm{q} 2$ to have a defined kinetic energy, and then these ions are collided with neutral gas molecules to cause fragmentation; $q 2$ is also called a collision cell. Third quadrupole (Q3) can work in different modes; this enables one to use QTRAP 4000 in various kinds of experiments. The different modes of operation of the Q3 are summarized in table 2:1.

Table 2:1. Shown are various triple quadrupole linear ion trap operation modes. This table was adapted from Hopfgartner et al., 2004 21.

\begin{tabular}{|c|c|c|c|}
\hline Mode of operation & Q1 & q2 & lon trap \\
\hline Q1 Scan & Resolving (Scan) & RF-only & RF-only \\
\hline Q3 Scan & RF-only & RF-only & Resolving (Scan) \\
\hline Product lon Scan (PI) & Resolving (Fixed) & Fragment & Resolving (Scan) \\
\hline Precursor lon Scan (PC) & Resolving (Scan) & Fragment & Resolving (Fixed) \\
\hline Neutral Loss Scan (NL) & Resolving (Scan) & Fragment & Resolving (Scan Offset) \\
\hline $\begin{array}{c}\text { Selected Reaction } \\
\text { Monitoring mode } \\
\text { (SRM) }\end{array}$ & Resolving (Fixed) & Fragment & Resolving (Fixed) \\
\hline
\end{tabular}

Full mass scan experiments on the triple quadrupole ion trap instrument are possible by scanning masses either in first or third quadrupole only. The other two 
quadrupoles are in the RF-only mode for these scans which means they are just passing ions to the next section of the instrument. The QTRAP 4000 is also able to do product ion scan $(\mathrm{PI})$, precursor ion scan $(\mathrm{PC})$, neutral loss scan $(\mathrm{NL})$, selected reaction monitoring scan (SRM) and multiple reaction monitoring scan (MRM). MRM is not shown in Table $2: 1$.

A product ion scan (daughter ion scan) allows only one $\mathrm{m} / \mathrm{z}$ of interest to pass to q2, where it is fragmented, and daughter ions for the parent of the ion of interest are created, these daughter ions are then scanned through Q3 and allowed to the detector. A selected reaction monitoring scan is also like product ion scan in that it allows only one $\mathrm{m} / \mathrm{z}$ of interest to pass to q2. After fragmentation of the ion of interest, however, only one of the fragmented ions is allowed to Q3, where it is scanned through and allowed to the detector. MRM is essentially like SRM with multiple times of SRM for the same or different parent ions of interest. A schematic diagram for MRM monitoring is shown in Figure 2:7.

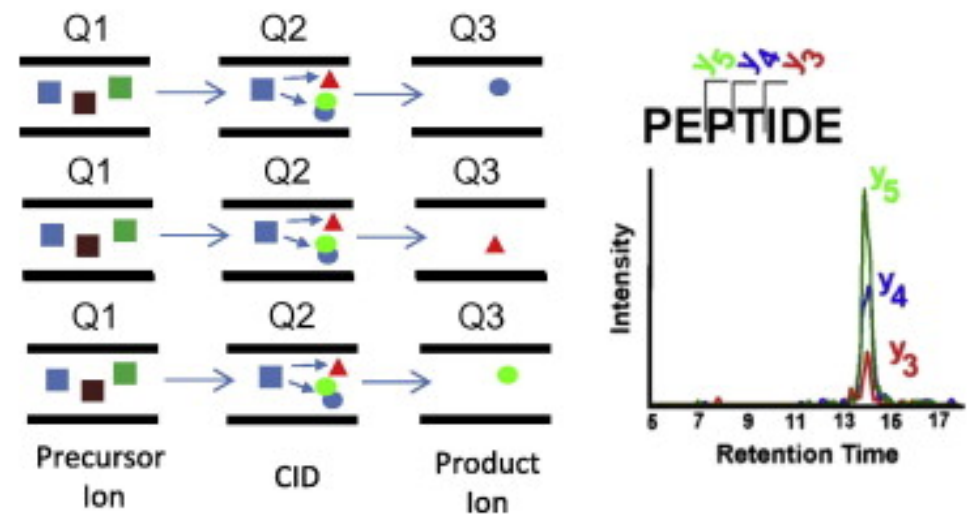

Figure 2:7. Schematic diagram of multiple reaction monitoring. This Figure was adapted from Colangelo et al., $2013^{22}$. 
The two modes of ion trap operation that were part of lipidomics studies for this thesis were precursor ion scanning and neutral loss scanning. These kinds of scans are used to find a group/class of molecules that all lose same ion or neutral fragment. Precursor ion scan (parent ion scan) is opposite of product ions scan. Here Q3 is fixed to allow only one of the fragment ions to pass. So, only those parent ions that will fragment to form that specific ion fragment of interest will be allowed to go to the detector (Q1 is scanned). For example, the phosphatidylcholine class of lipids have a head group of mass $184 \mathrm{Th}$. If the Q3 is set only to recognize 184 Th, only lipids that lose a head group of 184 Th will be recognized.

The neutral loss scan is a bit different since no charged mass fragment is lost. In this case, Q1 and Q3 are scanned together, and Q3 is looking for all the ions that have been offset by certain neutral mass from parent ions. An example for this is phosphatidylserine class of lipids which have a head group of mass $185 \mathrm{Da}$. After Q1 and Q3 are scanned, Q3 is scanning for all the ions that have been offset by 185 Da neutral loss from the parent ions and passes those to detector.

Like the quadrupole MS, the ion trap does not offer high resolution. The main limitation of an ion trap instrument is that if too many ions are trapped together at given time, it could cause a space-charge effect (continuous charge distribution over area or volume), which compromises resolution, sensitivity and mass accuracy ${ }^{8}$. However, the ion trap is advantageous in that there are quite of few modes in which it can function as described above. 


\subsubsection{Agilent 6550 iFunnel Q-TOF LC/MS}

This MS instrument is another hybrid instrument that is attached to an HPLC; the schematic for the 6550 Q-TOF is shown in Figure 2:8. It offers high-resolution detection of compounds up to 0.5 ppm mass accuracy, resolving power up to 40,000 and collection of up to 50 spectra per second ${ }^{23}$. The iFunnel technology allows for femtogram sensitivity and makes it easy to even detect compounds in trace amounts. The 6550 is equipped with dual electrospray jet stream ionization, iFunnel ion sampling technology, a hexapole collision cell, a beam shaper and analog to digital TOF detection.

The dual electrospray ionization (ESI), as the name implies, has two different ionization sources. The first one is connected to the HPLC and is sprayed into a chamber at an atmospheric pressure in the presence of a strong electrostatic field and heated drying gas; the second spray is for spraying reference mass ions. This ESI process is summarized in Figure 2:9.23

The capillary that ions enter after ESI process has six inlets and is called a hexabore capillary; this allows capture of up to $6 \mathrm{X}$ more ions compared to traditional instruments. The ions then enter the iFunnel; this is another new enhancement that Agilent has added to the instrument. The iFunnel is a two-stage ion funnel for ion enrichment and focusing. The first phase of the ion funnel operates at high pressure (714 Torr) where funnel voltages and radio frequency (RF) repel ions forward. The first stage funnel is offset from the second stage funnel inlet to prevent neutral atoms from entering downstream ion optics. The second phase ion funnel operates at lower pressure (1-3 Torr). This process is shown in Figure 2:10. ${ }^{23}$ 


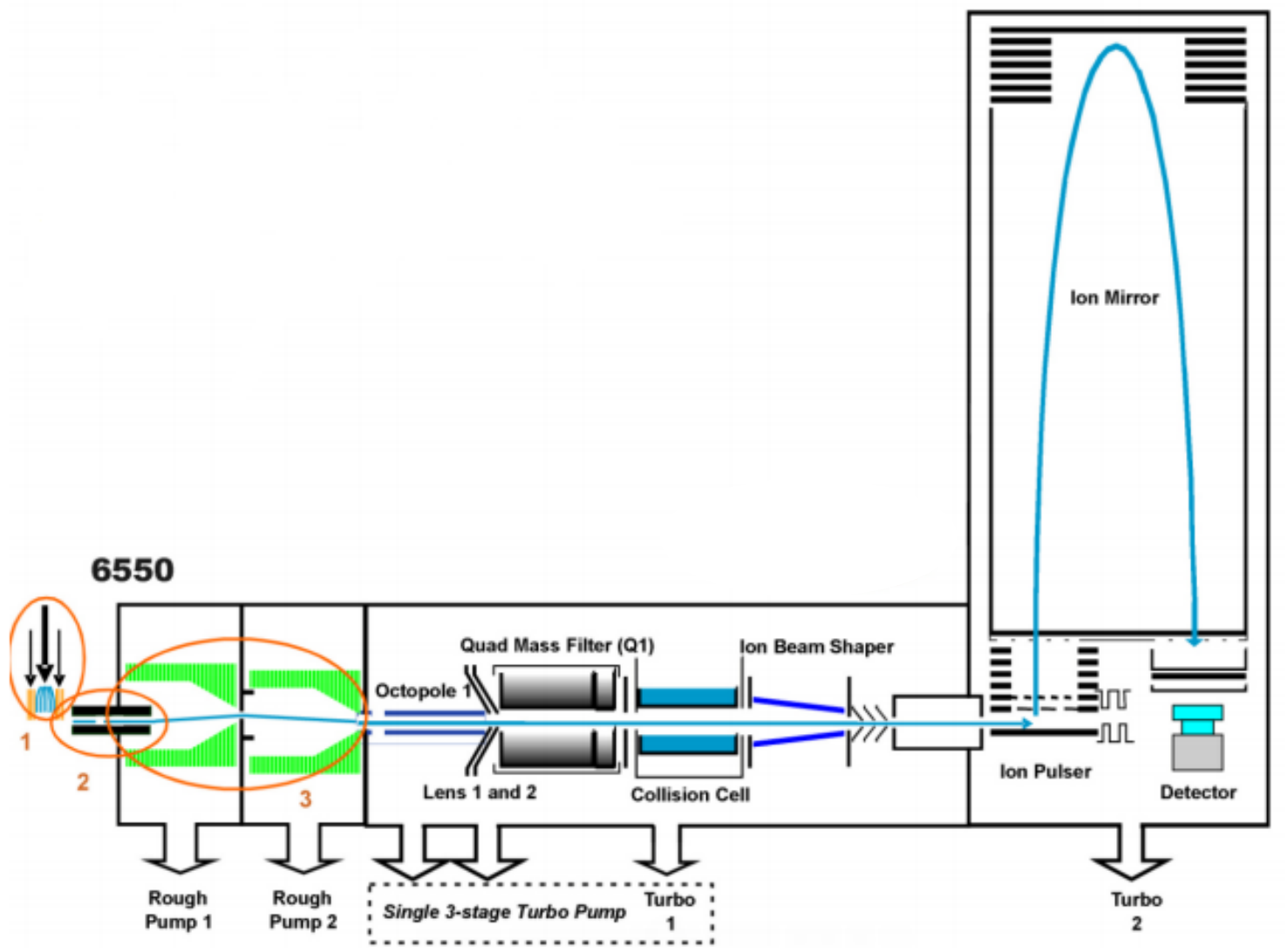

Figure 2:8. Schematic of Agilent iFunnel Q-TOF LC/MS (with major improvements to other instruments circled by Agilent). This Figure was adapted from the manual for the $6550^{23}$.

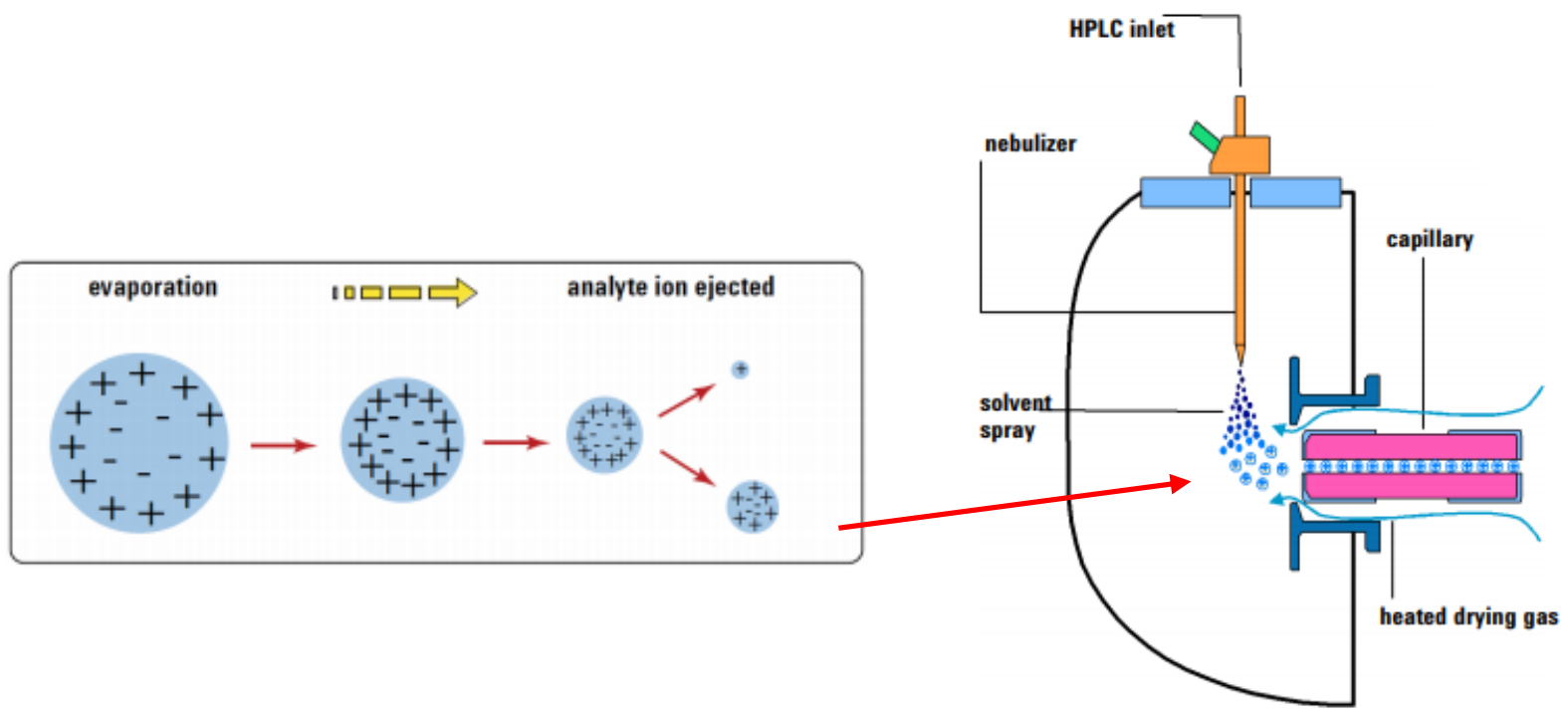

Figure 2:9. ESI in 6550 iFunnel Q-TOF LC/MS system. This Figure was adapted from Manual for $6550^{23}$. 


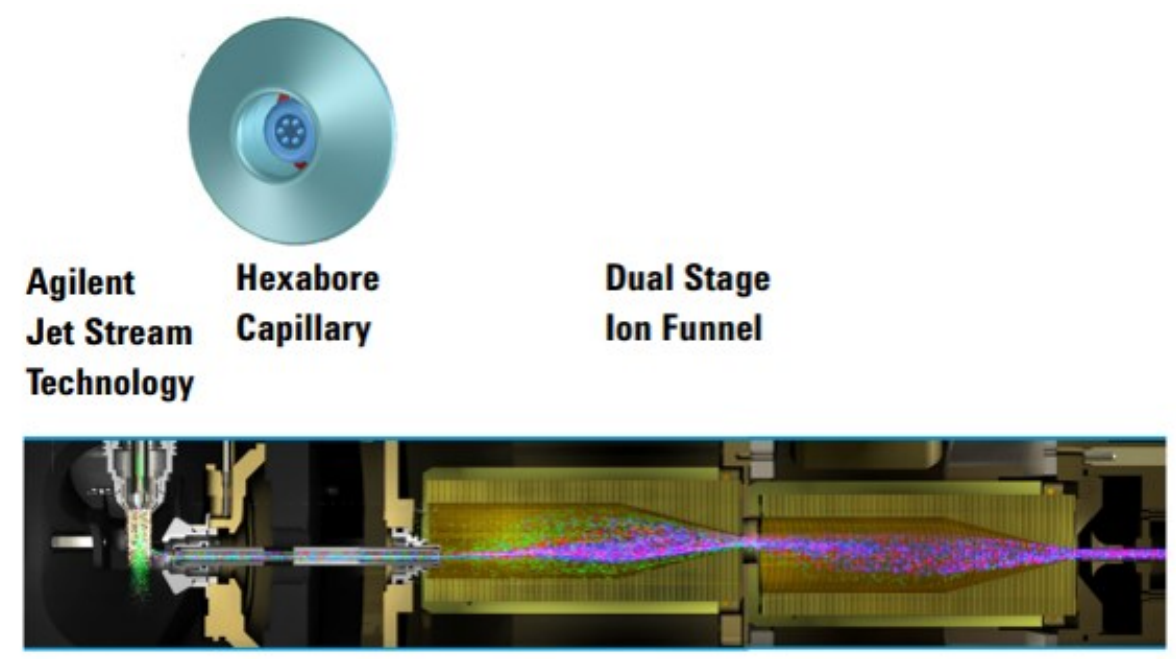

Figure 2:10. Hexabore capillary and dual stage ion funnel. This Figure was adapted from Manual for $6550^{23}$.

Following this, ions enter an octopole ion guide which allows almost $100 \%$ of the ions to be transmitted to quadrupole mass filter $(\mathrm{Q} 1)^{23}$. Here, only ions with the target mass are allowed to pass through. In a hexapole collision cell, precursor ions strike collision gas molecules generating daughter ions. After the collision cell, ions enter an ion beam shaper where the rods are converging towards the end so that more ions can reach the TOF ion accelerator, where they are accelerated and reflected via a reflectron to the detector.

\subsubsection{QStar XL from AB Sciex}

The QStar XL is a hybrid quadrupole-time-of-flight mass spectrometer; a schematic is shown in Figure 2:11. The short form for this instrument is $Q-T O F$, where $Q$ stands for quadrupole, q for quadrupole in RF-only mode, which is then connected to a time-of-flight ion separator. This instrument could be used in MS or MS/MS experiments; in MS/MS experiments q acts like collision cell and fragments parent ions via CID to 
daughter ions. After, the collision cell ions are collisionally cooled and reaccelerated to the TOF tube and finally collected on the detector. The Q-TOF has the option to be used with direct spray (nanospray tips can be used) or in conjugation with an HPLC. For research described in this thesis, the Q-TOF was used for proteomics studies.

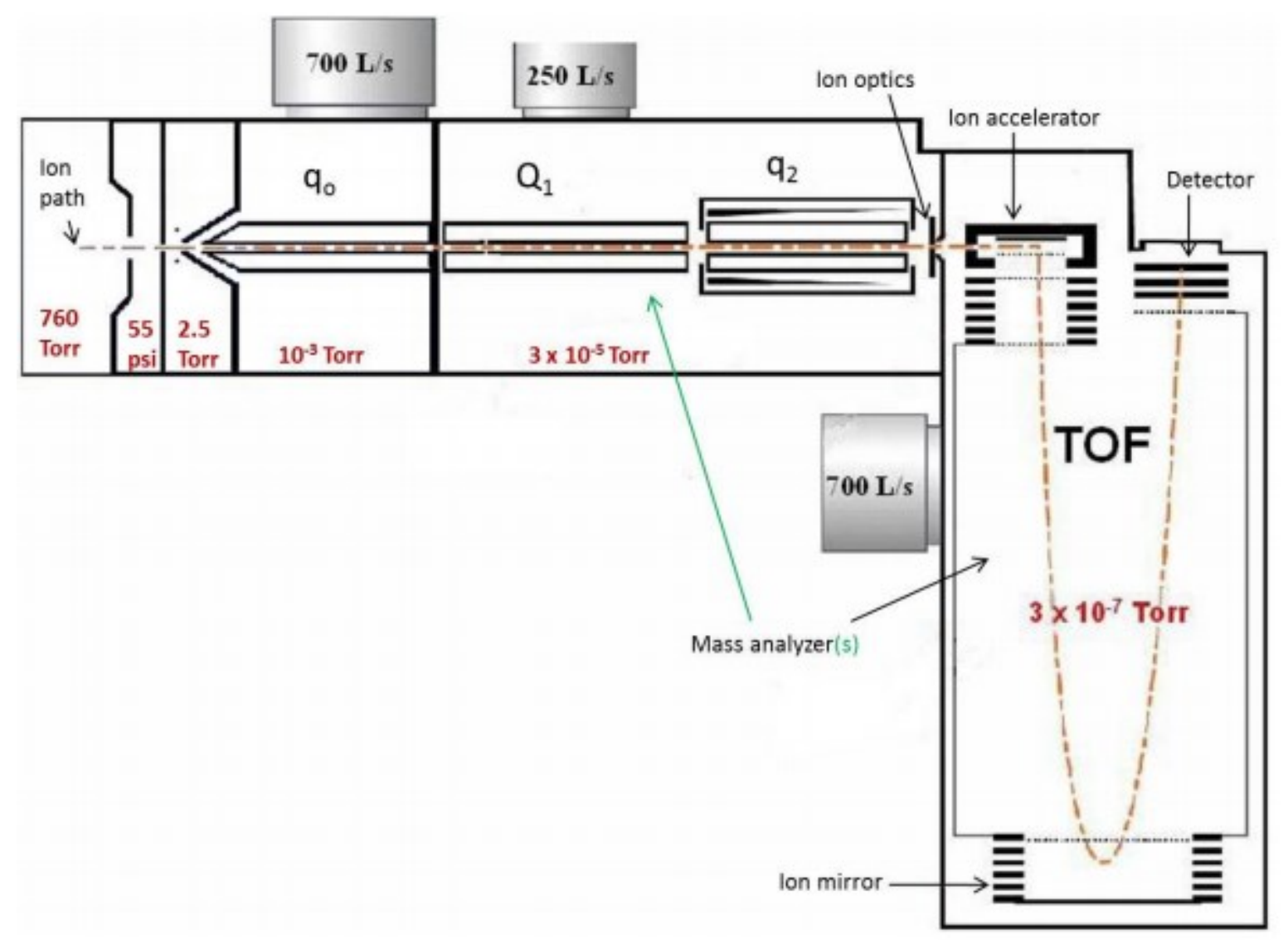

Figure 2:11. Schematic of a Q-TOF mass spectrometer. This Figure was adapted from Smith, $2005^{24}$. 


\subsection{Separation via high-performance liquid chromatography}

Chromatography techniques in conjugation with MS instruments can represent a robust combination of analytical tools. Chromatography techniques separate mixtures of compounds into individual components so that an MS can focus on analyzing only a few components at a time. This is especially useful when working with complex biological samples. Chromatography techniques also help to concentrate the individual components of the complex samples and help to clean up possible contaminants. Some examples of separation techniques include gas chromatography, liquid chromatography, ion mobility and capillary electrophoresis. However, HPLC was the only separatory technique used for this thesis work and will be the only one discussed.

HPLC consist of a stationary phase and a mobile phase. The stationary phase in HPLC is a solid, porous, surface-active material where sample mixture is adsorbed. The mobile phase in this separation technique is a solvent or combination of solvents. HPLC uses pumps to pass a pressurized liquid and a sample mixture through a column, resulting in the separation of the sample components.

\subsubsection{Reversed-phase HPLC}

Reversed-phase HPLC was used in this thesis work to separate lipids in conjugation with MS instruments. Reversed-phase HPLC uses a non-polar stationary phase to adsorb a mixture of compounds onto it. Analytes are typically eluted via a solvent gradient that begin with polar solvent(s) and becomes increasingly non-polar as the analysis progresses. This approach sees compounds elute according to their relative 
affinity for the mobile versus stationary phase, with analytes with high affinity for the mobile phase (more polar materials) eluting faster. Phospholipids that were separated with the HPLC are amphiphilic molecules; they were adsorbed to $\mathrm{C} 4$ stationary phase by the hydrophobic fatty acid chains. Solvents used to elute phospholipids were: solvent A (10 mM ammonium acetate in $30 \%$ methanol and MilliQ $\mathrm{H}_{2} \mathrm{O}$ ), solvent $\mathrm{B}(10 \mathrm{mM}$ ammonium acetate in $25 \%$ methanol and $75 \%$ IPA) and solvent C (100\% methyl tert-butyl ether (MTBE)) using gradient method over 50 minutes and flow rate $0.16 \mathrm{~mL} / \mathrm{min}$ (Figure 2:12). This HPLC separation was used on both instruments: the QTRAP 4000 and the 6550 Q-TOF with pressures to approximately 300 bar. The separatory columns were prepared by packing a fritted fused silica ( $200 \mu \mathrm{m}$ internal diameter (ID)) with C4 (5 $\mu \mathrm{m})$ to about $5.5 \mathrm{~cm}$.

\subsubsection{Strong cation exchange phase HPLC}

Strong cation exchange (SCX) phase HPLC was used in this thesis work in proteomics separations in conjugation with QSTAR MS instrument. SCX phase HPLC uses polar stationary phase to adsorb mixture of compounds onto it via electrostatic interactions and solvents of increasing ionic strength to elute the compounds from the column at their specific retention times (depending on their affinity to stationary phase) separating them. Trimethylated peptides that were separated with the HPLC have fixed positive charges; they were adsorbed to PolySULFOETHYL A stationary phase by the fixed positive sites on the peptides. Solvents used to elute these peptides were: solvent A $\left(0.1 \%\right.$ formic acid (FA) in $50 \%$ acetonitrile $(\mathrm{ACN})$ and $50 \%$ MilliQ $\left.\mathrm{H}_{2} \mathrm{O}\right)$ and solvent $B$ (200 mM triethylammonium acetate (TEAA) in 100\% MilliQ $\mathrm{H}_{2} \mathrm{O}$ ) using gradient method. 
The separatory columns were prepared by packing a fritted fused silica $(200 \mu \mathrm{m}$ ID) with a strong cation exchange $(\mathrm{SCX})$ resin $(12 \mu \mathrm{m})$ to about $10 \mathrm{~cm}$.

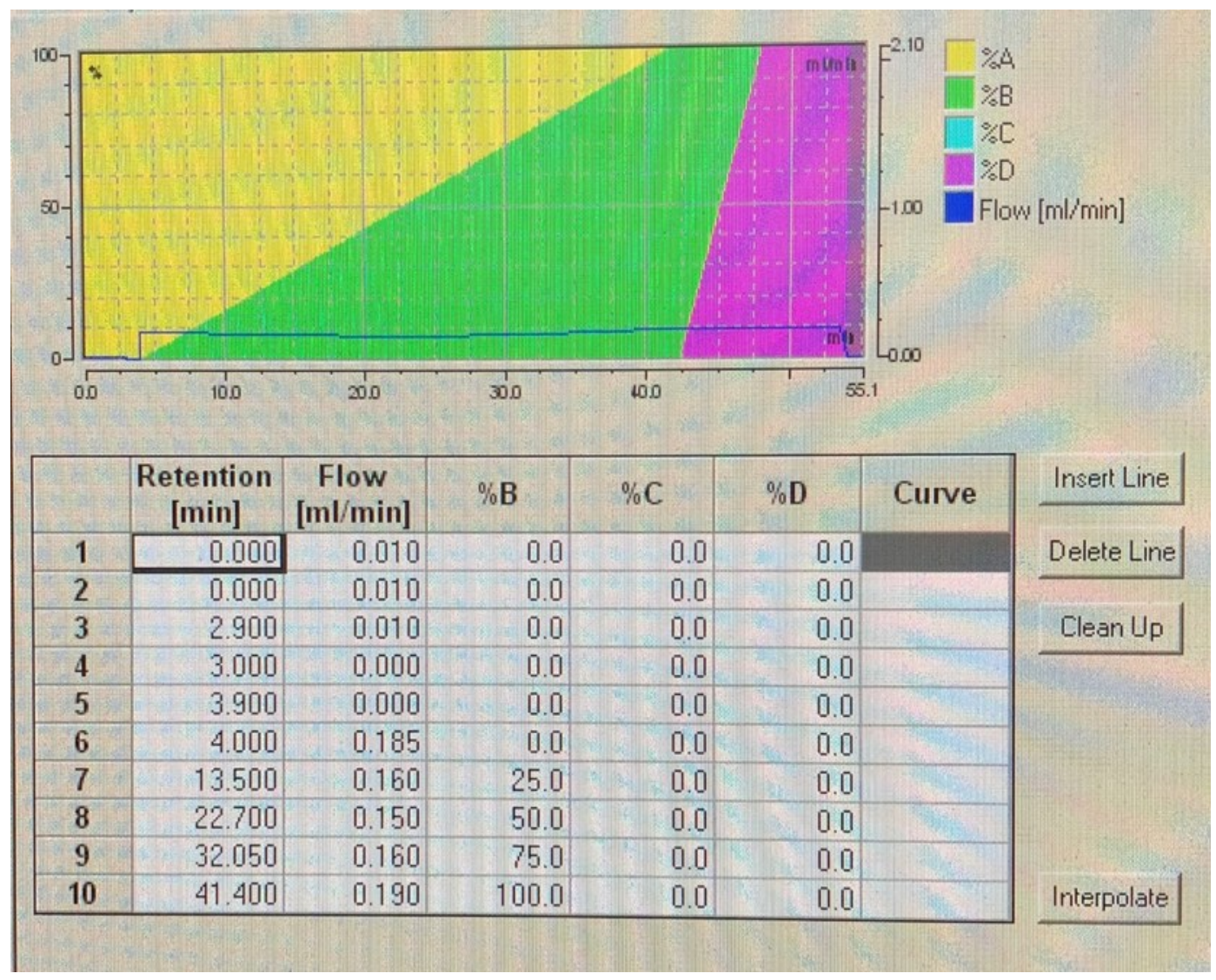

Figure 2:12. Visual representation of the gradient used in the phospholipid experiments.

\subsection{MS-based analysis of biological molecules}

\subsubsection{MS-based lipidomics}

Lipids have diverse structures; they can consist of various polar headgroups, fatty acid chain, backbone structures ${ }^{25}$. This structural diversity poses many challenges in lipid research, as it is challenging to find one approach to analyze them all. And quite often research is limited to only certain types of lipids or their classes. However, continuous 
advancements in the field of mass spectrometry have played a major role in growing lipidomics research with more sensitive and accurate ways of identifying and quantifying these species ${ }^{26-28}$. Rustam and Reid, 2018 showed that mass spectrometric techniques are the most widely used techniques in lipidomics research and it has been growing continuously since $2003^{29}$.

Figure 2:13 shows a typical MS-based workflow for lipidomic samples. It starts with a certain tissue or cell culture of interest. The tissue needs to be homogenized to allow for lipid extraction. After which lipids are stored but mostly analyzed as soon as possible since lipids oxidize and various structural alternations can form ${ }^{25}$. The workflow in the thesis for lipidomic work follows as shown in Figure 2:13 with the exception that antioxidants were not added as lipids were analyzed within a week or faster from extraction. Chromatography separation techniques are often used in tandem with MS analysis for better sample analysis. Most of the work in lipidomic research comes from data processing as data of complex biological samples is quite complex as well. There are also various lipidomic online resources available to facilitate the data processing. For this thesis work, Lipidomic Gateway Lipids Database (LIPID MAPS) was used.

The phospholipids of interest in this thesis are PCs, PEs, PSs and SMs (discussed in Chapter 1). These species have polar head groups that they lose during fragmentation in collision cell in MS. For this reason, a hybrid triple quadrupole linear ion trap is a very attractive instrument to use as these lipid classes can be relatively simply monitored and extracted. The ion trap scan and neutral loss scan can be used to identify these species in positive ion mode as summarized in table 2:2. PC and SM species lose fragment ion of 184 Th as they both lose choline head group. PE and PS lose the neutral mass of 141 
and 185 Da respectively and can be identified by these differences between parent and daughter ions. Other studies have investigated phospholipids by LC-MS/MS in the positive ion mode $28,30,31$.

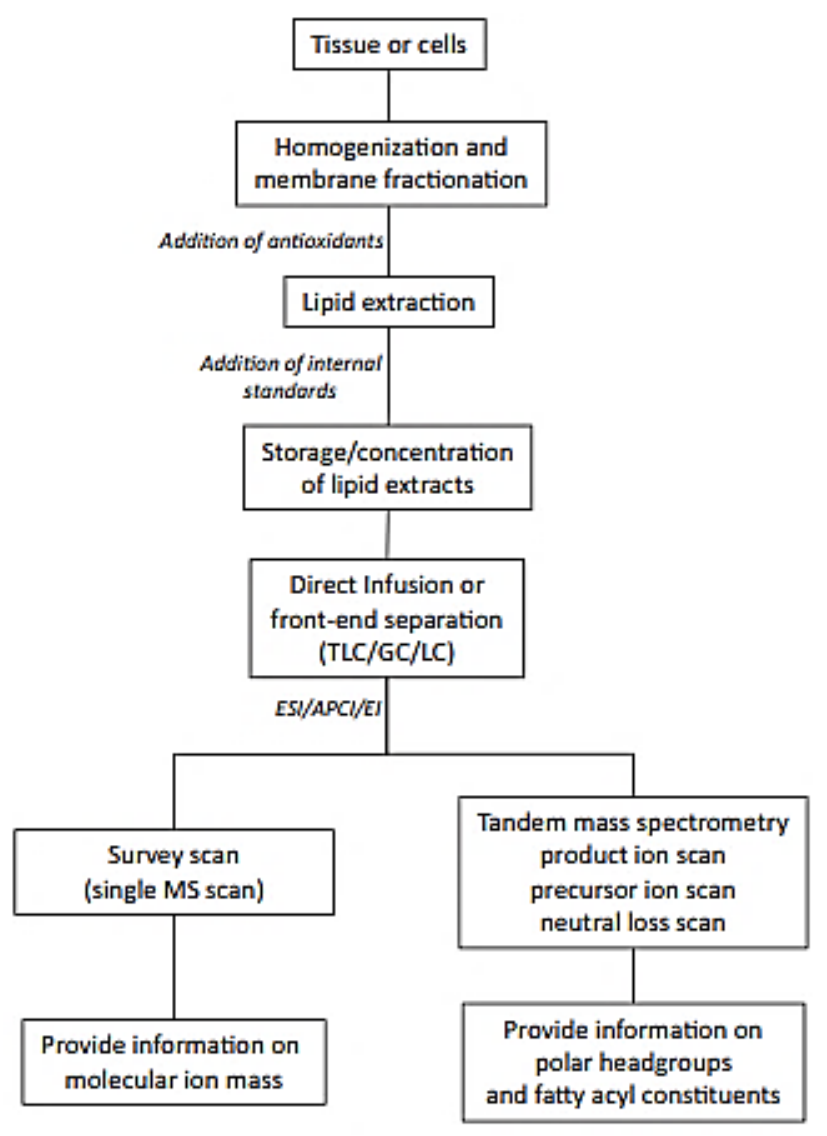

Figure 2:13. Typical workflow of MS-based lipidomics. This Figure was adapted from Khalil et al., $2010^{25}$. 
Table 2:2. Identification of phospholipids using tandem MS. This table is modified table 2 from Bandu et al., $2016^{28}$.

\begin{tabular}{cclc}
\hline Phospholipid (PL) & Major ion & Polarity & Identified part of PL \\
\hline PC & 184 Th & Positive & Loss of PC head group \\
\hline PE & $\left([\mathrm{M}+\mathrm{H}]^{+}-141\right)$ & Positive & Loss of PE head group \\
\hline PS & $\left([\mathrm{M}+\mathrm{H}]^{+}-185\right)$ & Positive & Loss of PS head group \\
\hline SM & 184 Th & Positive & Loss of PC head group \\
\hline
\end{tabular}

\subsubsection{Proteins and MS-based proteomics}

Some proteomics experiments have also been conducted in this thesis research and will be described in the last chapter. The individual amino acids (shown in Figure 2:14 with some chemical characteristics) are the building blocks of peptides and ultimately proteins. Amino acids are linked to each other by peptide bonds (Figure 2:15); the "linear" amino acid sequence forms a primary structure of proteins. Secondary (hydrogen bonding between residues), tertiary (the overall shape of one protein), and quaternary (structures formed by several protein subunits) structures of proteins are beyond the scope of this thesis ${ }^{32}$. Protein sizes vary; however, some larger ones are over $100 \mathrm{kDa}$ in size. It is tough to analyze whole proteins on MS, and thus they are mostly always digested by various enzymes to smaller peptide fragments. One of the most common digestion enzymes for proteomics research is trypsin, which cleaves on the C-terminal side of arginine $(R)$ and lysine $(K)$. However, trypsin is shown not to cleave if either arginine or lysine are followed by proline $(P)^{33}$. An example of horse heart myoglobin with its trypsindigested fragments are shown in Figure 2:16. 


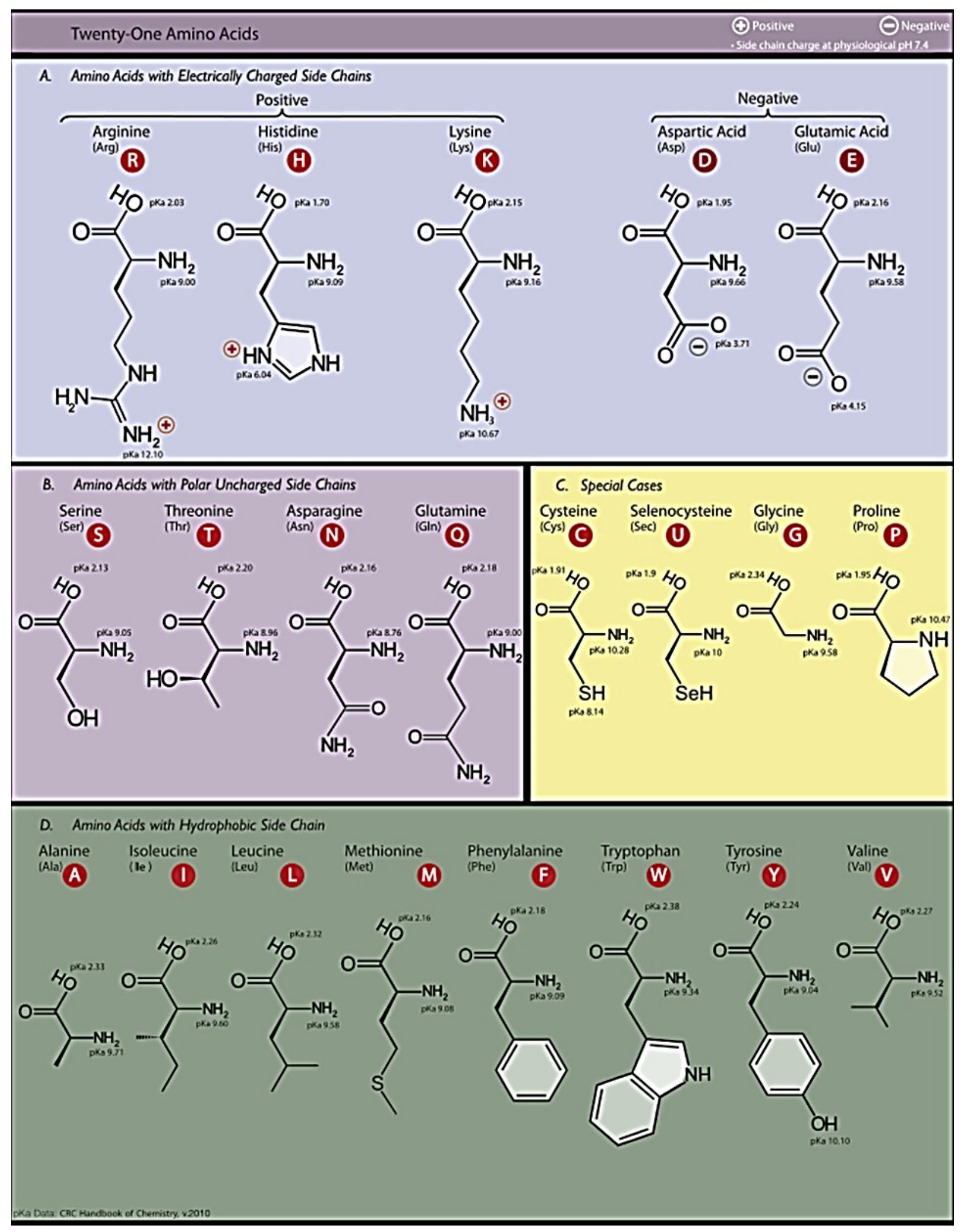

Figure 2:14. Amino acid structures. This Figure was adapted from https://en.wikipedia.org/wiki/Proteinogenic_amino_acid 


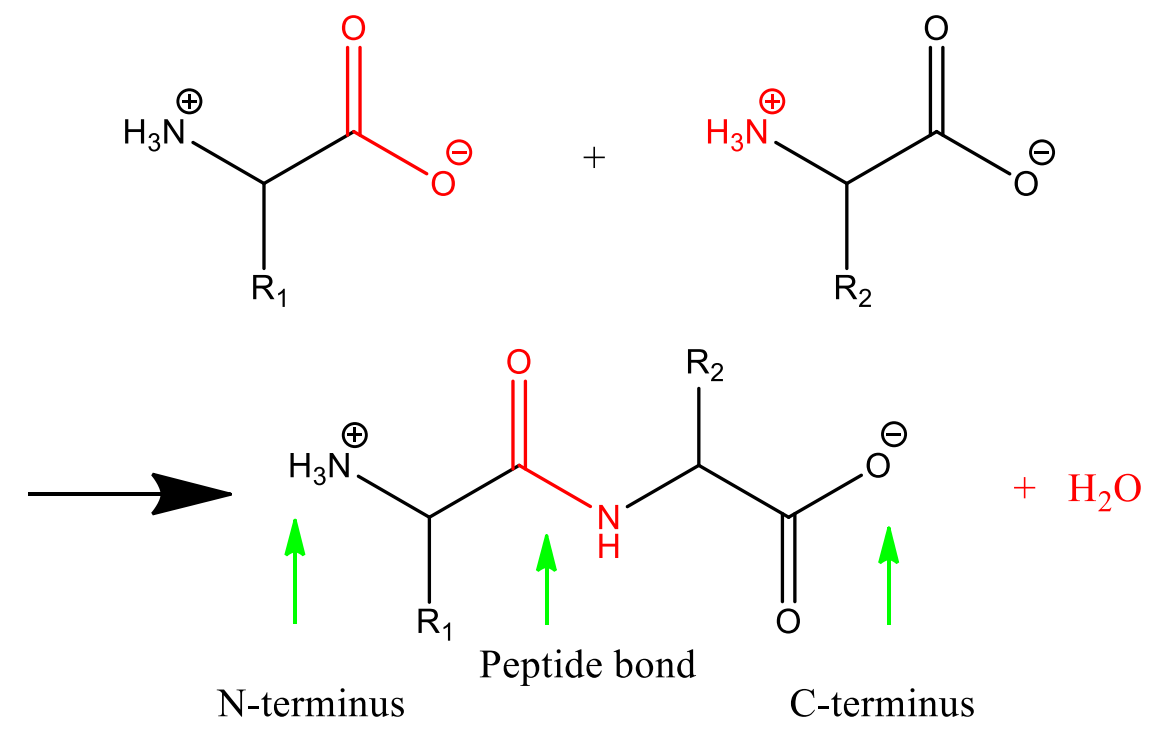

Figure 2:15. Peptide bond formation when two amino acids are combined.

\section{GLSDGEWQQVLNVWGKVEADIAGHGQEVLIRLFTGHP ETLEKFDKFKHLKTEAEMKASEDLKKHGTVVLTALGGIL KKKGHHEAELKPLAQSHATKHKIPIKYLEFISDAIIHVLH SKHPGDFGADAQGAMTKALELFRNDIAAKYKELGFQG}

Figure 2:16. Horse heart myoglobin protein amino acid sequence, colors indicate peptides observed after trypsin digestion.

Once the peptide fragments of the protein are obtained, they are subjected to MS analysis. In this thesis work, a Q-TOF instrument was used for proteomics work, which could analyze via both MS and MS/MS experiments. When Q1 is operated in an RF-only mode, MS experiments are performed. If MS/MS experiments are desired, Q1 selects the ion of interest and transmits it to the collision cell where CID is performed on the parent 
ions to obtain daughter ions. The possible fragmentation locations of a peptide are summarized in Figure 2:17. The charged ions from the C-terminus are $x-, y-, z$-ions depending on the location of the fragmentation as shown in Figure 2:17. The charged ions from the N-terminus are a-, b-, c-ions again depending on the location of the fragmentation. A-ions form when b-ions lose $\mathrm{CO}$ (mass of $28 \mathrm{Da}$ ). The numbers on the fragments $x, y, z$ and $a, b, c$ represent how many amino acid units are there in the ions. For example, in Figure 2:17 y2-ion contains two amino acid groups ( $R_{4}$ and $\left.R_{5}\right)$. This kind of nomenclature is used for peptide fragments.

CID fragmentation of the peptides has shown to mostly fragment peptides at their weakest bonds; which are the peptide bonds ${ }^{34}$. This means that mostly $y$ and b-ions are seen on peptide fragmentation spectra ${ }^{35}$. The loss of $28 \mathrm{Da}$ can occur for b-ions forming a-ions. However, this is mostly seen for $b_{2}$-ions forming characteristic $a_{2} / b_{2}$-ions pair ${ }^{34,36}$. Identification of $b$ and $y$-ions helps with amino acid sequence determination, and the more of peptides are identified, the better protein structure identification is recognized.

The typical proteomics mass spectrometry-based workflow is summarized in Figure 2:18. The final step, which is not shown in Figure 2:18, is usually matching the obtained spectrum to the proteomics database to see if a protein can be identified as well as the statistical significance of the identification. The Mascot is a software package that permits the automatic identification of peptide MS/MS data for proteomics and is commonly used by many research laboratories. This method of proteomics is known as the bottom-up approach; the top-down approach would investigate fragmentation of proteins rather than peptides. 
The use of an HPLC can aid in protein identification as that way peptide fragments are not introduced into MS instrument all at once. Usually, reversed phase HPLC is used with peptide separations, and they are eluted in order of their hydrophobicity with increasing organic solvent content in the gradient ${ }^{34,37}$.

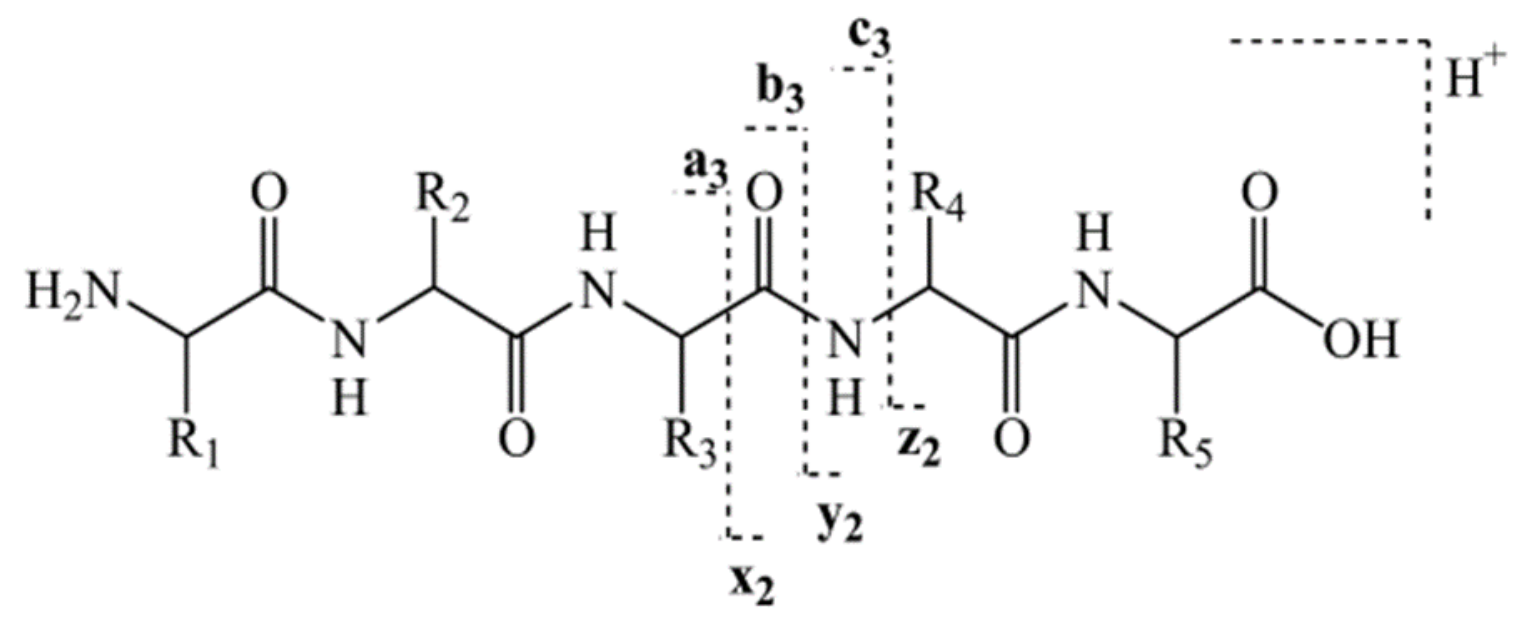

Figure 2:17. An example of peptide fragmentation locations and nomenclature. This Figure was adapted from Wysocki et al., 2005 ${ }^{38}$. 

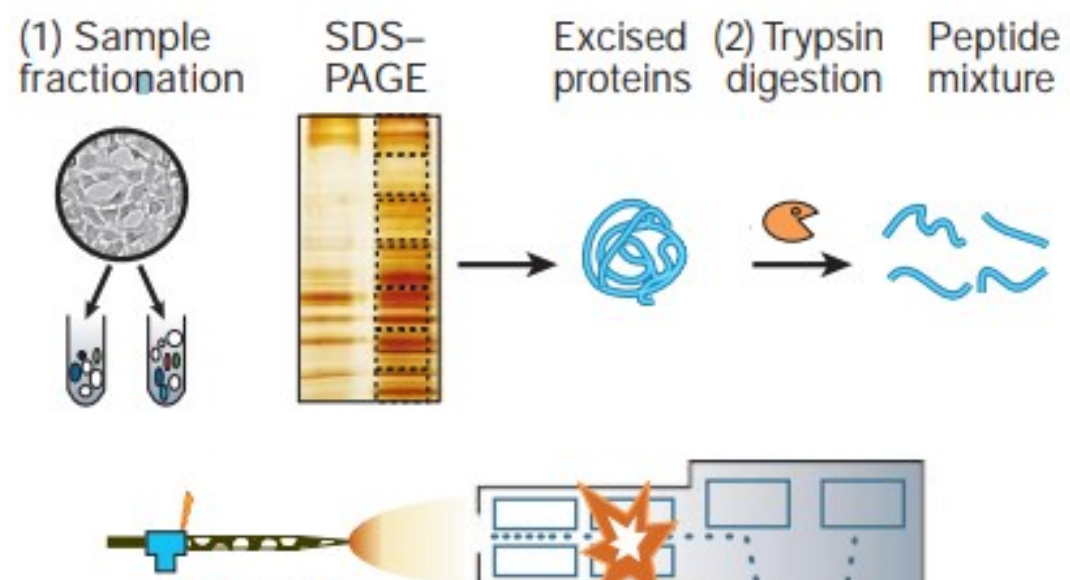

(3) Peptide chromatography and ESI

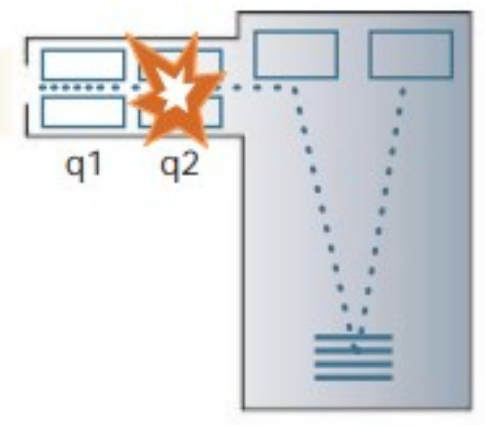

(4) MS

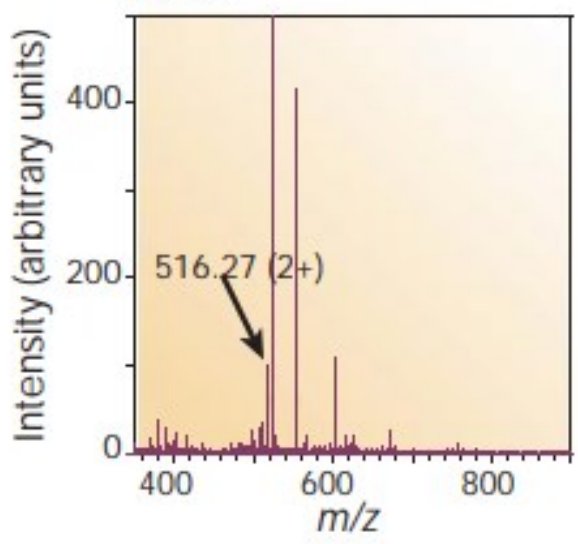

(5) MS/MS

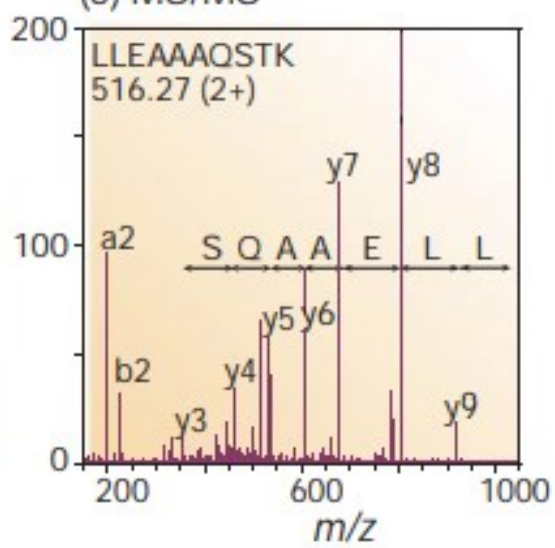

Figure 2:18. The typical workflow in MS-based proteomics. (1) Samples under analysis are fractioned to obtain a proteomic mixture which is separated (SDS-Page is one example of separation techniques) and proteins of interest are cut out from SDS-Page. (2) Protein of interest is digested to peptide fragments (trypsin is a common enzyme used for this procedure). (3) Peptide sample mix obtained is subject to MS and MS/MS experiments on Q-TOF instrument. (4 and 5) MS and MS/MS spectra are obtained and analyzed for peptides and ultimately for protein identification. This Figure was adapted from Aebersold and Mann, $2003^{39}$. 
The peptides of interest for this thesis are mainly phosphorylated and methylated peptides and that will be the focus in Chapter 7 . This kind of peptides are hard to analyze by MS as they appear on the spectrum with various charge states. Phosphorylated peptides are also hydrophilic and often zwitterionic which makes separation of these peptides on HPLC difficult and the identification on MS almost impossible. Chapter 7 will be focused on how to identify these peptides better. An overview will be given on some preliminary results. An optimization of the technique discussed is need in order to apply this technique in more complex peptide mixtures from proteins.

\subsection{References}

(1) Pavia, D. L.; Lampman, G. M.; Kriz, G. S. Introduction to spectroscopy, 3rd ed.; Vondeling, J., Kiselica, S., Eds.; Brooks/Cole: USA, 2001.

(2) Schwartz, J. C.; Senko, M. W.; Syka, J. E. P. Am. Soc. Mass Spectrom. 2002, 13, 659-669.

(3) Tang, X.; Bruce, J. E.; Hill, H. H.; Jr. Anal. Chem. 2006, 78 (22), 7751-7760.

(4) Banerjee, S.; Mazumdar, S. Int. J. Anal. Chem. 2012, 2012, 1-40.

(5) Wilm, M.; Mann, M. Anal. Chem. 1996, 68 (1), 1-8.

(6) Karas, M.; Bahr, U.; Dülcks, T. Fresenius. J. Anal. Chem. 2000, 366 (6-7), 669676.

(7) Demartini, D. R. In Tandem Mass Spectrometry - Molecular Characterization; Coelho, A. V., de Matos Ferraz Franco, C., Eds.; InTech, 2013.

(8) Christian, G. D.; Dasgupta, P. K.; Schug, K. A. Analytical Chemistry, 7th Ed.; Wiley: USA, 2014.

(9) Harris, D. C. Quantitative chemical analysis, 8th Ed.; W. H. Freeman: New York, 2010.

(10) Johnstone, R. A. W.; Rose, Malcolm, E. Mass spectrometry for chemists and biochemists, 2nd Ed.; Cambridge, University Press: Great Britain, 1996.

(11) Downard, K. Mass spectrometry a foundation course; The Royal Society of Chemistry: Sydney, Australia, 2004. 
(12) Stein, T.; Bandyopadhyay, B.; Troy, T. P.; Fang, Y.; Kostko, O.; Ahmed, M.; Head-Gordon, M. Proc. Natl. Acad. Sci. 2017, 114 (21), E4125-E4133.

(13) Bondarenko, P. V; Macfarlane, R. D. Int. J. Mass Spectrom. Ion Process. 1997, 160, 241-258.

(14) Dodonov, A. F.; Kozlovski, V. I.; Soulimenkov, I. V; Raznikov, V. V; Loboda, A. V; Zhen, Z.; Horwath, T.; Wollnik, H. Eur. J. Mass Spectrom 2000, 6, 481-490.

(15) Laiko, V. V.; Dodonov, A. F.; Cotter, R. J. Rapid Commun. Mass Spectrom. 1994, 8 (9), 720-726.

(16) Cai, Y.-H.; Lai, Y.-H.; Wang, Y.-S. J. Am. Soc. Mass Spectrom 2015, 26, 1722 1731.

(17) Chernushevich, I. V.; Ens, W.; Standing, K. G. Anal. Chem. 1999, 71 (13), 452A$461 \mathrm{~A}$.

(18) Krutchinsky, A. N.; Chernushevich, I. V.; Spicer, V. L.; Ens, W.; Standing, K. G. J. Am. Soc. Mass Spectrom. 1998, 9 (6), 569-579.

(19) Koppenaal, D. W.; Barinaga, C. J.; Denton, M. B.; Sperline, R. P.; Hieftje, G. M.; Schilling, G. D.; Andrade, F. J.; Barnes, J. H. Anal. Chem. 2005, 419-427.

(20) Cox, D. M.; Zhong, F.; Du, M.; Duchoslav, E.; Sakuma, T.; McDermott, J. C. J. Biomol. Tech. 2005, 16 (2), 83-90.

(21) Hopfgartner, erard; Varesio, E.; Tsc ap at, V.; Grivet, C.; Bourgogne, E.; Alexis Leuthold, L. J. MASS Spectrom. J. Mass Spectrom 2004, 39, 845-855.

(22) Colangelo, C. M.; Chung, L.; Bruce, C.; Cheung, K.-H. Methods 2013, 61 (3), 287-298.

(23) Manual. Agilent Technologies: Santa Carla, CA, USA 2014, p 132.

(24) Smith, J. C. Mass Spectrometry-Based Proteomics: Non-Covalent Interactions and Protein Identification, York University, Toronto, ON, 2005.

(25) Khalil, M. B.; Hou, W.; Zhou, H.; Elisma, F.; Swayne, L. A.; Blanchard, A. P.; Yao, Z.; Bennett, S. A. L.; Figeys, D. Mass Spectrom. Rev. 2010, 29, 877-929.

(26) Wang, C.; Kong, H.; Guan, Y.; Yang, J.; Gu, J.; Yang, S.; Xu, G. Anal. Chem. 2005, 77 (13), 4108-4116.

(27) Ren, C.; Liu, J.; Zhou, J.; Liang, H.; Wang, Y.; Sun, Y.; Ma, B.; Yin, Y. Lipids Health Dis. 2018, 17 (22).

(28) Bandu, R.; Mok, H. J.; Kim, K. P. Mass Spectrom. Rev. 2018, 37 (2), 107-138.

(29) Rustam, Y. H.; Reid, G. E. Anal. Chem. 2018, 90, 374-397.

(30) Retra, K.; Bleijerveld, O. B.; van Gestel, R. A.; Tielens, A. G. M.; van Hellemond, J. J.; Brouwers, J. F. Rapid Commun. Mass Spectrom. 2008, 22 (12), 1853-1862. 
(31) Brügger, B.; Erben, G.; Sandhoff, R.; Wieland, F. T.; Lehmann, W. D. Proc. Natl. Acad. Sci. U. S. A. 1997, 94 (6), 2339-2344.

(32) Millar, T. Biochemistry explained. A practical guide to learning biochemistry; Overseas Publishers Association: Singapore, 2000.

(33) Rodriguez, J.; Gupta, N.; Smith, R. D.; Pevzner, P. A. J. Proteome Res. 2008, 7 (1), 300-305.

(34) Steen, H.; Mann, M. Nat. Rev. Mol. Cell Biol. 2004, 5 (9), 699-711.

(35) Cottrell, J. S. J. Proteomics 2011, 74 (10), 1842-1851.

(36) Schlosser, A.; Lehmann, W. D. J. Mass Spectrom. 2000, 35 (12), 1382-1390.

(37) Hunt, D. F.; Yates lii, J. R.; Shabanowitz, J.; Winston, S.; Hauer, C. R. Chemistry (Easton). 1986, 83, 6233-6237.

(38) Wysocki, V. H.; Resing, K. A.; Zhang, Q.; Cheng, G. Methods 2005, 35, 211-222.

(39) Aebersold, R.; Mann, M. Nature 2003, 422, 198-207. 


\section{Chapter 3. Investigation of phospholipid structural dynamics between euthermic and hibernating squirrel liver tissue by LC/MS/MS}

\subsection{Abstract}

Northern climate animals have different ways of coping with long cold winters: squirrels hibernate, for example. Hibernation is a state that could be characterized by low body temperature, slow breathing and heart rate, and low metabolic rate. In the late summer and fall, before going into hibernation, squirrels eat a lot of food. Their bodies are able to live off the stored body fat through the winter. Lipids from the liver of hibernating and non-hibernating 13-lined ground squirrels were extracted using a modified Bligh and Dyer lipid extraction and analyzed via liquid chromatography/mass spectrometry. Our results indicated that the ground squirrel most likely increases the fluidity of its membranes during hibernation by increasing the degree of lipid unsaturation. This was evident through increase in concentration of highly unsaturated phospholipids. Increasing the unsaturation of the fatty acid chains prevents them from interacting too strongly and therefore increases the fluidity and permits continued cellular activity at low temperatures. Also, it was observed that mostly low abundance phospholipids that get upregulated in torpor. The identification experiments of phospholipids that change statistically significantly in concentration during cold months were performed. 


\subsection{Introduction}

Lipids constitute an abundant class of bio-macromolecules present in mammalian species $^{1}$. Lipids are involved in energy storage, are key components of the cellular membrane, and function as signaling molecules in signal transduction pathways ${ }^{2}$. They have been increasingly found to play critical roles in cell proliferation, apoptosis and regulation of membrane traffic ${ }^{3,4}$. Aberrant lipid dynamics have also been found to play a major role in abnormal cellular behavior that can result in diseases including various cancers; the investigation of these lipids can lead to valuable insight in clinical research ${ }^{5,6}$.

Glycerophospholipids (GPLs) are derivatives of sn-glycero-3-phosphoric acid with varying polar head group moieties at the $s n-3$ position and at least one O-acyl, O-alkyl or O-alkyl-1'-enyl residue at the $s n-1$ or $s n-2$ positions ${ }^{2,7,8}$. The three GPL classes: phosphatidylcholine (PC), phosphatidylethanolamine (PE) and phosphatidylserine (PS) are the predominant species of lipids present in mammalian cells ${ }^{2}$. Sphingomyelins are a class of sphingolipids that have a sphingosine backbone yet share a considerable degree of structural similarity with PCs, being 1-O phosphocholine ceramide derivatives and are often grouped as phospholipids ${ }^{2,9}$.

Lipidomics has significant and far-reaching implications for health and disease research. Investigations into altered cellular lipidomes have provided helpful insight into several disease states ${ }^{2,10,11}$. Lipid imbalances, improper metabolism, and dysfunctional signaling are associated with a host of illnesses, including various human cancers such as lung, breast and brain ${ }^{5}$, as well as Alzheimer's disease ${ }^{12}$, and other neurological diseases. 
A recent avenue of research within lipidomics has been the search to understand biochemically relevant changes in the lipidome that occur during hibernation in animal models. Hibernation is a state of prolonged hypometabolism characterized by reduced body temperature, reprioritization of cellular functions and preservation/stabilization of macromolecules crucial to survival throughout the hypometabolic state ${ }^{13}$. The 13 -lined ground squirrel (Ictidomys tridecemlineatus), a preferential model for hibernation studies, survives the winter months by entering into hibernation consisting of periods of reduced hypometabolic activity, known as torpor, where they reduce their body temperatures from their euthermic values $\left(\sim 37^{\circ} \mathrm{C}\right)$ down to $\sim 5^{\circ} \mathrm{C}$ while concurrently reducing their overall metabolic rates to about $5 \%$ of the euthermic rates ${ }^{14}$. This state of torpor can last anywhere from days to weeks, interspaced by brief periods of rewarming to a euthermic temperature which lasts from 12 to 24 hours ${ }^{15}$. During these time periods a squirrel consumes food that it hid away in the preparation for winter.

Animal models of hypometabolism have contributed to the development of practical methods for hypothermic storage of cells, tissues, and organs, as well as cryopreservation of cells and tissues (e.g., sperm, embryos, cornea, skin, heart valves, pancreatic islets). When cells undergo hypothermic storage, a variety of stressors are present including low temperatures, lower nutrient and oxygen levels ${ }^{16}$ all of which would also affect human cells during hypothermic storage. The study of changes to the lipidome and their impact on defending against these stressors could provide new insights into these mechanisms. Additionally, lipids are key signaling molecules, and changes in certain species could indicate a shift in cellular processes. As an example, it has been discovered that changes in the lipid membrane have an effect on temperature and basal 
metabolic rate regulation during hibernation, specifically in the mitochondria ${ }^{17}$. Another aspect of hibernation studies is the possibility of developing treatments for obesity and metabolic syndrome ${ }^{18}$. More abstractly, hibernation studies may open exciting new avenues that could lead to technologies that can extend the human lifespan and allow for prolonged periods of suspended animation. Consequently, the combination of hibernation and lipidomics studies is critical for fully elucidating these new realms of research.

The purpose herein is to investigate and quantify the phospholipidic changes between euthermic and hypothermic 13-lined ground squirrel liver tissue via HPLC-ESIMS/MS in an attempt to evaluate and interpret the changes in the composition of the lipid species and the resulting effect on membrane fluidity. The structural composition of significant phospholipids will be further elucidated using high-resolution HPLC-ESI/MS and MS/MS.

\subsection{Experimental Procedures}

13-lined ground squirrels were captured in the wild by licensed trappers from United States Department of Agriculture and transferred to research facility ${ }^{19}$. In the lab, they were individually housed in shoebox cages, they were kept at room temperature, and were fed standard rodent diet. For hibernation, the animals were transferred to dark

room kept at $5^{\circ} \mathrm{C}$. This allowed squirrels to transition naturally to torpor state. All animals were sacrificed and sampled according to protocols described by McMullen and Hallenbeck, $2010^{20}$. All hibernation experiments and animal housing were approved by 
the National Institute of Neurological Disorders and Stroke (NINDS) and animal care and use committee (ACUC). Once tissues of interest were collected, they were flash frozen and shipped to Carleton University.

There were two different liver samples used: control and hibernating liver tissues. The control liver was sampled from 13-lined ground squirrels with Tb (body temperature) of approximately $37^{\circ} \mathrm{C}$. The hibernating liver was sampled from 13-lined ground squirrels with a $\mathrm{Tb}$ near $5^{\circ} \mathrm{C}$. A mass of $0.1 \mathrm{~g}$ of control or hibernating 13-lined ground squirrel liver tissue (mass was measured from couple of livers) was crushed into small fragments and placed in $1 \mathrm{~mL}$ of $2 \%$ acetic acid in methanol $(\mathrm{AcMeOH})$ in an Eppendorf tube. The tube with the sample was placed in a small styrofoam box filled with ice. The sample (while still on ice) was sonicated using a 130-watt ultrasonic processor sonicator (model: VCX130, from Thomas Scientific, Swedesboro, NJ, USA). It was sonicated for 90 seconds with the pulse on for 20 s and pulse off for 10 s with $50 \%$ amplitude.

Lipid extraction: Lipid extraction was done by modified the Bligh and Dyer method ${ }^{21}$. The samples above were transferred to $10 \mathrm{~mL}$ extraction tubes ( $10 \mathrm{~mL}$ glass Kimble tube) with $3.2 \mathrm{~mL}$ of $0.1 \mathrm{M} \mathrm{Na}$ acetate (filtered). The Corning tubes were rinsed 3 times with 1 $\mathrm{mL}$ of $2 \% \mathrm{AcMeOH}$, and each rinsing was transferred to the extraction tube (total volume of $2 \% \mathrm{AcMeOH}$ is $4 \mathrm{~mL}$ ). A volume of $60 \mu \mathrm{L}$ of $800 \mu \mathrm{M}$ internal standard (C13:0 lysophosphatidylcholine) was added to the extraction tubes. Then $3.8 \mathrm{~mL}$ of chloroform was added to the extraction tubes. The extraction tubes were vortexed, swirled and inverted $3 \mathrm{X}$ and placed into a centrifuge for 2 minutes at $2000 \mathrm{rpm}$ and $4^{\circ} \mathrm{C}$. The bottom phase was collected via Pasteur pipette and transferred to collection tubes $(10 \mathrm{~mL}$ glass Kimble 
tube). Extraction was repeated 3 more times with $2 \mathrm{~mL}$ of chloroform each time; all the bottom phases were retained in the same collection tubes. All of the chloroform was then evaporated under a constant stream of nitrogen gas until just dark yellow oily residue remained. The tissue samples were resuspended in $1200 \mu \mathrm{L}$ of absolute EtOH (anhydrous, $99.5 \%$ ). The samples were then incubated at $30^{\circ} \mathrm{C}$ for 10 minutes to dissolve lipids and centrifuged for 1 minute at $2000 \mathrm{rpm}$ and $4^{\circ} \mathrm{C}$. The samples were diluted $40 \mathrm{X}$ in absolute $\mathrm{EtOH}$ and then transferred to an amber glass tube, flushed with $\mathrm{N}_{2}$ gas and stored in a $-20^{\circ} \mathrm{C}$ freezer until analyzed.

HPLC-ESI-MS/MS analysis: $88 \mu \mathrm{L}$ of Kasil\#1 was mixed with $16 \mu \mathrm{L}$ of formamide in an Eppendorf tube and vortexed for a couple of minutes. About $15 \mathrm{~cm}$ long fused silica tubes with an inner diameter of $200 \mu \mathrm{m}$ were dipped to this Kasil/formamide mixture; silica tubes were left overnight to dry allowing frit to polymerize (coated tip downwards). These fritted columns were packed with $5.5 \mathrm{~cm}$ of $5 \mu \mathrm{m} \mathrm{C} 4$ silica beads using a nitrogen pressure vessel and then fritted at the other end as well. This way prepared silica column was used as a reverse phase column for lipids separation. Control technical triplicates were run on one column and hibernation technical replicates were run on second column.

The following HPLC solvents were used: solvent A (10 mM ammonium acetate in $45 \%$ methanol $/ 55 \%$ MilliQ water), solvent B (10 mM ammonium acetate in $100 \%$ IPA), and solvent D (methyl tert-butyl ether (MTBE)). The gradient began at $100 \%$ solvent A and gradually increased to $100 \%$ solvent B in 41.4 minutes, the run ended in MTBE and by 48 minutes it was at $100 \%$ solvent $D$ (total run time was 60 minutes). 
The sample mixture for separation and analysis on the HPLC-ESI-MS was prepared by using $12.5 \mu \mathrm{L}$ of the diluted extracted lipid sample, $10 \mu \mathrm{L}$ of absolute $\mathrm{EtOH}$ and $27.5 \mu \mathrm{L}$ of MilliQ water. The sample $(20 \mu \mathrm{L})$ was injected into the column using an HPLC (Dionex Ultimate 3000) auto-sampler, which was held at room temperature. The sample was introduced into the hybrid triple quadrupole linear ion trap mass spectrometer (AB Sciex QTRAP 4000) using electrospray ionization source with an electrode with an inner diameter of $100 \mu \mathrm{m}$.

A precursor ion scan of $\mathrm{m} / \mathrm{z} 184$ at low resolution was used to identify and quantify lipids with a PC headgroup. The low-resolution scan was from $\mathrm{m} / \mathrm{z} 400$ to 1000 . A precursor ion scan of $\mathrm{m} / \mathrm{z} 184$ at unit resolution was done for SM identification and quantification to differentiate between PCs and SMs; scan was from m/z 600 to 900 . At low-resolution, neutral loss scans of $141 \mathrm{Da}$ and $185 \mathrm{Da}$ were used to identify and quantify PE and PS, respectively. The neutral loss scan of 141 Da was from m/z 300 to 900 while the neutral loss scan of 185 Da was from $\mathrm{m} / \mathrm{z} 400$ to 1100 . All scans were done in positive ion mode, and triplicate analyses were done for the control and torpor samples.

After each new set of triplicates produced, the XICs of LPC 454, PC 760, PC 732 and PE 744 were investigated to ensure that their elution profiles were acceptable and that there is not a significant variability between different in-house columns and their performance.

LC/MS and LC/MS/MS high-resolution experiments were carried out on an Agilent 6550 Q-TOF. The following HPLC solvents were used: solvent A (10 mM ammonium acetate in $45 \%$ methanol $/ 55 \%$ MilliQ water), solvent $B(10 \mathrm{mM}$ ammonium acetate in 
$100 \%$ IPA. The instrument was run in positive ion mode for high mass accuracy identification and in negative ion mode for fatty acid identification.

Data analysis and statistical analysis: Analyst 1.5 .1 software was used to acquire the data which then was exported to MultiQuant 2.1.1 software (AB Sciex, Framingham, MA, USA). The different PIS and NL scans determined glycerophospholipid polar head group identity. Peak area measurements were performed on the extracted ion chromatographs of each species using MultiQuant 2.1.1. Relative quantitation was achieved by dividing the area of each found lipid by the area of the added internal standard. To ensure isotopic distribution was not erroneously mistaken for different $\mathrm{m} / \mathrm{z}$ lipids, all phospholipids possessing the same corrected retention time had to have area values $20 \%$ higher than the potential precluding $\mathrm{M}+2$ isotopic area. $\mathrm{PC}$ and $\mathrm{SM}$ species with the same retention time had to have area values $20 \%$ higher than the potential precluding $M+1$ isotopic area. For PC (or SM) species that have a preceding SM (or PC) species, the $\mathrm{M}+1$ area of the preceding lipid was subtracted to correct for isotopic contamination. Fold change was calculated by dividing the area of each lipid in the torpor sample to its counterpart in the control sample. Statistically significant changes were assessed using student's t-test $(\alpha=0.05)$ followed by Benjamini-Hochberg $(B H)$ correction $(\alpha=0.01)$ to control for false discovery rates. High-resolution MS data was analyzed by MassHunter Qualitative Analysis B.07.00 software. Lipid database Lipidomics Gateway at (www.lipidmaps.org) was used for lipid identification. 


\subsection{Results and Discussion}

Our lab has previously performed extensive work on the glycerophospholipidome of different cell culture lipid extracts and other animal tissues. The affordable cost of inhouse columns allows the user to prevent lipid carry-over between studies due to their disposable nature at the cost of smaller binding capacities and decreased chromatographic performance. An optimal 1:3 loading ratio (1 part sample in ethanol to 3 parts of water) was established for our $5.5 \mathrm{~cm} \mathrm{C4}$ or C18 in-house columns using a mobile phase of $30 \% \mathrm{MeOH}$ and $70 \%$ water with $10 \mathrm{mM}$ ammonium acetate 22 . For a variety of animal tissue and cell culture phospholipid extracts the polarity of the mobile phase was effective in allowing lipids, including very polar lipids, to readily bind to the stationary phase while keeping other lipids soluble. After several attempts of analyzing the squirrel liver control extracts with the triple quadrupole instrument, it became evident that the loading phase was too polar and was causing lipid solubility issues. The total ion chromatogram obtained with the Q-TOF instrument revealed that the content of glycerolipids (GL) in the sample was greater than the glycerophospholipid (GPL) content (Figure 3:1). The most abundant GPLs like PC and PE have stronger ionization efficiencies than glycerolipids, and yet the glycerolipid peak is more prominent, which indicates that GLs are the predominant lipid category in the sample. The solubility problems were resolved by loading the sample with a 1:1.22 loading ratio (1 part sample in ethanol to 1.22 parts of water) with a mobile phase of $45 \% \mathrm{MeOH}, 55 \% \mathrm{H}_{2} \mathrm{O}$ and 10 $\mathrm{mM}$ ammonium acetate. 


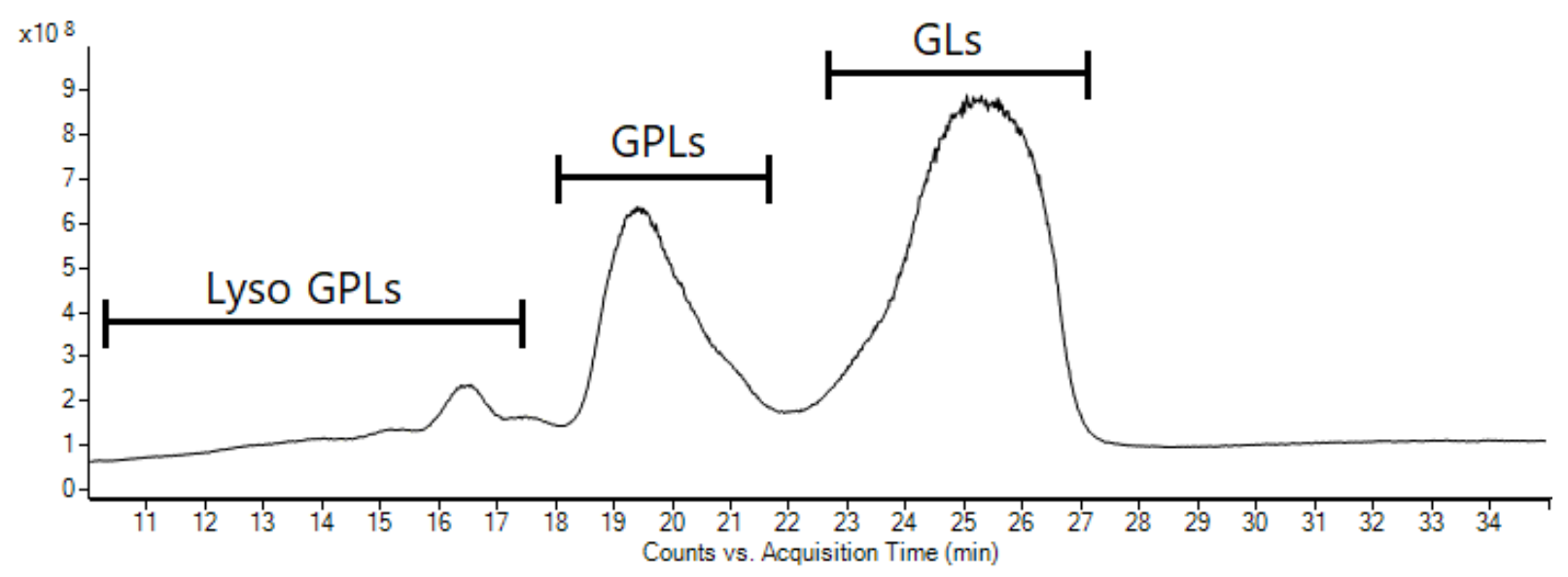

Figure 3:1. Total ion chromatogram of one of the control sample replicates acquired via reversed-phase LC-ESI-MS. Lysoglycerophospholipids and other polar lipids are the first ones to elute. Most diradyl GPLS elute between $18 \mathrm{~min}$ and $22 \mathrm{~min}$. Many diacylglycerols and most of the triacylglycerol species elute between minute 22.5 and minute 27. The predominant lipid species present in the spectrum are GLs despite many GPLs having better ionization efficiencies.

One of the most common challenges when doing lipid identification or quantitation is different lipids belonging to different classes often have the same exact mass. For instance $[\mathrm{PC}(\mathrm{O}-35: 1)+\mathrm{H}]^{+},\left[\mathrm{PC}(\mathrm{P}-35: 0+\mathrm{H}]^{+},[\mathrm{PE}(\mathrm{O}-38: 1)+\mathrm{H}]^{+},[\mathrm{PE}(\mathrm{P}-38: 0)+\mathrm{H}]^{+},[\mathrm{PA}(\mathrm{O}-\right.$ $\left.40: 2)+\mathrm{NH}_{4}\right]^{+}$and $\left[\mathrm{PA}\left(\mathrm{P}-40: 1+\mathrm{NH}_{4}\right]^{+}\right.$all have the same exact mass of $760.6215 \mathrm{Da}$. One solution to this mass overlap is the introduction of chromatographic separation before ionization. This will allow the unequivocal separation of PA species from potential isobaric PC and PE species (chromatographic methods greatly reduce ion suppression as well, thus improving lipid sensitivities). In mammalian studies, odd chained lipids are erroneously assumed to be absent. Careful chromatographic separation can manage to separate isobaric PC species from their PE counterparts as long as they are saturated. Saturated PCs elute slightly earlier than saturated PEs. Chromatographic separation of desaturated and isobaric PCs from PEs proves more complicated, as the position of the unsaturation(s) can result in identical elution profiles. Moreover, if one of the isobaric 
species is much more abundant than its isobaric counterpart (in mammalian samples usually the even chained lipids are much more abundant), the less abundant lipid could be buried under the more abundant lipid's chromatogram. To properly identify and quantify all lipids including very low abundance odd phospholipids we conducted our quantitation experiments via head group specific tandem MS scans. Figure 3:2 exemplifies the benefits of quantitating low abundance lipids whose area would erroneously be attributed to a more abundant lipid. The most abundant PC in both control and torpor samples was $\mathrm{PC}(34: 2)$ (Fig. 3:2A) and its peak eclipses and engulfs its PE(37:2) counterpart (Fig. 3:2C).

For phospholipid classes that have an efficient fragmentation pathway after collision-induced dissociation (CID) such as PC, SM, PE and PS, these scans efficiently reduce noise level and thus increase analyte sensitivity. The NL of $141 \mathrm{Da}$ exclusive to PEs allowed the extracted ion chromatogram (EIC) of the two PEs with $\mathrm{m} / \mathrm{z}$ of 758 to be identified and quantified. All limit of detection (LoD) and limit of quantification (LoQ) values were calculated as described in the supplementary section of Canez et al., $2016^{22}$ which was based on the original publication by Armbruster and Pry, $2008^{23}$. 

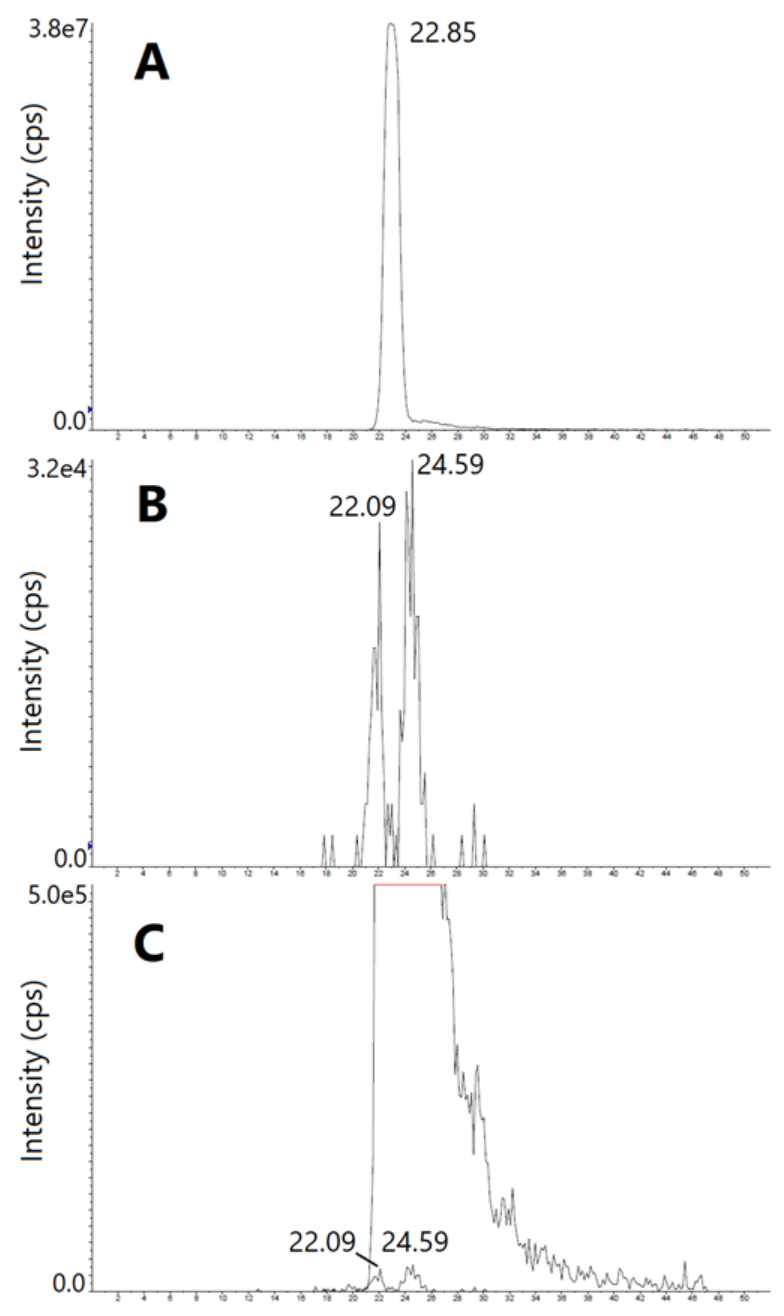

Time (min)

Figure 3:2. Panel A presents the extracted ion chromatogram (EIC) of 758 Th PC acquired via a PIS of 184 Th, from a torpor replicate. The PIS of 184 Th enabled the exclusive quantitation of PC (34:2) without isobaric interference. Panel B presents the EIC of two eluting PEs with $\mathrm{m} / \mathrm{z}$ of 758 acquired. The EIC was acquired using NL scan of 141 Da from a torpor replicate and enabled the identification and exclusive quantitation of the two PE species. The peak with a retention time (Rt) of 22.09 min corresponds to PE (38:9), and the peak with an Rt of 25.59 min corresponds to PE (37:2). Panel Coverlays the elution profiles of the PIS 184 Th and NL of 141 Da. The PC (34:2) peak has the biggest area of all PC species in the sample. Both PE areas are slightly above their respective LoQ. Panel C demonstrates that it would have been impossible to identify or quantify PE (37:2) with a high-resolution MS since it shares the same $\mathrm{m} / \mathrm{z}$ of 758.5694 as the dwarfing PC. The PE (38:9) would have been able to be identified as it has a different mass (758.4755 Th). 


\subsubsection{Triple quadrupole linear ion trap quantitation}

The PIS of 184 Th revealed a total of 89 PC species in the torpor sample, all above the LoQ. By contrast 79 PC species were found in the control sample above the LoQ, while the remaining 10 peaks were below the LoQ (BLoQ) but above the LoD. All peaks presented in Table S. 3:1. The peak at 454 Th is internal standard. Every statistically significant PC peak experienced a fold increase during torpor. The fold increases were calculated by dividing the torpor standardized average area by the control standardized average area. The smallest fold increase was $1.60 \mathrm{x}$ between the torpor and control 676 $\mathrm{m} / \mathrm{z}$ PC while the biggest fold increase was $23.19 x$ by 788 Th PC (Table 3:1). Fold change and student's t-test $p$-values were calculated for all $\mathrm{m} / \mathrm{z}$ values identified including average areas whose values were below the LoQ. Due to the inherent uncertainty provided by values below the limit of quantification, the presented values are not accurate and are only meant to showcase a rough estimation of the fold change. Therefore these 10 peaks are labeled in Table 3:1 as appeared (APP) instead.

Table 3:1. Statistically significant $P C, S M, P E$ and PS peaks after student's t-test and BH false discovery rate correction.

\begin{tabular}{|c|c|c|c|c|c|c|c|c|}
\hline \multirow[b]{2}{*}{$\mathbf{m} / \mathbf{z}$} & \multicolumn{3}{|c|}{ Control } & \multicolumn{3}{|c|}{ Torpor } & \multirow[b]{2}{*}{ P-value } & \multirow[b]{2}{*}{$\begin{array}{c}\text { Fold } \\
\text { change }\end{array}$} \\
\hline & $\begin{array}{l}\text { Standardized } \\
\text { average } \\
\text { retention } \\
\text { time }(\mathrm{min}) \\
\end{array}$ & $\begin{array}{l}\text { Standardized } \\
\text { average area }\end{array}$ & $\begin{array}{l}\text { Standard } \\
\text { deviation }\end{array}$ & $\begin{array}{l}\text { Standardized } \\
\text { average } \\
\text { retention } \\
\text { time (min) }\end{array}$ & $\begin{array}{l}\text { Standardized } \\
\text { average area }\end{array}$ & $\begin{array}{l}\text { Standard } \\
\text { deviation }\end{array}$ & & \\
\hline \multicolumn{9}{|c|}{ Phosphatidylcholine } \\
\hline 454 & 0.0 & $1.00 \mathrm{E}+00$ & $0.00 E+00$ & 0.0 & $1.00 \mathrm{E}+00$ & $0.00 E+00$ & & 1.00 \\
\hline 676 & 7.9 & $3.02 \mathrm{E}-01$ & $3.80 \mathrm{E}-02$ & 8.0 & $4.84 \mathrm{E}-01$ & $1.06 \mathrm{E}-02$ & 0.0013 & 1.60 \\
\hline 728 & 10.0 & \multicolumn{2}{|c|}{ 2.64E-02 BLoQ } & 10.5 & $9.04 \mathrm{E}-02$ & $1.18 \mathrm{E}-02$ & 0.0009 & APP (3.42) \\
\hline 732 & 11.0 & $6.63 E+00$ & $9.60 \mathrm{E}-01$ & 11.1 & $1.47 \mathrm{E}+01$ & $1.89 E+00$ & 0.0027 & 2.22 \\
\hline 744 & 10.9 & $7.75 \mathrm{E}-01$ & $1.06 \mathrm{E}-01$ & 10.9 & $2.20 \mathrm{E}+00$ & $3.14 \mathrm{E}-01$ & 0.0017 & 2.84 \\
\hline 754 & 10.8 & $4.16 \mathrm{E}-01$ & $7.19 \mathrm{E}-02$ & 10.6 & $1.39 E+00$ & $2.04 \mathrm{E}-01$ & 0.0014 & 3.35 \\
\hline 756 & 10.8 & $6.33 E+00$ & $8.86 \mathrm{E}-01$ & 10.8 & $2.08 \mathrm{E}+01$ & $2.11 \mathrm{E}+00$ & 0.0004 & 3.29 \\
\hline 766 & 11.2 & $8.89 E-01$ & $2.17 \mathrm{E}-01$ & 11.3 & $2.24 \mathrm{E}+00$ & $1.81 \mathrm{E}-01$ & 0.0012 & 2.52 \\
\hline 770 & 11.5 & $7.20 \mathrm{E}-01$ & 8.37E-02 & 11.3 & $2.56 \mathrm{E}+00$ & $3.43 \mathrm{E}-01$ & 0.0008 & 3.55 \\
\hline
\end{tabular}




\begin{tabular}{|c|c|c|c|c|c|c|c|c|}
\hline 772 & 9.0 & $6.64 \mathrm{E}-02$ & $1.88 \mathrm{E}-02$ & 9.0 & $1.23 \mathrm{E}+00$ & $6.28 \mathrm{E}-02$ & 0.0000 & 18.46 \\
\hline 774 & 12.4 & $2.83 \mathrm{E}+00$ & $3.11 \mathrm{E}-01$ & 12.2 & $7.54 \mathrm{E}+00$ & $8.14 \mathrm{E}-01$ & 0.0007 & 2.67 \\
\hline 774 & 9.1 & $1.60 \mathrm{E}-01$ & $3.89 E-02$ & 9.3 & $1.55 \mathrm{E}+00$ & $3.38 \mathrm{E}-01$ & 0.0021 & 9.73 \\
\hline 788 & 8.1 & 4.74E-02 & $8.14 \mathrm{E}-03$ & 8.1 & $1.10 \mathrm{E}+00$ & $1.80 \mathrm{E}-01$ & 0.0005 & 23.19 \\
\hline 790 & 7.6 & \multicolumn{2}{|c|}{ 3.51E-02 BLoQ } & 7.6 & $1.73 \mathrm{E}+00$ & $3.17 \mathrm{E}-01$ & 0.0008 & APP (49.12) \\
\hline 798 & 9.3 & $7.12 \mathrm{E}-02$ & $2.80 \mathrm{E}-02$ & 9.3 & $8.98 \mathrm{E}-01$ & $8.24 \mathrm{E}-02$ & 0.0001 & 12.62 \\
\hline 800 & 9.5 & $1.09 \mathrm{E}-01$ & $2.68 \mathrm{E}-02$ & 10.3 & $1.97 \mathrm{E}+00$ & $4.77 \mathrm{E}-01$ & 0.0025 & 18.12 \\
\hline 802 & 10.1 & $3.69 \mathrm{E}-01$ & $5.46 \mathrm{E}-02$ & 10.3 & $1.51 \mathrm{E}+00$ & 7.93E-02 & 0.0000 & 4.10 \\
\hline 814 & 13.4 & \multicolumn{2}{|c|}{ 1.46E-02 BLoQ } & 13.7 & $3.59 \mathrm{E}-01$ & 7.11E-02 & 0.0011 & APP (24.60) \\
\hline 816 & 14.5 & \multicolumn{2}{|c|}{ 3.32E-02 BLoQ } & 14.7 & 7.67E-01 & $1.76 \mathrm{E}-02$ & 0.0000 & $\operatorname{APP}(23.11)$ \\
\hline 818 & 8.9 & \multicolumn{2}{|c|}{ 2.98E-02 BLoQ } & 8.9 & $8.41 \mathrm{E}-01$ & $3.44 \mathrm{E}-02$ & 0.0000 & APP (28.25) \\
\hline 820 & 9.3 & \multicolumn{2}{|c|}{ 1.33E-02 BLoQ } & 8.9 & $1.96 \mathrm{E}-01$ & $2.57 \mathrm{E}-02$ & 0.0003 & APP (14.74) \\
\hline 824 & 13.0 & 3.37E-01 & $2.92 \mathrm{E}-02$ & 11.9 & 7.09E-01 & $4.48 \mathrm{E}-02$ & 0.0003 & 2.10 \\
\hline 826 & 13.0 & $1.80 \mathrm{E}-01$ & $4.41 \mathrm{E}-02$ & 12.6 & $6.33 \mathrm{E}-01$ & $5.24 \mathrm{E}-02$ & 0.0003 & 3.51 \\
\hline 828 & 13.1 & 2.67E-01 & $3.91 \mathrm{E}-02$ & 13.0 & 7.02E-01 & 7.44E-02 & 0.0009 & 2.63 \\
\hline 830 & 11.2 & $8.44 \mathrm{E}-01$ & $1.44 \mathrm{E}-01$ & 11.2 & $1.95 \mathrm{E}+00$ & $2.02 \mathrm{E}-01$ & 0.0015 & 2.31 \\
\hline 840 & 13.3 & $1.06 \mathrm{E}-01$ & $2.11 \mathrm{E}-02$ & 11.5 & $2.75 \mathrm{E}-01$ & $2.14 \mathrm{E}-02$ & 0.0006 & 2.58 \\
\hline 846 & 12.5 & $7.78 \mathrm{E}-02$ & $1.37 \mathrm{E}-02$ & 12.3 & $2.29 \mathrm{E}-01$ & $3.13 \mathrm{E}-02$ & 0.0016 & 2.94 \\
\hline 848 & 13.0 & $2.91 \mathrm{E}-01$ & $3.29 \mathrm{E}-02$ & 12.7 & $5.22 \mathrm{E}-01$ & $5.36 \mathrm{E}-02$ & 0.0031 & 1.79 \\
\hline 850 & 12.9 & $1.52 \mathrm{E}-01$ & $1.49 \mathrm{E}-02$ & 12.6 & $4.20 \mathrm{E}-01$ & $2.26 \mathrm{E}-02$ & 0.0001 & 2.76 \\
\hline \multicolumn{9}{|c|}{ Sphingomyelin } \\
\hline 701 & 10.0 & $1.08 \mathrm{E}-01$ & $2.71 \mathrm{E}-02$ & 10.1 & $2.91 \mathrm{E}-01$ & $2.88 \mathrm{E}-02$ & 0.0013 & 2.71 \\
\hline 729 & 11.0 & 2.87E-01 & $5.01 \mathrm{E}-02$ & 11.1 & $8.35 \mathrm{E}-01$ & $9.96 \mathrm{E}-02$ & 0.0010 & 2.91 \\
\hline \multicolumn{9}{|c|}{ Phosphatidylethanolamine } \\
\hline 758 & NA & \multicolumn{2}{|c|}{ 3.80E-03 BLoD } & 10.2 & $8.12 \mathrm{E}-02$ & $1.06 \mathrm{E}-02$ & 0.0003 & APP \\
\hline \multicolumn{9}{|c|}{ Phosphatidylserine } \\
\hline 834 & 11.0 & \multicolumn{2}{|c|}{ 1.61E-02 BLoQ } & 11.1 & $1.38 \mathrm{E}-01$ & $1.42 \mathrm{E}-02$ & 0.0001 & APP (8.57) \\
\hline
\end{tabular}

APP - The average area of the peak in the control sample was either below the limit of detection or the limit of quantitation

BLoQ- Below the limit of quantitation

BLoD-below the limit of detection

In this study we used two brand new in-house columns for the torpor and control studies in order to completely eliminate any cross-contamination between the control and torpor samples, eliminating the potential for compromising quantitation. Since the torpor triplicates used a different column than the control triplicates, their retention times were 
shifted by a minute. The internal standard was used to correct this shift by subtracting all individual retention times by the retention time of the LPC (13:0) standard and averaging triplicate values. The internal standard was the first phospholipid to elute. This internal standard was chosen as this lysolipid was not found in tissues under investigation and would not interfere with any lipids present in the sample, also it was more cost effcient adding only one internal standard.

Several early eluting upregulated peaks shared their mass with a non-significant PC counterpart that had a longer elution time. Three examples are presented in Figure 3:3. The left column shows on top the EIC of 772 Th during the control sample and on the bottom its equivalent EIC of 772 Th during torpor. The earlier eluting peak is present in both control and torpor sample but is clearly upregulated in the torpor sample. The later eluting peak is non-significant. The control and torpor EIC of 790 Th (middle column Fig. 3:3) and EIC of 820 Th (right column Fig 3:3), also demonstrate that the earlier eluting peak is significantly upregulated. During control, the earlier eluting peak of both 790 and 820 Th is BLoQ while their torpor counterpart is above the LoQ. The concentration of the later eluting peaks in the 790 and 820 Th EIC spectra did not change in a statistically significant manner. From the 28 statistically significant peaks a total of 10 peaks $(772$, $788,790,798,800,802,814,816,818$ and $820 \mathrm{Th}$ ) were peaks that eluted earlier than their same mass non-statistically significant PC counterparts. The only instance where both peaks (corrected retention times (cRt) of $9.3 \mathrm{~min}$ and $12.1 \mathrm{~min}$ ) were statistically significant and had the same mass, were the two peaks present in the EIC of $774 \mathrm{Th}$. Out of the previously mentioned 11 peaks, 8 of them $(676,772,774,788,790,798,818$ and $820 \mathrm{Th}$ ) had exceptionally early elution profiles with cRts lesser than $10 \mathrm{~min}$. These torpor 
upregulated statistically significant peaks with early elution profiles represent highly hydrophilic PC species that would be increasing membrane fluidity during torpor by disrupting packing density.

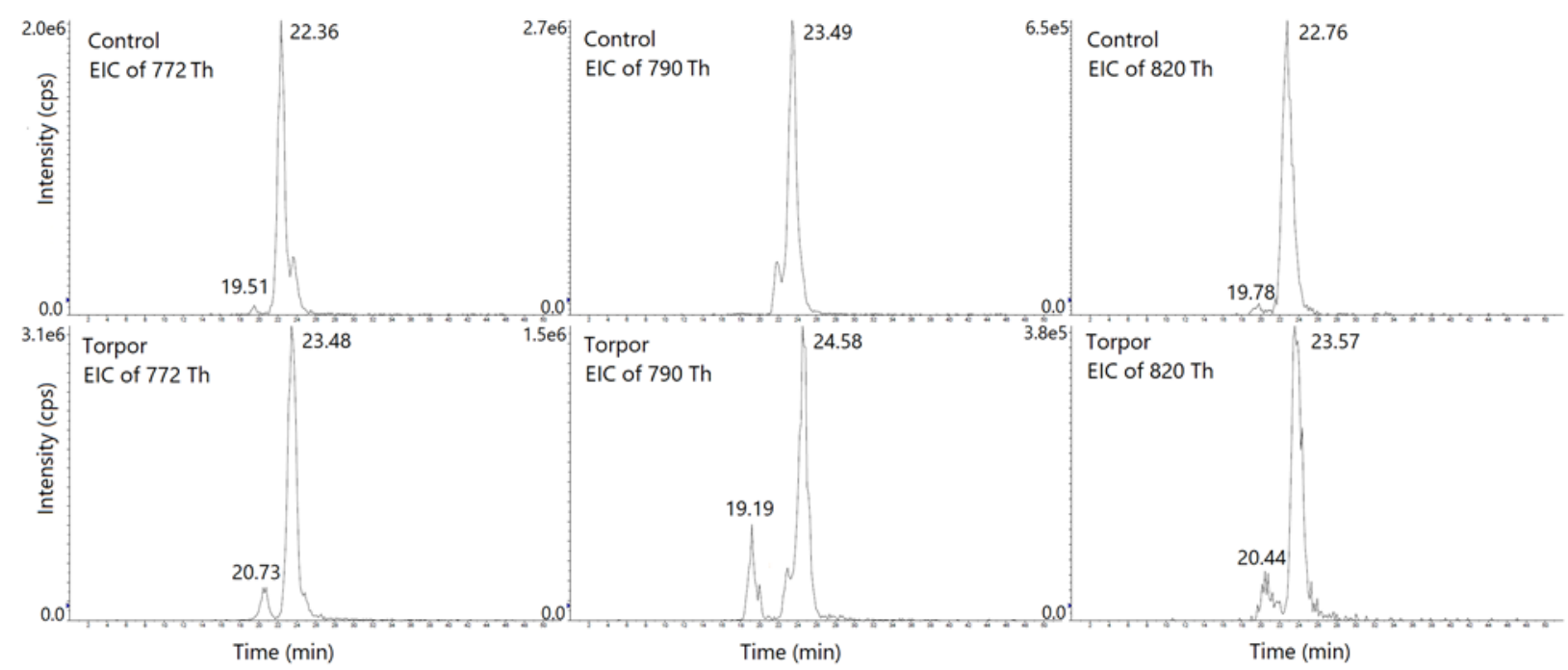

Figure 3:3. The six PC chromatograms were obtained using PIS of 184 Th. The top three spectra are representative EICs from control samples, while the bottom three spectra are representatives of torpor samples. The left column portrays EICs of $772 \mathrm{Th}$; the middle column shows EICs of $790 \mathrm{Th}$, and the right column portrays EICs of $820 \mathrm{Th}$. In all three examples, the earlier eluting peaks are significantly upregulated during torpor. Two different brand new in-house columns were used for the control and torpor triplicates. Thus there was an Rt shift that can be observed in the spectra. Using the Rt of the internal standard, corrected retention times (cRts) for all phospholipids were calculated. The average cRt for both control 772 Th peaks are 9.0 and $11.8 \mathrm{~min}$, while the torpor counterparts were 9.0 min and 11.9 min. The average cRt for both control 790 Th peaks are 7.6 and $12.9 \mathrm{~min}$; the torpor counterparts were $7.6 \mathrm{~min}$ and $12.9 \mathrm{~min}$. The average cRt for both control 820 Th peaks are 9.3 and $12.2 \mathrm{~min}$; the torpor counterparts were $8.9 \mathrm{~min}$ and $12.2 \mathrm{~min}$.

It is important to note that most of the 28 significantly upregulated PCs are low abundance lipids. The only three exceptions are 732,756 , and $774 \mathrm{Th}$. The peak at 756 Th has a torpor percent standardized average area of $12.1 \%$ in comparison to the biggest PC average area (PC 756 Th). The torpor standardized average area of 732 Th is 8.56 $\%$ and of 774 Th is $4.39 \%$ in comparison to PC 756 Th. The next highest abundance PC peak is 770 Th and has a standardized average area of $1.49 \%$, while the smallest 
abundance peak is 728 Th with a $0.05 \%$ average area value in comparison to PC 756 Th. Excluding the three most abundant significant PCs, calculating the percent for the average of all 25 standardized average areas yields an average of $0.62 \%$ abundance (in comparison to the most abundant PC 756 Th). These calculations used the torpor values, which are much higher than the control values. These calculations reveal that mostly low abundance PC species are being upregulated during torpor, suggesting a tightly regulated molecular mechanism for the surprisingly moderate change of the phospholipidome during hibernation.

With the PIS of $184 \mathrm{Th}$, and after careful correction of PC M+1 isotopic contamination, a total of 8 SM species were found in the torpor and control samples, all above the LoQ. After statistical analysis and $\mathrm{BH}$ correction, a total of $2 \mathrm{SMs}$ were found to be statistically significant (Table 3:1). The statistically insignificant peaks are presented in Table S. 3:2. The two statistically significant SM peaks had $\mathrm{m} / \mathrm{z}$ of 701 and 729 , and experienced a 2.71 and 2.91 -fold increase, respectively, during torpor. These two significant SM peaks did not have earlier elution profiles in comparison to other SMs with similar masses (703 Th and 731 Th peaks).

The NL scan of $141 \mathrm{Da}$ enabled the identification of $27 \mathrm{PE}$ species in the torpor samples, all having values above their LoQ. By contrast, the control samples identified the same 25 PE species, while one of the peaks identified in torpor was BLoQ and the other was BLoD. After statistical analysis and BH correction, only the earlier eluting PE peak with $\mathrm{m} / \mathrm{z}$ of 758 and an average cRt of $10.2 \mathrm{~min}$ in torpor samples was found to be significant (Figure 3:2B). The 758 Th counterpart was BLoD in the control samples, and therefore this became the only phospholipid to appear during torpor fully. It is important 
to note that both the statistically significant PE with an earlier elution time and the other non-significant 758 Th PE, were above yet close to the LoQ threshold. The statistically insignificant PE peaks are presented in Table S. 3:3. The statistically significant PE did have a significantly earlier elution time in comparison to all the other PE peaks. Only one PE peak out of 27 was statistically increased during torpor, suggesting that alterations to the PE lipidome are either strictly regulated or not a priority during hibernation.

The NL scan of $185 \mathrm{Da}$ enabled the identification of $7 \mathrm{PS}$ species in the torpor samples, all with values above their LoQ (despite several peaks being close the to the LoQ threshold). The control samples identified 6 PS species, the PS with $\mathrm{m} / \mathrm{z}$ of 834 was BLoQ in the control replicates. After statistical analysis and BH correction, the only PS peak to be statistically significant was 834 Th PS with an average cRt of 11.0 in control replicates and 11.1 in the torpor samples (Table 3:1). The statistically insignificant PS peaks are presented in Table S. 3:4. There are fewer PS species in comparison to PE and PC species. PS species in general also have lower concentrations than their PE, PC or SM counterparts in the liver tissue. Alterations to the PS lipidome do not seem to be a priority during hibernation, or it is strictly regulated.

\subsubsection{Q-TOF structural identification using high-resolution $\mathrm{m} / \mathrm{z}$ values}

The tandem MS experiments performed on the triple quadrupole linear ion trap MS were useful for quantitation and polar head group identification. To further elucidate the structure of the statistically significant phospholipids, positive ESI MS and negative ESI MS experiments were carried out using a Q-TOF instrument. The produced MS spectra 
are more complex than the PIS and NL scans previously produced. This is because the high-resolution MS spectra do not filter any lipid classes. Instead every single lipid category, class and subclass present in the extract will be present in the positive or negative ESI spectra depending which mode is analyzed at the time. However, having a list of statistically significant phospholipids allowed for a targeted structural identification approach. The positive ESI high-resolution masses of all statistically significant species were used to calculate the total number of carbons and unsaturations on their combined radyl groups (Table 3:2). The high-resolution masses produced by negative ESI MS experiments values were used to confirm the structural information provided by the positive ESI spectra (Table 3:2). During negative ESI experiments, deprotonated PC acetate adducts, deprotonated PE species, and deprotonated PS are produced.

Table 3:2. High-resolution $m / z$ values obtained via positive ESI and negative ESI using a $Q-T O F$ instrument.

\begin{tabular}{|c|c|c|c|c|c|c|}
\hline \multicolumn{3}{|c|}{ Positive ESI [M+H] $^{+}$} & \multicolumn{3}{|c|}{ Negative ESI $[\mathrm{M}+\mathrm{OAc}]^{-} /[\mathrm{M}-\mathrm{H}]^{-}$} & \multirow{2}{*}{ Structure } \\
\hline $\begin{array}{c}\text { Theoretical } \\
\mathbf{m} / \mathbf{z}\end{array}$ & $\begin{array}{c}\text { Experimental } \\
\mathrm{m} / \mathrm{z}\end{array}$ & Delta & $\begin{array}{c}\text { Theoretical } \\
\mathbf{m} / \mathbf{z}\end{array}$ & $\begin{array}{c}\text { Experimental } \\
\mathrm{m} / \mathbf{z}\end{array}$ & Delta & \\
\hline 676.4912 & 676.4924 & -0.0012 & 734.4967 & 734.4974 & -0.0007 & $\operatorname{PC}(28: 1)$ \\
\hline 728.5225 & 728.5265 & -0.0040 & 786.5280 & 786.5268 & 0.0012 & PC(32:3) \\
\hline 732.5538 & 732.5550 & -0.0012 & 790.5593 & 790.5604 & -0.0011 & PC(32:1) \\
\hline 744.5538 & 744.5537 & 0.0001 & 802.5593 & 802.5597 & -0.0004 & PC(33:2) \\
\hline 754.5381 & 754.5380 & 0.0001 & 812.5436 & 812.5455 & -0.0019 & PC(34:4) \\
\hline 756.5538 & 756.5548 & -0.0010 & 814.5593 & 814.5606 & -0.0013 & PC(34:3) \\
\hline 766.5381 & 766.5392 & -0.0011 & 824.5436 & 824.5443 & -0.0007 & PC(35:5) \\
\hline 770.5694 & 770.5686 & 0.0008 & 828.5749 & 828.5750 & -0.0001 & PC(35:3) \\
\hline 772.4917 & 772.4939 & -0.0022 & 830.4972 & 830.4971 & 0.0001 & PC(36:9) \\
\hline 774.5068 & 774.5075 & -0.0007 & 832.5123 & 832.5131 & -0.0008 & PC(36:8) \\
\hline 774.6007 & 774.5999 & 0.0008 & 832.6062 & 832.6056 & 0.0006 & $P C(35: 1)$ \\
\hline 788.5230 & 788.5228 & 0.0002 & 846.5285 & 846.5306 & -0.0021 & PC(37:8) \\
\hline 790.5381 & 790.5398 & -0.0017 & 848.5436 & 848.5427 & 0.0009 & PC(37:7) \\
\hline 798.6007 & 798.6008 & -0.0001 & 856.6062 & 856.6084 & -0.0022 & PC(37:3) \\
\hline
\end{tabular}




\begin{tabular}{|c|c|c|c|c|c|c|}
\hline 800.5225 & 800.5234 & -0.0009 & 858.5280 & 858.5302 & -0.0022 & PC(38:9) \\
\hline 802.5381 & 802.5374 & 0.0007 & 860.5436 & 860.5415 & 0.0021 & PC(38:8) \\
\hline 814.5387 & 814.5402 & -0.0015 & 872.5442 & 872.5437 & 0.0005 & PC(39:9) \\
\hline 816.5907 & 816.5886 & 0.0021 & 874.5962 & 874.5989 & -0.0027 & PC(39:8) \\
\hline 818.5711 & 818.5711 & 0.0000 & 876.5766 & 876.5772 & -0.0006 & PC(39:7) \\
\hline 820.5851 & 820.5871 & -0.0020 & 878.5906 & 878.5912 & -0.0006 & PC(39:6) \\
\hline 824.6528 & 824.6582 & -0.0054 & 882.6583 & 882.6597 & -0.0014 & PC(0-40:4) \\
\hline 826.6684 & 826.6723 & -0.0039 & 884.6739 & 884.6741 & -0.0002 & PC(0-40:3) \\
\hline 828.6841 & 828.6830 & 0.0011 & 886.6896 & 886.6924 & -0.0028 & PC(0-40:2) \\
\hline 830.5694 & 830.5697 & -0.0003 & 888.5749 & 888.5763 & -0.0014 & PC(40:8) \\
\hline 840.6477 & 840.6471 & 0.0006 & 898.6532 & 898.6552 & -0.0020 & PC(40:3) \\
\hline 846.6377 & 846.6380 & -0.0003 & 904.6431 & 904.6439 & -0.0008 & PC(0-42:7) \\
\hline 848.6528 & 848.6541 & -0.0013 & 906.6583 & 906.6598 & -0.0015 & PC(0-42:6) \\
\hline 850.6684 & 850.6728 & -0.0044 & 908.6739 & 908.6749 & -0.0010 & PC(41:5) \\
\hline \multicolumn{7}{|c|}{ Sphingomyelin } \\
\hline 701.5591 & 701.5593 & -0.0002 & NA & NA & NA & SM(d34:2) \\
\hline 729.5905 & 729.5915 & -0.0010 & NA & NA & NA & SM(d36:2) \\
\hline \multicolumn{7}{|c|}{ Phosphatidylethanolamine } \\
\hline 758.4755 & 758.4774 & -0.0019 & 756.4599 & 756.4597 & 0.0002 & PE(38:9) \\
\hline \multicolumn{7}{|c|}{ Phosphatidylserine } \\
\hline 834.5280 & 834.5301 & -0.0021 & 832.5124 & 832.5131 & -0.0008 & PS(40:7) \\
\hline
\end{tabular}

Out of the 28 upregulated PC species, 14 of them had 6 or more unsaturations. From the 11 upregulated early elution time PCs identified in the previous section, 10 of them were confirmed to have more than 6 unsaturations with exact mass data, thus explaining their early elution profiles. The two most abundant significantly changing PCs were $\mathrm{PC}(32: 1)$ and $\mathrm{PC}(34: 3)$. The third most abundant peak was the polyunsaturated PC(36:8). The high amount of upregulated polyunsaturated PCs indicates a precise biological mechanism to maintain cellular function during torpor by increasing the fluidity of cellular membranes via polyunsaturated PCs.

Surprisingly, out of the 28 significant PC peaks, 12 were PCs that had an odd number of carbons on their radyl moieties. These peaks were: PC (33:2), PC(35:5), 
PC(35:3), PC(35:1), PC(37:8), PC(37:7), PC(37:3), PC(39:9), PC(39:8), PC(39:7), PC(39:6) and PC(41:5). This is a surprising amount of odd chained PC peaks found upregulated during hibernation. Out of these 12 odd radyl PCs, 6 of them possess 6 or more unsaturations. The odd composition of radyl species could be essential in reducing packing density of lipid membranes, thus increasing membrane fluidity to counteract the effects of much lower body temperature. Out of the 28 significant PCs, the one with the smallest average cRt is $\mathrm{PC}(37: 7)$ [790.5398 Th] with an average cRt of $7.6 \mathrm{~min}$ in both control and torpor samples. PC (28:1) [676.4924 Th] has an average cRt of $7.9 \mathrm{~min}$ on the control samples and 8.0 min on the torpor samples. Despite $\operatorname{PC}(28: 1)$ having 9 carbons less on their radyl groups than $\mathrm{PC}(37: 7), \mathrm{PC}(37: 7)$ manages to elute earlier by being more hydrophilic due to the multiple unsaturations, in addition to interacting less strongly with the even chained hydrophobic stationary phase due to being a bit shorter than PC(28:1) (hydrophobicity increases with increasing chain length). Phospholipid species with shorter elution times are more hydrophilic and will increase membrane fluidity by disrupting packing density.

Despite all significant odd chained PC species being present in low concentrations (small standardized average areas), their odd chain and a high degree of unsaturation could be indispensable in controlling and maintaining membrane fluidity.

The 701.5593 Th SM peak has a predicted structure of SM(d34:2) and the 729.5915 Th SM peak has a predicted structure of SM(d36:2). It is still unclear the reason for the upregulation of these two SM species during torpor. The structural information of the statistically significant PE and PS peaks were PE(38:9) and PS(40:7). Both of these phospholipids are highly unsaturated with 9 and 7 unsaturation events respectively. 
Despite a single PE and a single PS species being upregulated during torpor, the upregulated phospholipids are highly unsaturated phospholipids that will increase membrane fluidity. PS and PE species are mostly present in the inner leaflet of the plasma membrane and are involved in providing curvature to the plasma membrane ${ }^{24,25}$. Perhaps due to this important role, torpor mechanisms in PE and PS species are more conservative and only target a subsection of the PE and PS lipidomes.

\subsubsection{Fatty acid chain identification via negative ESI MS/MS experiments}

To obtain further structural information of the statistically significant upregulated phospholipids, negative ESI product ion experiments of [PC+OAc] $]^{-}[\mathrm{PE}-\mathrm{H}]^{-}$and $[\mathrm{PS}-\mathrm{H}]^{-}$ were conducted. In conjunction with the total number of carbons and unsaturations obtained from the high-resolution masses, these experiments allowed to elucidate the number of carbons and unsaturations specific to each radyl moiety. Since most of the significant phospholipids have low concentrations, the exact masses of experimental radyl peaks served to distinguish real fatty acid peaks from noise. In order to properly elucidate the structure of a diacyl phospholipid, the complementary fatty acid peak pairs in addition to appropriate accurate masses for both had to be identified. The more intense fatty acid peak was reported first with the less abundant complementary pair reported under it. The main phospholipid structure was reported using the fatty acyl pair with higher intensity, while the secondary structure was calculated using the second most intense fatty acyl pair. All the calculated structures are presented in Table 3:3. We did not attempt to assign each radyl group to the $s n-1$ or $s n-2$ position since the high number of unsaturations and their position greatly affect radyl dissociation after CID, thus 
substantially affecting the fatty acid signal. Since O-alkyl or O-alkyl-1'-enyl residues do not produce ions after CID, the O-alkyl or O-alkyl-1'-enyl chain length was deduced from the single fatty acid peak encountered. Since many of the statistically significant peaks were low in abundance, only the peak structures that were confidently identified were included in Table 3:3.

Table 3:3. Radyl information for the primary and secondary $P C, P E$ and $P S$ species obtained from negative ESI MS/MS experiments using a Q-TOF instrument.

\begin{tabular}{|c|c|c|c|c|c|c|c|c|c|}
\hline \multirow{2}{*}{$\begin{array}{l}\text { Positive } \\
\text { ESI } \\
\mathrm{m} / \mathrm{z}\end{array}$} & \multirow{2}{*}{ Lipid Structure } & \multicolumn{4}{|c|}{ Predominant Structure } & \multicolumn{4}{|c|}{ Secondary Structure } \\
\hline & & $\mathrm{C} \#: \mathrm{X}$ & THEO & EXP & Delta & C\#:X & THEO & EXP & Delta \\
\hline \multicolumn{10}{|c|}{ Phosphatidylcholine } \\
\hline \multirow[t]{2}{*}{676} & \multirow[t]{2}{*}{$\mathrm{PC}(16: 0 / 12: 1)$} & C16:0 & 255.2324 & 255.2293 & 0.0031 & & & & \\
\hline & & C12:1 & 197.1542 & 197.1501 & 0.0041 & & & & \\
\hline \multirow[t]{2}{*}{728} & \multirow[t]{2}{*}{$\mathrm{PC}(18: 3 / 14: 0)$} & C14:0 & 227.2011 & 227.2000 & 0.0011 & & & & \\
\hline & & C18:3 & 277.2168 & 277.2182 & -0.0014 & & & & \\
\hline \multirow[t]{2}{*}{732} & \multirow[t]{2}{*}{$\mathrm{PC}(16: 1 / 16: 0)$} & C16:1 & 253.2168 & 253.2170 & -0.0002 & & & & \\
\hline & & C16:0 & 255.2324 & 255.2329 & -0.0005 & & & & \\
\hline \multirow[t]{2}{*}{744} & \multirow{2}{*}{$\begin{array}{l}\text { 1-PC(18:2/15:0) } \\
2-P C(16: 0 / 17: 2)\end{array}$} & C18:2 & 279.2324 & 279.2290 & 0.0034 & C16:0 & 255.2324 & 255.2293 & 0.0031 \\
\hline & & C15:0 & 241.2168 & 241.2144 & 0.0024 & C17:2 & 265.2168 & 265.2127 & 0.0041 \\
\hline \multirow[t]{2}{*}{754} & \multirow{2}{*}{$\begin{array}{l}\text { 1-PC(18:3/16:1) } \\
2-P C(18: 2 / 16: 2)\end{array}$} & C18:3 & 277.2168 & 277.2177 & -0.0009 & C18:2 & 279.2324 & 279.2331 & -0.0007 \\
\hline & & C16:1 & 253.2168 & 253.2174 & -0.0006 & C16:2 & 251.2011 & 251.1997 & 0.0014 \\
\hline \multirow[t]{2}{*}{756} & \multirow{2}{*}{$\begin{array}{l}\text { 1-PC(18:2/16:1)\# } \\
2-P C(18: 3 / 16: 0)\end{array}$} & C18:2 & 279.2324 & 279.2302 & 0.0022 & C18:3 & 277.2168 & 277.2145 & 0.0023 \\
\hline & & C16:1 & 253.2168 & 253.2149 & 0.0019 & C16:0 & 255.2324 & 255.2308 & 0.0016 \\
\hline \multirow[t]{2}{*}{770} & \multirow{2}{*}{$\begin{array}{l}\text { 1-PC(18:2/17:1)\# } \\
\text { 2-PC(18:1/17:2) }\end{array}$} & C18:2 & 279.2324 & 279.2334 & -0.0010 & C18:1 & 281.2481 & 281.2489 & -0.0008 \\
\hline & & C17:1 & 267.2324 & 267.2294 & 0.0030 & C17:2 & 265.2168 & 265.2152 & 0.0016 \\
\hline \multirow[t]{2}{*}{774} & \multirow{2}{*}{$\begin{array}{l}\text { 1-PC(18:1/17:0) } \\
2-P C(18: 0 / 17: 1)\end{array}$} & C18:1 & 281.2481 & 281.2467 & 0.0014 & C18:0 & 283.2637 & 283.2606 & 0.0031 \\
\hline & & C17:0 & 269.2481 & 269.2461 & 0.0020 & C17:1 & 267.2324 & 267.2300 & 0.0024 \\
\hline \multirow[t]{2}{*}{798} & \multirow{2}{*}{$\begin{array}{l}\text { 1-PC(20:3/17:0) } \\
2-P C(19: 1 / 18: 2)\end{array}$} & C20:3 & 305.2481 & 305.2405 & 0.0076 & C19:1 & 295.2637 & 295.2680 & -0.0043 \\
\hline & & C17:0 & 269.2481 & 269.2393 & 0.0088 & C18:2 & 279.2324 & 279.2290 & 0.0034 \\
\hline \multirow[t]{2}{*}{818} & \multirow[t]{2}{*}{$\mathrm{PC}(18: 1 / 21: 6)$} & C18:1 & 281.2481 & 281.2478 & 0.0003 & & & & \\
\hline & & C21:6 & 313.2168 & 313.2176 & -0.0008 & & & & \\
\hline 824 & $\begin{array}{l}\text { 1-PC(0-24:4/16:0) } \\
2-P C(0-22: 3 / 18: 1)\end{array}$ & C16:0 & 255.2324 & 255.2314 & 0.0010 & C18:1 & 281.2481 & 281.2436 & 0.0045 \\
\hline 826 & $\begin{array}{l}\text { 1-PC(O-24:3/16:0) } \\
\text { 2-PC(O-22:2/18:1) }\end{array}$ & C16:0 & 255.2324 & 255.2325 & -0.0001 & C18:1 & 281.2481 & 281.2495 & -0.0014 \\
\hline 828 & $\begin{array}{l}\text { 1-PC(O-24:2/16:0) } \\
\text { 2-PC(O-22:1/18:1) }\end{array}$ & C16:0 & 255.2324 & 255.2310 & 0.0014 & C18:1 & 281.2481 & 281.2439 & 0.0042 \\
\hline \multirow[t]{2}{*}{830} & \multirow{2}{*}{$\begin{array}{l}\text { 1-PC(18:2/22:6) } \\
2-P C(20: 4 / 20: 4)\end{array}$} & C18:2 & 279.2324 & 279.2333 & -0.0009 & C20:4 & 303.2324 & 303.2311 & 0.0013 \\
\hline & & C22:6 & 327.2324 & 327.2327 & -0.0003 & C20:4 & 303.2324 & 303.2311 & 0.0013 \\
\hline
\end{tabular}




\begin{tabular}{|c|c|c|c|c|c|c|c|c|c|}
\hline 846 & $\begin{array}{l}\text { 1-PC(0-24:6/18:1) } \\
2-P C(0-24: 7 / 18: 0)\end{array}$ & C18:1 & 281.2480 & 281.2438 & 0.0043 & C18:0 & 283.2637 & 283.2637 & 0.0000 \\
\hline \multicolumn{10}{|c|}{ Phosphatidylethanolamine } \\
\hline \multirow[t]{2}{*}{758} & \multirow[t]{2}{*}{ PE(18:2/20:7) } & C18:2 & 279.2324 & 279.2395 & -0.0605 & & & & \\
\hline & & C20:7 & 297.1855 & 297.1915 & -0.0071 & & & & \\
\hline \multicolumn{10}{|c|}{ Phosphatidylserine } \\
\hline \multirow[t]{2}{*}{834} & \multirow[t]{2}{*}{ PS(18:1/22:6) } & C18:1 & 281.2481 & 281.2402 & 0.0079 & & & & \\
\hline & & C22:6 & 327.2324 & 327.2301 & 0.0023 & & & & \\
\hline
\end{tabular}

\# - Indicates that the labeled structure was the predominant structure despite secondary structures found with much smaller intensities.

Odd chain radyl groups have been widely assumed to be absent in mammalian cells and tissues. The radyl data obtained from the negative ESI MS/MS experiments further cements the previously assigned odd chained PCs by demonstrating complementary odd and even chained fatty acids in their respective product ion spectra. Figure 3:4 demonstrates the negative ESI MS/MS spectra for three odd chained PC representatives. The different odd radyl moieties found were C15:0 [PC(18:2/15:0)], C17:2 $\quad[P C(16: 0 / 17: 2)], \quad C 17: 1 \quad[P C(18: 2 / 17: 1)], \quad C 17: 0 \quad[P C(18: 1 / 17: 0)], \quad C 19: 1$ [PC(19:1/18:2)] and C21:6 [PC(18:1/21:6].

Unfortunately, many of the polyunsaturated (GPLs with 6 or more unsaturations) PCs were low in abundance and did not produce confident spectra to identify the distribution of unsaturation between the two radyl groups. The 7 (bolded in Figure 3:3) different polyunsaturated phospholipids whose radyl groups were successfully identified are the following: $\mathrm{PC}(18: 1 / 21: 6), \mathrm{PC}(18: 2 / 22: 6), \mathrm{PC}(20: 4 / 20: 4), \mathrm{PC}(\mathrm{O}-24: 6 / 18: 1), \mathrm{PC}(\mathrm{O}-$ 24:7/18:0), PE(18:2/ 20:7) and PS(18:1/22:6). The PS22:6 and PE20:7 radyl groups identified are particular due to their high amount of unsaturation in a single radyl group. The 21:6 radyl group is more peculiar as this is a highly unsaturated and odd-chained fatty acid that is being significantly upregulated in a hibernating mammalian liver. The 
identification of radyl groups allowed to confirm and expand the structural information from the significant statistical phospholipids. The radyl group information also highlighted the relevance of odd chained radyl groups and highly unsaturated radyl groups to decrease membrane rigidity and maintain functional cells during cold temperatures.

There is no known literature available to compare the results obtained. The overall phospholipid distribution in the squirrel liver tissue is consistent with literature values obtained using two-dimensional thin-layer chromatography, which showed that the squirrel liver lipid bilayer consists predominantly of PC and PE lipids ${ }^{26}$. There are no studies that show that the squirrel liver tissue increases its levels of unsaturated phospholipids in adjustment to cold. Also, there is no literature on the identify of the structures of phospholipids that increase in concentration to maintain the liver lipid bilayer fluidity during the cold months. In this chapter are presented novel results which indicate that the squirrel liver lipid bilayer fluidity is preserved by increasing the concentrations of the predominantly polyunsaturated lipids in the winter as discussed earlier. 

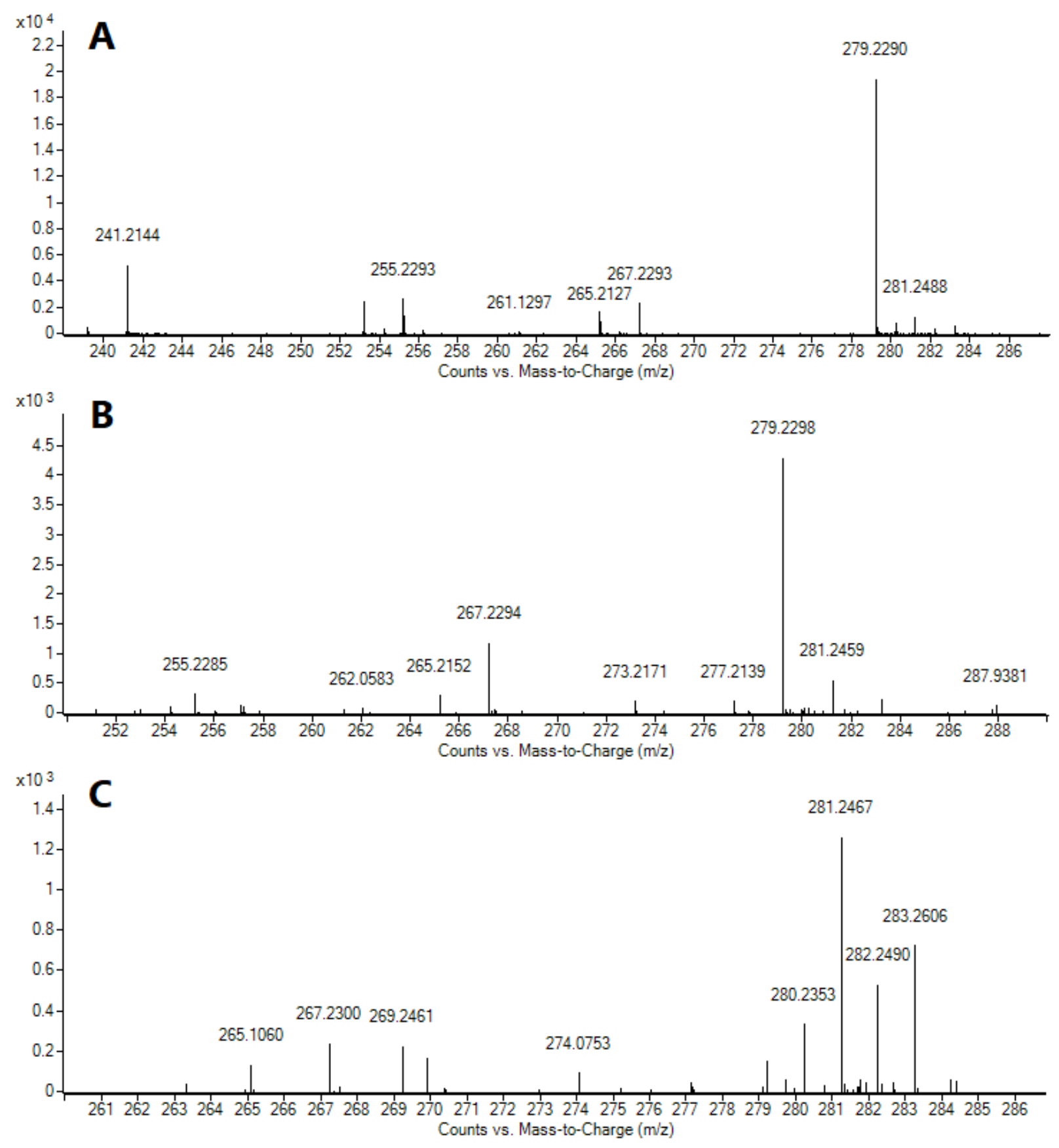

Figure 3:4. Negative ESI product ion scan spectra of three odd-chained statistically significant PC species. The product ion scan for PC (33:2) [802.5597 Th] in panel A demonstrates the presence of $279.2290 \mathrm{Th}$ (C18:2) and 241.2144 Th (C15:0), as well as 255.2293 Th (C16:0) and 265.2127 Th (C17:2). Panel B shows the product ion scan for PC (35:3) [828.5749 Th] where 279.2298 Th (C18:2) and 267.2294 Th (C17:1), as well as 281.2459 Th (C18:1) and 265.2152 Th (C17:2) are present. Panel C shows the product ion scan for PC (35:1) [832.6062 Th] where 281.2467 Th (C18:1) and 269.2461 Th (C17:0), as well as 283.2606 Th (C18:0) and 267.2300 Th (C17:1) are present. 


\subsection{Conclusion}

Vital organs, like the liver, have to remain functional during the entire hibernation period of an organism, thus investigating the phospholipidome dynamics of liver tissues provided insight into the required adaptations that preserve cellular function and viability at temperatures lower than $32^{\circ} \mathrm{C}$ from their euthermic state. This study demonstrated that phospholipids play an important role during torpor to keep the liver cells and the organ functioning. Rather than experiencing a global and untargeted increase of desaturase activity, torpor seems to target a specific subset of the phospholipidome. The highly unsaturated $\mathrm{PE}(18: 2 / 20: 7)$ is exclusively upregulated out of a total of 27 identified $\mathrm{PE} \mathrm{m/z}$ values. Similar to PEs, the only upregulated PS is the highly unsaturated PS(18:1/22:6) out of a total of 7 identified PS m/z values. SM(d34:2) and SM(d36:2) were upregulated by a factor of 2.71 and 2.91, respectively out of a total of 8 identified SMs m/z values. A total of $28 \mathrm{PC}$ species from a total of $88 \mathrm{PC} \mathrm{m} / \mathrm{z}$ values identified experienced significant upregulation. The fold increase of PC species ranged from 1.6x increase [PC(16:0/12:1)] to $23.19 x$ increase $[\mathrm{PC}(37: 8)]$.

Despite a large number of PC species being upregulated, 25 out of the 28 upregulated PCs were low abundance PCs, highlighting specific biochemical pathways that do not target whole lipid classes but specific lipids. Since no lipid was found to be statistically significantly downregulated, the phospholipidome changes do not seem to be driven by the activation of unsaturates, as their activation would lead to the increase of one phospholipid at the expense of another.

From the high-resolution data and radyl identification experiments, it became evident that odd chained PCs and polyunsaturated PCs were the primary lipids being 
upregulated during torpor. It is important to note that despite odd chained PC lipids and polyunsaturated PC lipids being increased the most during torpor, these lipids were still abundance phospholipids after the torpor upregulation. Higher concentrations of odd chain PCs along with polyunsaturated PCs seem to be integral components in increasing the cellular membrane's fluidity, allows cellular membranes and cells to remain functional even at temperatures close to $5^{\circ} \mathrm{C}$.

From the TIC obtained with the high-resolution spectrometer, it became evident that glycerolipids are the dominant lipid category present in TLS liver tissue samples. These glycerolipids could play vital roles in the upkeep and biochemical function of torpor tissues. To further understand the dynamics of hibernation this category of lipids should be explored via LC-MS in the future. Cholesterol concentration in cellular membranes is well known to regulate membrane fluidity ${ }^{27}$. Further detailed MS studies of sterol lipids during hibernation are also required.

\subsection{Supplementary information}

Table S. 3:1. Non-statistically significant PC peaks after student t-test analysis and Benjamini-Hochberg correction.

\begin{tabular}{|c|c|c|c|c|c|c|c|c|}
\hline \multirow[b]{2}{*}{$\mathrm{m} / \mathrm{z}$} & \multicolumn{3}{|c|}{ Control } & \multicolumn{3}{|c|}{ Torpor } & \multirow[b]{2}{*}{$\begin{array}{c}\mathrm{P}- \\
\text { value }\end{array}$} & \multirow[b]{2}{*}{$\begin{array}{c}\text { Fold } \\
\text { change }\end{array}$} \\
\hline & $\begin{array}{l}\text { Standardized } \\
\text { average } \\
\text { retention } \\
\text { time (min) }\end{array}$ & $\begin{array}{l}\text { Standardized } \\
\text { average area }\end{array}$ & $\begin{array}{l}\text { Standard } \\
\text { deviation }\end{array}$ & $\begin{array}{l}\text { Standardized } \\
\text { average } \\
\text { retention } \\
\text { time (min) }\end{array}$ & $\begin{array}{l}\text { Standardized } \\
\text { average area }\end{array}$ & $\begin{array}{l}\text { Standard } \\
\text { deviation }\end{array}$ & & \\
\hline 454 & 0.0 & $1.00 \mathrm{E}+00$ & $0.00 \mathrm{E}+00$ & 0.0 & $1.00 \mathrm{E}+00$ & $0.00 E+00$ & & 1.00 \\
\hline 496 & 3.8 & $4.03 E-01$ & $1.75 \mathrm{E}-01$ & 4.3 & 1.69E-01 & 5.73E-02 & 0.0929 & 0.42 \\
\hline 522 & 4.3 & $1.41 \mathrm{E}-01$ & $6.30 \mathrm{E}-02$ & 4.8 & $5.14 \mathrm{E}-02$ & $1.38 \mathrm{E}-02$ & 0.0736 & 0.36 \\
\hline 524 & 5.5 & $9.00 \mathrm{E}-01$ & $2.11 \mathrm{E}-01$ & 5.5 & $3.08 \mathrm{E}-01$ & 8.02E-02 & 0.0105 & 0.34 \\
\hline 650 & 5.1 & $1.62 \mathrm{E}-01$ & $1.36 \mathrm{E}-01$ & 5.4 & 8.61E-02 & $2.27 \mathrm{E}-02$ & 0.3961 & 0.53 \\
\hline 674 & 7.9 & $1.20 \mathrm{E}-01$ & 1.92E-02 & 8.2 & $2.98 \mathrm{E}-01$ & 5.32E-02 & 0.0055 & 2.48 \\
\hline
\end{tabular}




\begin{tabular}{|c|c|c|c|c|c|c|c|c|}
\hline 688 & 10.1 & $4.66 \mathrm{E}-02$ & $6.21 \mathrm{E}-03$ & 10.7 & $1.99 E-01$ & $8.41 \mathrm{E}-02$ & 0.0353 & 4.27 \\
\hline 690 & 10.0 & $8.43 \mathrm{E}-02$ & $2.56 \mathrm{E}-02$ & 10.8 & 3.09E-01 & $1.17 \mathrm{E}-01$ & 0.0311 & 3.66 \\
\hline 700 & 9.9 & $7.46 \mathrm{E}-02$ & 1.47E-02 & 10.3 & $2.29 \mathrm{E}-01$ & $5.42 \mathrm{E}-02$ & 0.0090 & 3.07 \\
\hline 708 & 10.5 & 4.09E-02 & 1.10E-02 & 11.2 & $2.00 \mathrm{E}-01$ & $1.28 \mathrm{E}-01$ & 0.0977 & 4.90 \\
\hline 716 & 11.1 & $1.88 \mathrm{E}-01$ & $4.21 \mathrm{E}-02$ & 11.2 & 5.92E-01 & 1.07E-01 & 0.0037 & 3.15 \\
\hline 718 & 11.0 & $3.79 \mathrm{E}-01$ & 8.33E-02 & 11.2 & $9.65 \mathrm{E}-01$ & $1.52 \mathrm{E}-01$ & 0.0042 & 2.54 \\
\hline 720 & 11.9 & $3.01 \mathrm{E}-01$ & $2.24 \mathrm{E}-02$ & 11.8 & $5.62 \mathrm{E}-01$ & $1.03 \mathrm{E}-01$ & 0.0127 & 1.87 \\
\hline 730 & 10.3 & 7.14E-01 & $9.52 \mathrm{E}-02$ & 10.5 & $1.39 E+00$ & $2.13 \mathrm{E}-01$ & 0.0075 & 1.94 \\
\hline 734 & 11.5 & $3.54 \mathrm{E}+00$ & 3.17E-01 & 11.5 & $4.28 \mathrm{E}+00$ & $1.35 E+00$ & 0.4068 & 1.21 \\
\hline 746 & 12.1 & $2.09 E+00$ & 1.93E-01 & 11.7 & $4.31 \mathrm{E}+00$ & $9.90 \mathrm{E}-01$ & 0.0189 & 2.06 \\
\hline 758 & 11.3 & $7.88 \mathrm{E}+01$ & $2.20 \mathrm{E}+00$ & 11.4 & $1.72 E+02$ & $2.90 \mathrm{E}+01$ & 0.0052 & 2.18 \\
\hline 760 & 11.9 & $5.71 \mathrm{E}+01$ & $2.86 \mathrm{E}+00$ & 12.0 & $7.85 E+01$ & $1.82 \mathrm{E}+01$ & 0.1137 & 1.38 \\
\hline 768 & 11.7 & $1.36 \mathrm{E}+00$ & $1.91 \mathrm{E}-01$ & 11.6 & $2.36 \mathrm{E}+00$ & $3.32 \mathrm{E}-01$ & 0.0108 & 1.73 \\
\hline 772 & 11.8 & $3.38 \mathrm{E}+00$ & $3.02 E-01$ & 11.9 & $1.08 \mathrm{E}+01$ & $2.63 E+00$ & 0.0083 & 3.20 \\
\hline 778 & 11.1 & 8.91E-01 & $1.52 \mathrm{E}-01$ & 11.2 & $1.49 \mathrm{E}+00$ & $2.52 \mathrm{E}-01$ & 0.0242 & 1.67 \\
\hline 780 & 11.1 & $8.71 \mathrm{E}+00$ & $1.27 \mathrm{E}+00$ & 11.2 & $1.96 \mathrm{E}+01$ & $4.19 E+00$ & 0.0128 & 2.24 \\
\hline 782 & 11.5 & $2.93 E+01$ & $3.03 E+00$ & 11.4 & $3.57 \mathrm{E}+01$ & $3.92 E+00$ & 0.0887 & 1.22 \\
\hline 784 & 11.6 & $3.59 E+01$ & $3.29 E+00$ & 11.8 & $9.03 E+01$ & $1.94 \mathrm{E}+01$ & 0.0086 & 2.52 \\
\hline 786 & 12.4 & $6.33 \mathrm{E}+01$ & $3.30 \mathrm{E}+00$ & 12.4 & $1.02 E+02$ & $2.70 \mathrm{E}+01$ & 0.0707 & 1.61 \\
\hline 788 & 12.8 & $3.02 \mathrm{E}+01$ & $2.79 E+00$ & 12.8 & $3.21 \mathrm{E}+01$ & $1.11 \mathrm{E}+01$ & 0.7871 & 1.06 \\
\hline 790 & 12.9 & $5.37 \mathrm{E}+00$ & $6.92 \mathrm{E}-01$ & 12.9 & $6.07 \mathrm{E}+00$ & $9.96 \mathrm{E}-01$ & 0.3710 & 1.13 \\
\hline 792 & 11.7 & $1.54 \mathrm{E}+00$ & 2.17E-01 & 11.7 & $2.23 E+00$ & $1.36 \mathrm{E}-01$ & 0.0096 & 1.45 \\
\hline 794 & 12.3 & $2.11 \mathrm{E}+00$ & $2.88 \mathrm{E}-01$ & 12.4 & $3.50 E+00$ & 8.94E-01 & 0.0629 & 1.66 \\
\hline 796 & 12.3 & $1.50 \mathrm{E}+00$ & $1.51 \mathrm{E}-01$ & 12.4 & $1.90 \mathrm{E}+00$ & $4.67 \mathrm{E}-01$ & 0.2273 & 1.27 \\
\hline 798 & 12.3 & $8.59 \mathrm{E}-01$ & $7.45 \mathrm{E}-02$ & 12.3 & $1.85 \mathrm{E}+00$ & $2.89 \mathrm{E}-01$ & 0.0046 & 2.15 \\
\hline 800 & 13.5 & $2.14 \mathrm{E}+00$ & 2.83E-01 & 12.6 & $4.32 E+00$ & $6.51 \mathrm{E}-01$ & 0.0060 & 2.02 \\
\hline 802 & 14.0 & $3.02 \mathrm{E}+00$ & $4.82 \mathrm{E}-01$ & 14.0 & $4.86 \mathrm{E}+00$ & $4.70 \mathrm{E}-01$ & 0.0092 & 1.61 \\
\hline 804 & 10.9 & $1.86 \mathrm{E}+00$ & $3.86 \mathrm{E}-01$ & 11.1 & $2.77 \mathrm{E}+00$ & $2.38 \mathrm{E}-01$ & 0.0260 & 1.49 \\
\hline 804 & 14.1 & $3.30 \mathrm{E}-01$ & $6.26 \mathrm{E}-02$ & 14.2 & $3.38 \mathrm{E}-01$ & $9.73 \mathrm{E}-02$ & 0.9153 & 1.02 \\
\hline 806 & 11.5 & $4.09 \mathrm{E}+01$ & $3.98 \mathrm{E}+00$ & 11.6 & $3.44 \mathrm{E}+01$ & $5.49 \mathrm{E}+00$ & 0.1738 & 0.84 \\
\hline 808 & 11.8 & $2.02 \mathrm{E}+01$ & $2.34 \mathrm{E}+00$ & 12.0 & $2.26 \mathrm{E}+01$ & $6.70 \mathrm{E}+00$ & 0.5943 & 1.12 \\
\hline 810 & 12.6 & $1.71 \mathrm{E}+01$ & $1.60 \mathrm{E}+00$ & 12.7 & $1.01 \mathrm{E}+01$ & $2.78 \mathrm{E}+00$ & 0.0192 & 0.59 \\
\hline 812 & 12.7 & $7.08 \mathrm{E}+00$ & 8.18E-01 & 12.8 & $4.45 E+00$ & $1.81 \mathrm{E}+00$ & 0.0828 & 0.63 \\
\hline 818 & 12.4 & $5.30 \mathrm{E}-01$ & $3.39 \mathrm{E}-02$ & 12.6 & $1.04 \mathrm{E}+00$ & $3.13 \mathrm{E}-01$ & 0.0476 & 1.97 \\
\hline 818 & 14.5 & $3.81 \mathrm{E}-01$ & 8.05E-03 & 14.6 & 8.67E-01 & $3.47 \mathrm{E}-01$ & 0.0727 & 2.27 \\
\hline 820 & 12.2 & 7.10E-01 & $7.60 \mathrm{E}-02$ & 12.2 & 9.03E-01 & $2.21 \mathrm{E}-01$ & 0.2259 & 1.27 \\
\hline 822 & 12.3 & $2.81 \mathrm{E}-01$ & $2.95 \mathrm{E}-02$ & 12.3 & $4.72 \mathrm{E}-01$ & $1.83 \mathrm{E}-01$ & 0.1495 & 1.68 \\
\hline 830 & 14.9 & $2.08 \mathrm{E}-01$ & 4.06E-02 & 15.1 & 3.87E-01 & $8.25 \mathrm{E}-02$ & 0.0282 & 1.86 \\
\hline 832 & 11.8 & $6.61 \mathrm{E}+00$ & 7.35E-01 & 11.9 & $8.02 \mathrm{E}+00$ & $2.16 \mathrm{E}+00$ & 0.3466 & 1.21 \\
\hline 834 & 12.6 & $2.86 \mathrm{E}+01$ & $2.59 \mathrm{E}+00$ & 12.7 & $2.02 \mathrm{E}+01$ & $5.08 \mathrm{E}+00$ & 0.0645 & 0.71 \\
\hline 842 & 13.6 & $6.31 \mathrm{E}-02$ & $8.52 \mathrm{E}-03$ & 11.9 & $1.79 \mathrm{E}-01$ & $5.84 \mathrm{E}-02$ & 0.0271 & 2.84 \\
\hline
\end{tabular}




\begin{tabular}{|l|l|l|l|l|l|l|l|l|}
\hline 844 & 12.6 & $4.11 \mathrm{E}-02$ & $1.24 \mathrm{E}-02$ & 12.7 & $9.84 \mathrm{E}-02$ & $2.93 \mathrm{E}-02$ & 0.0355 & 2.40 \\
\hline 860 & 12.7 & $3.13 \mathrm{E}-01$ & $6.03 \mathrm{E}-02$ & 12.6 & $2.73 \mathrm{E}-01$ & $5.05 \mathrm{E}-02$ & 0.4336 & 0.87 \\
\hline 862 & 13.0 & $2.29 \mathrm{E}-01$ & $3.25 \mathrm{E}-02$ & 13.1 & $2.75 \mathrm{E}-01$ & $5.89 \mathrm{E}-02$ & 0.2987 & 1.20 \\
\hline 864 & 13.4 & $2.39 \mathrm{E}-01$ & $4.65 \mathrm{E}-02$ & 13.5 & $3.77 \mathrm{E}-01$ & $4.36 \mathrm{E}-02$ & 0.0197 & 1.58 \\
\hline 918 & 11.4 & $4.50 \mathrm{E}-02$ & $5.05 \mathrm{E}-03$ & 11.2 & $1.87 \mathrm{E}-01$ & $7.60 \mathrm{E}-02$ & 0.0317 & 4.17 \\
\hline 920 & 15.4 & $6.69 \mathrm{E}-02$ & $1.50 \mathrm{E}-02$ & 15.4 & $1.19 \mathrm{E}-01$ & $2.42 \mathrm{E}-02$ & 0.0336 & 1.78 \\
\hline 932 & 11.3 & $5.71 \mathrm{E}-02$ & $8.70 \mathrm{E}-03$ & 11.4 & $2.79 \mathrm{E}-01$ & $1.14 \mathrm{E}-01$ & 0.0281 & 4.89 \\
\hline 934 & 11.9 & $2.92 \mathrm{E}-02$ & $4.28 \mathrm{E}-03$ & 11.9 & $1.01 \mathrm{E}-01$ & $3.28 \mathrm{E}-02$ & 0.0202 & 3.44 \\
\hline 944 & 11.9 & $2.79 \mathrm{E}-02$ & $7.30 \mathrm{E}-03$ & 12.0 & $1.49 \mathrm{E}-01$ & $5.35 \mathrm{E}-02$ & 0.0179 & 5.33 \\
\hline 952 & 11.1 & $8.64 \mathrm{E}-02$ & $9.95 \mathrm{E}-03$ & 11.4 & $2.19 \mathrm{E}-01$ & $6.27 \mathrm{E}-02$ & 0.0221 & 2.54 \\
\hline 954 & 11.9 & $5.47 \mathrm{E}-02$ & $5.33 \mathrm{E}-04$ & 11.7 & $1.33 \mathrm{E}-01$ & $3.67 \mathrm{E}-02$ & 0.0212 & 2.42 \\
\hline 958 & 12.4 & $2.15 \mathrm{E}-02$ & $7.04 \mathrm{E}-03$ & 11.9 & $1.36 \mathrm{E}-01$ & $4.45 \mathrm{E}-02$ & 0.0118 & 6.30 \\
\hline 960 & 12.4 & $3.57 \mathrm{E}-02$ & $4.65 \mathrm{E}-03$ & 12.5 & $1.65 \mathrm{E}-01$ & $6.27 \mathrm{E}-02$ & 0.0238 & 4.61 \\
\hline 980 & 12.3 & $6.74 \mathrm{E}-02$ & $7.16 \mathrm{E}-03$ & 12.4 & $1.69 \mathrm{E}-01$ & $5.84 \mathrm{E}-02$ & 0.0401 & 2.51 \\
\hline
\end{tabular}

Table S. 3:2. Non-statistically significant SM peaks after student t-test analysis and Benjamini-Hochberg correction.

\begin{tabular}{|c|c|c|c|c|c|c|c|c|}
\hline \multirow[b]{2}{*}{$\mathrm{m} / \mathrm{z}$} & \multicolumn{3}{|c|}{ Control } & \multicolumn{3}{|c|}{ Torpor } & \multirow[b]{2}{*}{$\begin{array}{c}\text { P- } \\
\text { value }\end{array}$} & \multirow[b]{2}{*}{$\begin{array}{c}\text { Fold } \\
\text { change }\end{array}$} \\
\hline & $\begin{array}{l}\text { Standardized } \\
\text { average } \\
\text { retention } \\
\text { time (min) } \\
\end{array}$ & $\begin{array}{l}\text { Standardized } \\
\text { average area }\end{array}$ & $\begin{array}{l}\text { Standard } \\
\text { deviation }\end{array}$ & $\begin{array}{l}\text { Standardized } \\
\text { average } \\
\text { retention } \\
\text { time }(\mathrm{min}) \\
\end{array}$ & $\begin{array}{l}\text { Standardized } \\
\text { average area }\end{array}$ & $\begin{array}{l}\text { Standard } \\
\text { deviation }\end{array}$ & & \\
\hline 703 & 10.6 & $3.48 \mathrm{E}+00$ & 7.77E-01 & 10.7 & $5.03 E+00$ & $9.62 \mathrm{E}-01$ & 0.0958 & 1.45 \\
\hline 731 & 11.5 & $2.78 \mathrm{E}+00$ & 3.01E-01 & 11.7 & $5.38 \mathrm{E}+00$ & $1.23 \mathrm{E}+00$ & 0.0235 & 1.93 \\
\hline 813 & 13.4 & $3.24 \mathrm{E}+00$ & $5.46 \mathrm{E}-01$ & 13.7 & $5.75 E+00$ & $2.65 \mathrm{E}+00$ & 0.1826 & 1.78 \\
\hline 815 & 14.5 & $3.58 \mathrm{E}+00$ & 7.23E-01 & 14.7 & $5.87 \mathrm{E}+00$ & $1.69 E+00$ & 0.0970 & 1.64 \\
\hline 841 & 14.3 & 5.27E-02 & $3.66 \mathrm{E}-03$ & 13.3 & $9.93 \mathrm{E}-02$ & $3.36 \mathrm{E}-02$ & 0.0753 & 1.88 \\
\hline 843 & 14.1 & $7.28 \mathrm{E}-02$ & $2.60 \mathrm{E}-02$ & 14.5 & $7.25 \mathrm{E}-02$ & $7.84 \mathrm{E}-02$ & 0.9954 & 1.00 \\
\hline
\end{tabular}

Table S. 3:3. Non-statistically significant PE peaks after student t-test analysis and Benjamini-Hochberg correction.

\begin{tabular}{|c|c|c|c|c|c|c|c|c|}
\hline \multirow[b]{2}{*}{$\mathrm{m} / \mathrm{z}$} & \multicolumn{3}{|c|}{ Control } & \multicolumn{3}{|c|}{ Torpor } & \multirow[b]{2}{*}{ P-value } & \multirow[b]{2}{*}{$\begin{array}{c}\text { Fold } \\
\text { change }\end{array}$} \\
\hline & $\begin{array}{l}\text { Standardized } \\
\text { average } \\
\text { retention time } \\
\text { (min) }\end{array}$ & $\begin{array}{l}\text { Standardized } \\
\text { average area }\end{array}$ & $\begin{array}{l}\text { Standard } \\
\text { deviation }\end{array}$ & $\begin{array}{c}\text { Standardized } \\
\text { average } \\
\text { retention } \\
\text { time (min) }\end{array}$ & $\begin{array}{l}\text { Standardized } \\
\text { average area }\end{array}$ & $\begin{array}{l}\text { Standard } \\
\text { deviation }\end{array}$ & & \\
\hline 690 & 11.1 & $2.26 \mathrm{E}-01$ & $9.62 \mathrm{E}-03$ & 11.2 & $3.43 E-01$ & $1.01 \mathrm{E}-01$ & 0.1138 & 1.52 \\
\hline 714 & 10.9 & 1.64E-01 & $2.84 \mathrm{E}-02$ & 10.8 & $7.56 \mathrm{E}-01$ & 1.84E-01 & 0.0053 & 4.61 \\
\hline 716 & 11.4 & $4.05 E+00$ & 4.57E-01 & 11.5 & $6.67 \mathrm{E}+00$ & $2.02 \mathrm{E}+00$ & 0.0942 & 1.64 \\
\hline 718 & 12.0 & $1.82 \mathrm{E}+00$ & $1.06 \mathrm{E}-01$ & 12.0 & $1.76 \mathrm{E}+00$ & $5.85 \mathrm{E}-01$ & 0.8852 & 0.97 \\
\hline
\end{tabular}




\begin{tabular}{|l|l|l|l|l|l|l|l|l|}
\hline 724 & 12.3 & $4.38 \mathrm{E}-02$ & $6.44 \mathrm{E}-03$ & 12.4 & $4.15 \mathrm{E}-02$ & $1.54 \mathrm{E}-02$ & 0.8179 & 0.95 \\
\hline 730 & 12.0 & $1.44 \mathrm{E}-01$ & $6.25 \mathrm{E}-03$ & 12.1 & $4.48 \mathrm{E}-01$ & $8.50 \mathrm{E}-02$ & 0.0035 & 3.12 \\
\hline 736 & 10.6 & $3.56 \mathrm{E}-02$ & $3.06 \mathrm{E}-03$ & 10.6 & $6.17 \mathrm{E}-02$ & $9.69 \mathrm{E}-03$ & 0.0114 & 1.73 \\
\hline 738 & 11.1 & $4.51 \mathrm{E}-01$ & $6.61 \mathrm{E}-02$ & 11.2 & $1.70 \mathrm{E}+00$ & $5.48 \mathrm{E}-01$ & 0.0173 & 3.77 \\
\hline 740 & 11.7 & $3.81 \mathrm{E}+00$ & $2.67 \mathrm{E}-01$ & 11.5 & $4.78 \mathrm{E}+00$ & $1.27 \mathrm{E}+00$ & 0.2659 & 1.25 \\
\hline 742 & 11.8 & $3.62 \mathrm{E}+00$ & $2.83 \mathrm{E}-01$ & 11.8 & $1.48 \mathrm{E}+01$ & $3.64 \mathrm{E}+00$ & 0.0060 & 4.09 \\
\hline 744 & 12.5 & $1.05 \mathrm{E}+01$ & $5.38 \mathrm{E}-01$ & 12.7 & $1.83 \mathrm{E}+01$ & $5.63 \mathrm{E}+00$ & 0.0759 & 1.74 \\
\hline 750 & 12.4 & $1.23 \mathrm{E}-01$ & $5.27 \mathrm{E}-03$ & 12.7 & $1.25 \mathrm{E}-01$ & $3.55 \mathrm{E}-02$ & 0.9184 & 1.02 \\
\hline 752 & 12.8 & $1.20 \mathrm{E}-01$ & $1.80 \mathrm{E}-02$ & 11.8 & $1.99 \mathrm{E}-01$ & $5.52 \mathrm{E}-02$ & 0.0771 & 1.66 \\
\hline 754 & 12.2 & $2.15 \mathrm{E}-01$ & $3.62 \mathrm{E}-03$ & 12.3 & $2.85 \mathrm{E}-01$ & $1.29 \mathrm{E}-01$ & 0.4027 & 1.32 \\
\hline 756 & 12.2 & $5.78 \mathrm{E}-02$ & $1.09 \mathrm{E}-02$ & 11.7 & $1.79 \mathrm{E}-01$ & $3.25 \mathrm{E}-02$ & 0.0036 & 3.10 \\
\hline 758 & 13.0 & $6.85 \mathrm{E}-02$ & $1.13 \mathrm{E}-02$ & 12.1 & $1.28 \mathrm{E}-01$ & $7.94 \mathrm{E}-03$ & 0.0017 & 1.87 \\
\hline 762 & 11.0 & $2.14 \mathrm{E}-01$ & $3.11 \mathrm{E}-02$ & 10.9 & $3.22 \mathrm{E}-01$ & $7.95 \mathrm{E}-02$ & 0.0937 & 1.50 \\
\hline 764 & 11.7 & $1.26 \mathrm{E}+01$ & $1.19 \mathrm{E}+00$ & 11.7 & $6.47 \mathrm{E}+00$ & $1.63 \mathrm{E}+00$ & 0.0064 & 0.51 \\
\hline 766 & 12.0 & $5.02 \mathrm{E}+00$ & $4.76 \mathrm{E}-01$ & 12.2 & $8.13 \mathrm{E}+00$ & $3.41 \mathrm{E}+00$ & 0.1926 & 1.62 \\
\hline 768 & 12.8 & $1.74 \mathrm{E}+01$ & $1.14 \mathrm{E}+00$ & 12.9 & $1.57 \mathrm{E}+01$ & $4.93 \mathrm{E}+00$ & 0.6077 & 0.91 \\
\hline 778 & 12.2 & $2.64 \mathrm{E}-01$ & $1.15 \mathrm{E}-02$ & 12.3 & $3.28 \mathrm{E}-01$ & $8.79 \mathrm{E}-02$ & 0.2786 & 1.24 \\
\hline 780 & 12.5 & $9.04 \mathrm{E}-02$ & $7.60 \mathrm{E}-03$ & 12.5 & $1.79 \mathrm{E}-01$ & $6.06 \mathrm{E}-02$ & 0.0657 & 1.98 \\
\hline 782 & 13.2 & $1.25 \mathrm{E}-01$ & $1.22 \mathrm{E}-02$ & 13.0 & $2.07 \mathrm{E}-01$ & $5.80 \mathrm{E}-02$ & 0.0731 & 1.66 \\
\hline 788 & 11.3 & $1.44 \mathrm{E}-01$ & $2.36 \mathrm{E}-02$ & 11.4 & $5.40 \mathrm{E}-01$ & $1.43 \mathrm{E}-01$ & 0.0091 & 3.75 \\
\hline 790 & 11.9 & $3.43 \mathrm{E}+00$ & $2.98 \mathrm{E}-01$ & 12.0 & $3.28 \mathrm{E}+00$ & $1.45 \mathrm{E}+00$ & 0.8698 & 0.96 \\
\hline 792 & 12.7 & $1.17 \mathrm{E}+01$ & $7.84 \mathrm{E}-01$ & 12.8 & $7.41 \mathrm{E}+00$ & $2.77 \mathrm{E}+00$ & 0.0633 & 0.64 \\
\hline
\end{tabular}

Table S. 3:4. Non-statistically significant PS peaks after student t-test analysis and Benjamini-Hochberg correction.

\begin{tabular}{|c|c|c|c|c|c|c|c|c|}
\hline \multirow[b]{2}{*}{$\mathrm{m} / \mathrm{z}$} & \multicolumn{3}{|c|}{ Control } & \multicolumn{3}{|c|}{ Torpor } & \multirow[b]{2}{*}{$\begin{array}{c}\text { P- } \\
\text { value }\end{array}$} & \multirow[b]{2}{*}{$\begin{array}{c}\text { Fold } \\
\text { change }\end{array}$} \\
\hline & $\begin{array}{c}\text { Standardized } \\
\text { average } \\
\text { retention } \\
\text { time (min) }\end{array}$ & $\begin{array}{l}\text { Standardized } \\
\text { average area }\end{array}$ & $\begin{array}{l}\text { Standard } \\
\text { deviation }\end{array}$ & $\begin{array}{l}\text { Standardized } \\
\text { average } \\
\text { retention } \\
\text { time (min) } \\
\end{array}$ & $\begin{array}{l}\text { Standardized } \\
\text { average area }\end{array}$ & $\begin{array}{l}\text { Standard } \\
\text { deviation }\end{array}$ & & \\
\hline 788 & 11.8 & 2.33E-01 & $3.13 \mathrm{E}-02$ & 11.7 & $6.17 \mathrm{E}-01$ & $1.97 \mathrm{E}-01$ & 0.0291 & 2.65 \\
\hline 790 & 12.3 & $1.64 \mathrm{E}-01$ & $4.85 E-03$ & 12.1 & $2.56 \mathrm{E}-01$ & $1.05 \mathrm{E}-01$ & 0.2062 & 1.55 \\
\hline 808 & 10.9 & $3.02 \mathrm{E}-01$ & 3.47E-02 & 10.9 & 4.67E-01 & $6.95 \mathrm{E}-02$ & 0.0213 & 1.55 \\
\hline 810 & 11.3 & $9.38 \mathrm{E}-02$ & 8.98E-03 & 11.3 & $2.51 \mathrm{E}-01$ & 4.27E-02 & 0.0033 & 2.68 \\
\hline 812 & 12.1 & $6.30 \mathrm{E}-01$ & 8.31E-02 & 11.9 & 4.77E-01 & $1.64 \mathrm{E}-01$ & 0.2221 & 0.76 \\
\hline 836 & 12.1 & $1.43 E+00$ & 1.64E-01 & 11.9 & $1.05 E+00$ & $2.89 \mathrm{E}-01$ & 0.1156 & 0.73 \\
\hline
\end{tabular}




\subsection{References}

(1) Muro, E.; Atilla-Gokcumen, G. E.; Eggert, U. S. Mol. Biol. Cell 2014, 25 (12), 1819-1823.

(2) Bou Khalil, M.; Hou, W.; Zhou, H.; Elisma, F.; Swayne, L. A.; Blanchard, A. P.; Yao, Z.; Bennett, S. A. L.; Figeys, D. Mass Spectrom. Rev. 2010, 29 (6), 877929.

(3) Ho, Y.-P.; Huang, P.-C. Rapid Commun. Mass Spectrom. 2002, 16 (16), 1582 1589.

(4) Li, X.; Garrity, A. G.; Xu, H. J. Physiol. 2013, 591 (18), 4389-4401.

(5) Deep, G.; Schlaepfer, I. R. Int. J. Mol. Sci. 2016, 17 (7).

(6) Fhaner, C. J.; Liu, S.; Ji, H.; Simpson, R. J.; Reid, G. E. Anal. Chem. 2012, 84 (21), 8917-8926.

(7) Murphy, R. C.; Harrison, K. A. Mass Spectrom. Rev. 1994, 13 (1), 57-75.

(8) Hsu, F. F.; Bohrer, A.; Turk, J. Biochim. Biophys. Acta 1998, 1392 (2-3), 202216.

(9) Slotte, J. P. Prog. Lipid Res. 2013, 52 (4), 424-437.

(10) Han, X.; Holtzman, D. M.; McKeel, D. W. J. Neurochem. 2001, 77 (4), 1168-1180.

(11) Han, X.; M Holtzman, D.; McKeel, D. W.; Kelley, J.; Morris, J. C. J. Neurochem. 2002, 82 (4), 809-818.

(12) Kennedy, M. A.; Moffat, T. C.; Gable, K.; Ganesan, S.; Niewola-Staszkowska, K.; Johnston, A.; Nislow, C.; Giaever, G.; Harris, L. J.; Loewith, R.; Zaremberg, V.; Harper, M.-E.; Dunn, T.; Bennett, S. A. L.; Baetz, K. Sci. Rep. 2016, 6, 19332.

(13) Storey, K. B. J. Exp. Biol. 2015, 218 (1), 150-159.

(14) Horman, S.; Hussain, N.; Dilworth, S. M.; Storey, K. B.; Rider, M. H. Comp. Biochem. Physiol. Part B Biochem. Mol. Biol. 2005, 142 (4), 374-382.

(15) Morin, P.; Storey, K. B. Biochim. Biophys. Acta - Gene Struct. Expr. 2005, 1729 (1), 32-40.

(16) Morin, P.; Storey, K. B. Int. J. Dev. Biol. 2009, 53 (2-3), 433-442.

(17) Pan, Q.; Li, M.; Shi, Y.-L.; Liu, H.; Speakman, J. R.; Wang, D.-H. Lipids 2014, 49 (7), 715-730.

(18) Johnson, R. J.; Stenvinkel, P.; Martin, S. L.; Jani, A.; Sánchez-Lozada, L. G.; Hill, J. O.; Lanaspa, M. A. Obesity (Silver Spring). 2013, 21 (4), 659-664.

(19) Wijenayake, S.; Tessier, S. N.; Storey, K. B. J. Therm. Biol. 2017, 69, 199-205.

(20) McMullen, D. C.; Hallenbeck, J. M. J. Comp. Physiol. B 2010, 180 (6), 927-934. 
(21) Bligh, E. G.; Dyer, W. J. Can. J. Biochem. Physiol. 1959, 37 (8), 911-917.

(22) Canez, C. R.; Shields, S. W. J.; Bugno, M.; Wasslen, K. V.; Weinert, H. P.; Willmore, W. G.; Manthorpe, J. M.; Smith, J. C. Anal. Chem. 2016, 88 (14), 69967004.

(23) Armbruster, D. A.; Pry, T. Clin. Biochem. Rev. 2008, 29 Suppl 1, S49-52.

(24) Xu, P.; Baldridge, R. D.; Chi, R. J.; Burd, C. G.; Graham, T. R. J. Cell Biol. 2013, 202 (6), 875-886.

(25) Watson, H. Essays Biochem. 2015, 59, 43-69.

(26) Aloia, R. C. Comp. Biochem. Physiol. 1981, 68B, 203-208.

(27) Dawaliby, R.; Trubbia, C.; Delporte, C.; Noyon, C.; Ruysschaert, J.-M.; Van Antwerpen, P.; Govaerts, C. J. Biol. Chem. 2016, 291 (7), 3658-3667. 


\section{Chapter 4. Analysis of glycerophospholipid dynamics in Rana sylvatica leg muscle and liver tissue during extreme temperature fluctuations via HPLC-MS/MS}

\subsection{Abstract}

Freeze tolerance is a specific adaptation within

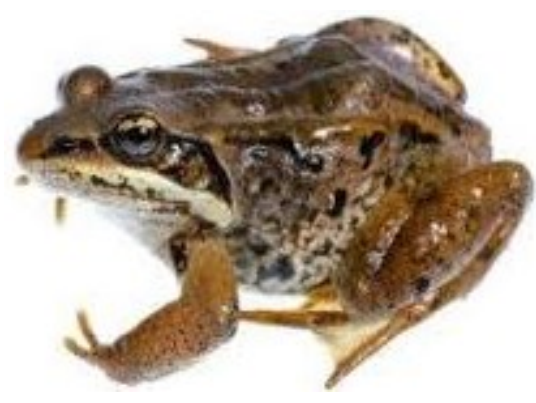
certain animals that gives them the ability to cope with cold climates by allowing some of their tissues to tolerate freezing. Liver and leg muscle tissues from Rana sylvatica (wood frog) were chosen as study models for freeze tolerance to observe and contrast any cold coping differences in vital versus non-vital organs. We chose to target three different subclasses of glycerophospholipids as well as sphingomyelin to observe how the major constituents of cell membranes change when the animal adapts to a colder climate. Lipids were extracted using a modified Bligh and Dyer extraction and high-performance liquid chromatography in conjunction with tandem mass spectrometry (HPLC-MS/MS) was used in the separation, detection, and analyses of the lipid species. It was observed that the wood frog increases the fluidity of its liver membranes during freezing by increasing the concentration of more highly unsaturated lipids. This increased fluidity would presumably preserve enzymatic processes at the cell surface and permit continued metabolism in the tissue at low temperatures. In contrast, the results from wood frog leg muscle study did not show appreciable glycerophospholipid dynamics at low temperature, demonstrating a difference in lipidomic dynamics in non-critical tissues. 


\subsection{Introduction}

During the winter months, many species are challenged with shortages of food, reduced photoperiods, and cold temperatures. Some animals migrate to escape cold temperatures while others have adapted to unusual energy-saving strategies. Certain mammals enter a period of hibernation during the winter to cope with reduced access to food and cold. Ectothermic species, which control body temperature mostly by external sources of heat, go through quite different cold coping mechanisms to survive winter: freeze tolerance and freeze avoidance. These two strategies are generally mutually exclusive; however, there are a few species that can utilize either modality depending upon existing physiological-environmental conditions ${ }^{1}$. Both freeze avoidance and freeze tolerance are effective cold-coping strategies; however, it is not entirely understood why a particular species adopts one over the other. It has been observed that freeze tolerance is particularly advantageous in habitats where winter temperatures are relatively mild and unpredictable ${ }^{2}$.

The formation of an ice nucleus is necessary for water to begin to freeze. When pure water is cooled to $0^{\circ} \mathrm{C}$, it does not spontaneously freeze. Instead water molecules form clusters where they are arranged like ice crystals but remain in a liquid state; this happens due to density fluctuations in liquid water ${ }^{3}$. This process is called supercooling. A random motion of water molecules triggers localised fluctuations in the density and orientation of the water molecules changes generating energy for ice nuclei formation. Impurities or foreign particles in the water help initiate the crystallization process ${ }^{4}$. If the cluster exists long enough for other water molecules to bind before the same random fluctuations cause it to break apart, water freezes ${ }^{5}$. Freeze avoidance is a survival 
strategy that involves preventing body fluids from freezing by limiting significant ice crystal nucleation using antifreeze proteins and glycoproteins. Antifreeze proteins and glycoproteins, found in polar marine fishes ${ }^{6}$ and insects ${ }^{7,8}$, coat growing ice crystals and prevent them from growing to a size where they would not be able to tolerate body cells freezing.

Freeze tolerance is defined as the ability of ectothermic vertebrates (e.g., frogs ${ }^{9,10}$ ), invertebrates (e.g., insects ${ }^{11}$ ), and marine invertebrates (e.g., mussels ${ }^{12}$ ) to survive at temperatures below zero degrees Celsius by using the specific physiological mechanisms that prevent the formation of intracellular ice ${ }^{13}$. The freezing point inside the cells is lowered by concentrating osmolytes like glycerol and glucose in the cells whereas ice formation outside the cells is encouraged by ice nucleators. Ice formation outside the cells also causes the remaining solutes to concentrate in the extracellular fluids, which creates an osmotic pressure gradient, this causes more water to move out of the cells, further concentrating the intracellular fluid and reducing its freezing point to a greater extent ${ }^{14}$.

The wood frog (Rana sylvatica) is common to North America and is frequently used as a model organism to study freeze tolerance. Like many other northern frogs, they enter a dormant period during the winter months underneath the soil close to its surface; wood frogs are able to tolerate the freezing of their blood and other tissues ${ }^{15,16}$. To prepare itself for cold temperatures, the wood frog accumulates urea in its tissues. Additionally, in response to the internal ice formation, liver glycogen is converted to glucose in large amounts. Urea and glucose act as cryoprotectants to limit the amount of ice that forms and to reduce osmotic shrinkage of cells ${ }^{17,18}$. Wood frogs can survive many freeze-thaw 
cycles throughout winter months, so long as no more than $65 \%$ of their total body water freezes ${ }^{10}$.

Glycerophospholipids are glycerol-based phospholipids that form one of the largest and most important classes of natural lipids. They are essential components of cell membranes and are found in small concentrations in other parts of cells. The general structure of a glycerophospholipid is shown in Figure 4:1(a). Some common glycerophospholipid head groups include phosphatidylethanolamine ( $\left.\mathrm{PE},-\mathrm{CH}_{2} \mathrm{CH}_{2} \mathrm{~N}^{+} \mathrm{H}_{3}\right)$, phosphatidylcholine (PC, $\left.-\mathrm{CH}_{2} \mathrm{CH}_{2} \mathrm{~N}^{+}\left(\mathrm{CH}_{3}\right)_{3}\right)$ and phosphatidylserine (PS, $\left.\mathrm{CH}_{2} \mathrm{CH}\left(\mathrm{N}^{+} \mathrm{H}_{3}\right) \mathrm{COOH}\right)$. Two aliphatic groups are also linked to the glycerol backbone either as a fatty acyl forming an ester bond or as a fatty alcohol forming an ether bond. When a vinyl ether bond is formed with the glycerol backbone, the lipid is referred to as a plasmalogen, and when an aliphatic group is missing from the glycerol backbone, the lipid is denoted as a lyso species. Sphingomyelin (SM), another type of lipid found in animal cell membranes and shown in Figure 4:1(b), is a sphingolipid consisting of a phosphocholine head group, a sphingosine, and a fatty acid. Due to the fact that they contain phosphocholine, they are classified along with glycerophospholipids as phospholipids for the purposes of this study.

The main function of glycerophospholipids is to serve as a structural component of biological membranes. Due to their amphipathic nature, they arrange themselves into bilayer structures where the hydrophobic tails point to each other and form a fatty, hydrophobic center. The polar head groups are located at the inner and outer surfaces of the bilayer forming a membrane. The molecular composition of a given bilayer varies with cell and tissue type in an organism dependent manner ${ }^{19}$. Furthermore, lipid compositions 
change temporally in response to changes in the environment ${ }^{20,21}$. In mammalian cells, the outer layer of the plasma membrane contains PC and sphingomyelin predominantly, whereas PS and PE are found on the inner layer of the bilayer ${ }^{22}$.

The fluidity of biological membranes is affected by the aliphatic chain length and degree of unsaturation of the constituent lipids as well as temperature. Membranes are more fluid as the aliphatic chain length decreases or when the temperature or the degree of unsaturation increases ${ }^{23}$. To be able to adjust to cold temperature conditions and maintain optimal membrane fluidity, cells regulate the expression of lipid desaturases ${ }^{24}$. This provides an essential balance between saturated and unsaturated membrane lipids $^{25}$.

The identity of the head group also influences fluidity. PE and PC lipids have different shapes; this also affects the fluidity of membranes; PE is conical while PC is cylindrical. The conical shape of PE prevents the close packing of lipids, allowing for more molecular movement within the membrane and, therefore, the increased fluidity of the membranes have come from research on bacteria ${ }^{26}$. However, despite these studies in the literature, the biomolecular mechanisms that regulate changes in membrane fluidity have not been fully characterized ${ }^{25}$.

Here we report an investigation into the glycerophospholipid dynamics that occur in wood frog tissues as the temperature is varied between normal and freezing conditions. We also contrast glycerophospholipid concentration changes in a vital organ (the liver) versus a non-vital organ (leg muscle tissue) during hypothermic stress. PC, PE, PS and SM lipid classes were investigated quantitatively with mass spectrometry via specific precursor ion and neutral loss scanning modes. 


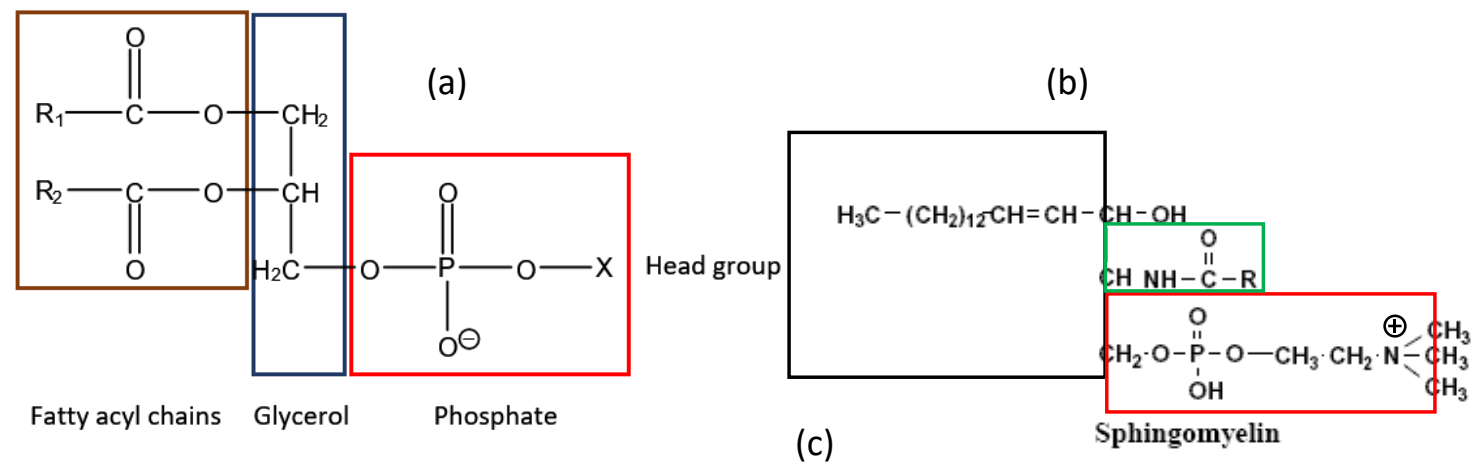

$\mathrm{PC} / \mathrm{SM}$

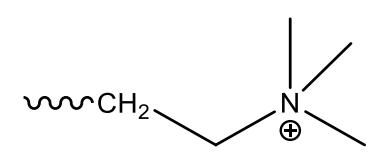

PE

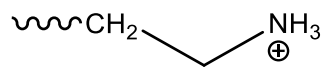

PS<smiles>CCCC(CC)C(=O)O</smiles>

Figure 4:1. (a) The generic structure of a glycerophospholipid; $R 1$ and $R 2$ are two nonpolar aliphatic chains (brown box), the core of the molecule is glycerol (blue box), a phosphate bound to a polar head group X (red box). Glycerol group carbons from the top are sn-1, sn-2, and sn-3 (b) Structure of sphingomyelin; 18-carbon amino alcohol (sphingosine) (black box) binds to a phosphocholine head group (red box) and a fatty acid group (green box). (c) head groups that are investigated in this work.

\subsection{Experimental Procedures}

Sample preparation: Control and dehydrated Rana sylvatica leg muscle and liver tissues were obtained from collaborative work with Dr. Kenneth Storey at Carleton University. Male wood frogs were collected from breeding ponds in the Ottawa area in the spring of 2007. Matthan et al., 2010 have demonstrated that storage at $-80^{\circ} \mathrm{C}$ up to 10 years does not significantly influence serum cholesteryl ester, triglyceride, and phospholipid fatty acid profiles $^{27}$. All frogs were washed in a tetracycline bath and held at $5^{\circ} \mathrm{C}$ for $1-2$ weeks; several frogs were sacrificed at this point as a control group. Freezing was performed by 
placing frogs in plastic containers on a layer of damp paper towel and incubating them at $-4.0^{\circ} \mathrm{C}$. The frogs were cooled for 45 minutes to allow the body temperatures to chill to sub-zero values and ice nucleation to occur. The temperature was then raised to $-2.5^{\circ} \mathrm{C}$ for a 24 hour period to obtain dehydrated tissue samples ${ }^{28}$.

All tissues were weighted to approximately $0.1 \mathrm{~g}$ and crushed with a mortar and pestle in the presence of liquid nitrogen. Then samples were placed into Eppendorf tubes, and $1 \mathrm{~mL}$ of $2 \%$ acetic acid in methanol $(\mathrm{AcMeOH})$ was added. The liver samples were sonicated on ice using a 130-watt ultrasonic processor sonicator (model: VCX130, from Thomas Scientific, Swedesboro, NJ, USA) Samples were sonicated for 9 minutes with the pulse on for 20 s and pulse off for 10 s with $50 \%$ amplitude. The leg muscle samples were first homogenized with a tissue homogenizer for 2 minutes on medium speed while on ice. The samples were then sonicated on ice as described above for 3 minutes.

Lipid extraction: Lipids were extracted via a modified Bligh and Dyer method ${ }^{29}$. Each sample was transferred to a $10 \mathrm{~mL}$ glass Kimble tube ("extraction tube") containing 3.2 $\mathrm{mL}$ of filtered $0.1 \mathrm{M} \mathrm{Na}$ acetate. The Eppendorf tube that contained the sample was rinsed 3 times with $1 \mathrm{~mL}$ of $2 \% \mathrm{AcMeOH}$, and each rinsing was transferred to extraction tube (the total volume of $2 \% \mathrm{AcMeOH}$ was $4 \mathrm{~mL}$ ). $60 \mu \mathrm{L}$ of a $1 \mathrm{mM}$ internal standard (C13:0 lysophosphatidylcholine) was added to the extraction tube followed by $3.8 \mathrm{~mL}$ of chloroform. The extraction tube was then vortexed, swirled and inverted 3 times, followed by a centrifugation step for 2 minutes at $2000 \mathrm{rpm}$ and $4^{\circ} \mathrm{C}$. The bottom chloroform phase was collected with a Pasteur pipette and transferred to a new $10 \mathrm{~mL}$ glass Kimble tube ("collection tube"). The extraction was repeated 3 more times with $2 \mathrm{~mL}$ of chloroform that was added to the collection tube. The chloroform was then evaporated under a stream of 
nitrogen gas until a dark yellow oily residue remained. Frog leg muscle lipid samples were resuspended in $300 \mu \mathrm{L}$ and frog liver lipid samples were resuspended in $1200 \mu \mathrm{L} 10 \mathrm{mM}$ ammonium acetate in absolute $\mathrm{EtOH}$. The samples were incubated at $30^{\circ} \mathrm{C}$ for 10 minutes to dissolve the lipids and centrifuged for 1 minute at $2000 \mathrm{rpm}$ and $4^{\circ} \mathrm{C}$. Samples transferred to amber glass tubes ("storage tubes"). The storage tubes were flushed with $\mathrm{N}_{2}$ gas and stored at $-20^{\circ} \mathrm{C}$ if analyzed within a week or at $-80^{\circ} \mathrm{C}$ if stored for longer periods of time. When ready for analysis, a sample of interest was taken out of the freezer and a small aliquot was further diluted in $10 \mathrm{mM}$ ammonium acetate in absolute $\mathrm{EtOH}$ $(1: 40)$.

HPLC-ESI-MS/MS analysis: Reversed phase chromatography columns were constructed in-house. Briefly, $88 \mu \mathrm{L}$ of Kasil \#1 was mixed with $16 \mu \mathrm{L}$ of formamide in an Eppendorf tube and vortexed for a couple of minutes. A $15 \mathrm{~cm}$ long piece of fused silica tube with an inner diameter of $200 \mu \mathrm{m}$ was dipped to this Kasil/formamide mixture; silica tubes were left overnight to dry allowing frit to polymerize (coated tip facing downwards). These fritted columns were packed with $5.5 \mathrm{~cm}$ of $\mathrm{C} 4$ silica using a nitrogen pressure vessel and then fritted at the other end as well before use.

The sample mixture for separation and analysis on the HPLC-ESI-MS was prepared by using $12.5 \mu \mathrm{L}$ of the diluted extracted lipid sample and $37.5 \mu \mathrm{L}$ of $10 \mathrm{mM}$ ammonium acetate in MilliQ water. The sample was injected onto the column using a HPLC (Dionex Ultimate 3000) auto-sampler filled with the following HPLC solvents: solvent A (10 mM ammonium acetate in 30\% methanol/70\% MilliQ water), solvent B (10 $\mathrm{mM}$ ammonium acetate in $75 \%$ IPA $25 \%$ methanol), solvent $\mathrm{C}$ (unused), and solvent $\mathrm{D}$ (methyl tert-butyl ether (MTBE)). The gradient began at $100 \%$ solvent $A$ and gradually 
increased to $100 \%$ solvent B in 41.4 minutes; the run ended with $100 \%$ MTBE by 48 minutes until the end (the total run time was 60 minutes). The sample was introduced into the hybrid triple quadrupole linear ion trap mass spectrometer (AB Sciex QTRAP 4000, Framingham, MA) using an electrospray ionization source with an electrode with an inner diameter of $100 \mu \mathrm{m}$.

In order to differentiate between PC and SM lipids, the data was collected at low resolution to identify PC species and unit resolution to identify SM species. The lowresolution scan was from $\mathrm{m} / \mathrm{z} 400$ to 1000 and unit resolution scan was from $\mathrm{m} / \mathrm{z} 600$ to 900. Low-resolution neutral loss scans for $141 \mathrm{Da}$ and $185 \mathrm{Da}$ were used to identify PE and PS, respectively. The PE neutral loss scan was from m/z 300 to 900 while the PS neutral loss scan was from $\mathrm{m} / \mathrm{z} 400$ to 1100 . All scans were conducted in positive ion mode, and technical triplicate analyses were done for both control and hibernating samples.

Data analysis and statistical analysis: Analyst 1.5 .1 was used to extract a peak list data from chromatograms. The extracted peak lists were entered into MultiQaunt 2.1.1 software to obtain peak area values. Data standardization was achieved in two different ways: first, hibernating tissue mass and control tissue mass were standardized to each other, and all the peak areas were standardized to the area of the internal standard. Averages of all the peaks from technical replicates were calculated with their standard deviation values. To be able to identify all the significant changes between hibernating and control lipid concentration values; fold change was calculated as well as a student's t-test was performed $(\alpha=0.05)$. To control for false discovery rate, Benjamini-Hochberg 
(BH) method was performed with $\alpha=0.01^{30}$ and $p$-values from student's t-test and $\mathrm{BH}$ methods were compared.

\subsection{Results}

Different resuspension volumes were used for lipid extract because the leg muscle tissue has lower overall lipid concentrations compared to the liver tissue. Based on the signal intensity in MS, the concentrations of the lipids were adjusted with dilution. Based on lipids sample dilution data, frog liver has at least four times more lipids compared to frog leg muscle sample.

A total of 195 glycerophospholipids were identified in wood frog liver above the limit of quantification (LOQ) including 86 PC, 28 SM, 58 PE and 23 PS species. The wood frog leg muscle yielded 122 glycerophospholipids including 61 PC, 7 SM, 36 PE and 18 PS species. Both of these results are summarized in Figure 4:2. The overall phospholipid distribution of these 4 classes of lipids is similar between liver and leg tissues. Both tissues contain mostly PC, followed by PE, SM and then PS. The LOQ was calculated using established protocols for the instrument used in the experiments ${ }^{31}$.

In addition to identifying all of the quantifiable phospholipids, the data was used to monitor the temporal dynamics of these species in dehydrated versus control tissues. This was achieved by comparing the standardized area values and calculating $p$-values $(\alpha=0.05)$ using a t-test to identify any significant concentration changes. It was found that $26 \%$ of the phospholipids in the wood frog liver do not incur a significant concentration 
change following dehydration (Figure $4: 3$ bottom panel). The other $74 \%$ of the phospholipids were found to change in abundance in a statistically significant manner. Most of these lipids increased in concentration following dehydration while some appeared following dehydration. Only one species decreased after dehydration, and none of them disappeared, Figure 4:3.

To avoid any false discoveries and increase the confidence of our findings, the Benjamini-Hochberg $(\mathrm{BH})$ method was chosen with an $\alpha=0.01^{32}$. The $\mathrm{BH}$ procedure provides less stringent control of false positive (Type I) errors compared to the familywise error rate (FWER) controlling procedures (e.g., the Bonferroni correction), which controls the probability of at least one Type I error. The $\mathrm{BH}$ procedure has greater power at the cost of increased numbers of Type I errors ${ }^{33}$. If there is a large number of multiple comparisons and the significance level is expected to be relatively high, the Bonferroni correction may lead to a high rate of false negatives ${ }^{34}$.

The image on the bottom panel of Figure 4:3 shows significant phospholipid concentration changes in the dehydrated wood frog liver sample after applying the $\mathrm{BH}$ method correction. The significantly changing lipids have decreased quite substantially in comparison to traditional t-test value, from $74 \%$ to $26 \%$. It has been found that 26 phospholipids out of the 195 glycerophospholipids quantified in the wood frog liver encounter significant concentration changes during dehydration of the reptile at a $1 \%$ false discovery rate. 


\section{Phospholipids (195) in wood frog liver}

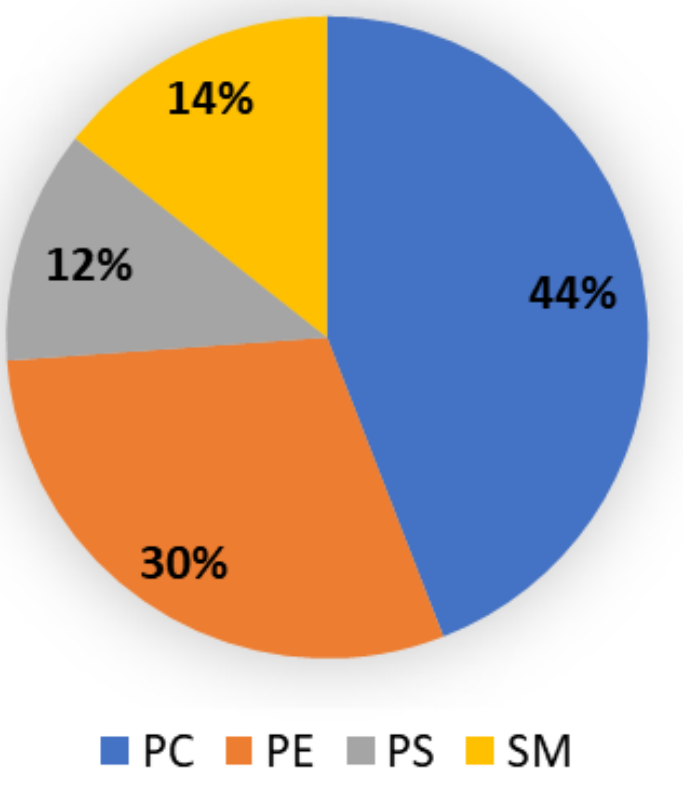

\section{Phospholipids (122) in wood frog leg}

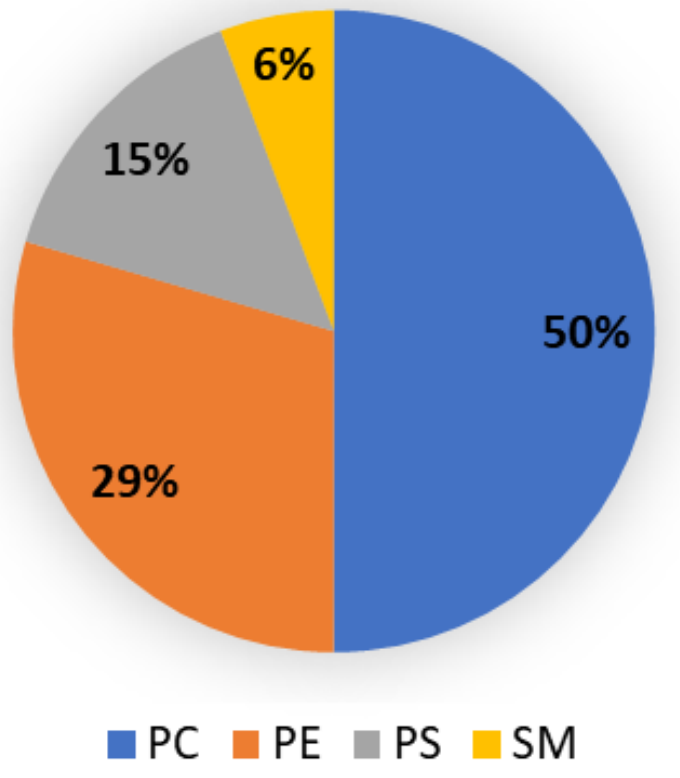

Figure 4:2. Relative distribution of glycerophospholipids found in wood frog liver and leg muscle samples. There are a total of 195 phospholipids found in wood frog liver samples above the limit of quantification (LOQ), and a total of 122 phospholipids found in wood frog leg samples above the LOQ. 


\section{Phospholipid conc. change in Dehydration}

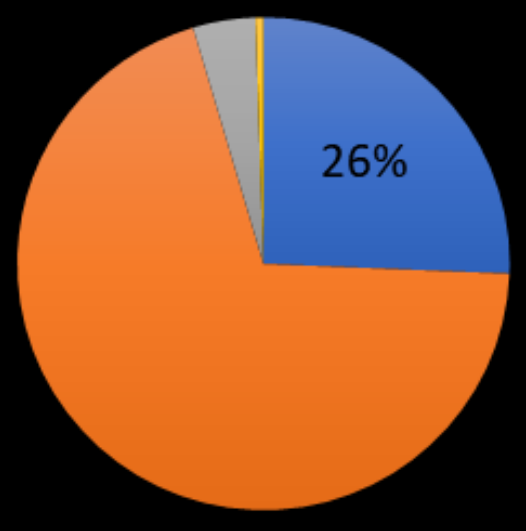

No significant change in Dehyd.

Increased in Dehyd.

- Appeared in Dehyd.

- Decreased in Dehyd.

Disapeared in Dehyd.

\section{After BH method}

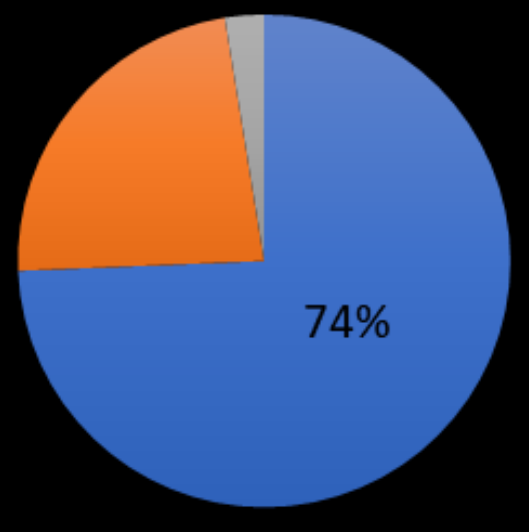

No significant change in Dehyd.

- Increased in Dehyd.

Appeared in Dehyd.

Decreased in Dehyd.

Disapeared in Dehyd.

Figure 4:3. Phospholipid concentrations are compared between control and dehydrated wood frog liver tissues. The bottom panel reveals the distribution after applying the false discovery rate correction using Benjamini-Hochberg (BH) method ( $\alpha=0.01$ ). 
The same type of experimental results for the wood frog leg muscle is summarized in Figure $4: 4$. It was found that $96 \%$ of the phospholipids in the wood frog leg muscle sample do not significantly change in concentration following dehydration. The other $4 \%$ of phospholipids revealed a significant concentration change via a t-test; where they both increased and decreased following dehydration. However, the significance of this result decreases from $4 \%$ to $0 \%$ after applying the $\mathrm{BH}$ correction. Since leg muscle is not a vital organ, it makes sense that membrane remodeling does not occur as the tissue adjust to colder temperatures.

24 out of the 86 quantifiable PC lipids found in the wood frog liver were discovered to significantly change in concentration as the organism adjusted to freezing temperatures, Figure 4:5. It is noteworthy that some of the PC species are increasing in abundance more than two-fold in response to the lower temperature.

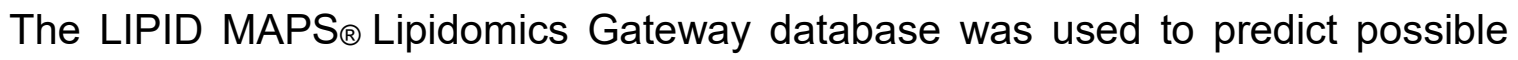
identities for the lipid masses that were measured in this work. Since the data produced in this research only yields nominal masses, a group of possible structures for each mass was identified. For example, considering only PC as a subclass, $\mathrm{m} / \mathrm{z} 818$ the possible structures that are reported in LIPID MAPS include PC(38:0), PC(39:7), PC(O-39:0), and $\mathrm{PC}(\mathrm{P}-40: 6)$; the length of the acyl chains, and the location of the double bonds is not known. There are also possible PE and PS species that could result in $\mathrm{m} / \mathrm{z} 818$. Tables 4:1 and 4:2 list possible structures of PC species quantified from wood frog liver samples. 


\section{Phospholipid conc. change in Dehydration}

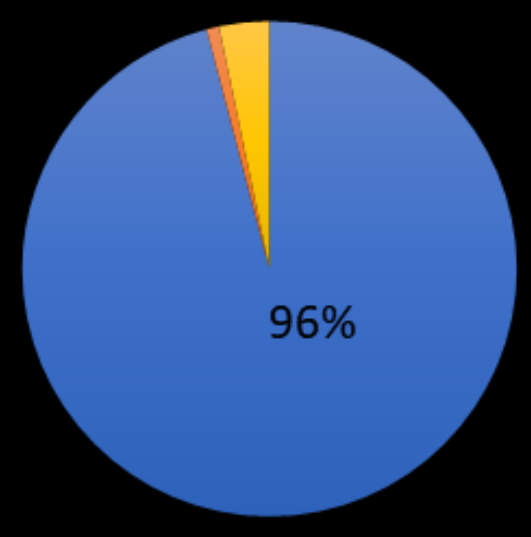

No significant change in Dehyd.

Increased in Dehyd.

- Appeared in Dehyd.

Disapeared in Dehyd.

Decreased in Dehyd.

\section{After BH method}

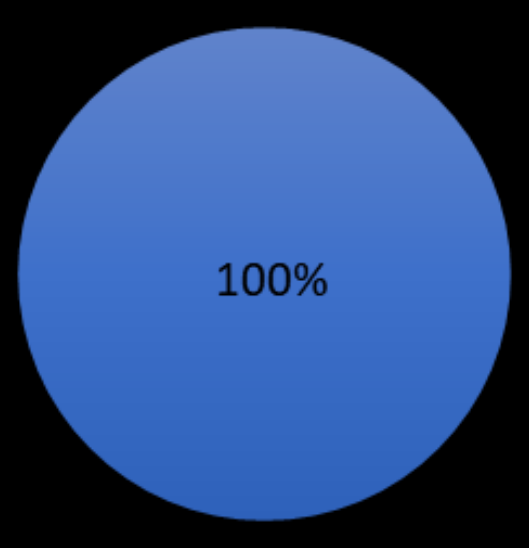

No significant change in Dehyd.

- Increased in Dehyd.

Appeared in Dehyd.

- Decreased in Dehyd.

Disapeared in Dehyd.

Figure 4:4. Phospholipid concentrations are compared between control and dehydrated wood frog leg muscle tissues. The bottom panel reveals the distribution after applying a false discovery rate correction using Benjamini-Hochberg (BH) method $(\alpha=0.01)$. 


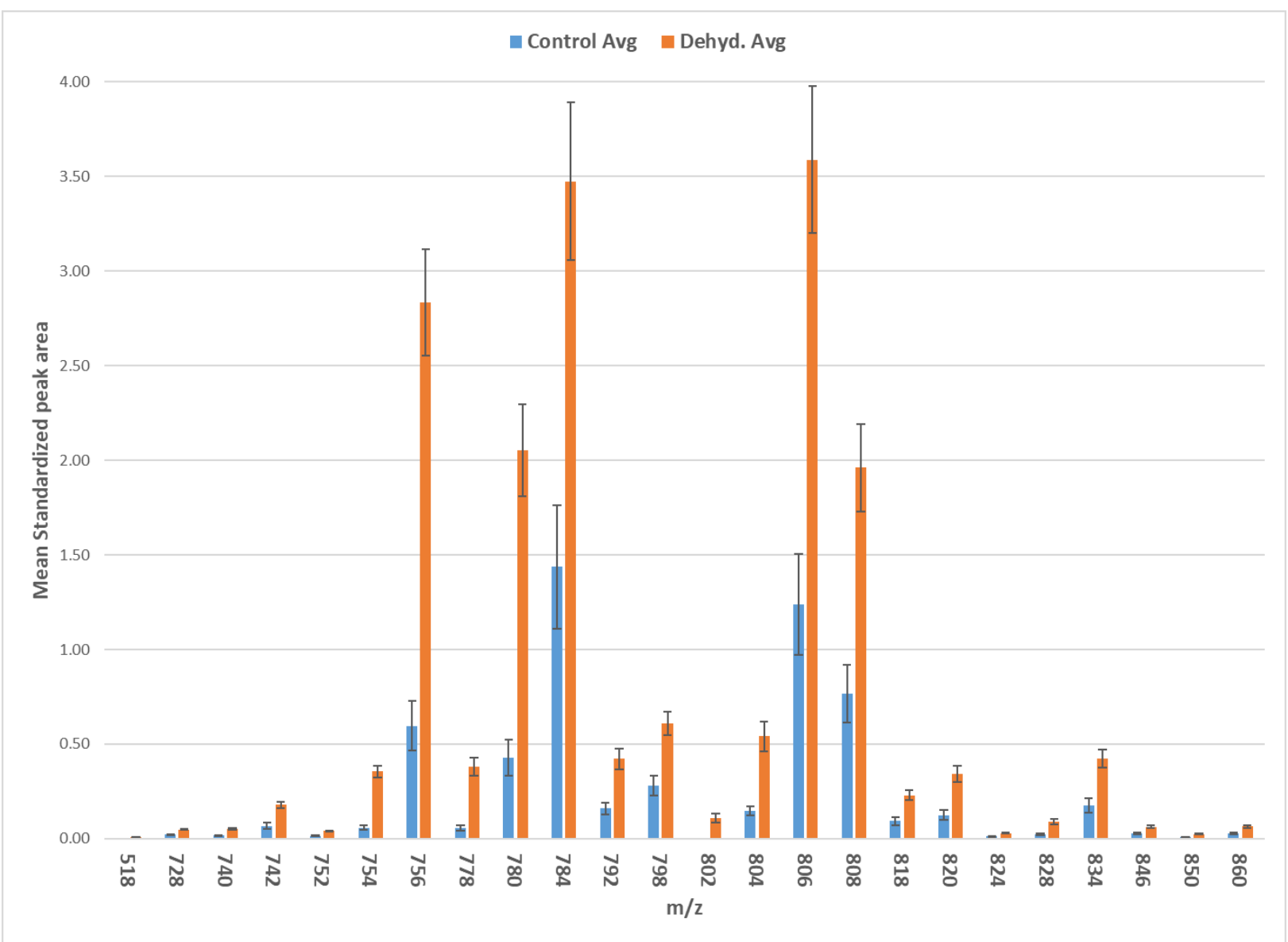

Figure 4:5. Relative abundances of 24 PC lipids in wood frog liver control versus dehydrated samples that change in a statistically significant manner after applying the $\mathrm{BH}$ correction.

Table 4:1 includes the 24 PC species that changed in concentration in a statistically significant manner following exposure to cold temperatures and Table 4:2 shows the remaining 24 PCs that demonstrate no significant correlation between abundance and temperature. It is noteworthy that there are more species with higher degrees of unsaturation in Table 4:1 compared to Table 4:2, which would suggest that the desaturation of membrane lipids is a mechanism of cold adaptation in the wood frog liver. 


\subsection{Discussion}

An increase in cellular membrane fluidity at colder temperatures is an established and important adaption mechanism in many different species ${ }^{24-26}$. In the literature, fluidity fluctuation is often attributed to differing ratios of PC, SM, and PE. For example, increasing relative abundances of $\mathrm{PC}$ over SM (or a combination of $\mathrm{PE}$ and $\mathrm{SM}$ ) or increasing ratios of $\mathrm{PE}$ alone relative to $\mathrm{PC}$ are indicative of higher fluidity ${ }^{35-38}$. Fajardo et al., 2011 developed a phospholipid fluidity index (PFI) and demonstrated that $\mathrm{PC} /(\mathrm{PE}+\mathrm{SM})$ is a better indication of membrane fluidity than ratios of $\mathrm{PC} / \mathrm{SM}$ and $\mathrm{PC} / \mathrm{PE}$ alone $^{36}$. Unfortunately, the $\mathrm{PC} /(\mathrm{PE}+\mathrm{SM})$ ratio has not gained too much popularity in membrane fluidity descriptions in the scientific literature, and the other two are used more often. Even though PC and SM have the same phosphate head group (choline), it has been suggested that PC is more hydrated than SM which would allow for SM to pack more tightly than $\mathrm{PC}^{39}$. The literature suggests that higher relative amounts of SM make membranes more rigid and less fluid $36,37,40$. However, many also have shown that there is no significant correlation between the PC/SM ratio and membrane fluidity ${ }^{35,38}$ and that SM plays a more critical role in conferring chemical resistance to oxidation and serving as a receptor molecule for extracellular ligands ${ }^{41}$. PC has a larger head group compared to $\mathrm{PE}\left(\mathrm{X}=-\mathrm{CH}_{2} \mathrm{CH}_{2} \mathrm{~N}^{+}\left(\mathrm{CH}_{3}\right)_{3}\right.$ in $\mathrm{PC}$ versus $\mathrm{X}=-\mathrm{CH}_{2} \mathrm{CH}_{2} \mathrm{~N}^{+} \mathrm{H}_{3}$ in $\left.\mathrm{PE}\right)$ and has a cylindrical shape; PE is a disordering lipid due to its conical shape which avoids close packing of lipids $^{35}$. Many agree that increasing amounts of PE correlates to increased membrane fluidity ${ }^{35,42-44}$, Dawaliby et al., 2016 states that PE is a key regulator of membrane fluidity 
Table 4:1. PC lipids that increase in response to freezing temperatures in the wood frog liver.

\begin{tabular}{|c|c|c|}
\hline $\begin{array}{l}\text { Phospholipid } \\
\text { class }\end{array}$ & $\begin{array}{l}\text { Low-resolution masses of } \\
\text { phospholipids }(\mathrm{m} / \mathrm{z})\end{array}$ & Possible structures of phospholipids \\
\hline PC & 518 & $\mathrm{PC}(18: 3)$ \\
\hline PC & 728 & $P C(32: 3), P C(P-33: 2)$ \\
\hline PC & 740 & $\mathrm{PC}(33: 4), \mathrm{PC}(0-34: 4), \mathrm{PC}(\mathrm{P}-34: 3)$ \\
\hline PC & 742 & $\mathrm{PC}(33: 3), \mathrm{PC}(0-34: 3), \mathrm{PC}(\mathrm{P}-34: 2)$ \\
\hline PC & 752 & $\mathrm{PC}(34: 5)$ \\
\hline PC & 754 & $\mathrm{PC}(34: 4), \mathrm{PC}(0-35: 4)$ \\
\hline PC & 756 & $P C(34: 3), P C(P-35: 2)$ \\
\hline PC & 778 & $\mathrm{PC}(36: 6)$ \\
\hline PC & 780 & $P C(36: 5)$ \\
\hline PC & 784 & $\mathrm{PC}(36: 3), \mathrm{PC}(\mathrm{P}-37: 2)$ \\
\hline PC & 792 & $\mathrm{PC}(37: 6), \mathrm{PC}(38: 6), \mathrm{PC}(\mathrm{P}-38: 5)$ \\
\hline PC & 798 & $\mathrm{PC}(37: 3), \mathrm{PC}(0-38: 3), \mathrm{PC}(\mathrm{P}-38: 2)$ \\
\hline PC & 802 & $\mathrm{PC}(37: 1), \mathrm{PC}(38: 8), \mathrm{PC}(0-38: 1), \mathrm{PC}(\mathrm{P}-38: 0)$ \\
\hline PC & 804 & $\mathrm{PC}(37: 0), \mathrm{PC}(38: 7), \mathrm{PC}(0-38: 0)$ \\
\hline PC & 806 & $\mathrm{PC}(38: 6)$ \\
\hline PC & 808 & $\mathrm{PC}(38: 5)$ \\
\hline PC & 818 & $\mathrm{PC}(38: 0), \mathrm{PC}(39: 7), \mathrm{PC}(0-39: 0), \mathrm{PC}(\mathrm{P}-40: 6)$ \\
\hline PC & 820 & $\mathrm{PC}(39: 6), \mathrm{PC}(0-40: 6), \mathrm{PC}(\mathrm{P}-40: 5)$ \\
\hline PC & 824 & $\mathrm{PC}(39: 4), \mathrm{PC}(0-40: 4), \mathrm{PC}(\mathrm{P}-40: 3)$ \\
\hline PC & 828 & $\mathrm{PC}(39: 2), \mathrm{PC}(40: 9), \mathrm{PC}(0-40: 2), \mathrm{PC}(\mathrm{P}-40: 1)$ \\
\hline PC & 834 & $\mathrm{PC}(40: 6)$ \\
\hline PC & 846 & $\mathrm{PC}(40: 0), \mathrm{PC}(41: 7), \mathrm{PC}(0-41: 0), \mathrm{PC}(\mathrm{P}-42: 6)$ \\
\hline PC & 850 & $\mathrm{PC}(41: 5), \mathrm{PC}(\mathrm{P}-42: 4)$ \\
\hline PC & 860 & $\mathrm{PC}(41: 0), \mathrm{PC}(42: 7), \mathrm{PC}(0-42: 0)$ \\
\hline
\end{tabular}


Table 4:2. PC lipids that do not increase in response to freezing temperatures in the wood frog liver.

\begin{tabular}{|c|c|c|}
\hline $\begin{array}{l}\text { Phospholipid } \\
\text { class }\end{array}$ & $\begin{array}{l}\text { Low-resolution masses of } \\
\text { phospholipids }(\mathrm{m} / \mathrm{z})\end{array}$ & Possible structures of phospholipids \\
\hline PC & 538 & $\mathrm{PC}(18: 0), \mathrm{PC}(0-19: 0), \mathrm{PC}(19: 0), \mathrm{PC}(0-20: 0)$ \\
\hline PC & 544 & $\operatorname{PC}(20: 4)$ \\
\hline PC & 660 & $\mathrm{PC}(27: 2)$ \\
\hline PC & 674 & $\mathrm{PC}(28: 2)$ \\
\hline PC & 702 & $\mathrm{PC}(30: 2), \mathrm{PC}(\mathrm{P}-31: 1)$ \\
\hline PC & 716 & $\mathrm{PC}(31: 2), \mathrm{PC}(0-32: 2), \mathrm{PC}(\mathrm{P}-32: 1)$ \\
\hline PC & 718 & $\mathrm{PC}(31: 1), \mathrm{PC}(0-32: 1), \mathrm{PC}(\mathrm{P}-32: 0)$ \\
\hline PC & 720 & $\mathrm{PC}(31: 0), \mathrm{PC}(0-32: 0)$ \\
\hline PC & 730 & $\mathrm{PC}(32: 2), \mathrm{PC}(0-33: 2), \mathrm{PC}(\mathrm{P}-33: 1)$ \\
\hline PC & 732 & $\mathrm{PC}(32: 1), \mathrm{PC}(0-33: 1), \mathrm{PC}(\mathrm{P}-33: 0)$ \\
\hline PC & 734 & $\mathrm{PC}(32: 0), \mathrm{PC}(0-33: 0)$ \\
\hline PC & 830 & $\mathrm{PC}(39: 1), \mathrm{PC}(40: 8), \mathrm{PC}(0-40: 1), \mathrm{PC}(\mathrm{P}-40: 0)$ \\
\hline PC & 840 & $\mathrm{PC}(40: 3)$ \\
\hline PC & 842 & $\mathrm{PC}(40: 2)$ \\
\hline PC & 844 & $\mathrm{PC}(40: 1)$ \\
\hline PC & 852 & $\mathrm{PC}(41: 4), \mathrm{PC}(42: 11), \mathrm{PC}(0-42: 4)$ \\
\hline PC & 854 & $\mathrm{PC}(41: 3), \mathrm{PC}(42: 10), \mathrm{PC}(0-42: 3), \mathrm{PC}(\mathrm{P}-42: 2)$ \\
\hline PC & 856 & $\mathrm{PC}(41: 2), \mathrm{PC}(42: 9), \mathrm{PC}(0-42: 2), \mathrm{PC}(\mathrm{P}-42: 1)$ \\
\hline PC & 864 & $\mathrm{PC}(42: 5)$ \\
\hline PC & 866 & $\mathrm{PC}(42: 4)$ \\
\hline PC & 876 & $\mathrm{PC}(43: 6)$ \\
\hline PC & 880 & $\mathrm{PC}(43: 4), \mathrm{PC}(0-44: 4)$ \\
\hline PC & 882 & $\mathrm{PC}(43: 3)$ \\
\hline PC & 900 & $\operatorname{PC}(44: 1)$ \\
\hline
\end{tabular}


in eukaryotic cells ${ }^{45}$. Lastly, it is not quite understood how PS affects the fluidity of biological membranes; in the study by Tsakiris and Deliconstantinos, 1984 they suggest that PS may increase the membrane fluidity ${ }^{46}$.

Table 4:3. Relative percentages and ratios of phospholipids in the liver and leg muscle membranes from wood frog tissues between control and dehydration conditions.

\begin{tabular}{ccccc}
\hline & Liver Control & Liver Dehyd. & Leg muscle & Leg muscle \\
& & & control & Dehyd. \\
\hline $\mathrm{PC}$ & $44.4 \%$ & $44.1 \%$ & $50.0 \%$ & $50.0 \%$ \\
\hline $\mathrm{PE}$ & $28.3 \%$ & $29.7 \%$ & $29.5 \%$ & $29.5 \%$ \\
\hline $\mathrm{PS}$ & $12.3 \%$ & $11.8 \%$ & $14.8 \%$ & $14.8 \%$ \\
\hline $\mathrm{SM}$ & $15.0 \%$ & $14.4 \%$ & $5.7 \%$ & $5.7 \%$ \\
\hline $\mathrm{PC} / \mathrm{SM}$ & 3.00 & 3.07 & 8.71 & 8.71 \\
\hline $\mathrm{PC} / \mathrm{PE}$ & 1.53 & 1.48 & 1.69 & 1.69 \\
\hline $\mathrm{PC} /(\mathrm{PE}+\mathrm{SM})$ & 1.01 & 1.00 & 1.42 & 1.42 \\
\hline
\end{tabular}

The membrane fluidity of the frog tissues in this study can be postulated from Table 4:3. It is observed that all of the ratio values for leg muscle are higher than for liver. PC/SM and $\mathrm{PC} /(\mathrm{PE}+\mathrm{SM})$ values seem to indicate that there is more fluidity in the leg muscle tissue membranes compared to liver membranes. This result is in contradiction with PC/PE results which indicate leg muscle rigidity compared to liver tissue. Perhaps, SM does not play a significant role in membrane fluidity as motioned above. Also, in adaptation to the cold temperatures, there is no difference in membrane fluidity in the leg muscle membrane according to the values in Table 4:3. For liver, the membrane fluidity ratios are smaller in dehydration compared to control in the case of PC/PE and 
$\mathrm{PC} /(\mathrm{PE}+\mathrm{SM})$ and larger in dehydration than control in the case of $\mathrm{PC} / \mathrm{SM}$; indicating a small increase in membrane fluidity in dehydration. These results are consistent with the results obtained by Reynolds et al., 2014 were they studied wood frog natural dehydration and laboratory dehydration as well as the difference in dehydration in Alaskan frog compared to Ohioan frog ${ }^{35}$. In this study and the study by Reynolds et al., 2014, the fluidity values of the laboratory environment dehydration do not change significantly, but a small decrease in PC/PE ratios is observed in both studies when compared to control samples. Also, there is no striking difference in individual phospholipid amounts in liver tissue in adaption to cold. However, PE is changing the most with an increase in the concentration of $1.4 \%$ while the rest of the phospholipids in the study decreased by less than $1 \%$. These results indicate that the frog has adapted to colder temperatures by increasing PE concentrations relative to other phospholipids in the membranes of its liver.

The activity of enzyme desaturase proteins is another well-characterized cold stress response studied mainly in bacterial species ${ }^{24,25}$. A fatty acid desaturase is an enzyme that removes two hydrogen atoms from a fatty acid, forming a carbon-carbon double bond. While the exact location of double bonds in the fatty acid chains is hard to determine due to isomeric nature of phospholipids, the total amount of double bonds in a certain phospholipid is easily calculated via its nominal mass. Table $4: 4$ summarizes the number of double bonds found in 24 significant and 24 non-significant PCs out of the 86 quantifiable PCs in wood frog liver tissue membranes (diacyl structure for all the fatty acid chains is assumed). It can be concluded from Table 4:4 that non-significant PCs are the species that do not change in a significant manner in the wood frog as it adapts to the cold, and that these PC have relatively low amounts of double bonds compared to 
significant PCs. On the other hand, PCs that change significantly have a higher number of double bonds compared to non-significant PCs. Our data suggest that glycerophospholipids become more unsaturated as an adaptation to freeze tolerance in liver tissue membranes. This result is consistent with previous results that demonstrated an increase in desaturase protein activity in response to cold stress observed in bacterial species. However, the mechanisms of desaturation may differ between bacterial and frog liver membranes.

Table 4:4. Number of double bonds found in 24 significant and 24 most non-significant PCs out of 88 total quantifiable PCs in wood frog liver tissue membranes. This table uses data from tables 4:1 and 4:2 and assumes diacyl structure for all the fatty acid chains.

\begin{tabular}{ccc}
\hline $\begin{array}{c}\text { Number of double bonds in } \\
\text { diacyl fatty acid chains }\end{array}$ & Number of significant PCs & Number of non-significant \\
\hline 6 & 5 & PCs \\
\hline 5 & 4 & 1 \\
\hline 4 & 3 & 4 \\
\hline 3 & 6 & 3 \\
\hline 2 & 1 & 7 \\
\hline 1 & 1 & 5 \\
\hline 0 & 4 & 3 \\
\hline
\end{tabular}

Other factors that can affect membrane fluidity are the incorporation of branchedchain fatty acids (BCFA), shortening the fatty acid chain length, changing the cis/trans fatty acid ratio, and changing the sterol/phospholipid ratio ${ }^{47}$. BCFA are usually saturated fatty acids with one or more methyl branches on the carbon chain, these kinds of fatty acids are most often found in bacteria ${ }^{48}$. Shortening the fatty acid chain length is only 
possible if the cells are actively growing ${ }^{49}$ which is not the case with a wood frog during freezing. As mentioned above, mass spectrometry studies do not readily reveal the locations of double bonds in the fatty acid chain, so identification of a double bond location as well as whether the bonds are cis or trans is beyond the scope of this work. The most common animal sterol cholesterol is frequently investigated when studying membrane fluidity ${ }^{35}$, however, this requires additional analysis using gas chromatography-MS and is beyond the scope of this work. For future studies, these analyses could be conducted to investigate the effects of saturation location and stereochemistry as well as sterol incorporation on membrane fluidity in response to cold stress.

\subsection{Conclusion}

This study elucidated novel freeze tolerance biological response employed by the wood frog in adaption to cold stress. In addition to dehydration via concentrating urea and glucose in its cells ${ }^{17,18}$, the wood frog also exhibits increased relative abundances of unsaturated phospholipids in its membranes to maintain the fluidity of the membrane and to avoid freezing of the cells. Significantly different levels of phospholipid unsaturation were observed in liver tissue, but not in leg muscle tissue. This is likely due to the liver being a vital organ; freezing of the liver would likely cease its function leading to the death of the organism. Conversely, the leg muscle is considered a non-vital organ and is part of the $65 \%$ of the body that freezes during freeze tolerance. With these adaptations, the wood frog may survive many freeze-thaw cycles during its lifetime without significant physiological deterioration. 


\subsection{Acknowledgments}

Frog tissues for this study were generously provided by Dr. Kenneth Storey, Carleton University. Financial support to JCS and KBS via NSERC Discovery Grants funded these discoveries and is gratefully acknowledged.

\subsection{References}

(1) Costanzo, J. P.; Lee, R. E. J. Exp. Biol. 2013, 216 (11), 1961-1967.

(2) Sinclair, B. J.; Addo-Bediako, A.; Chown, S. L. Biol. Rev. Camb. Philos. Soc. 2003, 78 (2), 181-195.

(3) Debenedetti, P. G. J. Phys. Condens. Matter 2003, 15, 1669-1726.

(4) Kawahara, H. In Advanced Topics on Crystal Growth; InTech, 2013; pp 119-143.

(5) Clarke, A. Principles of Thermal Ecology: Temperature, Energy, and Life; Oxford University Press, 2017; Vol. 1.

(6) Kristiansen, E.; Zachariassen, K. E. Cryobiology 2005, 51 (3), 262-280.

(7) Hakim, A.; Nguyen, J. B.; Basu, K.; Zhu, D. F.; Thakral, D.; Davies, P. L.; Isaacs, F. J.; Modis, Y.; Meng, W. J. Biol. Chem. 2013, 288 (17), 12295-12304.

(8) Duman, J. G. Annu. Rev. Physiol. 2001, 63 (1), 327-357.

(9) Hawkins, L. J.; Storey, K. B. J. Comp. Physiol. B 2018, 188 (1), 113-125.

(10) Storey, K. B.; Storey, J. M. Annu. Rev. Physiol. 1992, 54 (1), 619-637.

(11) Storey, K. B.; Storey, J. M. Can. J. Zool. 2012, 90 (4), 456-475.

(12) Storey, K. B.; Storey, J. M. Annu. Rev. Ecol. Syst. 1996, 27 (1), 365-386.

(13) Hochachka, P. W.; Somero, G. N. Biochemical adaptation : mechanism and process in physiological evolution; Oxford University Press, 2002.

(14) Storey, K. B.; Storey, J. M. EOLSS; Eolss Publishers, 2005; p In: Extremophiles.

(15) Storey, K. B.; Storey, J. M. J. Comp. Physiol. B 1984, 155 (1), 29-36.

(16) Robert Macarthur Award Lecture, T. H.; Henry Wilbur, by M.; Wilbur MacArthur Award Recipient, H. M. Ecology 1997, 78 (8), 2279-2302.

(17) Storey, K. B. Comp. Biochem. Physiol. Part A Physiol. 1997, 117 (3), 319-326. 
(18) Costanzo, J. P.; Lee, R. E.; DeVries, A. L.; Wang, T.; Layne, J. R. FASEB J. 1995, 9 (5), 351-358.

(19) Spector, A. A.; Yorek, M. A. J. Lipid Res. 1985, 26 (9), 1015-1035.

(20) Rodrigues, D. F.; Ivanova, N.; He, Z.; Huebner, M.; Zhou, J.; Tiedje, J. M. BMC Genomics 2008, 9, 547.

(21) Bergholz, P. W.; Bakermans, C.; Tiedje, J. M. J. Bacteriol. 2009, 191 (7), 23402352.

(22) Watson, H. Essays Biochem. 2015, 59, 43-69.

(23) Fan, W.; Evans, R. M. Cell 2015, 161, 962-963.

(24) Cybulski, L. E.; del Solar, G.; Craig, P. O.; Espinosa, M.; de Mendoza, D. J. Biol. Chem. 2004, 279 (38), 39340-39347.

(25) Los, D. A.; Murata, N. Biochim Biophys Acta. 2004, 1666 (1-2), 142-157.

(26) Russell, N. J. In Psychrophiles: from Biodiversity to Biotechnology; Springer Berlin Heidelberg: Berlin, Heidelberg, 2008; pp 177-190.

(27) Matthan, N. R.; Ip, B.; Resteghini, N.; Ausman, L. M.; Lichtenstein, A. H. J. Lipid Res. 2010, 51 (9), 2826-2832.

(28) Zhang, J. Roles of Akt signaling and its downstream pathways in wood frog freeze tolerance Roles of Akt signaling and its downstream pathways in wood frog freeze tolerance, Carleton University, 2013.

(29) Bligh, E. G.; Dyer, W. J. Can. J. Biochem. Physiol. 1959, 37 (8), 911-917.

(30) Benjamini, Y.; Hochberg, Y. Journal of the Royal Statistical Society. Series $B$ (Methodological). WileyRoyal Statistical Society 1995, pp 289-300.

(31) Canez, C. R.; Shields, S. W. J.; Bugno, M.; Wasslen, K. V.; Weinert, H. P.; Willmore, W. G.; Manthorpe, J. M.; Smith, J. C. Anal. Chem. 2016, 88 (14), 69967004.

(32) Chen, X.; Doerge, R. W. 2015, https://arxiv.org/abs/1410.4274v1.

(33) Shaffer, J. P. Annu. Rev. Psychol 1995, 46, 561-584.

(34) McDonald, J. H. Multiple comparisons - Handbook of Biological Statistics, 3rd ed.; Sparky House Publishing: Baltimore, MD, 2014.

(35) Reynolds, A. M.; Lee, R. E.; Costanzo, J. P. J Comp Physiol B 2014, 184, $371-$ 383.

(36) Fajardo, V. A.; McMeekin, L.; LeBlanc, P. J. J. Membr. Biol. 2011, 244 (2), 97103.

(37) Borochov, H.; Zahler, P.; Wilbrandt, W.; Shinitzky, M. Biochim. Biophys. Acta Biomembr. 1977, 470 (3), 382-388. 
(38) Owen, J. S.; Bruckdorfer, K. R.; Day, R. C.; McIntyre, N. J. Lipid Res. 1982, 23 (1), 124-132.

(39) M’Baye, G.; Mély, Y.; Duportail, G.; Klymchenko, A. S. Biophys. J. 2008, 95 (3), 1217-1225.

(40) Cooper, R. A.; Durocher, J. R.; Leslie, M. H. J. Clin. Invest. 1977, 60 (1), 115121.

(41) Slotte, J. P. Prog. Lipid Res. 2013, 52 (4), 424-437.

(42) HIRATA, F.; AXELROD, J. Nature 1978, 275 (5677), 219-220.

(43) Hazel, J. R. Annu. Rev. Physiol. 1995, 57 (1), 19-42.

(44) Tomcala, A.; Tollarova, M.; Overgaard, J.; Simek, P.; Kostal, V. J. Exp. Biol. 2006, 209 (20), 4102-4114.

(45) Dawaliby, R.; Trubbia, C.; Delporte, C.; Noyon, C.; Ruysschaert, J.-M.; Van Antwerpen, P.; Govaerts, C. J. Biol. Chem. 2016, 291 (7), 3658-3667.

(46) Tsakiris, S.; Deliconstantinos, G. Biochem. J 1984, 220, 301-307.

(47) Shivaji, S.; Prakash, J. S. S. Arch. Microbiol. 2010, 192 (2), 85-95.

(48) Ran-Ressler, R. R.; Devapatla, S.; Lawrence, P.; Brenna, J. T. Pediatr. Res. 2008, 64 (6), 605-609.

(49) Broemsen, E.; Doyle, S.; Dieser, M.; Christner, B. In Polar Microbiology: Life in a Deep Freeze; American Society of Microbiology, 2012; pp 103-125. 


\section{Chapter 5. Using MS-based lipidomics to better understand the curious circannual rhythms in goldfish nervous tissue}

\subsection{Abstract}

Goldfish are common model organisms with large retinal horizontal cells ideal for studying neurotransmission. One of the most common methods of studying neurotransmission includes the patch clamp technique. For unknown reasons, however, obtaining data using this technique is difficult during the summer months. One hypothesis for this phenomenon is that the elasticity of retinal membranes differs between the summer and winter months. To investigate this hypothesis, the relative abundances of membrane phospholipids in goldfish retina were measured in January and July to obtain insight into any semiannual phospholipid concentration dynamics that may explain this phenomenon. Performing mass spectrometry-based studies using a hybrid triple quadrupole linear ion trap mass spectrometer revealed a decrease in some of the

phosphatidylethanolamine (PE) species in July. PE is usually linked with the increase of fluidity in biological membranes; fewer PEs, less fluidity. High-resolution mass spectrometry was also performed to determine the identities of the downregulated PEs that may affect the elasticity of goldfish horizontal retinal cell membranes.

\subsection{Introduction}

The goldfish (Carassius auratus) is a freshwater fish that is native to East Asia. They are the most common aquarium fish around the globe. In addition to being a very 
popular pet, goldfish have been of interest to the scientific community for quite some time ${ }^{1-3}$. Goldfish have been used as a model organism to study biology and biochemistry of vision, neuroscience, embryology, as well as behavioral sciences and evolutionary ecology ${ }^{4}$.

A great deal of study on cellular morphology and neurophysiology in sensory systems has been conducted on invertebrates. The most common model organisms in these areas include goldfish, zebrafish (Danio rerio) and larvae of the African clawed frog (Xenopus laevis). These organisms have been used to investigate the role of ion channels in $\mathrm{O}_{2}$ chemoreceptors, the morphology and neurochemistry of chemoreception, and the development of $\mathrm{O}_{2}$-sensing pathways ${ }^{5-7}$. The whole-cell patch clamp method is one of the experimental techniques commonly used to study electrophysiology $y^{8,9}$. The patch clamp technique studies ionic currents in individually isolated living cells, tissue sections, or patches of the cell membrane, so it is possible to identify the neurotransmitter receptors and ion channels ${ }^{10-12}$. The horizontal cells in goldfish retinal tissue are particularly adapted to the patch clamp method, as first demonstrated in 2007 in work by Jonz and Barnes $^{12-15}$. The electrophysiological recordings in this early data were obtained during the winter months; however, recordings during the summer months are limited, as the cells exhibit different physiology. July patch clamp experiments show low voltage as it is more difficult to form an electrical contact on the cells via the small glass recording electrodes. Cellular membranes were observed to have a higher resistance in July compared to in January. This phenomenon is well talked about, but not published, in the electrophysiology community. However, the underlying biomolecular mechanisms for this annular dynamic remain unknown. Here, we investigate the possibility that this seasonal 
change may be due in part to alterations in the membrane lipid composition throughout the year.
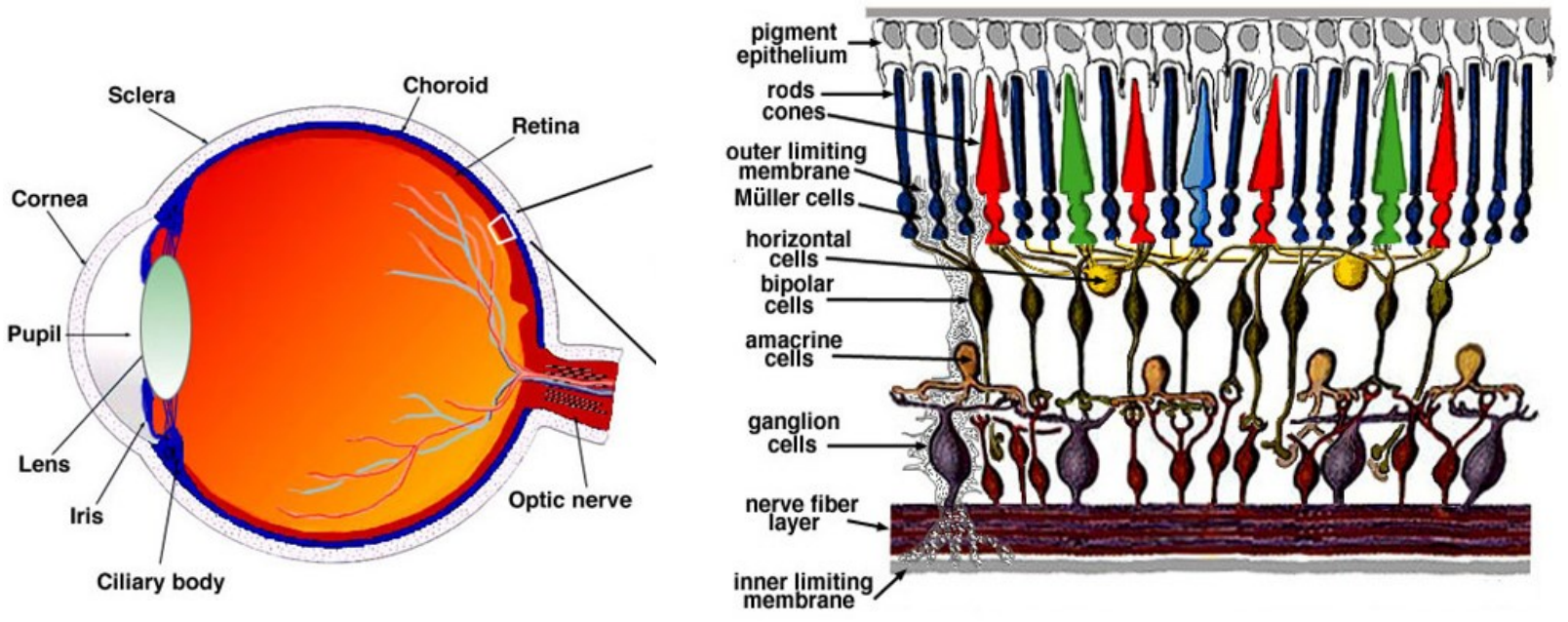

Figure 5:1. Schematic representation of a human eye on the left and enlarged retinal tissue on the right $^{16}$.

Figure 5:1 illustrates a schematic diagram of a human eye and retinal tissue. In the human retina, there are three cones: red, green and blue; goldfish retina also includes an additional cone - ultraviolet. Goldfish are tetrachromat species due to their ability to distinguish between four different primary colors ${ }^{17}$. This capability allows them to have better vision through the surface of the water without troublesome reflections in the water.

Retinal tissue is formed by a complex network of cells, which present a variety shapes and functions. The retina converts light rays into signals that are sent through the optic nerve to the brain where an image is created. Horizontal cells $(\mathrm{HC})$ are lateral elements in retinal tissue, these neurons control signal processing in the outer retina and are themselves under neuromodulatory control from the retina and the brain ${ }^{18}$. HCs are frequently used in membrane electrophysiology studies using the patch clamp 
method. However, the investigation of the individual functions of the retinal tissue cells is outside the scope of this study, instead, intact retinal tissue as a whole has been employed.

Daily physiological rhythms, also called as circadian rhythms, have been extensively studied in fish ${ }^{19-21}$. It has been observed that these rhythms are not passive outcomes of cyclic fluctuations in the environment but originate within the organism. This 24-hour clock is responsible for the time-based organization of many physiological functions such as: sleep, food intake, body temperature, heart rate and hormone release. The retina plays an essential part in organizing the circadian clock; it synchronizes the central clock of the brain with the external day via transduction of the daily light-dark cycle $^{22}$. There is also evidence demonstrating that dysfunction of the circadian rhythms either through genetic mutations or environmental factors initiate the development of various pathologies (e.g. sleep disorders, mood alterations or depression) ${ }^{22}$ or physiological changes. Knowledge of retinal photoreceptors and the discovery of melanopsin (a photopigment belonging to a larger family of light-sensitive retinal proteins) in some ganglion cells have demonstrated that light intensity, timing and spectrum must be considered to keep the biological clock properly functioning ${ }^{23}$. Goldfish retinal circadian rhythms have been studied in relation to food anticipatory activity ${ }^{24-26}$. Melatonin has been widely reported as a hormone involved in regulation of circadian and seasonal rhythms in animals ${ }^{27}$. Annual rhythms like reproduction and hibernation, for example, are also shown by many animals ${ }^{28}$. It is a well-known fact that captive animals do not need to hibernate as there is no need for adaption to a cooler climate. 
The crucian carp (Carassius carassius) and the closely related goldfish can survive for extended periods of time without any oxygen (anoxia), this anoxia tolerance is necessary for fish overwintering at the bottom of ice-covered ponds and lakes. At low or no oxygen levels in the water, fish need to produce adenosine triphosphate (ATP) through anaerobic glycolysis (ATP, is an energy source for organs needed for normal function, without which cells will die). Ethanol is a major end product from this anaerobic glycolysis, which is subsequently released to the water over the gill epithelium. This annual cold avoidance/survival mechanism, however, is lost in fish that are kept in captivity ${ }^{29}$.

This work aims to look for seasonal changes in retinal membrane phospholipid composition and correlate any findings to the annual physiological changes observed in HCs. The phospholipids of interest include phosphatidylcholine (PC), phosphatidylethanolamine (PE), phosphatidylserine (PS) and sphingomyelin (SM) as these lipids are essential components of cell membranes, are found in small concentrations in other parts of the cell and are frequently studied in research examining membrane fluidity ${ }^{30-32}$.

\subsection{Experimental Procedures}

Animals: Adult common goldfish were purchased from AQUAlity Tropical Fish Supply Inc. (Mississauga, ON, Canada) and were housed in the University of Ottawa aquatic facilities. Goldfish were kept in $170 \mathrm{~L}$ aquaria, which were supplied with aerated and dechlorinated $18^{\circ} \mathrm{C}$ water using a flow-through system. Light cycles were maintained at $12 \mathrm{~h}$ light:12 h dark. Animals were killed, and retinas stored in January 2017 (winter) and July 2017 (summer). Protocols for animal care and use were approved by the University 
of Ottawa Animal Care and Veterinary Services (ACVS) protocol (BL-226), which was implemented in accordance with Canadian Council on Animal Care (CCAC) regulations.

Dissection: Removal of retinas closely followed that of Jonz and Barnes, $2007^{12}$. Reagents and chemicals were all purchased from Sigma-Aldrich (Oakville, ON, Canada). Goldfish were adapted to the dark for at least $1 \mathrm{~h}$, euthanized by rapid decapitation and pithed. Eyes were enucleated and immediately moved to cold $\mathrm{Ca}^{2+}$-free Ringer's solution (in mM: $120 \mathrm{NaCl}, 2.6 \mathrm{KCl}, 1 \mathrm{NaHCO}_{3}, 0.5 \mathrm{NaH}_{2} \mathrm{PO}_{4}, 1 \mathrm{Na}$ pyruvate, 4 HEPES, 16 glucose, $\mathrm{pH}$ adjusted to 7.8 with $\mathrm{NaOH}$ ). The eye was cut around the ora serrata to remove the lens, iris, and cornea, and the retina was removed and placed in hyaluronidase (100 U ml-1, Cat. No. H-3506) in L-15 solution for $20 \mathrm{~min}$ at room temperature to break down any retinal vitreous in the preparation. L-15 solution was composed of $70 \% \mathrm{~L}-15$ (Leibovitz's medium without glutamine) and $30 \% \mathrm{Ca}^{2+}$-free Ringer's solution. Retinas were washed 3 times for 3 min each in phosphate buffered saline solution (in mM: $137 \mathrm{NaCl}, 2.7 \mathrm{KCl}, 15.2 \mathrm{NaH}_{2} \mathrm{PO}_{4}, 1.5 \mathrm{KH}_{2} \mathrm{PO}_{4}, \mathrm{pH}$ adjusted to 7.8 with $\mathrm{HCl}$ ), and frozen in this solution with liquid $\mathrm{N}_{2}$.

Two samples were created: one for retina tissue extracted in January 2017 and the other one extracted in July 2017 . Each sample was prepared in the following way: the content of three random tubes, each containing one adult (same age) goldfish retina tissue in phosphate-buffered saline (PBS) solution, were combined to one $15 \mathrm{~mL}$ Corning centrifuge tube. $1 \mathrm{~mL}$ of $2 \%$ acetic acid in methanol $(\mathrm{AcMeOH})$ was added, and the retina samples were sonicated (while still on ice) using a 130-watt ultrasonic processor 
sonicator. Samples were sonicated for 3 minutes with the pulse on for 20 s and pulse off for 10 s using a $50 \%$ amplitude.

Lipid extraction: Lipid extraction was accomplished using a modified Bligh and Dyer method $^{33}$. The samples above were transferred to $10 \mathrm{~mL}$ extraction tubes $(10 \mathrm{~mL}$ glass Kimble tube) with $3.2 \mathrm{~mL}$ of $0.1 \mathrm{M} \mathrm{Na}$ acetate (filtered). The Corning tubes were rinsed 3 times with $1 \mathrm{~mL}$ of $2 \% \mathrm{AcMeOH}$, and each rinsing was transferred to the extraction tube (total volume of $2 \% \mathrm{AcMeOH}$ is $4 \mathrm{~mL}$ ). $60 \mu \mathrm{L}$ of $1 \mathrm{mM}$ internal standard (C13:0 lysophosphatidylcholine) was added to the extraction tubes. Then $3.8 \mathrm{~mL}$ of chloroform was added to the extraction tubes. The extraction tubes were vortexed, swirled and inverted 3 times and placed in a centrifuge for 2 minutes at $2000 \mathrm{rpm}$ and $4^{\circ} \mathrm{C}$. The bottom phase was collected with a Pasteur pipette and transferred to collection tubes (10 $\mathrm{mL}$ glass Kimble tubes). Extractions were repeated 3 more times with $2 \mathrm{~mL}$ of chloroform each time, all of the bottom phases were retained in the same collection tubes for each sample. Then all of the chloroform was evaporated under a constant stream of nitrogen gas until a dark yellow oily residue remained. The retina oil samples were resuspended in $600 \mu \mathrm{L}$ of $10 \mathrm{mM}$ ammonium acetate in absolute $\mathrm{EtOH}$. Then samples were incubated at $30^{\circ} \mathrm{C}$ for 10 minutes to dissolve lipids and centrifuged for 1 minute at $2000 \mathrm{rpm}$ and $4^{\circ} \mathrm{C}$. The samples were diluted 40 times in $10 \mathrm{mM}$ ammonium acetate in absolute $\mathrm{EtOH}$ and then transferred to an amber glass tube, flushed with $\mathrm{N}_{2}$ gas and stored in a $-20^{\circ} \mathrm{C}$ freezer until analysis if analyzed within a week; samples requiring longer storage times were frozen at $-80^{\circ} \mathrm{C}$.

HPLC-ESI-MS/MS analysis: $88 \mu \mathrm{L}$ of Kasil\#1 was mixed with $16 \mu \mathrm{L}$ of formamide in an Eppendorf tube and vortexed for two minutes. Approximately $15 \mathrm{~cm}$ long pieces of fused 
silica tubing with inner diameters of $200 \mu \mathrm{m}$ were dipped into the Kasil/formamide mixture; silica tubes were left overnight to dry allowing frit to polymerize (dipped tip facing downwards). These fritted columns were packed with $5.5 \mathrm{~cm}$ of C4-bonded silica using a nitrogen pressure vessel and were then fritted on the other end as well, as described above. These columns were used for reversed phase chromatography to separate the complex lipid samples.

The following HPLC solvents were used: solvent A (10 mM ammonium acetate in $30 \%$ methanol/70\% MilliQ water), solvent B (10 mM ammonium acetate in $75 \%$ isopropanol (IPA)/25\% methanol), and solvent D (methyl tert-butyl ether (MTBE)). The gradient began at $100 \%$ solvent A and gradually increased to $100 \%$ solvent B in 41.4 minutes, the run ended in MTBE and by 48 minutes it was at $100 \%$ solvent $D$ (total run time was 60 minutes).

The sample mixture for separation and analysis on the HPLC-ESI-MS was prepared by using $12.5 \mu \mathrm{L}$ of the diluted extracted lipid sample and $37.5 \mu \mathrm{L}$ of $10 \mathrm{mM}$ ammonium acetate in MilliQ water. The sample was injected into the column using an HPLC (Dionex Ultimate 3000) auto-sampler. The sample was introduced into the hybrid triple quadrupole linear ion trap mass spectrometer (AB Sciex QTRAP 4000, AB Sciex, Framingham, MA) using an electrospray ionization source with an electrode with an inner diameter of $100 \mu \mathrm{m}$.

A precursor ion scan for $\mathrm{m} / \mathrm{z} 184$ at low resolution was used to identify and quantify lipids with a PC head group. The low-resolution scan was set with a mass range of 400 to 1000 Th. A precursor ion scan for $\mathrm{m} / \mathrm{z} 184$ at unit resolution was done for SM identification and quantification to differentiate between PCs and SMs; the range of this 
scan was from 600 to 900 Th. At low resolution, neutral loss scans for $141 \mathrm{Da}$ and 185 Da were used to identify and quantify PE and PS, respectively. The neutral loss scan for PE had a range of 300 to 900 Th while the neutral loss scan for PS had a range of 400 to 1100 Th. All scans were conducted in positive ion mode, and triplicate analyses were done for both the January and July samples.

LC/MS and LC/MS/MS high-resolution experiments were carried out on an Agilent 6550 Q-TOF equipped with iFunnel technology. Agilent's branded iFunnel technology joins the high-efficiency ESI ion generation and focusing of Agilent Jet Stream sample introduction with unique hexabore sampling capillary and dual stage ion funnel assemblies $^{34}$. The 6550 Q-TOF instrument has shown double-digit increases in sensitivity compared to older instruments. The following HPLC solvents were used: solvent A (10 $\mathrm{mM}$ ammonium acetate in $30 \%$ methanol/70\% MilliQ water), solvent $\mathrm{B}$ (10 mM ammonium acetate in $75 \%$ IPA/25\% methanol). The instrument was run in positive ion mode for high mass accuracy identification and negative ion mode for fatty acid identification.

Selective functional group derivatization reactions: To identify vinyl-ether containing lipids from alkyl-ether containing lipids, lipids were reacted with formic acid as described by Fhaner et la., $2012^{35}$. This simple one-minute reaction hydrolyzes all plasmalogens (lipids with the vinyl-ether group) in the sample. After formic acid reactions, lipids were redissolved in $10 \mathrm{Mm}$ ammonium acetate in absolute $\mathrm{EtOH}$ for MS analysis.

Data analysis and statistical analysis: Analyst 1.5 .1 software was used to extract peak list data from the chromatograms. The extracted peak lists were entered into the MultiQaunt 2.1.1 software to obtain peak area values. Data standardization was done in 
two different ways: first, the January tissue total area value and the July tissue total area value were standardized to each other, and all of the peak areas were standardized to the area of the internal standard. Averages of all of the peaks were calculated with their standard deviation values. To be able to identify the significant changes between the January and July lipid abundances, lipid fold changes were calculated, and a student's ttest was performed $(\alpha=0.05)$. To control the false discovery rate, the Benjamini-Hochberg $(\mathrm{BH})$ method was performed with $\alpha=0.15^{36}$, and $p$-values from the student's t-test and $\mathrm{BH}$ methods were compared. High-resolution MS data were analyzed using MassHunter Qualitative Analysis B.07.00 software. The lipid database Lipidomics Gateway at (www.lipidmaps.org) was used for lipid identification. ChemDraw Ultra 12.0 software was used to draw glycerophospholipid structures.

\subsection{Results}

Analysis of the low-resolution MS data yielded a total of 153 glycerophospholipids and sphingomyelins in goldfish retinal tissue that were above the limit of quantification (LOQ). The relative amounts of each class of lipid included 90 PCs, 37 PEs, 7 PSs and 19 SMs (Figure 5:2). The LOQ has been calculated by established protocols for the instrument used in the experiments ${ }^{37}$.

Phospholipid concentration dynamics between January and July goldfish retinal samples were investigated to determine if they play a role in horizontal cell membrane elasticity. This was done by looking at the standardized area values of lipids found from 


\section{Phospholipids (153) in goldfish eye retina}

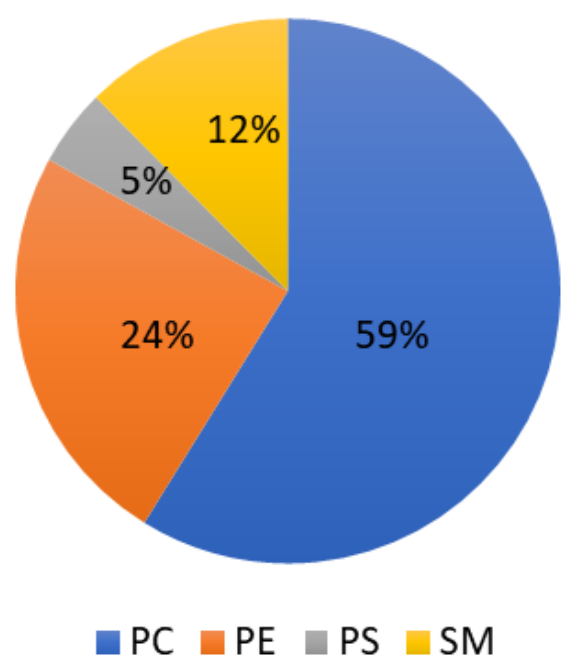

Figure 5:2. Relative abundances of glycerophospholipids and sphingomyelins found in goldfish eye retina samples. There are a total of 153 phospholipids found in goldfish eye retina samples above the limit of quantification (LOQ).

MS data and calculating $p$-values $(\alpha=0.05)$ using a t-test to elucidate any significant concentration changes. It was found that $88 \%$ of phospholipids in the goldfish retina sample do not go through a significant concentration change in July compared to January (Figure 5:3). The other phospholipids (12\%) were found to change concentration in a statistically significant manner, with some decreasing and others increasing during July compared to January. A correction for false discovery rate (FDR) was also performed using the Benjamini-Hochberg $(\mathrm{BH})$ method with an $\alpha=0.15$. This method was chosen as it appears to be the most optimal for the study performed, the traditional Bonferroni correction is too strict and may introduce false negative errors ${ }^{38,39}$. The bottom chart in Figure 5:3 shows significant phospholipid concentration changes in the goldfish retina membranes after the $\mathrm{BH}$ method correction. The number of significantly changing species has decreased compared to the t-test value alone from $12 \%$ to $3 \%$. 


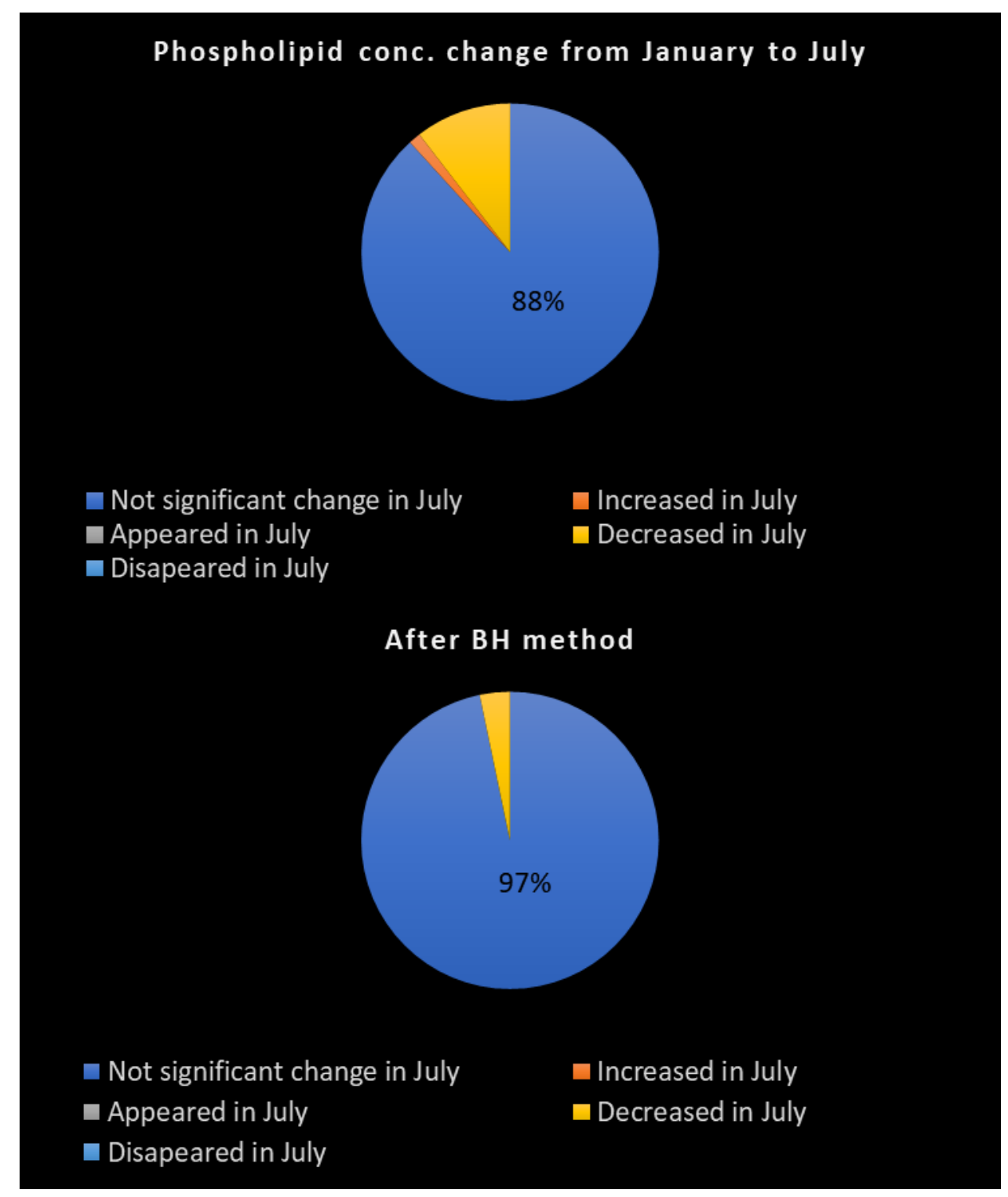

Figure 5:3. Phospholipid concentrations are compared between January and July goldfish eye retina tissues. The phospholipid concentration could be not changing, increasing/decreasing, appearing/disappearing in the July tissue when compared to the January tissue. The bottom panel shows the same comparison after a false discovery rate correction using the Benjamini-Hochberg (BH) method $(\alpha=0.15)$.

The $3 \%$ of phospholipids that were found to decrease by approximately $50 \%$ in July compared to January were all found to be PEs and are summarized in Table 5:1. The possible structures of these lipids derived from the lipid database on Lipidomics 
Gateway are also indicated based on the nominal mass of each lipid derived from lowresolution MS experiments. To narrow down structural possibilities and find the true identities of lipids that are downregulated in the summer months, high-resolution MS data was collected, and fatty acids fragments were identified for 5 significant lipids found. To resolve these isobaric issues further, formic acid hydrolysis was performed by established methods ${ }^{35}$. This procedure hydrolyzes plasmalogen species and allows one to distinguish vinyl-ether containing lipids from alkyl-ether containing lipids.

Table 5:1. Significant PEs that decrease during the seasonal change from January to July.

\begin{tabular}{cccc}
\hline $\begin{array}{c}\text { Phospholipid } \\
\text { class }\end{array}$ & $\begin{array}{c}\text { Low-resolution } \\
\text { masses of } \\
\text { phospholipids }(\mathrm{m} / \mathrm{z})\end{array}$ & $\begin{array}{c}\text { Possible structures of } \\
\text { phospholipids }\end{array}$ & Retention time (min) \\
\hline PE & 688 & PE(32:2), PE(O-33:2), PE(P-33:1) & $26.2 / 27.8$ \\
\hline PE & 704 & PE(33:1), PE(O-34:1), PE(P-34:0) & $25.8 / 27.6$ \\
\hline PE & 730 & PE(35:2), PE(O-36:2), PE(P-36:1) & $26.1 / 28.9$ \\
\hline PE & 738 & PE(36:5) & 26.8 \\
\hline PE & 820 & PE(42:6) & 27.7 \\
\hline
\end{tabular}

Figure 5:4 illustrates chromatograms obtained with a high-resolution mass spectrometer and the exact masses of the significant PEs that change in concentration during the summer months compared to winter months in goldfish retina membranes. The PEs shown in Figure 5:4 are the diacyl species (both fatty acid groups are linked to glycerol via an ester bond). High-resolution data distinguishes between diacyl species and plasmalogen/ethereal species. However, due to the complexity of the biological samples, various phospholipids are found in the samples and very often one nominal mass is a combination of multiple diacyl phospholipids, ethereal-acyl phospholipids and/or plasmalogen-acyl phospholipids. 


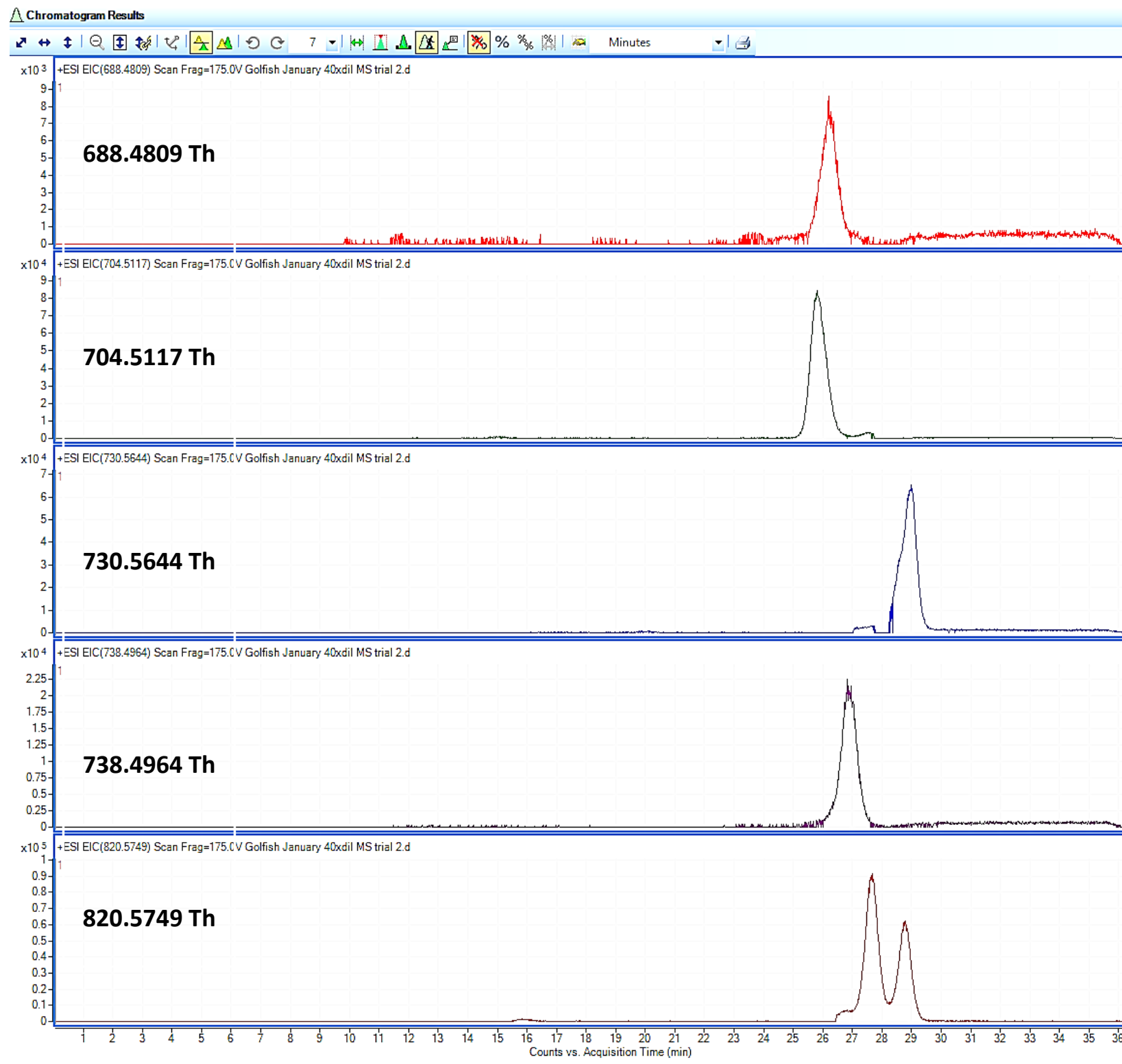

Figure 5:4. High-resolution MS extracted ion chromatograms from five PEs that decrease in concentration in summer months compared to winter months in goldfish retinal tissue. These chromatograms allow for the determination of the exact masses of the PEs of interest (indicated in the title of each chromatogram).

Negative ion MS/MS data allows for the identification of the fatty acyl groups. The PEs that changed in concentration between the seasons in goldfish retinal membranes were again examined in fatty acid fragmentation experiments to identify fatty acyl chains that were found in combination with exact masses of the PEs of interest. This data 
narrowed down the possible structures of the PEs of interest, and the results are summarized in Table 5:2.

Table 5:2. Identified PEs with exact mass data and relative abundances.

RELATIVE ABUNDANCE

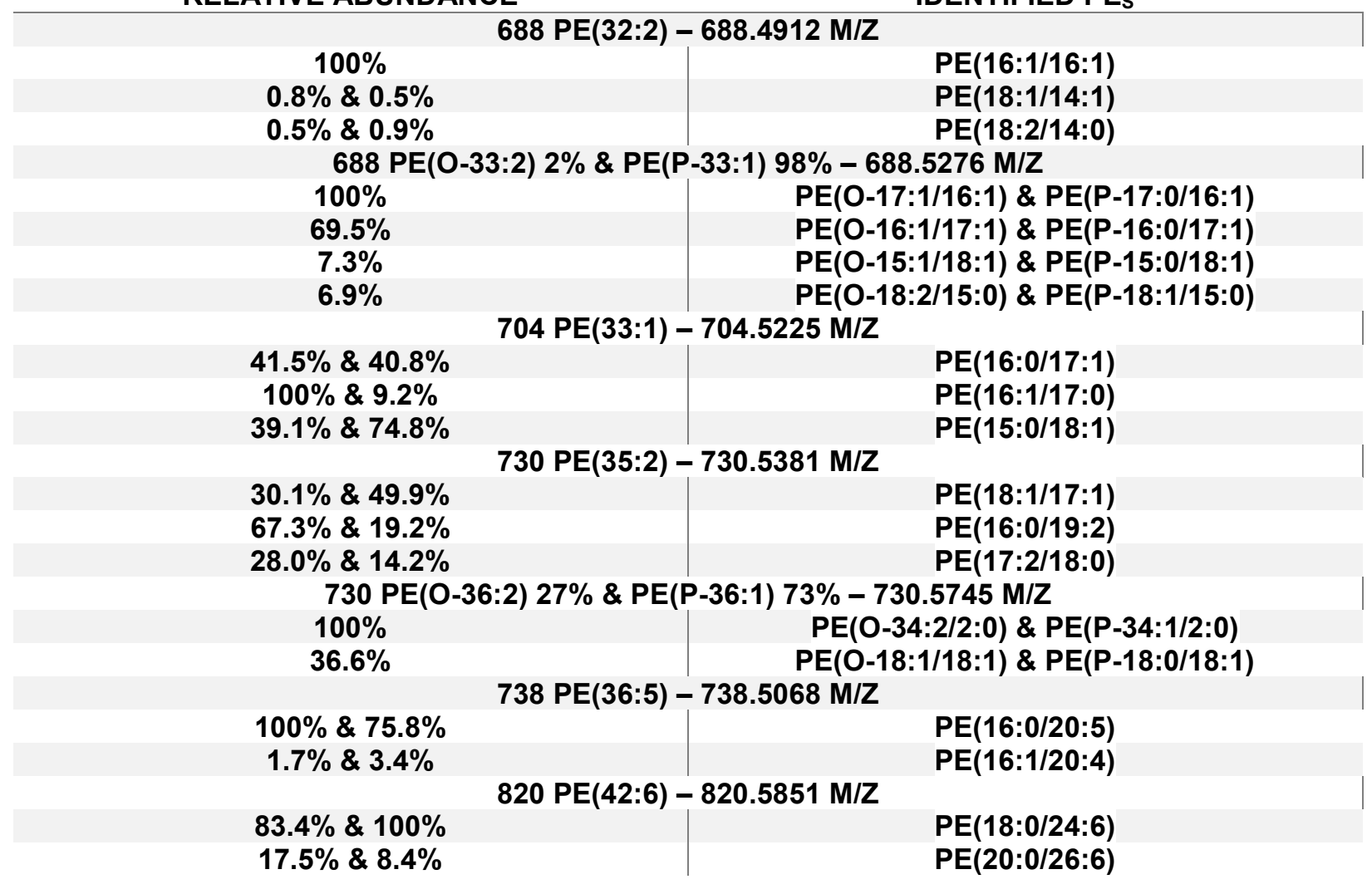

PE species are identified by their relative abundances at each exact mass at a time. For $\mathrm{m} / \mathrm{z} 688 \mathrm{PE}$, two exact masses could be noted in table 5:2: 688.4912 and 688.5276, which indicates that a diacyl species as well as an ethereal-acyl and/or plasmalogen-acyl species are present in the sample for that nominal mass. Both of these exact masses were further examined MS/MS experiments. The relative abundances of the fatty acids chains were found for every exact mass. For $\mathrm{m} / \mathrm{z} 688.4912 \mathrm{PE}$, the most abundant fatty acid chain was found to be 16:1 (16 carbons with one double bond fatty acid, $\left.\mathrm{CH}_{3} \mathrm{C}_{14} \mathrm{H}_{26} \mathrm{COO}^{-}\right)$. The most abundant $\mathrm{MS} / \mathrm{MS}$ peak would be noted as $100 \%$, and 
the rest of the MS/MS peaks would be relative to that $100 \%$ peak. Since other fatty acids are in such a low abundance for $\mathrm{m} / \mathrm{z} 688.4912$, it can be concluded that $P E$ at $\mathrm{m} / \mathrm{z}$ 688.4912 is mostly diacyl $\operatorname{PE}(16: 1 / 16: 1)$. The locations of the double bonds are not known; however, the lipid database Lipidomics Gateway always shows that two fatty acids chains have double bonds in the same location. The $\mathrm{m} / \mathrm{z} 688.5276$ PE is either an ethereal-acyl phospholipid or a plasmalogen-acyl phospholipid or a combination of both. After formic acid hydrolysis, it was observed that the $\mathrm{m} / \mathrm{z} 688.5276$ peak was reduced by $98 \%$. Since this experiment hydrolyzes the plasmalogen-acyl phospholipids, the $\mathrm{m} / \mathrm{z}$ 688.5276 peak is $98 \%$ plasmalogen-acyl phospholipid and $2 \%$ ethereal-acyl phospholipid. Vinyl-ether and alkyl-ether fatty acids do not fragment in MS/MS to reveal the alkyl groups; only acyl fatty acids could be identified via these experiments. Vinylether or alkyl-ether fatty acids are generally calculated via the difference between the exact mass of the intact lipid and the acyl fatty acid. For $\mathrm{m} / \mathrm{z} 688.5276 \mathrm{PE}$, the most abundant fatty acids are $16: 1100 \%$, followed by $17: 169.5 \%$. Figure $5: 5$ shows the most likely structures for lipids with a nominal mass of $\mathrm{m} / \mathrm{z} 688$; the most abundant peaks found through experiment and most common peaks found in the lipid database Lipidomics Gateway.

The structures for the other four significant PE were identified by similar processes. The $\mathrm{m} / \mathrm{z} 704$ was concluded to be only diacyl species peak as there were no peaks found for plasmalogen-acyl/ ethereal-acyl species on the high-resolution MS. This PE has many possible fatty acid chains; the three most abundant structures found for this mass are $\operatorname{PE}(16: 1 / 17: 0), \operatorname{PE}(15: 0 / 18: 1)$ and $\mathrm{PE}(16: 0 / 17: 1)$. There are diacyl species as well as 


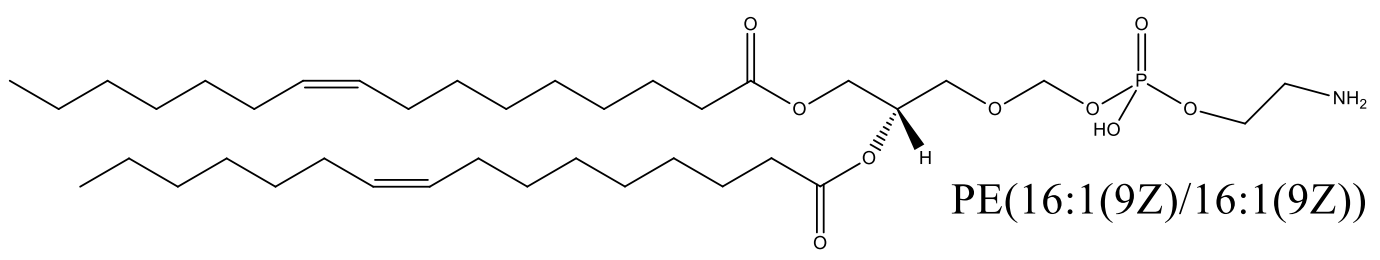

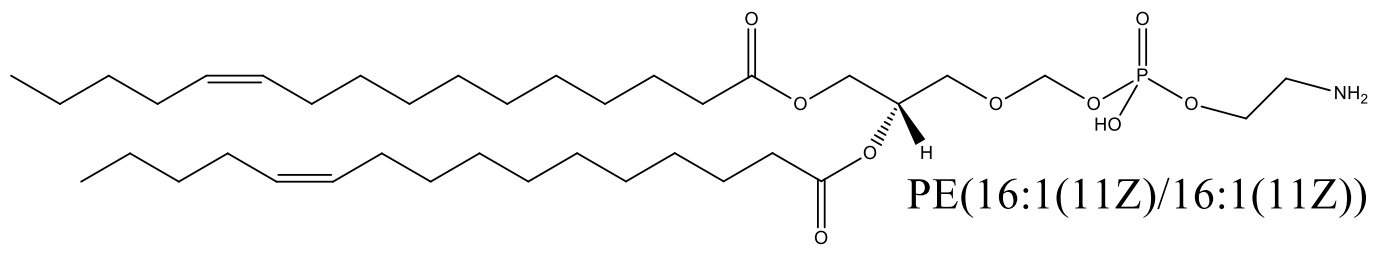

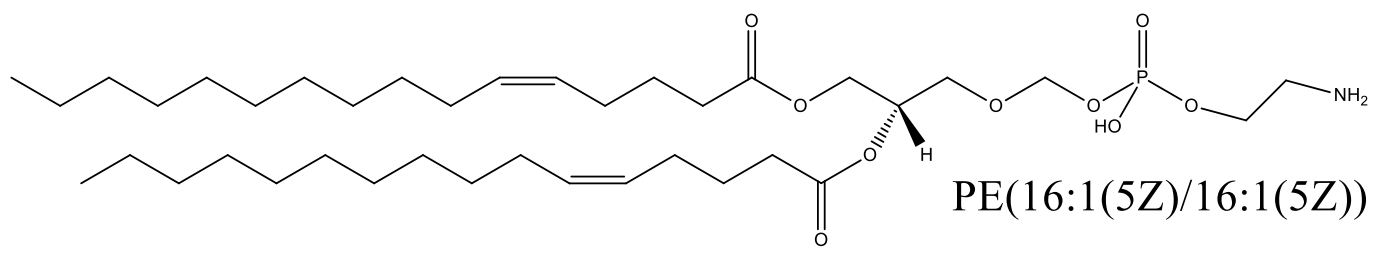<smiles>[2H][C@@](COCOP(=O)(OCC)OCCN)(COC(=O)CCCCCCC/C=C\CCCCCCCC)CC(=O)OCC</smiles><smiles>CCCCC/C=C\C/C=C\CCCCCCCC(=O)OC[C@](C)(COCOP(=O)(OCC)OCCN)OC(=O)CCCCCCCCCC</smiles><smiles>[2H][C@H](CO/C=C\CCCCCCCCCCCCCC)COCOP(=O)(OCCN)OCCCCCCCCCCCCC</smiles>

Figure 5:5. Possible structures of nominal mass $m / z 688$ PEs after fatty acid chain identification and determination of relative proportions of ethereal-acyl to plasmalogen-acyl species. These PE structures are also found in the lipid database of Lipidomics Gateway. Many other possible structures are possible; this Figure does not consider sn-1 and sn-2 locations of the fatty acid chains as well the location and the configuration of the double bonds (though $Z$ configurations of the double bond are the most common ones) 
ethereal-acyl and/or plasmalogen-acyl species present in the sample for nominal $\mathrm{m} / \mathrm{z} 730$. The most abundant diacyl species for this mass are $P E(16: 0 / 19: 2)$ and $P E(18: 1 / 17: 1)$, and most abundant ethereal-acyl and plasmalogen-acyl species are 27\% PE(O-34:2/2:0) and $73 \% \mathrm{PE}(\mathrm{P}-34: 1 / 2: 0)$. Lastly, $\mathrm{m} / \mathrm{z} 738$ is mostly identified as $\mathrm{PE}(16: 0 / 20: 5)$, and $\mathrm{m} / \mathrm{z}$ 820 is mostly identified as $\mathrm{PE}(18: 0 / 24: 6)$. Other examples of possible structural combinations could be found in table 5:2.

\subsection{Discussion}

To date, a minimal amount of scientific literature is available on goldfish retinal phospholipid class distribution and dynamics. However, the data observed in this study is consistent with the study reported by Fliesler et al., 1983. They found the following distribution of lipids in goldfish retina in relative mol\%: 57.7 PC, 32.7 PE, 5.9 PS, 1.5 SM, 1.0 phosphatidylinositol $(\mathrm{Pl})$ and 1.3 unknown $^{40}$. Fliesler et al., 1983 analyzed phospholipids by two-dimensional thin-layer chromatography (2D TLC) by methods described by Anderson et al., 197041. Fliesler et al., 1983 showed higher PE amounts ( $33 \%$ vs. $24 \%)$ and lower SM amounts ( $2 \%$ vs. $12 \%)$ in the goldfish retina compared to this study. Fliesler et al., 1983 used 2D TLC for their lipid analysis which was historically a method of choice for lipid research as it allowed for rapid screening of lipid extracts and did not require any sophisticated instrumentation. The TLC method, however, exposes samples to air for prolonged periods of time which can lead to lipid degradation. Also, the

TLC method is quite time-consuming and lacks resolution power and specificity ${ }^{42}$. Most mammalian tissues have SM proportions between 2 to $15 \%$, with higher concentrations of SM found in nerve tissues ${ }^{43,44}$. The retina tissue is an extension of the brain and 
contains light-sensitive photoreceptor cells; which does not contradict the higher observed SM concentration in this study compared to Fliesler et al., 1983.

Cellular membrane fluidity is studied extensively in animals that adapt to cooler temperatures for overwintering ${ }^{32,45}$ and membrane fluidity has been examined in various disease studies ${ }^{46-48}$. The fluidity of membranes is known to be regulated by fatty acid desaturases ${ }^{49,50}$ and cholesterol ${ }^{51,52}$. Unfortunately, investigating cholesterol dynamics was beyond the scope of this work. This study was designed to look at the major glycerophospholipid components of cell membranes which all affect membrane fluidity and elasticity ${ }^{53}$. In this study it was found that $3 \%$ of PCs, PEs, PSs and SMs that were above the LOQ change in concentration between seasons. This small percentage makes it difficult to comment on the role of desaturases in this physiological phenomenon that is observed in goldfish retinal tissue.

There were no new phospholipids identified between the seasons. However, it is important to note that all significant phospholipid concentration changes came from PE species in this study. Another way to regulate membrane fluidity is through the relative ratios of the different glycerophospholipid subclasses investigated in this study. PE is considered a "disordering lipid" due to its conical shape which decreases the degree of close packing of lipids compared to other phospholipds ${ }^{32}$. The increase of PE is associated with increase of fluidity ${ }^{54,55}$ and is considered as the key regulator in membrane fluidity in eukaryotic cells ${ }^{56}$. On the contrary, the decrease of PEs can be associated with the decrease of fluidity of cell membranes. This fluidity decrease via lower PE levels may be responsible for making the retinal membranes more rigid during the summer time period. The seasonal variation in retinal cell membrane phospholipid 
structure in captive goldfish is not extensively large; however, the trend of differing retinal cell membrane fluidity/rigidity is consistent in goldfish at each time of the year.

\subsection{Conclusion}

It is important to note that many captive animals lose their need to hibernate or prepare themselves for lower temperature survival and adoption to a colder climate. However, many species also maintain their circadian and annual physiological rhythms for healthy living. The annual rhythm in goldfish retina tissue is preserved in captivity as observed from differences in electrophysiology in relation to the summer and winter months. It has been observed that goldfish retina cell membranes decrease their PE concentrations in summer months. One possible explanation for this is that historically, the winter months involve colder temperatures, requiring membrane fluidity to increase to maintain cellular homeostasis. In contrast, the summer months, with warmer temperatures, require less membrane fluidity and the goldfish have adapted to this annual rhythm by controlling the relative concentration of $P E$ in their nervous tissue. The decreased fluidity in summer months correlates with increased membrane stiffness and may be one of the reasons that retinal horizontal cell patch-clamp studies are difficult to conduct during summer months. 


\subsection{Acknowledgments}

The tissue for this study came from the University of Ottawa and was extracted by Michael Country. The financial support from NSERC for this work is much acknowledged by MJ and JCS.

\subsection{References}

(1) Smith, G. M.; Burr, H. S.; Ferguson, R. S. Endocrinology 1935, 19 (4), 409-412.

(2) Caldwell, R. S. Comp. Biochem. Physiol. 1969, 31 (1), 79-93.

(3) Zhang, J.; Shen, H.; Wang, X.; Wu, J.; Xue, Y. Chemosphere 2004, 55 (2), 167174.

(4) Encyclopedia of fish physiology: from genome to environment., Volume 1.; Farrell, A. P., Cech, J. J., Richards, J. G., Stevens, E. D., Eds.; Elsevier, 2011.

(5) Jonz, M. G.; Fearon, I. M.; Nurse, C. A. J. Physiol. 2004, 560 (Pt 3), 737-752.

(6) Burggren, W. W.; Warburton, S. ILAR J. 2007, 48 (3), 260-269.

(7) Zachar, P. C.; Pan, W.; Jonz, M. G. J. Neurophysiol. 2017, 118 (6), 3014-3023.

(8) Lado, W. E.; Spanswick, D. C.; Lewis, J. E.; Trudeau, V. L. Front. Neurosci. 2014, 8, 185.

(9) Zhang, D. Evid. Based. Complement. Alternat. Med. 2012, 2012, 534219.

(10) Kaneko, A.; Tachibana, M. J. Physiol. 1985, 358, 131-152.

(11) Fohlmeister, J. F.; Miller, R. F. J. Neurophysiol. 1997, 78 (4), 1935-1947.

(12) Jonz, M. G.; Barnes, S. J. Physiol. 2007, 581 (Pt 2), 529-541.

(13) Paik, S.-S.; Bai, S.-H.; Jung, C.-S. Korean J. Physiol. Pharmacol. 2005, 9 (5), 269-273.

(14) Vanleeuwen, M.; Fahrenfort, I.; Sjoerdsma, T.; Numan, R.; Kamermans, M. J. Neurosci. 2009, 29 (19), 6358-6366.

(15) Vroman, R.; Klaassen, L. J.; Howlett, M. H. C.; Cenedese, V.; Klooster, J.; Sjoerdsma, T.; Kamermans, M. PLoS Biol 2014, 12 (5).

(16) Kolb, H. Simple Anatomy of the Retina by Helga Kolb - Webvision http://webvision.med.utah.edu/book/part-i-foundations/simple-anatomy-of-the- 
retina/ (accessed Feb 20, 2018).

(17) Neumeyer, C.; Arnold, K. Vision Res. 1989, 29 (12), 1719-1727.

(18) Thoreson, W. B.; Mangel, S. C. Prog. Retin. Eye Res. 2012, 31 (5), 407-441.

(19) Reiter, R.; Tan, D.-X.; Manchester, L. In Biological Clock in Fish; Science Publishers, 2010; pp 71-91.

(20) Lahiri, K.; Foulkes, N.; Manchester, L. C. In Biological Clock in Fish; Science Publishers, 2010; pp 93-110.

(21) luvone, P.; Velarde, E.; Delgado, M.; Alonso-Gomez, A.; Haque, R. In Biological Clock in Fish; Science Publishers, 2010; pp 251-259.

(22) Springer Series in Vision Research The Retina and Circadian Rhythms; Tosini, G., luvone, P. M., McMahon, D. G., Collin, S. P., Eds.; Springer International Publishing: New York, 2014.

(23) Bonmati-Carrion, M. A.; Arguelles-Prieto, R.; Martinez-Madrid, M. J.; Reiter, R.; Hardeland, R.; Rol, M. A.; Madrid, J. A. Int. J. Mol. Sci. 2014, 15 (12), 2344823500.

(24) Aranda, A.; Madrid, J. A.; Sánchez-Vázquez, F. J. J. Biol. Rhythms 2001, 16 (1), 50-57.

(25) Vera, L. M.; De Pedro, N.; Gómez-Milán, E.; Delgado, M. J.; Sánchez-Muros, M. J.; Madrid, J. A.; Sánchez-Vázquez, F. J. Physiol. Behav. 2007, 90 (2-3), 518524.

(26) Velarde, E.; Haque, R.; luvone, P. M.; Azpeleta, C.; Alonso-Gómez, A. L.; Delgado, M. J. J Biol Rhythm. 2009, 24 (2), 104-113.

(27) Reiter, R. J. Experientia 1993, 49 (8), 654-664.

(28) Biological clock in fish; Kulczykowska, E., Popek, W., Kapoor, B. G., Eds.; Science Publishers: Enfield, 2010.

(29) Encyclopedia of fish physiology: from genome to environment., Volume 3.; Farrell, A. P., Stevens, E. D., Cech, J. J., Richards, J. G., Eds.; Elsevier, 2011.

(30) Borochov, H.; Zahler, P.; Wilbrandt, W.; Shinitzky, M. Biochim. Biophys. Acta Biomembr. 1977, 470 (3), 382-388.

(31) Fajardo, V. A.; McMeekin, L.; LeBlanc, P. J. J. Membr. Biol. 2011, 244 (2), 97103.

(32) Reynolds, A. M.; Lee, R. E.; Costanzo, J. P. J Comp Physiol B 2014, 184, $371-$ 383.

(33) Bligh, E. G.; Dyer, W. J. Can. J. Biochem. Physiol. 1959, 37 (8), 911-917.

(34) Breakthrough iFunnel Technology for CLEARLY BETTER sensitivity, 2017. 
(35) Fhaner, C. J.; Liu, S.; Ji, H.; Simpson, R. J.; Reid, G. E. Anal. Chem. 2012, 84 (21), 8917-8926.

(36) Benjamini, Y.; Hochberg, Y. Journal of the Royal Statistical Society. Series $B$ (Methodological). WileyRoyal Statistical Society 1995, pp 289-300.

(37) Canez, C. R.; Shields, S. W. J.; Bugno, M.; Wasslen, K. V.; Weinert, H. P.; Willmore, W. G.; Manthorpe, J. M.; Smith, J. C. Anal. Chem. 2016, 88 (14), 69967004.

(38) Noble, W. S. Nat. Biotechnol. 2009, 27 (12), 1135.

(39) Waite, T.; Campbell, L. Écoscience 2006, 13 (4), 439-442.

(40) Fliesler, S. J.; Maude, M. B.; Anderson, R. E. Biochim. Biophys. A cta 1983, 734, 144-152.

(41) Anderson, R. E.; Feldman, L. S.; Feldman, G. L. Biochim. Biophys. Acta 1970, 202, 367-373.

(42) Khalil, M. B.; Hou, W.; Zhou, H.; Elisma, F.; Swayne, L. A.; Blanchard, A. P.; Yao, Z.; Bennett, S. A. L.; Figeys, D. Mass Spectrom. Rev. 2010, 29, 877-929.

(43) Ramstedt, B.; Slotte, J. P. FEBS Lett. 2002, 531 (1), 33-37.

(44) Koval, M.; Pagano, R. E. Biochim. Biophys. Acta 1991, 1082 (2), 113-125.

(45) Rodriguez-Vargas, S.; Sanchez-Garcia, A.; Martinez-Rivas, J. M.; Prieto, J. A.; Randez-Gil, F. Appl. Environ. Microbiol. 2007, 73 (1), 110-116.

(46) Mathew, B.; Srinivasan, K.; Pradeep, J.; Thomas, T.; Mandal, A. K. Asian J. Psychiatr. 2018, 32, 105-109.

(47) Batta, G.; Soltész, L.; Kovács, T.; Bozó, T.; Mészár, Z.; Kellermayer, M.; Szöllősi, J.; Nagy, P. Sci. Rep. 2018, 8 (1), 157.

(48) Sameni, S.; Malacrida, L.; Tan, Z.; Digman, M. A. Sci. Rep. 2018, 8 (1), 734.

(49) Maulucci, G.; Cohen, O.; Daniel, B.; Sansone, A.; Petropoulou, P. I.; Filou, S.; Spyridonidis, A.; Pani, G.; De Spirito, M.; Chatgilialoglu, C.; Ferreri, C.; Kypreos, K. E.; Sasson, S. Free Radic. Res. 2016, 50 (sup1), S40-S50.

(50) Ballweg, S.; Ernst, R. Biol. Chem. 2017, 398 (2), 215-228.

(51) Subczynski, W. K.; Pasenkiewicz-Gierula, M.; Widomska, J.; Mainali, L.; Raguz, M. Cell Biochem. Biophys. 2017, 75 (3-4), 369-385.

(52) Kaddah, S.; Khreich, N.; Kaddah, F.; Charcosset, C.; Greige-Gerges, H. Food Chem. Toxicol. 2018, 113, 40-48.

(53) Lodish, H.; Berk, A.; Zipursky, S. In Molecular Cell Biology; Freeman, W., Ed.; New York, 2000.

(54) Guti6rrez-Ruiz, M. C.; G6mez, J. L.; Souza, V.; Bucio, L. Cell Bioiogy Toxicol. 
1995, 11, 69-78.

(55) Pollard, A. K.; Ortori, C. A.; Stöger, R.; Barrett, D. A.; Chakrabarti, L. Aging (Albany. NY). 2017, 9 (3), 986-998.

(56) Dawaliby, R.; Trubbia, C.; Delporte, C.; Noyon, C.; Ruysschaert, J.-M.; Van Antwerpen, P.; Govaerts, C. J. Biol. Chem. 2016, 291 (7), 3658-3667. 


\section{Chapter 6. The analysis of lipidomic data}

\subsection{Abstract}

The purpose of this chapter is to describe the lipidomics data processing that was done on all of the lipidomics projects in this thesis. The thirteen-lined ground squirrel hibernation project was a collaborative project between quite a few members of our group. From the hibernation project, there was enough interest generated to continue research into cell membrane phospholipids in freeze tolerance using a wood frog as a study model. The wood frog project investigated freeze tolerance in a vital organ versus a non-vital organ (liver versus leg muscle). The wood frog project investigated patterns obtained from low-resolution mass spectrometry (MS) data using the hybrid triple quadrupole linear ion trap mass spectrometer (AB Sciex QTRAP 4000). The purpose of that project was to look at the changes in cell membrane fluidity in different seasons. The goldfish project was investigated freeze avoidance patterns obtained from goldfish retina tissue membranes. After finding the initial pattern in retinal membrane fluidity, it was of interest to look at specific phospholipids that change significantly between the seasons using highresolution mass spectrometry instrument (Agilent iFunnel 6550 Q-TOF). The whole workflow of data processing is shown here using goldfish retina membranes phospholipid data as it includes low-resolution and high-resolution mass spectrometric data. The workflow only includes data processing after data collection, which was done mostly in Excel 2016; however, data extraction will be shown as well. 


\subsection{The workflow}

Low-resolution data: The data was collected with the hybrid triple quadrupole linear ion trap mass spectrometer (AB Sciex QTRAP 4000) which is connected to high-performance liquid chromatography (HPLC) for separations. Data collection was done in triplicates and yielded .wiff extension files. The data on the QTRAP instrument was collected into total ion current (TIC) versus time chromatogram, however, since it is ion trap instrument, the phospholipid classes can be distinguished from each other, and four data sets were collected depending on the head group of the phospholipid class onto one TIC chromatogram. PC and SM fragment to form an ion of $\mathrm{m} / \mathrm{z} 184$, PE has neutral loss of $141 \mathrm{Da}$ and PS has neutral loss of $185 \mathrm{Da}$. These fragments are shown in Figure 6:1.

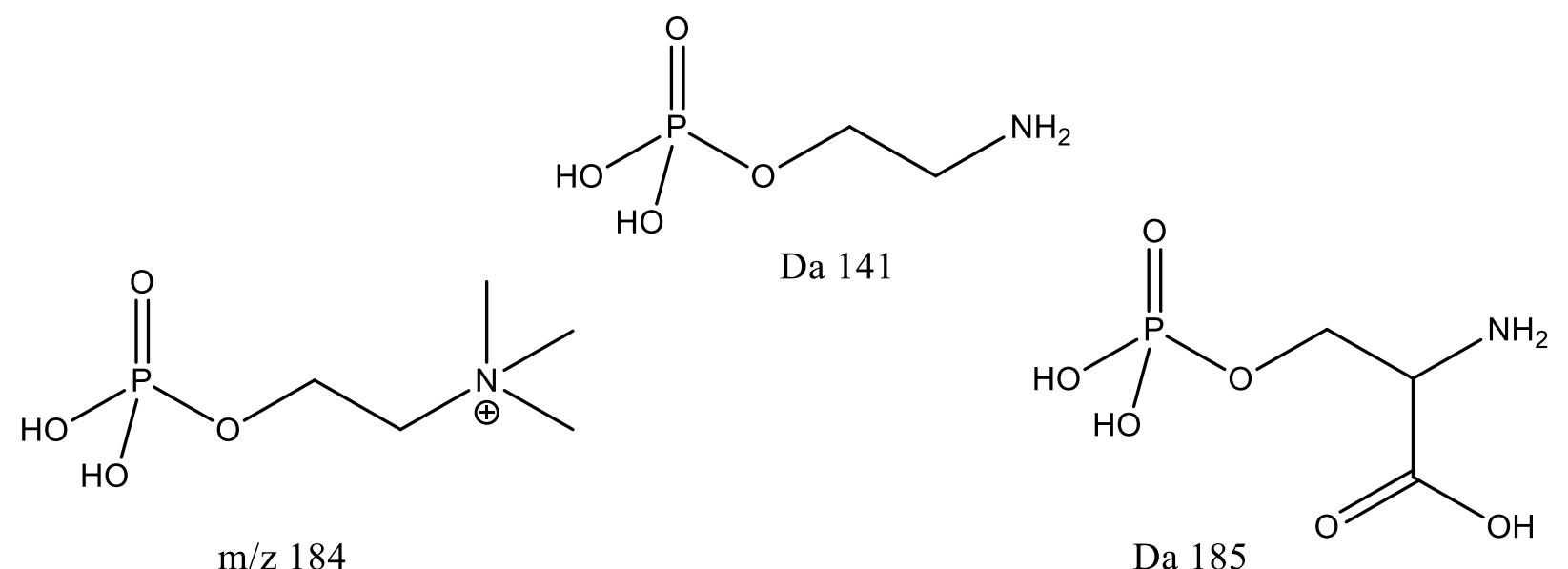

Figure 6:1. $m / z 184$ is the precursor fragment for PC and $S M$; 141 Da is the neutral loss fragment for $P E$; $185 \mathrm{Da}$ is the neutral loss fragment for PS

The four different data sets were collected and are shown for each class of phospholipids in Figure 6:2 for PCs and in Figure 6:3 for SMs (m/z 184 precursor ion scan), PE (141 Da neutral loss scan) and PS (185 Da neutral loss scan). These individual 
chromatograms of a specific phospholipid class are extracted from the total TIC versus time chromatogram between about 24-25 minutes. The Analyst software which collects low-resolution data also allows one to isolate a list of peaks for any time range from TIC chromatograms as text files. One example for this is shown in Figure 6:4, where all the peaks that occur between 24-25 minutes for PC species are isolated into a text file (the list is quite extensive for this time range). The small peak in Figure 6:2 at just about 15 minutes is internal standard peak $\mathrm{PC}(13: 0 / 0: 0)$, and the peak that ranges from 24-30 minutes are all the phospholipids found in goldfish eye retina sample.

The data was collected from TIC chromatograms in 5-minute ranges near baseline intensities and in 1 or 2-minute ranges where high-intensity peaks occurred for the whole run (almost 50 minutes). A total of 15 text files were created for each phospholipid class in every TIC generated. These text files for specific phospholipid class were combined in Excel and masses were grouped by nominal masses in the following rages: $\mathrm{m} / \mathrm{z} 400 \mathrm{~s}$ were separated from .3 to .2 (e.g., 454.3 - 455.2 had nominal mass of 454 ), m/z 500 s were separated from .4 to $.3, \mathrm{~m} / \mathrm{z} 600 \mathrm{~s}$ and $700 \mathrm{~s}$ were separated from .5 to $.4, \mathrm{~m} / \mathrm{z} 800 \mathrm{~s}$ and 900 s were separated from .6 to .5 . This kind of ranges were determined through experience from multiple phospholipid data analyses and seemed to be the most optimal choice for masses separation at low resolution. The peak list for every phospholipid class was generated for all the samples under study and peaks were quantified using the MultiQuant software. 


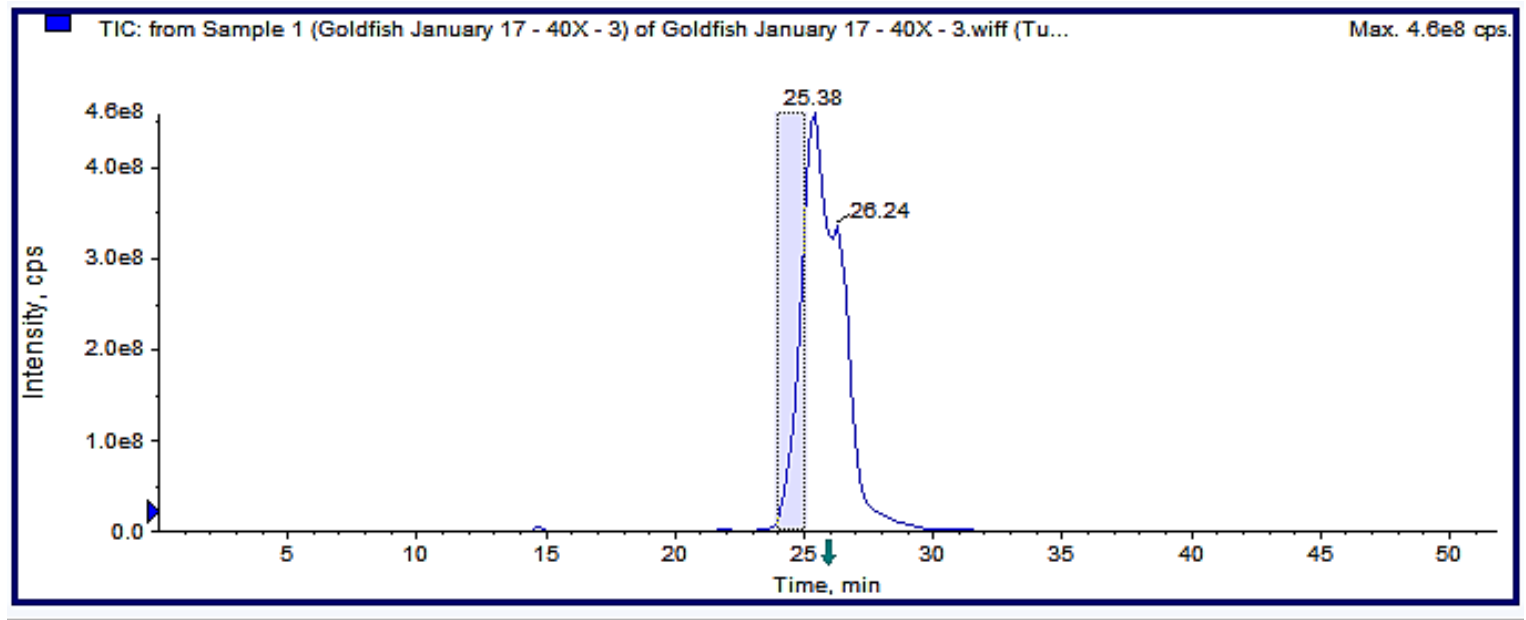

$\square$ +Prec (184.10): Exp 1, 23.930 to 25.101 min from Sample 1 (Goldfish January 17 - 40X - 3... Max. 9.5e5 cps.

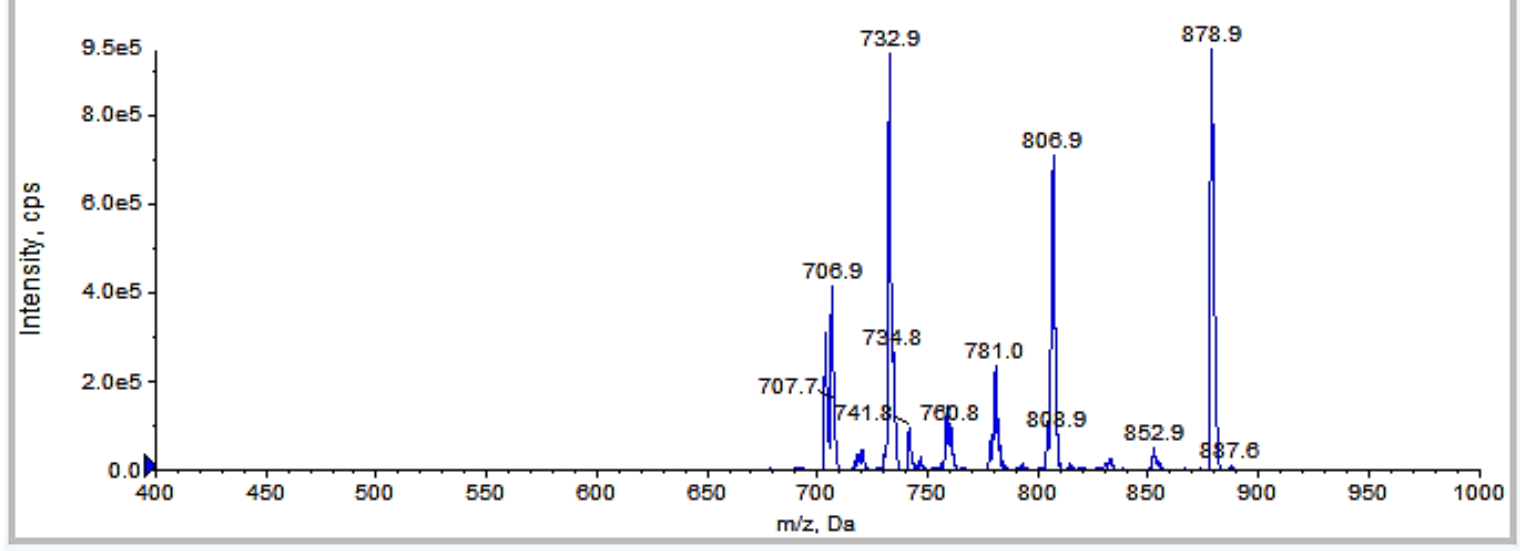

Figure 6:2. The TIC versus time chromatogram is shown in above chromatogram. The below chromatogram shows PC peaks extracted between 24 to 25 minutes from TIC versus time. 

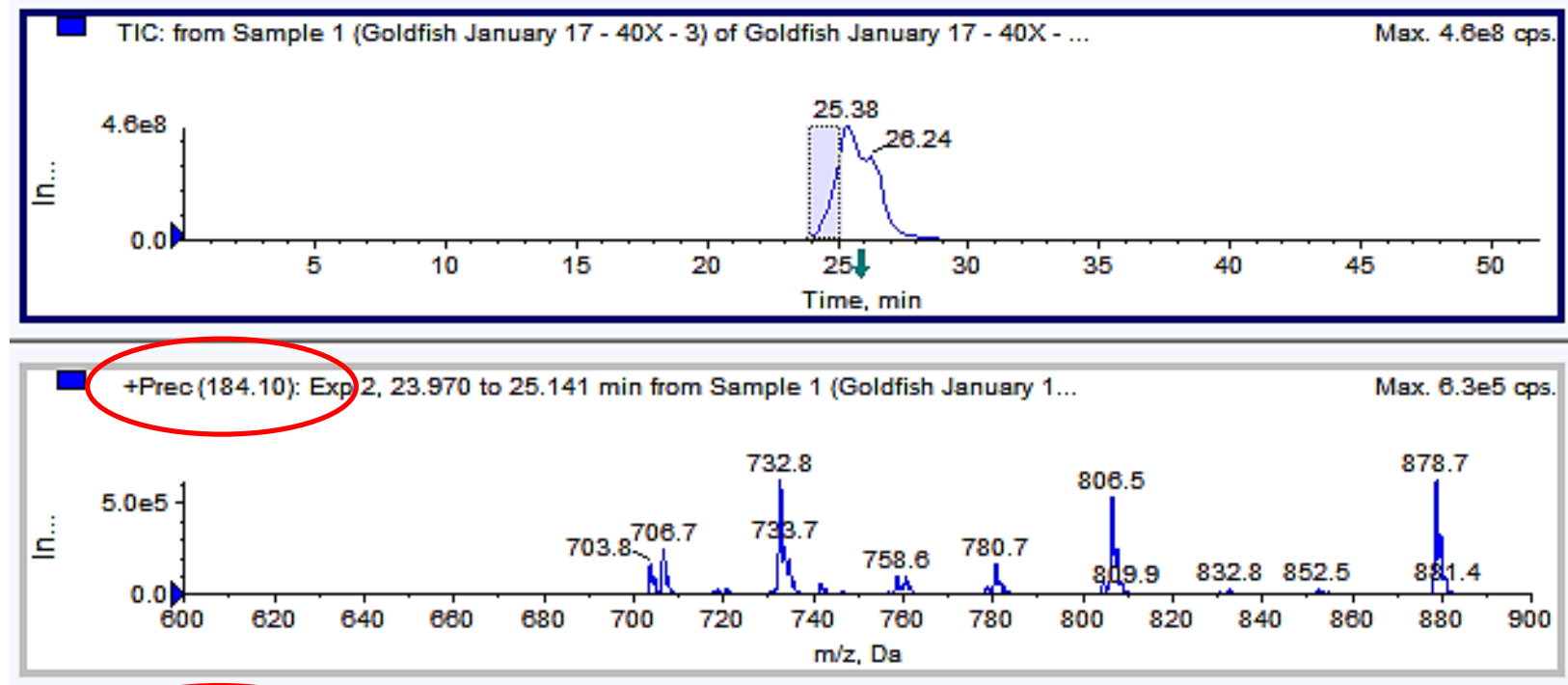

+NL (141.10): Exp 3. 23.990 to 25.162 min from Sample 1 (Goldfish January 17 -..

Max. 5.3e5 cps.

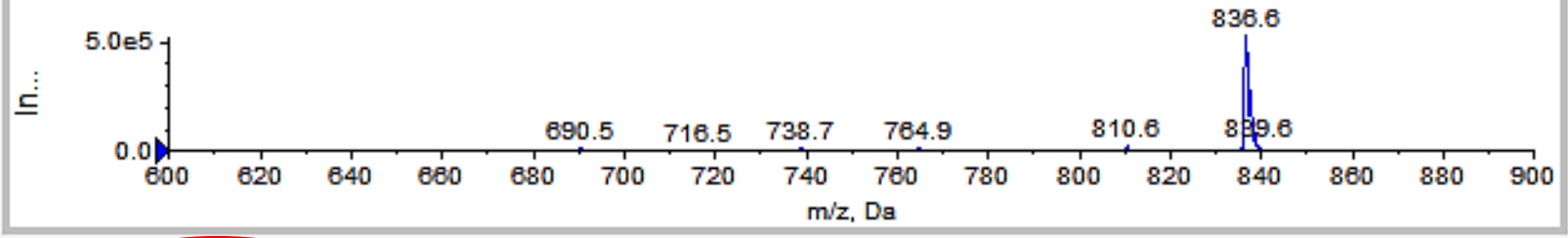

+NL (185.10): Exp 4) 24.030 to 25.202 min from Sample 1 (Goldfish January 17 -...

Max. $5.4 \mathrm{e} 4 \mathrm{cps}$

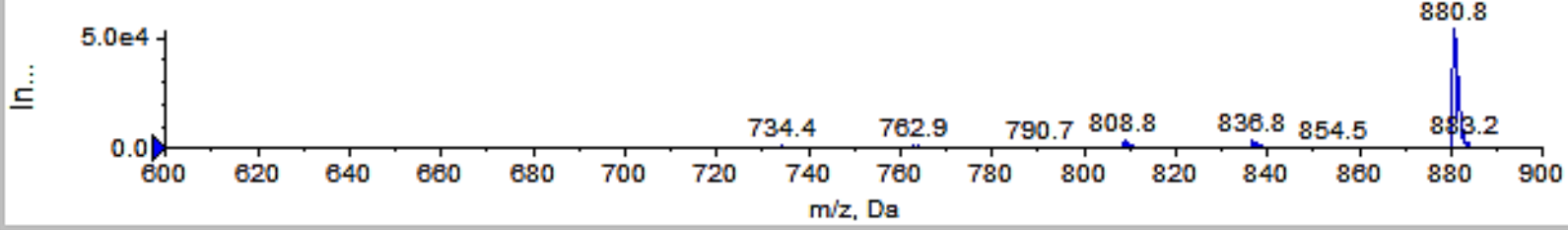

Figure 6:3. The TIC versus time chromatogram is shown in above chromatogram. The below chromatograms show SM ( $\mathrm{m} / z 184$ precursor ion scan), PE (141 Da neutral loss scan) and PS (185 Da neutral loss scan) peaks extracted between 24 to 25 minutes from TIC versus time. 


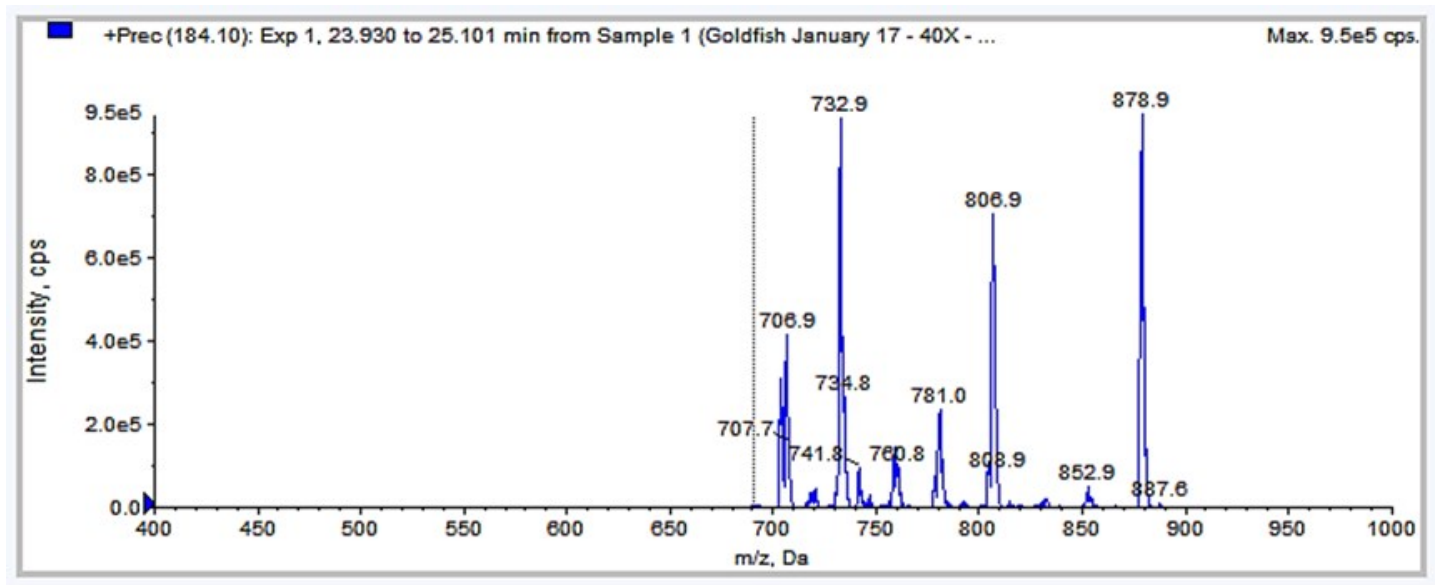

\begin{tabular}{|c|c|c|c|c|c|c|c|}
\hline & $\mathrm{m} / \mathrm{z}(\mathrm{Da})$ & Centroid mass (Da) & Resolution & Intensity (cps) & Peak area (counts) & Charges & $\operatorname{Pe} \wedge$ \\
\hline 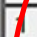 & 703.1000 & $7 0 \longdiv { 3 . 0 9 9 4 }$ & Undefind d & $2.1100 \mathrm{e} 5$ & $8.9932 \mathrm{e} 5$ & Undefined & $\overline{702}$ \\
\hline 2 & 703.9000 & 703.7796 & Undefined & $3.1071 \mathrm{e} 5$ & $2.9086 \mathrm{e} 6$ & 1 & 703 \\
\hline 3 & 704.8000 & 704.8011 & Undefired & $1.2566 \mathrm{e} 5$ & $5.7798 \mathrm{e} 5$ & 1 & 704 \\
\hline 4 & 706.9000 & 706.6735 & 463.9134 & $4.1637 e 5$ & $6.0630 \mathrm{e} 6$ & 1 & 705 \\
\hline 5 & 707.7000 & 707.7000 & Undefired & $1.5536 \mathrm{e} 5$ & $1.0025 e 6$ & 1 & 707 \\
\hline 6 & 707.7000 & 707.9167 & Undefined & $1.5536 \mathrm{e} 5$ & $1.0025 \mathrm{e} 6$ & Undefined & 707 \\
\hline 7 & 709.0000 & $70 \beta .9716$ & Undefined & $2.5632 \mathrm{e} 4$ & $8.3774 \mathrm{e} 4$ & Undefined & 708 \\
\hline 9 & 709.2000 & $7 \longdiv { 9 . 2 0 0 0 }$ & Undefine & $2.0006 \mathrm{e} 4$ & $3.5010 \mathrm{e} 4$ & Undefined & 709 \\
\hline 9 & 716.3000 & 16.3237 & Undefined & 9690.2682 & $5.6579 \mathrm{e} 4$ & Undefined & 715 \\
\hline & $715 \mathrm{n} n \mathrm{n}$ & $717 \cap A 62$ & IIndnfinand & 1 กว01 1 & 117วगn5 & IInAnfinnd & $\begin{array}{c}712 \\
>\end{array}$ \\
\hline
\end{tabular}

Figure 6:4. The peak list that is extracted for PCs from intensity versus $m / z$ chromatogram between 24 to 25 minutes. This peak list data can be extracted as a text file.

Quantification: MultiQuant 2.1.1 software uses .wiff files generated by Analyst and creates extracted ion chromatograms (EIC or XIC) which allow one to plot a single mass (or range of masses) signal versus time. In XIC chromatogram of one or more $\mathrm{m} / \mathrm{z}$ values representing one or more analytes of interest are extracted from the entire data set for a chromatographic run ${ }^{1}$. Once the data is in MultiQuant, peaks are automatically integrated and area values can be extracted, this is shown in Figure $6: 5$ for PE of $\mathrm{m} / \mathrm{z} 820$. For peaks 


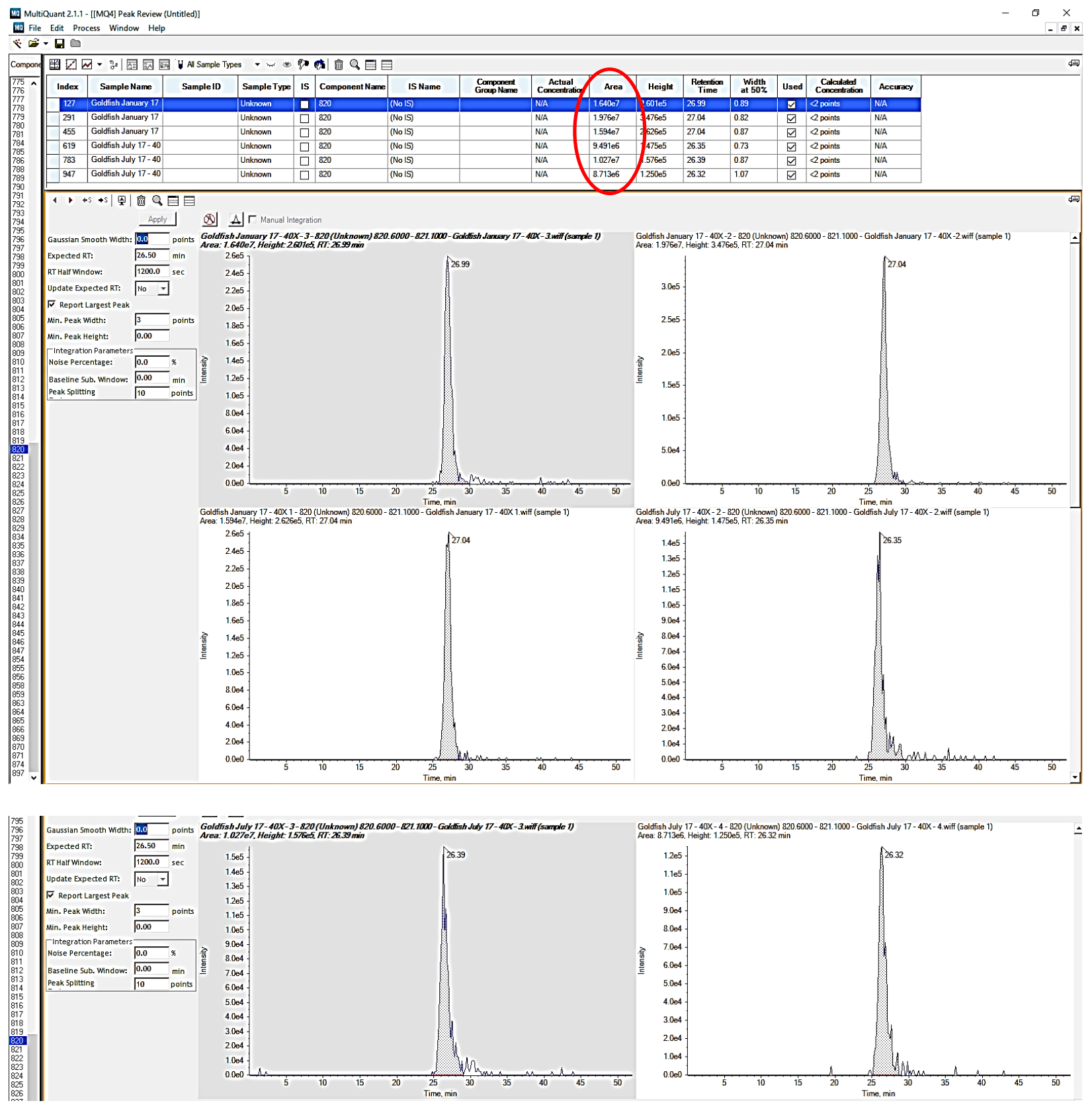

Figure 6:5. MultiQuant 2.1.1. software view. Shown here are area extractions for 820 PE peak for January and July goldfish retina sample in triplicates.

that are not well resolved, manual peak integration is necessary. Manual intergradation is common when peak area values are in the range of limit of quantification (LOQ) values. The LOQ and limit of detection (LOD) values for the AB Sciex QTRAP 4000 have been 
calculated by protocols established by Armbruster and Pry, $2008^{2}$. The whole procedure for LOD and LOQ could be found in a paper by Canez et al., $2016^{3}$ and the values are summarized in Table 6:1. All the peak area values that are below the LOQ are not extracted since it is difficult to quantify them. As long as only one of the three trials is below of $L O Q$, the peak is still extracted for quantification purposes.

Table 6:1. LOD and LOQ values calculated by Canez et al., 2016 by measuring 90 limit of blank along with 90 low concertation standard deviations as described in Armbruster and Pry, 2008. PIS stands for precursor ion scan, and NLS stands for neutral loss scan.

\begin{tabular}{cccc}
\hline Phospholipid class & Scan type & LOD area threshold & LOQ area threshold \\
\hline PC \& SM & PIS 184.1 & $5.02 \times 10^{5}$ & $1.67 \times 10^{6}$ \\
\hline PE & NLS 141.1 & $4.05 \times 10^{5}$ & $1.35 \times 10^{6}$ \\
\hline PS & NLS 185.1 & $1.43 \times 10^{5}$ & $4.77 \times 10^{5}$ \\
\hline
\end{tabular}

Data standardization: The data standardization is done with two ways: first, January and July samples of goldfish eye retina are standardized against each other to eliminate mass variability of the samples (in case of frog, control and dehydrated samples are normalized against each other), second, all the data is standardized against the internal standard. The first step of data standardization was simple with frog samples; as both liver and leg muscle samples were weighted before lipid extraction, so the sample masses can be used in the standardization calculations. The goldfish retina samples were supplied in PBS solution (1 adult goldfish retina in $400 \mu \mathrm{L}$ of PBS) and weighting them was not an option. The first step of standardization in goldfish retina samples was done through the normalizing total sum of all the quantifiable phospholipids found in January with a total sum of all the quantifiable phospholipids found in July. Before applying this standardization step to goldfish retina samples, the standardization method was tested on wood frog liver and leg muscle samples, where mass standardization method was 
compared to a total percentage (\%) of phospholipids standardization method. The ratio values of total \% of phospholipids and masses for dehydrated versus control tissues (July versus January tissues) were calculated and summarized in Table 6:2. The ratio values for wood frog liver between total $\%$ of phospholipids and masses differ by $2.3 \%$, and the same ratio values for wood frog leg muscle differ by $6.8 \%$. The variability between total $\%$ of phospholipids and masses ratios is not very large. However, it would be preferred to standardize with mass data as it is less manipulated data. In the event where there is no information on the weights (in the case of the retina tissue), standardization with total $\%$ of phospholipids is considered reasonable.

Table 6:2. The ratio values of total percentage phospholipids and of masses of dehydrated versus control tissues of wood frog liver and leg muscle tissues and July versus January tissues of goldfish retina tissue.

\begin{tabular}{|c|c|c|c|}
\hline & Control & Dehyd. & $\begin{array}{c}\text { RATIO } \\
\text { (dehyd./control) }\end{array}$ \\
\hline $\begin{array}{c}\text { Total phospholipids in } \\
\text { liver (\%) }\end{array}$ & 98.22 & 99.14 & 1.01 \\
\hline $\begin{array}{c}\text { Internal standard in } \\
\text { liver (\%) }\end{array}$ & 1.78 & 0.86 & Not of interest \\
\hline Mass of liver (g) & 0.1008 & 0.0994 & 0.99 \\
\hline $\begin{array}{c}\text { Total phospholipids in } \\
\text { leg muscle (\%) }\end{array}$ & 90.37 & 91.51 & 1.01 \\
\hline $\begin{array}{l}\text { Internal standard in } \\
\text { leg muscle (\%) }\end{array}$ & 9.63 & 8.49 & Not of interest \\
\hline \multirow[t]{2}{*}{ Mass of leg muscle (g) } & 0.1280 & 0.1390 & 1.08 \\
\hline & January & July & $\begin{array}{c}\text { RATIO } \\
\text { (July/January) }\end{array}$ \\
\hline $\begin{array}{c}\text { Total phospholipids in } \\
\text { retina (\%) }\end{array}$ & 99.61 & 99.58 & 1.00 \\
\hline $\begin{array}{c}\text { Internal standard in } \\
\text { retina (\%) }\end{array}$ & 0.39 & 0.42 & Not of interest \\
\hline
\end{tabular}

Also, all the phospholipids were standardized against the internal standard. All of the phospholipid area values were divided by the internal standard area values, for 
retention time standardization, the retention times of the internal standard were subtracted from all of the phospholipid retention times.

M+2: Carbon, hydrogen, nitrogen, oxygen, and phosphorus are the building blocks of phospholipids, and thus their masses are determined by these atoms. Molecules in nature do not occur as isotopically pure species. Almost all atoms have heavier isotopes that occur in characteristic natural abundances. Carbon occurs typically as ${ }^{12} \mathrm{C}$, but about $1.08 \%$ of carbon atoms are the heavier ${ }^{13} \mathrm{C}$. Hydrogen mostly occurs as ${ }^{1} \mathrm{H}$, but about $0.02 \%$ of hydrogen atoms are ${ }^{2} \mathrm{H}$. Nitrogen occurs predominantly as ${ }^{14} \mathrm{~N}$, but about $0.38 \%$ of it appears as ${ }^{15} \mathrm{~N}$. Oxygen is dominantly ${ }^{16} \mathrm{O}$, but about $0.04 \%$ of oxygen is ${ }^{17} \mathrm{O}$, and $0.20 \%$ of oxygen is ${ }^{18} \mathrm{O}$. Phosphorus occurs as ${ }^{31} \mathrm{P}$ in nature $100 \%$. Peaks caused by ions bearing those heavier isotopes can be found on MS spectra. The relative abundances of such peaks are proportional to the abundances of the isotopes in nature. Most often, the isotopes occur one $(M+1)$ or two $(M+2)$ mass units above the mass of the monoisotopic species. The intensity values of $M+1$ and $M+2$ peaks can be readily found and calculated with online tools, and the calculation of these values is not going to be shown here. However, it is of great importance to consider $M+1$ and $M+2$ peaks when trying to identify phospholipid species, making sure that the monoisotopic peaks are being examined rather than the $M+1$ or $M+2$ peak from another species. Figure 6:6 shows an example of the isotopic distribution of lipid standard $(\mathrm{PC}(13: 0 / 0: 0))$ used in the phospholipid experiments, it could be observed that $\mathrm{M}+1$ and $\mathrm{M}+2$ values are not small and could be easily confused with another phospholipid species. Also, the larger phospholipid nominal mass, the higher isotopic $\mathrm{M}+1$ and $\mathrm{M}+2$ peak abundances it has. 


\section{Isotopic Distribution for $\mathrm{C}_{21} \mathrm{H}_{45} \mathrm{NO}_{7} \mathrm{P}$}

\begin{tabular}{|c|c|c|c|}
\hline Total & $\mathrm{m} / \mathrm{z}$ & $\%$ of Max & Correction Factor \\
\hline M: & 454.2934 & 100.0000 & 1.2936 \\
\hline$M+1:$ & 455.29 & & 5.2448 \\
\hline$M+2=$ & 456.30 & 4.1566 & 31.1209 \\
\hline$M+3$ & 457.3034 & 0.5189 & - \\
\hline$M+4:$ & 458.3068 & 0.0179 & - \\
\hline
\end{tabular}

Isotopic Distribution for $\mathrm{C} 21 \mathrm{H} 45 \mathrm{~N} 07 \mathrm{P}$

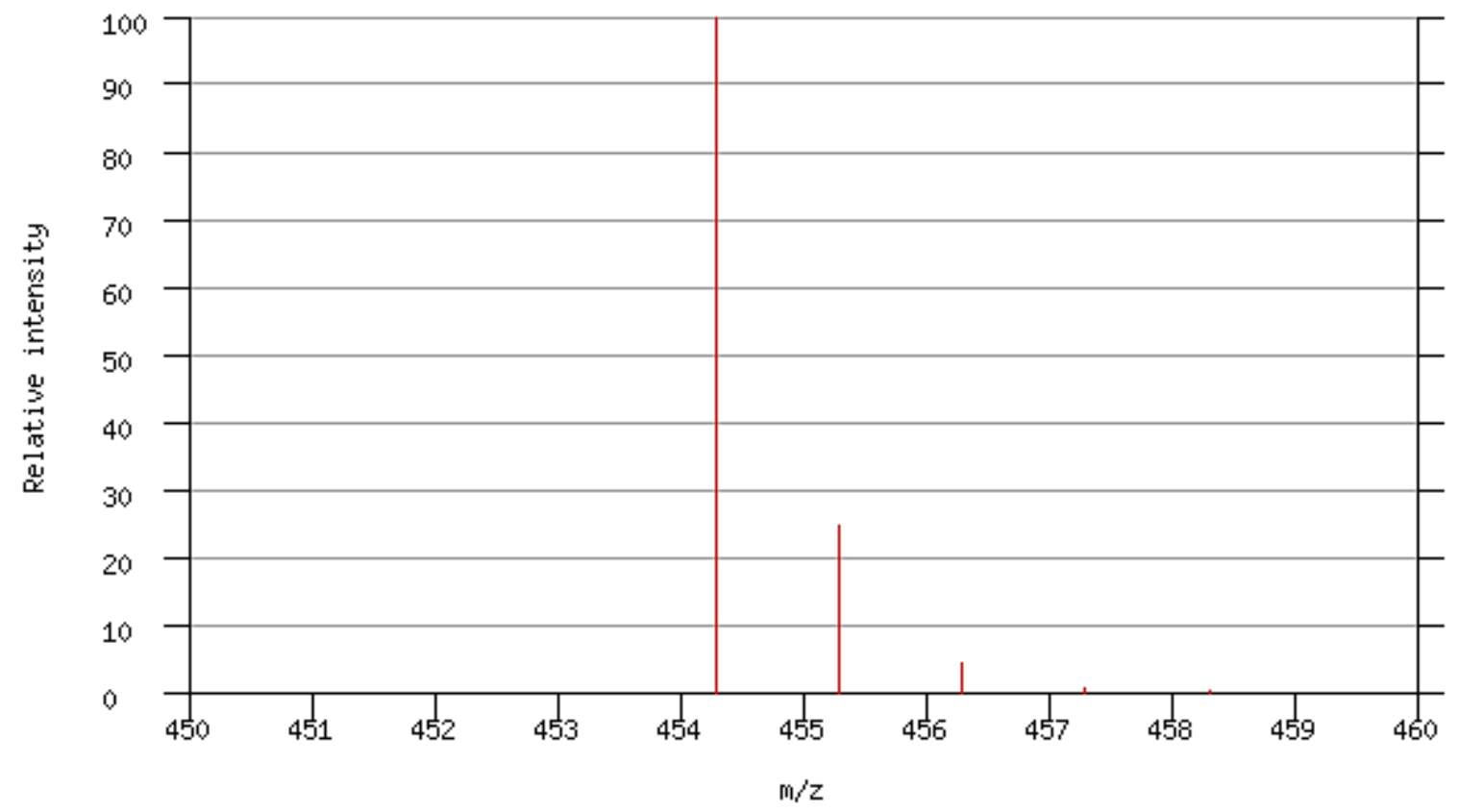

Figure 6:6. Isotopic distribution of $P C(13: 0 / 0: 0)$ lipid. The Figure was generated via the Lipidomics Gateway lipid database.

The next step in data analysis was looking into the data and eliminating all of the peaks that are showing up on the spectra due to them being $M+2$ isotopes of the 
phospholipid species. This was done for all the phospholipid classes studied. In positive ion mode PC, PE and PS species have even nominal masses, and SM species have odd nominal masses ${ }^{4}$. MS analysis of the phospholipids extracted from retina tissue membranes yielded a very complex spectrum; as observed in Figures 6:2 and 6:3 most peaks appear between $\mathrm{m} / \mathrm{z} 700$ to 900 . After the extraction of whole peak lists, it was observed that especially in the range of $\mathrm{m} / \mathrm{z} 700$ to 900 , there is a peak at almost every single unit. LOQ downsized this list by a substantial amount; for goldfish retina membrane, PE chromatograms initially identified 86 candidates however only 48 candidates were above the LOQ.

Figure 6:7 shows $M+2$ calculations for goldfish retina membrane PE species in January done in Excel. It was of interest to figure out the percentage of $M+2$ species to determine if the spectral peaks are more likely $M+2$ species. For example, in Figure 6:7 for $\mathrm{m} / \mathrm{z} 690$ (trial 1$)$, the calculation was $\left[(5.20 \mathrm{E}+06) /(2.39 \mathrm{E}+06)^{*} 100=217.95\right]$. The total column in Figure 6:7 represents the average value of three trials. PE species with the lowest $(\mathrm{m} / \mathrm{z} 674)$ and the highest $(\mathrm{m} / \mathrm{z}$ 866) $M+2$ percentages were looked up from lipid database (Lipidomics Gateway); these values change depending on the list obtained from data extraction. The additional $20 \%$ was added to $M+2$ percentages to ensure not to miss any $M+2$ species due to $L O Q$ and standard deviation of the trials. All the values in Figure 6:7 were sorted in the following way: anything below $29.8 \%$ (marked in dark orange) was determined to be $M+2$ instead of a monoisotopic PE species, anything between $29.8 \%$ 


\begin{tabular}{|c|c|c|c|c|c|c|c|c|c|c|c|}
\hline 4 & A & B & $\mathrm{C}$ & D & $E$ & $\mathrm{~F}$ & G & $\mathrm{H}$ & 1 & J & K \\
\hline 1 & $M / Z$ & \multicolumn{3}{|c|}{ January Areas } & lowest PE & $M+2$ & 9.8 & $M+2$ & highest PE & 17.1 & \\
\hline 2 & & & & & 674 & $M+2+20 \%$ & 29.8 & $M+2+20 \%$ & 866 & 37.1 & \\
\hline 3 & & Trial 1 & Trial 2 & Trial 3 & & & & & & & \\
\hline 4 & & & & & & Trial 1 & Trial 2 & Trial 3 & Total & & \\
\hline 5 & 674 & $1.21 \mathrm{E}+06$ & $1.00 \mathrm{E}+06$ & $1.58 \mathrm{E}+06$ & & & & & & & \\
\hline 6 & 688 & $2.39 \mathrm{E}+06$ & $3.24 \mathrm{E}+06$ & $3.24 \mathrm{E}+06$ & & & & & & & \\
\hline 7 & 690 & $5.20 \mathrm{E}+06$ & $6.30 \mathrm{E}+06$ & $6.28 \mathrm{E}+06$ & & 217.95 & 194.60 & 193.79 & 202.11 & & \\
\hline 8 & 702 & $8.41 E+06$ & $8.01 E+06$ & $7.89 \mathrm{E}+06$ & & & & & & & \\
\hline 9 & 704 & $3.97 \mathrm{E}+06$ & $4.72 E+06$ & $4.60 \mathrm{E}+06$ & & 47.16 & 58.94 & 58.28 & 54.79 & & \\
\hline 10 & 716 & $9.39 \mathrm{E}+06$ & $8.59 \mathrm{E}+06$ & $1.01 \mathrm{E}+07$ & & & & & & & \\
\hline 11 & 718 & $2.08 \mathrm{E}+07$ & $2.25 \mathrm{E}+07$ & $2.16 \mathrm{E}+07$ & & 221.17 & 261.99 & 214.47 & 232.54 & & \\
\hline 12 & 720 & $1.86 \mathrm{E}+06$ & $1.61 \mathrm{E}+06$ & $2.03 E+06$ & & 8.95 & 7.14 & 9.40 & 8.50 & $m+2$ & \\
\hline 13 & 728 & $2.31 E+06$ & $2.44 \mathrm{E}+06$ & $2.69 \mathrm{E}+06$ & & & & & & & \\
\hline 14 & 730 & $6.96 \mathrm{E}+06$ & $8.89 \mathrm{E}+06$ & $7.28 \mathrm{E}+06$ & & 301.08 & 364.90 & 271.01 & 312.33 & & \\
\hline 15 & 732 & $1.41 E+06$ & $1.73 E+06$ & 1.19E +06 & & 20.26 & 19.50 & 16.30 & 18.69 & $m+2$ & \\
\hline 16 & 736 & $1.23 E+06$ & $1.56 \mathrm{E}+06$ & $1.51 \mathrm{E}+06$ & & & & & & & \\
\hline 17 & 738 & $7.16 \mathrm{E}+06$ & $8.34 \mathrm{E}+06$ & $8.19 \mathrm{E}+06$ & & 581.79 & 535.45 & 543.20 & 553.48 & & \\
\hline 18 & 740 & $4.24 \mathrm{E}+06$ & $5.60 \mathrm{E}+06$ & $5.42 \mathrm{E}+06$ & & 59.31 & 67.17 & 66.26 & (1) 64.25 & & \\
\hline 19 & 744 & $1.37 \mathrm{E}+07$ & $1.35 \mathrm{E}+07$ & $1.36 \mathrm{E}+07$ & & & & & & & \\
\hline 20 & 746 & $1.87 \mathrm{E}+07$ & $2.23 \mathrm{E}+07$ & $1.85 \mathrm{E}+07$ & & 136.97 & 165.58 & 136.45 & 146.33 & & \\
\hline 21 & 748 & $5.12 \mathrm{E}+06$ & $5.10 \mathrm{E}+06$ & $5.50 \mathrm{E}+06$ & & 27.38 & 22.84 & 29.68 & ( 26.63 & $m+2$ & \\
\hline 22 & 750 & $1.15 \mathrm{E}+07$ & $1.19 \mathrm{E}+07$ & $1.11 \mathrm{E}+07$ & & 224.52 & 233.47 & 202.22 & 220.07 & & \\
\hline 23 & 752 & $2.84 \mathrm{E}+06$ & $3.44 \mathrm{E}+06$ & $3.39 \mathrm{E}+06$ & & 24.67 & 28.91 & 30.49 & (1) 28.02 & $m+2$ & \\
\hline 24 & 762 & $3.29 \mathrm{E}+06$ & $3.84 \mathrm{E}+06$ & $4.09 \mathrm{E}+06$ & & & & & & & \\
\hline 25 & 764 & $6.89 \mathrm{E}+07$ & $7.01 \mathrm{E}+07$ & $6.94 \mathrm{E}+07$ & & 2094.25 & 1824.05 & 1695.09 & 1871.13 & & \\
\hline 26 & 766 & $3.21 \mathrm{E}+07$ & $3.41 \mathrm{E}+07$ & $3.36 \mathrm{E}+07$ & & - 46.59 & $\quad 48.69$ & (1) 48.36 & (1) 47.88 & & \\
\hline 27 & 768 & $2.90 \mathrm{E}+07$ & $3.16 \mathrm{E}+07$ & $2.88 \mathrm{E}+07$ & & 90.37 & 92.64 & 85.93 & 89.65 & & \\
\hline 28 & 770 & $5.50 \mathrm{E}+06$ & $7.43 E+06$ & $6.08 \mathrm{E}+06$ & & 18.96 & 23.51 & 21.08 & 21.18 & $m+2$ & \\
\hline 29 & 772 & $4.14 \mathrm{E}+06$ & $4.04 \mathrm{E}+06$ & $4.80 \mathrm{E}+06$ & & 75.34 & 54.40 & 78.93 & 69.56 & & \\
\hline 30 & 774 & $5.57 \mathrm{E}+06$ & $6.23 \mathrm{E}+06$ & $6.13 E+06$ & & 134.54 & 154.02 & 127.75 & 138.77 & & \\
\hline 31 & 776 & $2.01 \mathrm{E}+07$ & $2.29 \mathrm{E}+07$ & $2.27 \mathrm{E}+07$ & & 359.89 & 367.27 & 370.49 & 365.88 & & \\
\hline 32 & 778 & $1.79 \mathrm{E}+07$ & $2.02 \mathrm{E}+07$ & $2.25 \mathrm{E}+07$ & & 89.38 & 88.28 & 98.90 & ( $\quad 92.19$ & & \\
\hline 33 & 780 & $2.46 \mathrm{E}+06$ & $2.74 \mathrm{E}+06$ & $3.19 \mathrm{E}+06$ & & 13.73 & 13.56 & 14.20 & 13.83 & $m+2$ & \\
\hline 34 & 786 & $1.85 \mathrm{E}+06$ & $1.79 \mathrm{E}+06$ & $1.83 \mathrm{E}+06$ & & & & & & & \\
\hline 35 & 788 & $2.84 \mathrm{E}+06$ & $3.44 \mathrm{E}+06$ & $2.54 \mathrm{E}+06$ & & - 153.10 & 192.29 & 138.73 & 161.37 & & \\
\hline 36 & 790 & $4.68 \mathrm{E}+07$ & $5.33 \mathrm{E}+07$ & $5.06 \mathrm{E}+07$ & & 1650.69 & 1550.29 & 1995.27 & 1732.08 & & \\
\hline 37 & 792 & $2.38 \mathrm{E}+08$ & $2.67 \mathrm{E}+08$ & 2. $60 \mathrm{E}+08$ & & 508.86 & 501.41 & (1) 514.62 & (1) 508.30 & & \\
\hline 38 & 794 & $4.32 \mathrm{E}+07$ & $4.65 \mathrm{E}+07$ & 4. $63 \mathrm{E}+07$ & & 18.15 & 17.40 & 17.77 & ( $\quad 17.77$ & $m+2$ & \\
\hline 39 & 796 & $3.49 \mathrm{E}+06$ & $4.19 \mathrm{E}+06$ & $3.37 \mathrm{E}+06$ & & 8.07 & 9.01 & 7.27 & 8.12 & $m+2$ & \\
\hline 40 & 810 & $6.86 \mathrm{E}+06$ & $8.16 \mathrm{E}+06$ & $7.51 \mathrm{E}+06$ & & & & & & & \\
\hline 41 & 812 & $4.85 \mathrm{E}+06$ & $5.68 \mathrm{E}+06$ & $5.02 \mathrm{E}+06$ & & 70.69 & 69.54 & 66.89 & 69.04 & & \\
\hline 42 & 814 & $2.74 \mathrm{E}+06$ & $3.17 \mathrm{E}+06$ & $2.75 \mathrm{E}+06$ & & 56.52 & 55.79 & 54.76 & 55.69 & & \\
\hline 43 & 814 & $3.37 E+06$ & $3.88 \mathrm{E}+06$ & $4.29 \mathrm{E}+06$ & & & & & & & \\
\hline 44 & 816 & $2.49 \mathrm{E}+06$ & $2.74 \mathrm{E}+06$ & 2. $69 \mathrm{E}+06$ & & 90.76 & 86.45 & 97.71 & 91.64 & & \\
\hline 45 & 818 & $1.33 E+07$ & $1.54 \mathrm{E}+07$ & $1.31 \mathrm{E}+07$ & & 536.20 & 561.56 & 487.90 & 528.56 & & \\
\hline 46 & 820 & $1.59 \mathrm{E}+07$ & $1.98 \mathrm{E}+07$ & 1. $64 \mathrm{E}+07$ & & 119.58 & 128.56 & 125.10 & ( 124.41 & & \\
\hline 47 & 822 & $2.34 \mathrm{E}+06$ & $3.52 \mathrm{E}+06$ & $2.46 \mathrm{E}+06$ & & 14.65 & 17.79 & 15.01 & (15.81 & $m+2$ & \\
\hline 48 & 836 & $4.71 E+08$ & $4.72 E+08$ & $4.81 E+08$ & & & & & & & \\
\hline 49 & 838 & $7.03 E+07$ & $7.76 \mathrm{E}+07$ & $7.21 \mathrm{E}+07$ & & 14.91 & 16.43 & 14.97 & 15.44 & $m+2$ & \\
\hline 50 & 840 & $4.87 \mathrm{E}+06$ & $4.39 \mathrm{E}+06$ & $5.05 \mathrm{E}+06$ & & 6.93 & 5.66 & 7.00 & 6.53 & $m+2$ & \\
\hline 51 & 864 & $5.60 E+06$ & $6.08 \mathrm{E}+06$ & $4.97 E+06$ & & & & & & & \\
\hline 52 & 866 & $2.54 \mathrm{E}+06$ & $2.86 \mathrm{E}+06$ & $2.08 \mathrm{E}+06$ & & 45.29 & 47.11 & 41.91 & 44.77 & & \\
\hline 53 & & & & & & & & & & & \\
\hline
\end{tabular}

Figure 6:7. $M+2$ calculations for goldfish retina membrane PE species in January done in Excel. 


\begin{tabular}{|c|c|c|c|c|c|c|c|c|c|}
\hline L & $\mathrm{M}$ & $\mathrm{N}$ & $\mathrm{O}$ & $\mathrm{P}$ & Q & $\mathrm{R}$ & 5 & $\mathrm{~T}$ & $\mathrm{U}$ \\
\hline \multirow[t]{4}{*}{$M / Z$} & \multicolumn{3}{|c|}{ July Areas } & lowest PE & $M+2$ & 9.8 & $M+2$ & highest PE & 17.1 \\
\hline & & & & 674 & $M+2+20 \%$ & 29.8 & $M+2+20 \%$ & 866 & 37.1 \\
\hline & Trial 1 & Trial 2 & Trial 3 & & & & & & \\
\hline & & & & & Trial 1 & Trial 2 & Trial 3 & Total & \\
\hline 674 & $1.71 \mathrm{E}+06$ & $1.66 \mathrm{E}+06$ & $1.13 E+06$ & & & & & & \\
\hline 688 & $1.36 \mathrm{E}+06$ & $2.13 E+06$ & $1.66 \mathrm{E}+06$ & & & & & & \\
\hline 690 & $3.89 \mathrm{E}+06$ & $4.80 E+06$ & $4.52 \mathrm{E}+06$ & & 287.02 & 224.74 & 272.78 & 261.52 & \\
\hline 702 & $5.83 E+06$ & $4.45 E+06$ & $5.75 \mathrm{E}+06$ & & & & & & \\
\hline 704 & $2.21 \mathrm{E}+06$ & $2.08 \mathrm{E}+06$ & $1.96 \mathrm{E}+06$ & & 37.93 & 46.88 & 34.07 & 39.63 & \\
\hline 716 & $5.60 \mathrm{E}+06$ & $7.16 \mathrm{E}+06$ & $7.83 E+06$ & & & & & & \\
\hline 718 & $1.58 \mathrm{E}+07$ & $1.55 \mathrm{E}+07$ & $1.87 \mathrm{E}+07$ & & 282.55 & 217.16 & 239.09 & 246.27 & \\
\hline 720 & $1.43 E+06$ & $1.98 \mathrm{E}+06$ & $1.78 \mathrm{E}+06$ & & 9.05 & 12.77 & 9.52 & 10.44 & $m+2$ \\
\hline 728 & $3.14 \mathrm{E}+06$ & $2.96 \mathrm{E}+06$ & $3.26 \mathrm{E}+06$ & & & & & & \\
\hline 730 & $4.34 \mathrm{E}+06$ & $4.17 E+06$ & $3.97 E+06$ & & 138.39 & 140.67 & 121.54 & 133.53 & \\
\hline 732 & $7.28 \mathrm{E}+05$ & $6.28 \mathrm{E}+05$ & $6.88 \mathrm{E}+05$ & & 16.76 & 15.06 & 17.35 & 16.39 & $m+2$ \\
\hline 736 & $1.26 \mathrm{E}+06$ & $1.26 \mathrm{E}+06$ & $1.46 \mathrm{E}+06$ & & & & & & \\
\hline 738 & $2.69 \mathrm{E}+06$ & $3.92 \mathrm{E}+06$ & $2.74 \mathrm{E}+06$ & & 214.10 & 312.11 & 187.98 & 238.07 & \\
\hline 740 & $4.80 \mathrm{E}+06$ & $5.10 \mathrm{E}+06$ & $5.27 \mathrm{E}+06$ & & 178.49 & 130.13 & 192.66 & 167.09 & \\
\hline 744 & $9.22 \mathrm{E}+06$ & $1.08 \mathrm{E}+07$ & $9.79 \mathrm{E}+06$ & & & & & & \\
\hline 746 & $1.85 \mathrm{E}+07$ & $1.82 E+07$ & 1. $63 \mathrm{E}+07$ & & 201.09 & 168.52 & 166.65 & 178.75 & \\
\hline 748 & $4.60 \mathrm{E}+06$ & $4.67 E+06$ & $4.67 \mathrm{E}+06$ & & 24.80 & 25.66 & 28.62 & 26.36 & $m+2$ \\
\hline 750 & $1.27 \mathrm{E}+07$ & $1.26 \mathrm{E}+07$ & $1.35 \mathrm{E}+07$ & & 275.52 & 269.32 & 289.29 & 278.04 & \\
\hline 752 & $3.42 \mathrm{E}+06$ & $3.19 E+06$ & $2.94 \mathrm{E}+06$ & & 26.97 & 25.35 & 21.75 & 24.69 & $m+2$ \\
\hline 762 & $2.26 \mathrm{E}+06$ & $3.37 E+06$ & $3.04 \mathrm{E}+06$ & & & & & & \\
\hline 764 & $6.22 \mathrm{E}+07$ & $7.02 E+07$ & $6.65 \mathrm{E}+07$ & & 2750.88 & 2086.48 & 2188.61 & 2341.99 & \\
\hline 766 & $1.84 \mathrm{E}+07$ & $2.31 \mathrm{E}+07$ & $2.03 E+07$ & & 29.61 & 32.87 & 30.59 & (1) $\quad 31.03$ & \\
\hline 768 & $2.73 E+07$ & $3.10 \mathrm{E}+07$ & $2.85 \mathrm{E}+07$ & & 148.40 & 134.27 & 139.87 & 140.85 & \\
\hline 770 & $5.93 E+06$ & $6.00 E+06$ & $5.60 \mathrm{E}+06$ & & 21.69 & 19.36 & 19.68 & 20.25 & $m+2$ \\
\hline 772 & $2.69 \mathrm{E}+06$ & $3.52 E+06$ & $2.46 \mathrm{E}+06$ & & 45.34 & 58.57 & 43.95 & 49.29 & \\
\hline 774 & $5.30 \mathrm{E}+06$ & $6.43 E+06$ & $5.32 \mathrm{E}+06$ & & 197.17 & 182.87 & 216.29 & 198.78 & \\
\hline 776 & $2.10 \mathrm{E}+07$ & $2.46 \mathrm{E}+07$ & $2.36 \mathrm{E}+07$ & & 396.75 & 382.86 & 443.92 & 407.84 & \\
\hline 778 & $2.11 E+07$ & $2.28 \mathrm{E}+07$ & $2.09 \mathrm{E}+07$ & & 100.33 & 92.65 & 88.40 & 93.79 & \\
\hline 780 & $2.03 E+06$ & $2.51 E+06$ & $2.39 \mathrm{E}+06$ & & 9.64 & 11.01 & 11.42 & 10.69 & $m+2$ \\
\hline 786 & $9.69 \mathrm{E}+05$ & $1.06 \mathrm{E}+06$ & $1.16 \mathrm{E}+06$ & & & & & & \\
\hline 788 & $3.14 \mathrm{E}+06$ & $3.97 E+06$ & $3.79 \mathrm{E}+06$ & & 324.08 & 374.60 & 328.03 & (1) 342.23 & \\
\hline 790 & $4.52 \mathrm{E}+07$ & $5.08 \mathrm{E}+07$ & $4.67 \mathrm{E}+07$ & & ( 1438.99 & 1279.81 & 1232.33 & 1317.04 & \\
\hline 792 & $2.44 \mathrm{E}+08$ & $2.76 \mathrm{E}+08$ & $2.59 \mathrm{E}+08$ & & ( $\quad 540.40$ & 543.83 & (1) 554.03 & 546.09 & \\
\hline 794 & $4.34 \mathrm{E}+07$ & $4.67 \mathrm{E}+07$ & $4.13 E+07$ & & 17.77 & 16.90 & 15.94 & 16.87 & $m+2$ \\
\hline 796 & $2.24 \mathrm{E}+06$ & $3.04 E+06$ & $2.69 \mathrm{E}+06$ & & 5.15 & 6.51 & 6.51 & 6.06 & $m+2$ \\
\hline 810 & $4.24 \mathrm{E}+06$ & $4.67 E+06$ & $4.85 \mathrm{E}+06$ & & & & & & \\
\hline 812 & $4.32 \mathrm{E}+06$ & $5.10 E+06$ & $4.90 \mathrm{E}+06$ & & 101.77 & 109.14 & 101.03 & 103.98 & \\
\hline 814 & $2.52 \mathrm{E}+06$ & $3.13 E+06$ & $2.70 \mathrm{E}+06$ & & 58.42 & 61.33 & 55.07 & 58.27 & \\
\hline 814 & $2.28 \mathrm{E}+06$ & $3.52 E+06$ & $3.09 \mathrm{E}+06$ & & & & & & \\
\hline 816 & $2.44 \mathrm{E}+06$ & $2.39 \mathrm{E}+06$ & $2.44 \mathrm{E}+06$ & & 96.55 & 76.30 & 90.36 & 87.73 & \\
\hline 818 & $1.03 E+07$ & $1.17 \mathrm{E}+07$ & $1.14 \mathrm{E}+07$ & & 422.82 & 490.57 & 465.93 & 459.77 & \\
\hline 820 & $9.49 \mathrm{E}+06$ & $1.03 E+07$ & $8.71 E+06$ & & 92.15 & 87.78 & 76.77 & 85.56 & \\
\hline 822 & $1.58 \mathrm{E}+06$ & $1.61 E+06$ & $2.03 E+06$ & & 16.67 & 15.65 & 23.34 & 18.55 & $m+2$ \\
\hline 836 & $4.33 E+08$ & $4.82 E+08$ & $4.82 \mathrm{E}+08$ & & & & & & \\
\hline 838 & $6.69 \mathrm{E}+07$ & $7.23 \mathrm{E}+07$ & $7.21 \mathrm{E}+07$ & & 15.44 & 15.01 & 14.96 & 15.14 & $m+2$ \\
\hline 840 & $3.47 E+06$ & $3.89 \mathrm{E}+06$ & $4.07 E+06$ & & 5.18 & 5.38 & 5.65 & 5.40 & $m+2$ \\
\hline 864 & $4.70 \mathrm{E}+06$ & $4.55 E+06$ & $4.62 E+06$ & & & & & & \\
\hline 866 & $2.08 \mathrm{E}+06$ & $2.69 \mathrm{E}+06$ & $2.06 \mathrm{E}+06$ & & 44.38 & 59.12 & 44.57 & 49.36 & \\
\hline
\end{tabular}

Figure 6:8. $M+2$ calculations for goldfish retina membrane PE species in July done in Excel. 
and $37.1 \%$ (marked in yellow) was investigated by their unique $\mathrm{M}+2$ percentage values, anything above $37.1 \%$ (marked in green) was determined to be a monoisotopic PE species. In Figure 6:7 it was found that from 48 PE candidates, 11 were $M+2$ peaks of other PE species. Figure 6:8 shows $\mathrm{M}+2$ calculations for goldfish retina membrane $\mathrm{PE}$ species in July. It could be observed that Figures 6:7 and 6:8 show same results. In Figure $6: 8 \mathrm{~m} / \mathrm{z} 766$ is also yellow, so it was looked at separately to see if it is an $M+2$ peak. For $\mathrm{m} / \mathrm{z} 764$, the $\mathrm{M}+2+20 \%$ value is $33.3 \%$ which is smaller than the mean value of peak $\mathrm{m} / \mathrm{z}$ 766 in Tables 6:7 and 6:8 $[(47.88+31.03) \% / 2]=39.4 \%$; for this reason, $\mathrm{m} / \mathrm{z} 766$ peak was not considered as potential $M+2$ peak. The final list of PE species before quantification consisted of 37 PEs.

It could be observed that $\mathrm{m} / \mathrm{z} 814$ has two peaks in Tables $6: 7$ and $6: 8$ because there were two peaks found for that nominal mass above LOQ (two peaks with same nominal mass are written in blue). The $\mathrm{M}+2$ calculations in this kind of situation were carried out for peaks with similar retention times. The m/z peak $814(2.52 \mathrm{E}+06,3.13 \mathrm{E}+06$, 2.70E+06) in Figure 6:8 has a retention time of $25.6 \mathrm{~min}$, the other $\mathrm{m} / \mathrm{z} 814$ peak has a retention time of $26.9 \mathrm{~min}$. The first $\mathrm{m} / \mathrm{z} 814$ peak was used in $M+2$ calculations as its retention time is closer to $\mathrm{m} / \mathrm{z} 812(25.2 \mathrm{~min})$ and $\mathrm{m} / \mathrm{z} 816(26.0)$ than the other $\mathrm{m} / \mathrm{z} 814$ peak.

$\mathrm{M}+1$ : $\mathrm{PC}$ and SM species appear on the same chromatogram as they both have the same choline head group. It was also of interest to eliminate all of the $M+1$ isotopes from the peak lists (PC and SM nominal masses differ only by one unit). $M+1$ calculations were carried out with the same guidelines as the calculations for $M+2$ peaks shown in Figures 
6:7 and 6:8. The whole calculation for $M+1$ peaks for goldfish retina membrane $P C$ and SM species in January is shown in Figure 6:9. The total of $64 \mathrm{PC}$ and SM M+1 peaks were found. The $\mathrm{M}+1$ calculation results for July were quite similar to the ones obtained in Figure 6:9, though not shown here. After eliminating all of the possible $M+1$ and $M+2$ candidates, the PC list consisted of 90 lipids and SM list consisted of 19 lipids. The list for PCs did not come from Figure 6:9 as PCs were collected in the broader range $(\mathrm{m} / \mathrm{z}$ 400-1000) than SMs (m/z 600-900), in Figure 6:9 PCs are just used for $M+1$ peaks calculations.

The mass ranges for the phospholipid analyses were based on previous work in the literature ${ }^{5,6}$, through lipidomic pattern observations in the lipids database as well as analysis of our data. It could be observed from Figures $6: 2$ and 6:3 that most of the peaks for phospholipids were in the range of $\mathrm{m} / \mathrm{z} 700-900$. Also, frequent use of lipid database (Lipidomics Gateway) has shown that there are no phospholipids observed under $400 \mathrm{Da}$ and quite rarely observed over $1000 \mathrm{Da}$, the smallest SM in the lipid database could be found in $600 \mathrm{Da}$ range. The SMs could also be observed in PC spectra, and they were all in the range of $\mathrm{m} / \mathrm{z} 600-900$. PCs (as well as PEs and PSs) were analyzed via lowresolution scans, and SMs were analyzed at the unit resolution on the QTRAP MS instrument; this was done to better distinguish between PCs and SMs as they are only one Da apart in mass. Low-resolution scanning leads to higher sensitivity in MS, while unit resolution improves mass accuracy ${ }^{7}$. 


\begin{tabular}{|c|c|c|c|c|c|c|c|c|c|c|}
\hline$\Delta$ & A & $\mathrm{B}$ & $\mathrm{C}$ & D & $J$ & $\mathrm{~K}$ & $\mathrm{~L}$ & $\mathrm{M}$ & $\mathrm{N}$ & 0 \\
\hline 1 & $\mathrm{M} / \mathrm{Z}$ & \multicolumn{3}{|c|}{ January Areas } & lowest PC & $\mathrm{M}+1$ & 41.8 & $M+1$ & highest PC & 60.06 \\
\hline 2 & & & & & 676 & $M+1+20 \%$ & 61.8 & $M+1+20 \%$ & 898 & 80.06 \\
\hline 3 & & & & & lowest SM & $\mathrm{M}+1$ & 43.25 & $M+1$ & highest SM & 60.9 \\
\hline 4 & & & & & 675 & $M+1+20 \%$ & 63.25 & $M+1+20 \%$ & 899 & 80.9 \\
\hline 5 & & Trial 1 & Trial 2 & Trial 3 & & & & & & \\
\hline 6 & & & & & & $\mathrm{PC}(\mathrm{M}+1)$ & $S M(M+1)$ & & & \\
\hline 7 & 675 & $1.49 \mathrm{E}+07$ & $7.46 \mathrm{E}+06$ & $2.39 E+06$ & & & & & & \\
\hline 8 & 676 & $5.69 \mathrm{E}+06$ & $2.59 E+06$ & $6.28 \mathrm{E}+05$ & & & 33.04 & $M+1$ & & \\
\hline 9 & 678 & $5.60 \mathrm{E}+06$ & $5.88 \mathrm{E}+06$ & $5.57 \mathrm{E}+06$ & & & & & & \\
\hline 10 & 679 & $1.41 \mathrm{E}+06$ & $1.71 \mathrm{E}+06$ & $1.98 \mathrm{E}+06$ & & ○ 29.92 & & $M+1$ & & \\
\hline 11 & 689 & $1.88 \mathrm{E}+06$ & $1.91 \mathrm{E}+06$ & $1.23 E+06$ & & & & & & \\
\hline 12 & 703 & $7.78 \mathrm{E}+07$ & $9.03 E+07$ & $7.44 \mathrm{E}+07$ & & & & & & \\
\hline 13 & 704 & $4.38 \mathrm{E}+07$ & $4.98 \mathrm{E}+07$ & $4.08 \mathrm{E}+07$ & & & 55.43 & $M+1$ & & \\
\hline 14 & 706 & $1.13 E+08$ & $1.25 \mathrm{E}+08$ & $1.08 \mathrm{E}+08$ & & & & & & \\
\hline 15 & 707 & $4.16 \mathrm{E}+07$ & $4.99 E+07$ & $4.19 E+07$ & & 38.57 & & $M+1$ & & \\
\hline 16 & 708 & $9.17 \mathrm{E}+06$ & $1.21 \mathrm{E}+07$ & $7.89 \mathrm{E}+06$ & & & 21.73 & $M+1$ & & \\
\hline 17 & 716 & $6.15 \mathrm{E}+06$ & $5.75 \mathrm{E}+06$ & $5.05 E+06$ & & & & & & \\
\hline 18 & 717 & $5.98 \mathrm{E}+06$ & $6.45 E+06$ & $5.30 \mathrm{E}+06$ & & 104.78 & & & & \\
\hline 19 & 718 & $3.01 E+07$ & $2.92 E+07$ & $2.68 \mathrm{E}+07$ & & & ○ 487.25 & & & \\
\hline 20 & 719 & $1.30 \mathrm{E}+07$ & $1.19 \mathrm{E}+07$ & $1.10 \mathrm{E}+07$ & & 41.61 & & $M+1$ & & \\
\hline 21 & 720 & $1.86 \mathrm{E}+07$ & $1.92 \mathrm{E}+07$ & $1.72 \mathrm{E}+07$ & & & 153.82 & & & \\
\hline 22 & 721 & $6.88 \mathrm{E}+06$ & $7.33 E+06$ & $6.65 E+06$ & & ( 37.95 & & $M+1$ & & \\
\hline 23 & 730 & $1.16 \mathrm{E}+07$ & $1.19 \mathrm{E}+07$ & $1.11 \mathrm{E}+07$ & & & & & & \\
\hline 24 & 731 & $3.74 \mathrm{E}+07$ & $3.35 E+07$ & $2.74 \mathrm{E}+07$ & & 283.02 & & & & \\
\hline 25 & 732 & $3.21 \mathrm{E}+08$ & $3.36 \mathrm{E}+08$ & $2.84 \mathrm{E}+08$ & & & ○ 965.54 & & & \\
\hline 26 & 733 & $1.33 E+08$ & $1.31 \mathrm{E}+08$ & $1.20 \mathrm{E}+08$ & & ( 40.80 & & $M+1$ & & \\
\hline 27 & 734 & $2.81 E+08$ & $2.74 \mathrm{E}+08$ & $2.43 E+08$ & & & 208.19 & & & \\
\hline 28 & 735 & $1.04 \mathrm{E}+08$ & $9.94 \mathrm{E}+07$ & $8.99 E+07$ & & (1) 36.73 & & $M+1$ & & \\
\hline 29 & 736 & $2.16 \mathrm{E}+07$ & $2.20 \mathrm{E}+07$ & $1.99 \mathrm{E}+07$ & & & 21.68 & $M+1$ & & \\
\hline 30 & 741 & $2.88 \mathrm{E}+07$ & $3.26 \mathrm{E}+07$ & $2.63 E+07$ & & & & & & \\
\hline 31 & 742 & $1.77 \mathrm{E}+07$ & $1.67 \mathrm{E}+07$ & $1.50 \mathrm{E}+07$ & & & 56.52 & $M+1$ & & \\
\hline 32 & 744 & $3.01 E+07$ & $2.86 \mathrm{E}+07$ & $2.61 \mathrm{E}+07$ & & & & & & \\
\hline 33 & 745 & $1.32 \mathrm{E}+07$ & 1.33E+07 & $1.19 \mathrm{E}+07$ & & 45.17 & & $M+1$ & & \\
\hline 34 & 746 & $8.93 E+07$ & $9.10 \mathrm{E}+07$ & $8.31 E+07$ & & & 688.92 & & & \\
\hline 35 & 747 & $3.76 \mathrm{E}+07$ & $3.51 E+07$ & $3.22 \mathrm{E}+07$ & & 39.80 & & $M+1$ & & \\
\hline 36 & 748 & $1.21 \mathrm{E}+07$ & $1.40 \mathrm{E}+07$ & $1.27 \mathrm{E}+07$ & & & 37.07 & $M+1$ & & \\
\hline 37 & 756 & $6.96 \mathrm{E}+06$ & $6.96 E+06$ & $5.83 E+06$ & & & & & & \\
\hline 38 & 757 & $3.19 E+06$ & $3.01 \mathrm{E}+06$ & $2.51 E+06$ & & 44.09 & & $M+1$ & & \\
\hline 39 & 758 & $7.92 \mathrm{E}+07$ & $8.09 E+07$ & $7.29 \mathrm{E}+07$ & & & 2690.66 & & & \\
\hline 40 & 759 & $5.05 E+07$ & $4.91 E+07$ & $4.98 E+07$ & & (1) 64.27 & & $M+1$ & & \\
\hline 41 & 760 & $1.18 \mathrm{E}+09$ & $1.19 E+09$ & $1.12 \mathrm{E}+09$ & & & 2333.95 & & & \\
\hline 42 & 761 & $5.40 \mathrm{E}+08$ & $5.59 \mathrm{E}+08$ & $5.01 \mathrm{E}+08$ & & ( 45.87 & & $M+1$ & & \\
\hline 43 & 762 & $1.62 \mathrm{E}+08$ & $1.68 \mathrm{E}+08$ & $1.48 \mathrm{E}+08$ & & & 29.83 & $M+1$ & & \\
\hline 44 & 768 & $4.67 E+06$ & $3.57 \mathrm{E}+06$ & $3.64 \mathrm{E}+06$ & & & & & & \\
\hline 45 & 769 & $5.25 \mathrm{E}+06$ & $5.73 E+06$ & $5.15 E+06$ & & 138.10 & & & & \\
\hline 46 & 770 & $9.44 E+06$ & $8.84 E+06$ & $9.07 E+06$ & & & ○ 170.13 & & & \\
\hline 47 & 771 & $4.24 \mathrm{E}+06$ & $4.29 E+06$ & $3.79 E+06$ & & 45.12 & & $M+1$ & & \\
\hline
\end{tabular}




\begin{tabular}{|c|c|c|c|c|c|c|c|c|c|c|}
\hline 4 & A & $\mathrm{B}$ & C & D & $\mathrm{J}$ & K & $\mathrm{L}$ & $\mathrm{M}$ & $\mathrm{N}$ & 0 \\
\hline 1 & $M / Z$ & \multicolumn{3}{|c|}{ January Areas } & lowest PC & $\mathrm{M}+1$ & 41.8 & $M+1$ & highest PC & 60.06 \\
\hline 2 & & & & & 676 & $M+1+20 \%$ & 61.8 & $M+1+20 \%$ & 898 & 80.06 \\
\hline 3 & & & & & lowest SM & $M+1$ & 43.25 & $M+1$ & highest SM & 60.9 \\
\hline 4 & & & & & 675 & $M+1+20 \%$ & 63.25 & $M+1+20 \%$ & 899 & 80.9 \\
\hline 5 & & Trial 1 & Trial 2 & Trial 3 & & & & & & \\
\hline 48 & 772 & $3.20 \mathrm{E}+07$ & $3.28 \mathrm{E}+07$ & $2.91 E+07$ & & & 761.79 & & & \\
\hline 49 & 773 & $1.45 E+07$ & $1.29 E+07$ & $1.28 \mathrm{E}+07$ & & 42.77 & & $M+1$ & & \\
\hline 50 & 774 & $2.34 \mathrm{E}+07$ & $2.84 \mathrm{E}+07$ & $2.41 \mathrm{E}+07$ & & & 190.12 & & & \\
\hline 51 & 775 & $1.09 \mathrm{E}+07$ & $1.09 \mathrm{E}+07$ & $1.06 \mathrm{E}+07$ & & 43.00 & & $M+1$ & & \\
\hline 52 & 776 & $4.24 \mathrm{E}+06$ & $3.82 E+06$ & $3.77 E+06$ & & & 36.54 & $M+1$ & & \\
\hline 53 & 778 & $2.00 E+07$ & $2.31 E+07$ & $2.00 E+07$ & & & & & & \\
\hline 54 & 779 & $1.23 E+07$ & 1.33E+07 & $1.09 \mathrm{E}+07$ & & ( 58.00 & & $M+1$ & & \\
\hline 55 & 780 & $8.24 \mathrm{E}+07$ & $8.46 \mathrm{E}+07$ & $7.75 \mathrm{E}+07$ & & & 671.85 & & & \\
\hline 56 & 781 & $3.49 E+07$ & $3.75 E+07$ & $3.35 E+07$ & & 43.30 & & $M+1$ & & \\
\hline 57 & 782 & $3.54 \mathrm{E}+07$ & $3.44 \mathrm{E}+07$ & $3.45 \mathrm{E}+07$ & & & 98.74 & & & \\
\hline 58 & 783 & $1.39 E+07$ & $1.36 \mathrm{E}+07$ & $1.29 E+07$ & & 38.69 & & $M+1$ & & \\
\hline 59 & 784 & $2.09 E+07$ & $2.01 E+07$ & $1.91 \mathrm{E}+07$ & & & 148.78 & & & \\
\hline 60 & 785 & $1.19 E+07$ & $1.09 \mathrm{E}+07$ & $9.69 \mathrm{E}+06$ & & 53.91 & & $M+1$ & & \\
\hline 61 & 786 & $9.09 E+07$ & $9.25 E+07$ & $9.03 E+07$ & & & 850.01 & & & \\
\hline 62 & 787 & $4.44 E+07$ & $4.73 E+07$ & $4.27 E+07$ & & 49.09 & & $M+1$ & & \\
\hline 63 & 788 & $5.16 \mathrm{E}+08$ & $5.21 E+08$ & $4.60 E+08$ & & & 1112.92 & & & \\
\hline 64 & 789 & $2.24 \mathrm{E}+08$ & $2.35 E+08$ & $2.01 \mathrm{E}+08$ & & 44.12 & & $M+1$ & & \\
\hline 65 & 790 & $5.56 \mathrm{E}+07$ & $5.82 \mathrm{E}+07$ & $5.15 E+07$ & & & 25.04 & $M+1$ & & \\
\hline 66 & 792 & $2.68 \mathrm{E}+07$ & $2.87 \mathrm{E}+07$ & $2.63 E+07$ & & & & & & \\
\hline 67 & 793 & $1.08 \mathrm{E}+07$ & $1.18 \mathrm{E}+07$ & $1.15 \mathrm{E}+07$ & & 41.56 & & $M+1$ & & \\
\hline 68 & 794 & $7.86 \mathrm{E}+06$ & $7.08 \mathrm{E}+06$ & $7.36 \mathrm{E}+06$ & & & 65.83 & $M+1$ & & \\
\hline 69 & 796 & $3.16 \mathrm{E}+06$ & $3.11 E+06$ & $3.39 \mathrm{E}+06$ & & & & & & \\
\hline 70 & 797 & $2.49 E+06$ & $2.01 \mathrm{E}+06$ & $2.03 E+06$ & & 67.70 & & $M+1$ & & \\
\hline 71 & 798 & $2.16 \mathrm{E}+06$ & $2.26 \mathrm{E}+06$ & $2.06 \mathrm{E}+06$ & & & 100.26 & & & \\
\hline 72 & 799 & $2.91 \mathrm{E}+06$ & $2.34 \mathrm{E}+06$ & $2.08 \mathrm{E}+06$ & & 113.05 & & & & \\
\hline 73 & 800 & $3.54 \mathrm{E}+06$ & $3.83 E+06$ & $3.18 \mathrm{E}+06$ & & & 146.04 & & & \\
\hline 74 & 801 & $1.73 E+06$ & $1.66 \mathrm{E}+06$ & $1.98 \mathrm{E}+06$ & & 51.55 & & $M+1$ & & \\
\hline 75 & 802 & $3.34 E+06$ & $3.52 E+06$ & $2.54 \mathrm{E}+06$ & & & 177.74 & & & \\
\hline 76 & 803 & $1.99 E+06$ & $1.66 \mathrm{E}+06$ & $1.71 \mathrm{E}+06$ & & ( 57.99 & & & & \\
\hline 77 & 804 & $3.15 E+07$ & $3.40 E+07$ & $2.86 \mathrm{E}+07$ & & & 1769.14 & & & \\
\hline 78 & 805 & $5.17 \mathrm{E}+07$ & $5.91 \mathrm{E}+07$ & $5.83 E+07$ & & 180.63 & & & & \\
\hline 79 & 806 & $7.62 E+08$ & $7.26 \mathrm{E}+08$ & $6.98 \mathrm{E}+08$ & & & 1299.93 & & & \\
\hline 80 & 807 & $3.73 E+08$ & $3.49 E+08$ & $3.41 \mathrm{E}+08$ & & 48.64 & & $M+1$ & & \\
\hline 81 & 808 & $1.49 E+08$ & $1.50 \mathrm{E}+08$ & $1.42 \mathrm{E}+08$ & & & 41.51 & $M+1$ & & \\
\hline 82 & 810 & $3.53 E+07$ & $3.70 \mathrm{E}+07$ & $3.68 \mathrm{E}+07$ & & & & & & \\
\hline 83 & 811 & $1.48 \mathrm{E}+07$ & $1.54 \mathrm{E}+07$ & $1.54 \mathrm{E}+07$ & & 41.87 & & $M+1$ & & \\
\hline 84 & 812 & $1.33 E+07$ & 1.24E+07 & $1.29 E+07$ & & & 84.57 & & & \\
\hline 85 & 813 & $2.18 \mathrm{E}+07$ & $2.42 \mathrm{E}+07$ & $2.09 \mathrm{E}+07$ & & 174.10 & & & & \\
\hline 86 & 814 & $4.23 E+06$ & $3.47 E+06$ & $3.28 \mathrm{E}+06$ & & & & & & \\
\hline 87 & 814 & $2.57 E+07$ & $2.89 E+07$ & $2.85 E+07$ & & & 124.29 & & & \\
\hline 88 & 815 & $5.01 E+06$ & $4.78 \mathrm{E}+06$ & $3.96 \mathrm{E}+06$ & & 125.64 & & & & \\
\hline 89 & 815 & $1.06 \mathrm{E}+07$ & $1.22 \mathrm{E}+07$ & $9.73 E+06$ & & ( 39.21 & & $M+1$ & & \\
\hline | n n & $\cdots$ & $\cdots$ & $\cdots$ & $-\cdots-\cdots$ & & & & $\cdots$ & & \\
\hline
\end{tabular}




\begin{tabular}{|c|c|c|c|c|c|c|c|c|c|c|}
\hline 4 & A & $\mathrm{B}$ & $\mathrm{C}$ & D & $J$ & $\mathrm{~K}$ & $\mathrm{~L}$ & $\mathrm{M}$ & $\mathrm{N}$ & 0 \\
\hline 1 & $\mathrm{M} / \mathrm{Z}$ & \multicolumn{3}{|c|}{ January Areas } & lowest PC & $\mathrm{M}+1$ & 41.8 & $M+1$ & highest PC & 60.06 \\
\hline 2 & & & & & 676 & $M+1+20 \%$ & 61.8 & $M+1+20 \%$ & 898 & 80.06 \\
\hline 3 & & & & & lowest SM & $\mathrm{M}+1$ & 43.25 & $M+1$ & highest SM & 60.9 \\
\hline 4 & & & & & 675 & $M+1+20 \%$ & 63.25 & $M+1+20 \%$ & 899 & 80.9 \\
\hline 5 & & Trial 1 & Trial 2 & Trial 3 & & & & & & \\
\hline 90 & 816 & $2.28 \mathrm{E}+06$ & $2.59 \mathrm{E}+06$ & $2.44 \mathrm{E}+06$ & & & 53.81 & $M+1$ & & \\
\hline 91 & 816 & $1.98 \mathrm{E}+07$ & $2.28 \mathrm{E}+07$ & $1.78 \mathrm{E}+07$ & & & 185.40 & & & \\
\hline 92 & 817 & $1.04 \mathrm{E}+07$ & $1.24 \mathrm{E}+07$ & $9.44 \mathrm{E}+06$ & & 53.32 & & $M+1$ & & \\
\hline 93 & 818 & $2.22 \mathrm{E}+07$ & $2.41 E+07$ & $2.37 E+07$ & & & 219.71 & & & \\
\hline 94 & 819 & $1.31 \mathrm{E}+07$ & $1.39 \mathrm{E}+07$ & $1.16 \mathrm{E}+07$ & & 55.32 & & $M+1$ & & \\
\hline 95 & 820 & $2.41 \mathrm{E}+07$ & $2.49 E+07$ & $2.38 \mathrm{E}+07$ & & & 189.35 & & & \\
\hline 96 & 821 & $9.27 E+06$ & $9.67 \mathrm{E}+06$ & $9.57 \mathrm{E}+06$ & & 39.13 & & $M+1$ & & \\
\hline 97 & 822 & $4.44 \mathrm{E}+06$ & $4.37 E+06$ & $4.60 \mathrm{E}+06$ & & & 47.06 & $M+1$ & & \\
\hline 98 & 826 & $2.14 \mathrm{E}+06$ & $1.75 \mathrm{E}+06$ & $1.61 E+06$ & & & & & & \\
\hline 99 & 827 & $2.47 E+06$ & $2.54 \mathrm{E}+06$ & $1.83 E+06$ & & 124.65 & & & & \\
\hline 100 & 828 & $5.87 E+06$ & $5.85 E+06$ & $4.73 E+06$ & & & 242.22 & & & \\
\hline 101 & 829 & $3.54 \mathrm{E}+06$ & $4.50 E+06$ & $3.11 E+06$ & & 67.68 & & $M+1$ & & \\
\hline 102 & 830 & $7.38 \mathrm{E}+06$ & $8.24 \mathrm{E}+06$ & $7.56 \mathrm{E}+06$ & & & 211.47 & & & \\
\hline 103 & 831 & $5.90 \mathrm{E}+06$ & $6.76 \mathrm{E}+06$ & $4.85 E+06$ & & 75.36 & & & & \\
\hline 104 & 832 & $1.05 E+08$ & $1.07 E+08$ & $9.70 \mathrm{E}+07$ & & & 1788.70 & & & \\
\hline 105 & 833 & $7.18 \mathrm{E}+07$ & $8.53 E+07$ & $7.55 \mathrm{E}+07$ & & 75.30 & & & & \\
\hline 106 & 834 & $1.01 \mathrm{E}+09$ & $1.07 \mathrm{E}+09$ & $9.59 \mathrm{E}+08$ & & & 1311.86 & & & \\
\hline 107 & 835 & $4.97 E+08$ & $5.33 E+08$ & $4.67 E+08$ & & 49.18 & & $M+1$ & & \\
\hline 108 & 836 & $1.38 \mathrm{E}+08$ & $1.53 E+08$ & $1.34 \mathrm{E}+08$ & & & 28.45 & $M+1$ & & \\
\hline 109 & 842 & $4.92 E+06$ & $6.11 E+06$ & $4.20 \mathrm{E}+06$ & & & & & & \\
\hline 110 & 842 & $1.99 \mathrm{E}+07$ & $2.28 \mathrm{E}+07$ & $1.72 \mathrm{E}+07$ & & & & & & \\
\hline 111 & 843 & $1.17 \mathrm{E}+07$ & $1.06 \mathrm{E}+07$ & $8.78 \mathrm{E}+06$ & & 206.70 & & & & \\
\hline 112 & 843 & $9.91 E+06$ & $1.08 \mathrm{E}+07$ & $8.42 E+06$ & & 48.75 & & $M+1$ & & \\
\hline 113 & 844 & $2.88 \mathrm{E}+06$ & $3.49 E+06$ & $2.50 E+06$ & & & 28.66 & $M+1$ & & \\
\hline 114 & 844 & $6.43 E+06$ & $5.77 \mathrm{E}+06$ & $4.76 \mathrm{E}+06$ & & & 58.27 & $M+1$ & & \\
\hline 115 & 846 & $3.64 \mathrm{E}+06$ & $3.14 \mathrm{E}+06$ & $3.72 \mathrm{E}+06$ & & & & & & \\
\hline 116 & 847 & $1.76 \mathrm{E}+06$ & $2.01 E+06$ & $1.68 \mathrm{E}+06$ & & 52.52 & & $M+1$ & & \\
\hline 117 & 848 & $3.42 \mathrm{E}+06$ & $3.39 E+06$ & $2.51 \mathrm{E}+06$ & & & 170.76 & & & \\
\hline 118 & 850 & $2.57 E+06$ & $2.79 \mathrm{E}+06$ & $2.08 \mathrm{E}+06$ & & & & & & \\
\hline 119 & 851 & $4.72 E+06$ & $5.25 \mathrm{E}+06$ & $4.37 E+06$ & & 193.83 & & & & \\
\hline 120 & 852 & $1.15 \mathrm{E}+07$ & $1.22 \mathrm{E}+07$ & $1.05 \mathrm{E}+07$ & & & 238.82 & & & \\
\hline 121 & 853 & $6.43 E+06$ & $6.81 \mathrm{E}+06$ & $5.95 \mathrm{E}+06$ & & 56.11 & & $M+1$ & & \\
\hline 122 & 854 & $9.32 \mathrm{E}+06$ & $1.01 \mathrm{E}+07$ & $8.36 \mathrm{E}+06$ & & & 144.72 & & & \\
\hline 123 & 855 & $4.22 E+06$ & 4.77E+06 & $4.12 E+06$ & & 47.22 & & $M+1$ & & \\
\hline 124 & 856 & $4.97 E+06$ & $4.82 E+06$ & $4.52 E+06$ & & & 109.53 & & & \\
\hline 125 & 856 & $1.78 \mathrm{E}+06$ & $1.79 \mathrm{E}+06$ & $1.23 E+06$ & & & & & & \\
\hline 126 & 857 & $2.26 \mathrm{E}+06$ & $2.13 E+06$ & $2.13 E+06$ & & 45.66 & & $M+1$ & & \\
\hline 127 & 858 & 4.57E+06 & $4.19 E+06$ & $4.29 E+06$ & & & 199.97 & & & \\
\hline 128 & 859 & $3.19 E+06$ & $3.24 \mathrm{E}+06$ & $2.44 \mathrm{E}+06$ & & (1) $\quad 67.92$ & & $M+1$ & & \\
\hline 129 & 860 & $2.65 E+07$ & $2.87 E+07$ & $2.63 E+07$ & & & 931.48 & & & \\
\hline 130 & 861 & $1.42 \mathrm{E}+07$ & $1.49 \mathrm{E}+07$ & $1.30 \mathrm{E}+07$ & & 51.64 & & $M+1$ & & \\
\hline 131 & 862 & $2.58 \mathrm{E}+07$ & $2.72 E+07$ & $2.28 \mathrm{E}+07$ & & & 179.92 & & & \\
\hline
\end{tabular}




\begin{tabular}{|c|c|c|c|c|c|c|c|c|c|c|}
\hline 4 & A & B & $\mathrm{C}$ & D & $J$ & $\mathrm{~K}$ & L & $\mathrm{M}$ & $\mathrm{N}$ & 0 \\
\hline 1 & $\mathrm{M} / \mathrm{Z}$ & \multicolumn{3}{|c|}{ January Areas } & lowest PC & M+1 & 41.8 & $M+1$ & highest PC & 60.06 \\
\hline 2 & & & & & 676 & $M+1+20 \%$ & 61.8 & $M+1+20 \%$ & 898 & 80.06 \\
\hline 3 & & & & & lowest SM & $M+1$ & 43.25 & $M+1$ & highest SM & 60.9 \\
\hline 4 & & & & & 675 & $M+1+20 \%$ & 63.25 & $M+1+20 \%$ & 899 & 80.9 \\
\hline 5 & & Trial 1 & Trial 2 & Trial 3 & & & & & & \\
\hline 132 & 863 & $1.16 \mathrm{E}+07$ & $1.26 \mathrm{E}+07$ & $9.69 \mathrm{E}+06$ & & 44.67 & & $M+1$ & & \\
\hline 133 & 864 & $5.40 \mathrm{E}+06$ & $4.77 E+06$ & $4.68 \mathrm{E}+06$ & & & 44.18 & $M+1$ & & \\
\hline 134 & 868 & $4.22 \mathrm{E}+06$ & $4.19 E+06$ & $3.16 \mathrm{E}+06$ & & & & & & \\
\hline 135 & 869 & $1.73 E+06$ & $2.39 E+06$ & $1.66 \mathrm{E}+06$ & & 50.11 & & $M+1$ & & \\
\hline 136 & 870 & $2.73 E+07$ & $3.12 E+07$ & $2.73 E+07$ & & & 1511.09 & & & \\
\hline 137 & 871 & $1.36 \mathrm{E}+07$ & $1.70 \mathrm{E}+07$ & $1.28 \mathrm{E}+07$ & & 50.32 & & $M+1$ & & \\
\hline 138 & 872 & $5.57 \mathrm{E}+06$ & $7.41 E+06$ & $5.42 E+06$ & & & 42.36 & $M+1$ & & \\
\hline 139 & 877 & $8.11 E+06$ & $9.17 \mathrm{E}+06$ & $9.22 \mathrm{E}+06$ & & & & & & \\
\hline 140 & 878 & $3.67 E+08$ & $3.92 E+08$ & $3.69 E+08$ & & & 4269.95 & & & \\
\hline 141 & 879 & $1.96 \mathrm{E}+08$ & $2.18 \mathrm{E}+08$ & $2.05 E+08$ & & 54.85 & & $M+1$ & & \\
\hline 142 & 880 & $6.12 E+07$ & $6.91 E+07$ & $6.32 \mathrm{E}+07$ & & & 31.23 & $M+1$ & & \\
\hline 143 & 887 & $2.54 \mathrm{E}+06$ & $2.39 E+06$ & $1.93 E+06$ & & & & & & \\
\hline 144 & 888 & $3.31 E+06$ & $3.94 \mathrm{E}+06$ & $2.91 \mathrm{E}+06$ & & & 148.67 & & & \\
\hline 145 & 889 & $2.29 \mathrm{E}+06$ & $2.49 E+06$ & $1.66 \mathrm{E}+06$ & & ( 62.97 & & $M+1$ & & \\
\hline 146 & 896 & $3.39 E+06$ & $5.75 E+06$ & $3.49 E+06$ & & & & & & \\
\hline 147 & 897 & $2.16 \mathrm{E}+06$ & $2.46 E+06$ & $1.81 \mathrm{E}+06$ & & 52.76 & & $M+1$ & & \\
\hline 148 & 898 & $9.74 \mathrm{E}+06$ & $1.08 \mathrm{E}+07$ & $8.19 E+06$ & & & 446.94 & & & \\
\hline 149 & 899 & $3.37 E+06$ & $5.12 \mathrm{E}+06$ & $4.24 E+06$ & & 44.67 & & $M+1$ & & \\
\hline
\end{tabular}

Figure 6:9. $M+1$ calculations for goldfish retina membrane PC and SM species in January done in Excel.

The peaks highlighted in green in Figure 6:9 are the peaks that are below the LOQ. However, these peaks are retained as phospholipid candidates as they are below the LOQ in 1 out of 3 technical trials.

The last task before phospholipid quantification was subtracting all possible PC $M+1$ peaks from SM peaks as the proportion of $P C M+1$ in $S M$ peak is around $50 \%$. This procedure is shown in Figure 6:10. Also, SM M+1 peaks were subtracted from PC peaks, this data not shown here. These new area values for PCs and SMs were used in quantification calculations. The contribution from $M+2$ peaks is not as significant as from $\mathrm{M}+1$ peaks, so they were not subtracted from any phospholipid area values. 


\begin{tabular}{|c|c|c|c|c|c|c|c|c|c|c|c|c|c|c|c|c|c|c|}
\hline sun & & 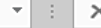 & $\checkmark$ & $=C 7$ & $7-\left(\mathrm{C} 6^{*} \$ \mathrm{~J} 6\right)$ & & & & & & & & & & & & & \\
\hline$\Delta$ & A & B & c & D & E & $\mathrm{F}$ & G & H & \multirow{3}{*}{$\begin{array}{c}1 \\
\%(m+1)\end{array}$} & J & \multirow[t]{3}{*}{$\mathrm{K}$} & \multirow{3}{*}{$\frac{L}{M / Z}$} & M & $\mathrm{N}$ & 0 & P & \multicolumn{2}{|l|}{$\frac{\mathrm{Q}}{\text { July Areas }}$} \\
\hline 1 & & $M / Z$ & \multicolumn{3}{|c|}{ January Areas } & \multicolumn{3}{|c|}{ July Areas } & & & & & \multicolumn{3}{|c|}{ January Areas } & \multicolumn{3}{|c|}{ July Areas } \\
\hline 2 & & & & & & & & & & & & & & & & & & \\
\hline 3 & & & Trial 1 & Trial 2 & Trial 3 & Trial 1 & Trial 2 & Trial 3 & & & & & Trial 1 & Trial 2 & Trial 3 & Trial 1 & Trial 2 & Trial 3 \\
\hline 4 & & 675 & $1.49 \mathrm{E}+07$ & $7.46 \mathrm{E}+06$ & $2.39 \mathrm{E}+06$ & $8.79 E+05$ & $6.53 E+06$ & 7.83E+06 & & & & 675 & $1.49 \mathrm{E}+07$ & $7.46 \mathrm{E}+06$ & $2.39 \mathrm{E}+06$ & $8.79 E+05$ & $6.53 E+06$ & 7.83E+06 \\
\hline 5 & & 689 & $1.88 \mathrm{E}+06$ & $1.91 E+06$ & 1.23E+06 & 1.13E+06 & 1.21E+06 & 1.13E+06 & & & & 689 & $1.88 \mathrm{E}+06$ & $1.91 E+06$ & $1.23 \mathrm{E}+06$ & $1.13 E+06$ & $1.21 \mathrm{E}+06$ & 1.13E+06 \\
\hline 6 & & 702 & $4.77 \mathrm{E}+05$ & $5.02 E+05$ & $6.28 E+05$ & $6.53 E+05$ & $3.26 \mathrm{E}+05$ & $5.52 E+05$ & 44.0656 & 0.4407 & & 703 & $=\mathrm{C} 7-(\mathrm{C} 6 * \mathrm{~S}$ & $9.01 E+07$ & 7.41E+07 & $4.78 E+07$ & $6.75 E+07$ & $5.25 E+07$ \\
\hline 7 & & 703 & $7.78 \mathrm{E}+07$ & $9.03 E+07$ & 7.44E+07 & $4.81 E+07$ & $6.77 E+07$ & $5.28 E+07$ & & & & 717 & $3.19 \mathrm{E}+06$ & $3.85 E+06$ & $3.02 E+06$ & $2.60 \mathrm{E}+06$ & $2.96 \mathrm{E}+06$ & 2.00E+06 \\
\hline 8 & & 716 & $6.15 E+06$ & $5.75 \mathrm{E}+06$ & $5.05 E+06$ & $5.20 \mathrm{E}+06$ & $5.73 E+06$ & 5.57E+06 & 45.2081 & 0.4521 & & 731 & $3.20 \mathrm{E}+07$ & $2.79 E+07$ & $2.22 \mathrm{E}+07$ & $2.52 E+07$ & $2.80 E+07$ & $2.48 E+07$ \\
\hline 9 & & 717 & $5.98 E+06$ & $6.45 E+06$ & $5.30 \mathrm{E}+06$ & 4.95E+06 & $5.55 E+06$ & 4.52E+06 & & & & 741 & $2.85 E+07$ & $3.24 E+07$ & $2.60 \mathrm{E}+07$ & $1.26 \mathrm{E}+07$ & $1.60 E+07$ & $1.38 \mathrm{E}+07$ \\
\hline 10 & & 730 & $1.16 E+07$ & $1.19 E+07$ & $1.11 E+07$ & $8.41 E+06$ & $9.72 E+06$ & 9.64E+06 & 46.3505 & 0.4635 & & 769 & $2.92 E+06$ & $3.95 E+06$ & $3.34 E+06$ & $3.10 E+06$ & $3.03 E+06$ & $2.88 \mathrm{E}+06$ \\
\hline 11 & & 731 & $3.74 E+07$ & $3.35 E+07$ & $2.74 E+07$ & $2.91 E+07$ & $3.25 E+07$ & $2.93 E+07$ & & & & 799 & $1.79 E+06$ & $1.16 E+06$ & $1.01 \mathrm{E}+06$ & $1.34 E+06$ & $1.06 \mathrm{E}+06$ & 1.16E+06 \\
\hline 12 & & 740 & $5.78 E+05$ & 4.27E+05 & $5.78 E+05$ & $3.01 E+05$ & $3.26 E+05$ & 4.02E+05 & 47.4530 & 0.4745 & & 803 & $2.52 E+05$ & $-1.78 E+05$ & $3.86 \mathrm{E}+05$ & $5.08 E+05$ & $9.00 E+05$ & 1.07E+06 \\
\hline 13 & & 741 & $2.88 \mathrm{E}+07$ & $3.26 E+07$ & $2.63 E+07$ & $1.28 \mathrm{E}+07$ & $1.61 E+07$ & $1.40 E+07$ & & & & 805 & $3.49 E+07$ & $4.10 E+07$ & $4.31 E+07$ & $4.18 E+07$ & $4.23 E+07$ & $3.85 E+07$ \\
\hline 14 & & 768 & $4.67 E+06$ & $3.57 \mathrm{E}+06$ & $3.64 E+06$ & $3.06 E+06$ & $3.14 E+06$ & $3.39 E+06$ & 49.7379 & 0.4974 & & 813 & $1.47 \mathrm{E}+07$ & $1.76 \mathrm{E}+07$ & $1.41 \mathrm{E}+07$ & $1.12 \mathrm{E}+07$ & $1.17 \mathrm{E}+07$ & $1.28 \mathrm{E}+07$ \\
\hline 15 & & 769 & $5.25 E+06$ & $5.73 E+06$ & $5.15 E+06$ & 4.62E+06 & $4.60 E+06$ & 4.57E+06 & & & & 815 & $2.76 \mathrm{E}+06$ & $2.94 E+06$ & $2.21 E+06$ & $1.88 \mathrm{E}+06$ & $1.48 \mathrm{E}+06$ & 2.05E+06 \\
\hline 16 & & 798 & $2.16 \mathrm{E}+06$ & $2.26 \mathrm{E}+06$ & $2.06 \mathrm{E}+06$ & $1.63 E+06$ & $1.73 E+06$ & $1.43 E+06$ & 52.0428 & 0.5204 & & 827 & $1.30 \mathrm{E}+06$ & $1.58 \mathrm{E}+06$ & $9.60 \mathrm{E}+05$ & $1.16 \mathrm{E}+06$ & $1.65 \mathrm{E}+06$ & $1.59 \mathrm{E}+06$ \\
\hline 17 & & 799 & $2.91 E+06$ & $2.34 E+06$ & $2.08 \mathrm{E}+06$ & 2.19E+06 & $1.96 \mathrm{E}+06$ & $1.91 \mathrm{E}+06$ & & & & 831 & $1.81 \mathrm{E}+06$ & $2.19 E+06$ & $6.61 E+05$ & $1.70 \mathrm{E}+06$ & $2.48 \mathrm{E}+06$ & $2.21 \mathrm{E}+06$ \\
\hline 18 & & 802 & $3.34 E+06$ & $3.52 E+06$ & 2.54E+06 & $2.60 \mathrm{E}+06$ & $2.03 E+06$ & 2.19E+06 & 52.0828 & 0.5208 & & 833 & $1.35 E+07$ & $2.61 E+07$ & $2.18 \mathrm{E}+07$ & $2.20 E+07$ & $2.02 E+07$ & $1.68 \mathrm{E}+07$ \\
\hline 19 & & 803 & 1.99E+06 & $1.66 \mathrm{E}+06$ & $1.71 E+06$ & $1.86 \mathrm{E}+06$ & $1.96 \mathrm{E}+06$ & $2.21 \mathrm{E}+06$ & & & & 843 & $8.99 \mathrm{E}+06$ & 7.18E+06 & $6.45 E+06$ & 4.48E+06 & $2.80 \mathrm{E}+06$ & $3.74 E+06$ \\
\hline 20 & & 804 & $3.15 E+07$ & $3.40 E+07$ & 2.86E+07 & $2.41 E+07$ & $2.93 E+07$ & $2.65 E+07$ & 53.1053 & 0.5311 & & 851 & $3.27 \mathrm{E}+06$ & $3.67 E+06$ & $3.19 E+06$ & $2.49 E+06$ & $2.66 E+06$ & $2.91 E+06$ \\
\hline 21 & & 805 & 5.17E+07 & $5.91 E+07$ & 5.83E+07 & 5.46E+07 & $5.79 E+07$ & $5.26 \mathrm{E}+07$ & & & & 877 & $7.62 E+06$ & $8.58 \mathrm{E}+06$ & $8.68 \mathrm{E}+06$ & $8.98 \mathrm{E}+06$ & $1.15 E+07$ & $1.08 \mathrm{E}+07$ \\
\hline 22 & & 812 & $1.33 E+07$ & $1.24 E+07$ & $1.29 \mathrm{E}+07$ & 1.19E+07 & $1.31 E+07$ & $1.38 E+07$ & 53.1853 & 0.5319 & & 887 & $1.58 \mathrm{E}+06$ & $1.23 E+06$ & $1.27 \mathrm{E}+06$ & $3.19 E+05$ & $2.51 E+05$ & $1.05 E+05$ \\
\hline 23 & & 813 & $2.18 E+07$ & $2.42 E+07$ & $2.09 E+07$ & $1.75 E+07$ & 1.87E+07 & $2.01 E+07$ & & & & & & & & & & \\
\hline 24 & & 814 & $4.23 E+06$ & 3.47E+06 & 3.28E+06 & $2.57 E+06$ & $2.58 \mathrm{E}+06$ & 1.79E+06 & 53.2053 & 0.5321 & & & & & & & & \\
\hline 25 & & 815 & $5.01 E+06$ & $4.78 E+06$ & $3.96 \mathrm{E}+06$ & $3.25 \mathrm{E}+06$ & $2.86 E+06$ & $3.00 E+06$ & & & & & & & & & & \\
\hline 26 & & 826 & $2.14 E+06$ & $1.75 E+06$ & $1.61 E+06$ & $9.14 E+05$ & 1.44E+06 & 1.13E+06 & 54.3277 & 0.5433 & & & & & & & & \\
\hline 27 & & 827 & 2.47E+06 & $2.54 \mathrm{E}+06$ & 1.83E+06 & $1.66 \mathrm{E}+06$ & 2.44E+06 & $2.21 \mathrm{E}+06$ & & & & & & & & & & \\
\hline 28 & & 830 & $7.38 \mathrm{E}+06$ & $8.24 E+06$ & 7.56E+06 & $6.96 \mathrm{E}+06$ & $7.58 E+06$ & $9.12 E+06$ & 55.3702 & 0.5537 & & & & & & & & \\
\hline 29 & & 831 & $5.90 E+06$ & $6.76 \mathrm{E}+06$ & 4.85E+06 & $5.55 \mathrm{E}+06$ & $6.68 \mathrm{E}+06$ & 7.26E+06 & & & & & & & & & & \\
\hline 30 & & 832 & $1.05 E+08$ & $1.07 E+08$ & 9.70E+07 & $8.91 E+07$ & $1.00 E+08$ & $1.02 \mathrm{E}+08$ & 55.3902 & 0.5539 & & & & & & & & \\
\hline 31 & & 833 & 7.18E+07 & $8.53 E+07$ & $7.55 E+07$ & $7.13 E+07$ & $7.57 E+07$ & 7.34E+07 & & & & & & & & & & \\
\hline 32 & & 842 & $4.92 E+06$ & $6.11 \mathrm{E}+06$ & $4.20 E+06$ & $3.38 \mathrm{E}+06$ & $2.90 \mathrm{E}+06$ & $3.69 E+06$ & 55.4902 & 0.5549 & & & & & & & & \\
\hline 33 & & 843 & $1.17 E+07$ & $1.06 \mathrm{E}+07$ & $8.78 E+06$ & $6.36 \mathrm{E}+06$ & 4.41E+06 & $5.79 E+06$ & & & & & & & & & & \\
\hline 34 & & 850 & $2.57 E+06$ & $2.79 \mathrm{E}+06$ & $2.08 E+06$ & 1.33E+06 & $1.68 \mathrm{E}+06$ & $2.26 \mathrm{E}+06$ & 56.5727 & 0.5657 & & & & & & & & \\
\hline 35 & & 851 & 4.72E+06 & $5.25 \mathrm{E}+06$ & 4.37E+06 & $3.24 E+06$ & $3.62 E+06$ & 4.19E+06 & & & & & & & & & & \\
\hline 36 & & 876 & $8.29 E+05$ & $1.00 \mathrm{E}+06$ & 9.04E+05 & $2.76 E+05$ & $4.52 E+05$ & $2.77 E+05$ & 58.8376 & 0.5884 & & & & & & & & \\
\hline 37 & & 877 & $8.11 E+06$ & 9.17E+06 & $9.22 E+06$ & $9.14 E+06$ & $1.18 E+07$ & $1.10 \mathrm{E}+07$ & & & & & & & & & & \\
\hline 38 & & 886 & $1.63 E+06$ & 1.97E+06 & $1.13 E+06$ & $1.63 E+06$ & $1.41 \mathrm{E}+06$ & $1.53 E+06$ & 58.9376 & 0.5894 & & & & & & & & \\
\hline 39 & & 887 & $2.54 \mathrm{E}+06$ & $2.39 \mathrm{E}+06$ & $1.93 \mathrm{E}+06$ & $1.28 \mathrm{E}+06$ & $1.08 \mathrm{E}+06$ & $1.01 E+06$ & & & & & & & & & & \\
\hline in & & & & & & & & & & & & & & & & & & \\
\hline
\end{tabular}

Figure 6:10. $P C M+1$ contribution subtraction from $S M$ peaks.

Student's t-test: Figures 6:11-6:13 are pieces of one continuous Excel sheet which ultimately show how the phospholipids are changing between the seasons (January versus July). Figure $6: 13$ shows the fold changes and $p$-values at $\alpha=0.05$ between January and July of all the PE species found in goldfish retina membranes. The dark orange indicates values that are above 0.05 and do not have significant changes between the seasons. Green indicates values below 0.05; these PEs have significant changes between the seasons. It can be observed that 6 PEs have been found to decrease in July significantly when compared to January values using t-test statistics found in Excel with 
two-tailed distribution and with two-sample equal variance. The two-tailed distribution considers that the phospholipids could be either increasing or decreasing when going from January to July in goldfish retina membranes. The two-sample equal variance t-test assumes two things: the distributions of two populations are normal and the variances of the two distributions are the same. It is difficult to test for normality for a sample size that has less than 30 points $^{8}$. The t-test is used when the sample size is less than or equal to 30 and the population variance and/or standard deviation are not known ${ }^{9}$. An f-test could be used to determine whether two variances of two means are equal or not; this can help to choose proper statistics for comparing two means ${ }^{10}$. It was determined that $54 \%$ of PEs have equal variances between January and July means. The equal variance t-test was chosen under the assumption that variances are expected to be similar as lipidomic profiles are not assumed to change a lot between the seasons, especially as other variables are kept quite constant in the animals.

False discovery rate (FDR): The final statistical method that was performed on the lowresolution data was reducing a chance of finding false positives; that is finding statistically significant changes between the seasons when they could be not statistically significant (this is called a type I error). There are several methods that control false discovery rate. False discovery methods have gained popularity in scientific statistics compared to familywise error rates methods (FWER) that were more popular before the 1990s in multiple comparison testings ${ }^{11}$. FWER methods are more stringent compared to FDR methods as there is a possibility to dismiss values that are statistically significant because the FWER 


\begin{tabular}{|c|c|c|c|c|c|c|c|c|c|c|c|c|c|c|c|c|}
\hline 4 & A & $\mathrm{B}$ & $\mathrm{C}$ & D & $\mathrm{E}$ & $\mathrm{F}$ & G & $\mathrm{H}$ & 1 & $J$ & $\mathrm{~K}$ & L & $\mathrm{M}$ & $\mathrm{N}$ & 0 & $\mathrm{P}$ \\
\hline 1 & $M / Z$ & \multicolumn{3}{|c|}{ Areas } & & \multicolumn{3}{|c|}{ Retention Times (mins) } & & \multicolumn{3}{|c|}{ Standardized Area } & & \multicolumn{3}{|c|}{ Standardized Retention Time } \\
\hline 2 & & & & & & & & & & & & & & & & \\
\hline 3 & & Trial 1 & Trial 2 & Trial 3 & & Trial 1 & Trial 2 & Trial 3 & & Trial 1 & Trial 2 & Trial 3 & & Trial 1 & Trial 2 & Trial 3 \\
\hline 4 & 454 & $7.31 \mathrm{E}+07$ & $7.68 \mathrm{E}+07$ & $7.95 \mathrm{E}+07$ & & 14.10 & 14.13 & 14.11 & & & & & & & & \\
\hline 5 & & & & & & & & & & & & & & & & \\
\hline 6 & & & & & & & & & & & & & & & & \\
\hline 7 & 674 & $1.71 E+06$ & $1.66 E+06$ & $1.13 E+06$ & & 24.88 & 25.06 & 24.92 & & 0.02 & 0.02 & 0.01 & & 10.78 & 10.93 & 10.81 \\
\hline 8 & 688 & $1.36 \mathrm{E}+06$ & 2.13E+06 & 1.66E+06 & & 23.63 & 23.91 & 23.85 & & 0.02 & 0.03 & 0.02 & & 9.53 & 9.78 & 9.74 \\
\hline 9 & 690 & $3.89 E+06$ & $4.80 \mathrm{E}+06$ & $4.52 E+06$ & & 24.33 & 24.30 & 24.28 & & 0.05 & 0.06 & 0.06 & & 10.23 & 10.17 & 10.17 \\
\hline 10 & 702 & $5.83 E+06$ & $4.45 \mathrm{E}+06$ & $5.75 \mathrm{E}+06$ & & 25.41 & 25.65 & 25.59 & & 0.08 & 0.06 & 0.07 & & 11.31 & 11.52 & 11.48 \\
\hline 11 & 704 & $2.21 E+06$ & $2.08 \mathrm{E}+06$ & $1.96 \mathrm{E}+06$ & & 25.57 & 25.89 & 25.71 & & 0.03 & 0.03 & 0.02 & & 11.47 & 11.76 & 11.60 \\
\hline 12 & 716 & $5.60 \mathrm{E}+06$ & $7.16 \mathrm{E}+06$ & $7.83 E+06$ & & 24.58 & 24.75 & 24.67 & & 0.08 & 0.09 & 0.10 & & 10.48 & 10.62 & 10.56 \\
\hline 13 & 718 & $1.58 \mathrm{E}+07$ & $1.55 \mathrm{E}+07$ & $1.87 \mathrm{E}+07$ & & 25.18 & 25.38 & 25.31 & & 0.22 & 0.20 & 0.24 & & 11.08 & 11.25 & 11.20 \\
\hline 14 & 728 & $3.14 \mathrm{E}+06$ & $2.96 \mathrm{E}+06$ & $3.26 E+06$ & & 26.06 & 26.17 & 26.03 & & 0.04 & 0.04 & 0.04 & & 11.96 & 12.04 & 11.92 \\
\hline 15 & 730 & 4.34E+06 & 4.17E+06 & $3.97 \mathrm{E}+06$ & & 26.15 & 26.41 & 26.11 & & 0.06 & 0.05 & 0.05 & & 12.05 & 12.28 & 12.00 \\
\hline 16 & 736 & 1.26E+06 & 1.26E+06 & 1.46E+06 & & 23.86 & 24.02 & 23.95 & & 0.02 & 0.02 & 0.02 & & 9.76 & 9.89 & 9.84 \\
\hline 17 & 738 & $2.69 E+06$ & $3.92 \mathrm{E}+06$ & $2.74 E+06$ & & 24.27 & 24.32 & 24.48 & & 0.04 & 0.05 & 0.03 & & 10.17 & 10.19 & 10.37 \\
\hline 18 & 740 & $4.80 E+06$ & $5.10 \mathrm{E}+06$ & $5.27 \mathrm{E}+06$ & & 24.85 & 24.87 & 25.03 & & 0.07 & 0.07 & 0.07 & & 10.75 & 10.74 & 10.92 \\
\hline 19 & 744 & $9.22 \mathrm{E}+06$ & $1.08 \mathrm{E}+07$ & $9.79 \mathrm{E}+06$ & & 25.44 & 25.58 & 25.61 & & 0.13 & 0.14 & 0.12 & & 11.34 & 11.45 & 11.50 \\
\hline 20 & 746 & $1.85 \mathrm{E}+07$ & $1.82 \mathrm{E}+07$ & $1.63 E+07$ & & 26.14 & 26.32 & 26.18 & & 0.25 & 0.24 & 0.21 & & 12.04 & 12.19 & 12.07 \\
\hline 21 & 750 & 1.27E+07 & $1.26 \mathrm{E}+07$ & $1.35 E+07$ & & 25.35 & 25.51 & 25.49 & & 0.17 & 0.16 & 0.17 & & 11.25 & 11.38 & 11.38 \\
\hline 22 & 762 & $2.26 \mathrm{E}+06$ & $3.37 E+06$ & $3.04 E+06$ & & 24.05 & 24.14 & 24.28 & & 0.03 & 0.04 & 0.04 & & 9.95 & 10.01 & 10.17 \\
\hline 23 & 764 & $6.22 \mathrm{E}+07$ & $7.02 \mathrm{E}+07$ & $6.65 E+07$ & & 24.88 & 24.97 & 24.97 & & 0.85 & 0.91 & 0.84 & & 10.78 & 10.84 & 10.86 \\
\hline 24 & 766 & $1.84 \mathrm{E}+07$ & $2.31 E+07$ & $2.03 E+07$ & & 25.19 & 25.29 & 25.27 & & 0.25 & 0.30 & 0.26 & & 11.09 & 11.16 & 11.16 \\
\hline 25 & 768 & $2.73 E+07$ & $3.10 \mathrm{E}+07$ & $2.85 E+07$ & & 25.80 & 25.95 & 25.90 & & 0.37 & 0.40 & 0.36 & & 11.70 & 11.82 & 11.79 \\
\hline 26 & 772 & $2.69 E+06$ & $3.52 E+06$ & $2.46 E+06$ & & 26.17 & 26.25 & 26.45 & & 0.04 & 0.05 & 0.03 & & 12.07 & 12.12 & 12.34 \\
\hline 27 & 774 & 5.30E+06 & $6.43 E+06$ & $5.32 \mathrm{E}+06$ & & 25.57 & 25.82 & 25.80 & & 0.07 & 0.08 & 0.07 & & 11.47 & 11.69 & 11.69 \\
\hline 28 & 776 & $2.10 E+07$ & $2.46 \mathrm{E}+07$ & $2.36 E+07$ & & 25.66 & 25.86 & 25.71 & & 0.29 & 0.32 & 0.30 & & 11.56 & 11.73 & 11.60 \\
\hline 29 & 778 & $2.11 E+07$ & $2.28 \mathrm{E}+07$ & $2.09 E+07$ & & 26.36 & 26.46 & 26.42 & & 0.29 & 0.30 & 0.26 & & 12.26 & 12.33 & 12.31 \\
\hline 30 & 786 & $9.69 E+05$ & $1.06 \mathrm{E}+06$ & $1.16 \mathrm{E}+06$ & & 25.04 & 25.45 & 25.60 & & 0.01 & 0.01 & 0.01 & & 10.94 & 11.32 & 11.49 \\
\hline 31 & 788 & $3.14 E+06$ & $3.97 \mathrm{E}+06$ & $3.79 \mathrm{E}+06$ & & 24.45 & 24.54 & 24.61 & & 0.04 & 0.05 & 0.05 & & 10.35 & 10.41 & 10.50 \\
\hline 32 & 790 & $4.52 \mathrm{E}+07$ & $5.08 \mathrm{E}+07$ & 4.67E+07 & & 25.08 & 25.24 & 25.17 & & 0.62 & 0.66 & 0.59 & & 10.98 & 11.11 & 11.06 \\
\hline 33 & 792 & $2.44 E+08$ & $2.76 \mathrm{E}+08$ & $2.59 E+08$ & & 25.78 & 25.96 & 25.92 & & 3.34 & 3.60 & 3.26 & & 11.68 & 11.83 & 11.81 \\
\hline 34 & 810 & 4.24E+06 & 4.67E+06 & $4.85 E+06$ & & 24.00 & 24.12 & 24.22 & & 0.06 & 0.06 & 0.06 & & 9.90 & 9.99 & 10.11 \\
\hline 35 & 812 & $4.32 \mathrm{E}+06$ & $5.10 \mathrm{E}+06$ & $4.90 E+06$ & & 24.35 & 24.57 & 24.57 & & 0.06 & 0.07 & 0.06 & & 10.25 & 10.44 & 10.46 \\
\hline 36 & 814 & $2.52 E+06$ & $3.13 E+06$ & $2.70 E+06$ & & 24.76 & 24.89 & 24.85 & & 0.03 & 0.04 & 0.03 & & 10.66 & 10.76 & 10.74 \\
\hline 37 & 814 & $2.28 \mathrm{E}+06$ & $3.52 \mathrm{E}+06$ & $3.09 \mathrm{E}+06$ & & 26.30 & 26.26 & 26.27 & & 0.03 & 0.05 & 0.04 & & 12.20 & 12.13 & 12.16 \\
\hline 38 & 816 & $2.44 E+06$ & $2.39 \mathrm{E}+06$ & $2.44 E+06$ & & 25.19 & 25.37 & 25.32 & & 0.03 & 0.03 & 0.03 & & 11.09 & 11.24 & 11.21 \\
\hline 39 & 818 & $1.03 E+07$ & 1.17E+07 & $1.14 \mathrm{E}+07$ & & 25.87 & 25.94 & 25.99 & & 0.14 & 0.15 & 0.14 & & 11.77 & 11.81 & 11.88 \\
\hline 40 & 820 & $9.49 \mathrm{E}+06$ & $1.03 E+07$ & $8.71 E+06$ & & 26.35 & 26.39 & 26.32 & & 0.13 & 0.13 & 0.11 & & 12.25 & 12.26 & 12.21 \\
\hline 41 & 836 & 4.33E+08 & $4.82 E+08$ & $4.82 E+08$ & & 24.51 & 24.64 & 24.64 & & 5.93 & 6.27 & 6.06 & & 10.41 & 10.51 & 10.53 \\
\hline 42 & 864 & $4.70 E+06$ & $4.55 \mathrm{E}+06$ & $4.62 E+06$ & & 25.08 & 25.35 & 25.24 & & 0.06 & 0.06 & 0.06 & & 10.98 & 11.22 & 11.13 \\
\hline 43 & 866 & $2.08 \mathrm{E}+06$ & $2.69 \mathrm{E}+06$ & $2.06 E+06$ & & 25.32 & 25.50 & 25.52 & & 0.03 & 0.03 & 0.03 & & 11.22 & 11.37 & 11.41 \\
\hline
\end{tabular}

Figure 6:11. Areas and retention times as well as standardized areas and retention times of all the PE species found in goldfish retina membranes. 


\begin{tabular}{|c|c|c|c|c|c|c|c|c|c|}
\hline$\Delta$ & A & $\mathrm{R}$ & $S$ & $\mathrm{~T}$ & U & v & W & $\mathrm{X}$ & $\mathrm{Y}$ \\
\hline 1 & $M / Z$ & \multicolumn{4}{|c|}{ Area } & & \multicolumn{3}{|c|}{ Retention Time } \\
\hline 2 & & & & & & & & & \\
\hline 3 & & Raw Ave. & Standardized Ave. & Stand. Dev. & \% Stand. Dev. & & Standardized Ave. & Stand. Dev. & \% Stand. Dev. \\
\hline 4 & 454 & & & & & & & & \\
\hline 5 & & & & & & & & & \\
\hline 6 & & & & & & & & & \\
\hline 7 & 674 & $1.50 E+06$ & 0.02 & 0.00 & 24.58 & & 10.84 & 0.08 & 0.73 \\
\hline 8 & 688 & $1.72 \mathrm{E}+06$ & 0.02 & 0.00 & 21.49 & & 9.68 & 0.13 & 1.39 \\
\hline 9 & 690 & $4.40 E+06$ & 0.06 & 0.00 & 8.07 & & 10.19 & 0.03 & 0.34 \\
\hline 10 & 702 & $5.34 \mathrm{E}+06$ & 0.07 & 0.01 & 15.86 & & 11.44 & 0.11 & 0.97 \\
\hline 11 & 704 & $2.08 \mathrm{E}+06$ & 0.03 & 0.00 & 10.25 & & 11.61 & 0.15 & 1.25 \\
\hline 12 & 716 & $6.86 \mathrm{E}+06$ & 0.09 & 0.01 & 12.79 & & 10.55 & 0.07 & 0.67 \\
\hline 13 & 718 & $1.67 \mathrm{E}+07$ & 0.22 & 0.02 & 7.65 & & 11.18 & 0.09 & 0.78 \\
\hline 14 & 728 & $3.12 E+06$ & 0.04 & 0.00 & 5.35 & & 11.97 & 0.06 & 0.51 \\
\hline 15 & 730 & $4.16 \mathrm{E}+06$ & 0.05 & 0.00 & 8.74 & & 12.11 & 0.15 & 1.23 \\
\hline 16 & 736 & $1.32 \mathrm{E}+06$ & 0.02 & 0.00 & 5.73 & & 9.83 & 0.07 & 0.67 \\
\hline 17 & 738 & $3.11 \mathrm{E}+06$ & 0.04 & 0.01 & 22.04 & & 10.24 & 0.11 & 1.08 \\
\hline 18 & 740 & $5.06 \mathrm{E}+06$ & 0.07 & 0.00 & 0.65 & & 10.80 & 0.10 & 0.94 \\
\hline 19 & 744 & $9.94 E+06$ & 0.13 & 0.01 & 7.20 & & 11.43 & 0.08 & 0.72 \\
\hline 20 & 746 & $1.77 \mathrm{E}+07$ & 0.23 & 0.02 & 10.56 & & 12.10 & 0.08 & 0.66 \\
\hline 21 & 750 & $1.29 \mathrm{E}+07$ & 0.17 & 0.00 & 2.81 & & 11.34 & 0.08 & 0.66 \\
\hline 22 & 762 & $2.89 \mathrm{E}+06$ & 0.04 & 0.01 & 17.19 & & 10.04 & 0.11 & 1.13 \\
\hline 23 & 764 & $6.63 E+07$ & 0.87 & 0.04 & 4.79 & & 10.83 & 0.04 & 0.38 \\
\hline 24 & 766 & $2.06 \mathrm{E}+07$ & 0.27 & 0.03 & 10.04 & & 11.14 & 0.04 & 0.36 \\
\hline 25 & 768 & $2.89 E+07$ & 0.38 & 0.02 & 6.13 & & 11.77 & 0.06 & 0.53 \\
\hline 26 & 772 & $2.89 E+06$ & 0.04 & 0.01 & 19.74 & & 12.18 & 0.14 & 1.18 \\
\hline 27 & 774 & $5.68 \mathrm{E}+06$ & 0.07 & 0.01 & 11.48 & & 11.62 & 0.13 & 1.09 \\
\hline 28 & 776 & $2.31 \mathrm{E}+07$ & 0.30 & 0.02 & 5.61 & & 11.63 & 0.09 & 0.76 \\
\hline 29 & 778 & $2.16 \mathrm{E}+07$ & 0.28 & 0.02 & 6.29 & & 12.30 & 0.04 & 0.29 \\
\hline 30 & 786 & $1.06 \mathrm{E}+06$ & 0.01 & 0.00 & 4.68 & & 11.25 & 0.28 & 2.50 \\
\hline 31 & 788 & $3.63 E+06$ & 0.05 & 0.00 & 9.20 & & 10.42 & 0.08 & 0.72 \\
\hline 32 & 790 & $4.76 \mathrm{E}+07$ & 0.62 & 0.04 & 5.93 & & 11.05 & 0.07 & 0.59 \\
\hline 33 & 792 & $2.60 E+08$ & 3.40 & 0.18 & 5.20 & & 11.77 & 0.08 & 0.69 \\
\hline 34 & 810 & $4.59 \mathrm{E}+06$ & 0.06 & 0.00 & 2.73 & & 10.00 & 0.11 & 1.05 \\
\hline 35 & 812 & $4.77 \mathrm{E}+06$ & 0.06 & 0.00 & 5.94 & & 10.38 & 0.12 & 1.12 \\
\hline 36 & 814 & $2.78 \mathrm{E}+06$ & 0.04 & 0.00 & 10.34 & & 10.72 & 0.05 & 0.49 \\
\hline 37 & 814 & $2.96 \mathrm{E}+06$ & 0.04 & 0.01 & 18.84 & & 12.16 & 0.04 & 0.29 \\
\hline 38 & 816 & $2.42 \mathrm{E}+06$ & 0.03 & 0.00 & 4.56 & & 11.18 & 0.08 & 0.71 \\
\hline 39 & 818 & $1.11 \mathrm{E}+07$ & 0.15 & 0.01 & 4.23 & & 11.82 & 0.06 & 0.47 \\
\hline 40 & 820 & $9.49 E+06$ & 0.12 & 0.01 & 10.42 & & 12.24 & 0.03 & 0.22 \\
\hline 41 & 836 & $4.65 E+08$ & 6.09 & 0.17 & 2.85 & & 10.48 & 0.06 & 0.61 \\
\hline 42 & 864 & $4.62 E+06$ & 0.06 & 0.00 & 5.41 & & 11.11 & 0.12 & 1.09 \\
\hline 43 & 866 & $2.28 \mathrm{E}+06$ & 0.03 & 0.00 & 15.71 & & 11.33 & 0.10 & 0.88 \\
\hline
\end{tabular}

Figure 6:12. Averages of standardized areas and retention times of all the PE species found in goldfish retina membranes, also standard deviation values and \% standard deviation values are shown. 


\begin{tabular}{|c|c|c|c|c|c|c|c|c|c|c|c|c|c|}
\hline$\Delta$ & A & AA & $\mathrm{AB}$ & $\mathrm{AC}$ & $A D$ & $\mathrm{AE}$ & $\mathrm{AF}$ & AG & $\mathrm{AH}$ & $\mathrm{Al}$ & $\mathrm{AJ}$ & AK & $\mathrm{AL}$ \\
\hline 1 & $\mathrm{M} / \mathrm{Z}$ & \multicolumn{4}{|c|}{ Standardized July Area } & & \multicolumn{4}{|c|}{ Standardized January Area } & & & \\
\hline 2 & & & & & & & & & & & & Fold Change & $T$-test \\
\hline 3 & & Trial 1 & Trial 2 & Trial 3 & Ave. & & Trial 1 & Trial 2 & Trial 3 & Ave. & & & \\
\hline 4 & 454 & & & & & & & & & & & & \\
\hline \multicolumn{14}{|l|}{5} \\
\hline \multicolumn{14}{|l|}{6} \\
\hline 7 & 674 & 0.02 & 0.02 & 0.01 & 0.02 & & 0.02 & 0.01 & 0.02 & 0.02 & & 1.11 & 0.703 \\
\hline 8 & 688 & 0.02 & 0.03 & 0.02 & 0.02 & & 0.04 & 0.03 & 0.04 & 0.04 & & 0.56 & 0.02 \\
\hline 9 & 690 & 0.05 & 0.06 & 0.06 & 0.06 & & 0.10 & 0.06 & 0.08 & 0.08 & & 0.71 & 0.078 \\
\hline 10 & 702 & 0.08 & 0.06 & 0.07 & 0.07 & & 0.15 & 0.08 & 0.11 & 0.11 & & 0.62 & 0.13 \\
\hline 11 & 704 & 0.03 & 0.03 & 0.02 & 0.03 & & 0.07 & 0.05 & 0.06 & 0.06 & & 0.45 & 0.012 \\
\hline 12 & 716 & 0.08 & 0.09 & 0.10 & 0.09 & & 0.17 & 0.09 & 0.13 & 0.13 & & 0.68 & 0.184 \\
\hline 13 & 718 & 0.22 & 0.20 & 0.24 & 0.22 & & 0.38 & 0.23 & 0.29 & 0.30 & & 0.73 & 0.159 \\
\hline 14 & 728 & 0.04 & 0.04 & 0.04 & 0.04 & & 0.04 & 0.02 & 0.04 & 0.03 & & 1.19 & 0.287 \\
\hline 15 & 730 & 0.06 & 0.05 & 0.05 & 0.05 & & 0.13 & 0.09 & 0.10 & 0.10 & & 0.52 & 0.014 \\
\hline 16 & 736 & 0.02 & 0.02 & 0.02 & 0.02 & & 0.02 & 0.02 & 0.02 & 0.02 & & 0.89 & 0.371 \\
\hline 17 & 738 & 0.04 & 0.05 & 0.03 & 0.04 & & 0.13 & 0.08 & 0.11 & 0.11 & & 0.38 & ( $\quad 0.01$ \\
\hline 18 & 740 & 0.07 & 0.07 & 0.07 & 0.07 & & 0.08 & 0.06 & 0.07 & 0.07 & & 0.96 & 0.707 \\
\hline 19 & 744 & 0.13 & 0.14 & 0.12 & 0.13 & & 0.25 & 0.13 & 0.18 & 0.19 & & 0.69 & 0.159 \\
\hline 20 & 746 & 0.25 & 0.24 & 0.21 & 0.23 & & 0.34 & 0.22 & 0.25 & 0.27 & & 0.85 & 0.374 \\
\hline 21 & 750 & 0.17 & 0.16 & 0.17 & 0.17 & & 0.21 & 0.12 & 0.15 & 0.16 & & 1.06 & 0.746 \\
\hline 22 & 762 & 0.03 & 0.04 & 0.04 & 0.04 & & 0.06 & 0.04 & 0.05 & 0.05 & & 0.74 & 0.149 \\
\hline 23 & 764 & 0.85 & 0.91 & 0.84 & 0.87 & & 1.26 & 0.70 & 0.93 & 0.96 & & 0.90 & 0.591 \\
\hline 24 & 766 & 0.25 & 0.30 & 0.26 & 0.27 & & 0.59 & 0.34 & 0.45 & 0.46 & & 0.59 & 0.061 \\
\hline 25 & 768 & 0.37 & 0.40 & 0.36 & 0.38 & & 0.53 & 0.32 & 0.38 & 0.41 & & 0.92 & 0.643 \\
\hline 26 & 772 & 0.04 & 0.05 & 0.03 & 0.04 & & 0.08 & 0.04 & 0.06 & 0.06 & & 0.63 & 0.12 \\
\hline 27 & 774 & 0.07 & 0.08 & 0.07 & 0.07 & & 0.10 & 0.06 & 0.08 & 0.08 & & 0.91 & 0.571 \\
\hline 28 & 776 & 0.29 & 0.32 & 0.30 & 0.30 & & 0.37 & 0.23 & 0.30 & 0.30 & & 1.01 & 0.968 \\
\hline 29 & 778 & 0.29 & 0.30 & 0.26 & 0.28 & & 0.33 & 0.20 & 0.30 & 0.28 & & 1.02 & 0.891 \\
\hline 30 & 786 & 0.01 & 0.01 & 0.01 & 0.01 & & 0.03 & 0.02 & 0.02 & 0.03 & & 0.54 & 0.07 \\
\hline 31 & 788 & 0.04 & 0.05 & 0.05 & 0.05 & & 0.05 & 0.03 & 0.03 & 0.04 & & 1.18 & 0.323 \\
\hline 32 & 790 & 0.62 & 0.66 & 0.59 & 0.62 & & 0.86 & 0.53 & 0.68 & 0.69 & & 0.90 & 0.526 \\
\hline 33 & 792 & 3.34 & 3.60 & 3.26 & 3.40 & & 4.38 & 2.67 & 3.48 & 3.51 & & 0.97 & 0.836 \\
\hline 34 & 810 & 0.06 & 0.06 & 0.06 & 0.06 & & 0.13 & 0.08 & 0.10 & 0.10 & & 0.58 & 0.03 \\
\hline 35 & 812 & 0.06 & 0.07 & 0.06 & 0.06 & & 0.09 & 0.06 & 0.07 & 0.07 & & 0.88 & 0.429 \\
\hline 36 & 814 & 0.03 & 0.04 & 0.03 & 0.04 & & 0.05 & 0.03 & 0.04 & 0.04 & & 0.92 & 0.624 \\
\hline 37 & 814 & 0.03 & 0.05 & 0.04 & 0.04 & & 0.06 & 0.04 & 0.06 & 0.05 & & 0.73 & 0.164 \\
\hline 38 & 816 & 0.03 & 0.03 & 0.03 & 0.03 & & 0.05 & 0.03 & 0.04 & 0.04 & & 0.87 & 0.437 \\
\hline 39 & 818 & 0.14 & 0.15 & 0.14 & 0.15 & & 0.24 & 0.15 & 0.17 & 0.19 & & 0.76 & 0.174 \\
\hline 40 & 820 & 0.13 & 0.13 & 0.11 & 0.12 & & 0.29 & 0.20 & 0.22 & 0.24 & & 0.53 & 0.02 \\
\hline 41 & 836 & 5.93 & 6.27 & 6.06 & 6.09 & & 8.65 & 4.72 & 6.42 & 6.60 & & 0.92 & 0.677 \\
\hline 42 & 864 & 0.06 & 0.06 & 0.06 & 0.06 & & 0.10 & 0.06 & 0.07 & 0.08 & & 0.79 & 0.293 \\
\hline 43 & 866 & 0.03 & 0.03 & 0.03 & 0.03 & & 0.05 & 0.03 & 0.03 & 0.03 & & 0.87 & 0.535 \\
\hline \multicolumn{14}{|l|}{44} \\
\hline 45 & & & & & & & & & & & & red & 31 \\
\hline 46 & & & & & & & & & & & & green & 6 \\
\hline 47 & & & & & & & & & & & & decreased in July & 6 \\
\hline
\end{tabular}

Figure 6:13. Fold changes and $p$-values at $\alpha=0.05$ between January and July of all the PE species found in goldfish retina membranes. 
method rejects them (this is called a type II error) ${ }^{12}$. The FDR approach was properly described first by Benjamini and Hochberg in $1995^{13}$ and has been identified as one of the most cited statistical methods by Ryan and Woodall, $2005^{14}$. There is also a great deal of scientific literature that uses Benjamini-Hochberg method for FDR correction in lipidomics and genomics work ${ }^{15-19}$. The description of other FDR procedures (not as commonly used as $\mathrm{BH}$ method) is outside of the scope of this thesis.

The $\mathrm{BH}$ method is quite simple to incorporate into the calculations. Once there are p-values obtained from a t-test; those values can be arranged from smallest to largest, and they are ranked as the smallest $p$-value being 1 and the largest $p$-value having the highest rank value in the dataset. The example for this is shown in Figure 6:14 columns $\mathrm{G}$ and $\mathrm{H}$. The individual $\mathrm{p}$-values are compared to $\mathrm{BH}$ critical values that are calculated as $(i / m) Q^{20}$, where $i$ is the rank, $m$ is the total number of tests (in this work the total number of certain type of phospholipids found above LOQ), and $Q$ is the chosen false discovery rate (often called $\alpha$ ). Then of interest is to find the largest $p$-value that has smaller corresponding $\mathrm{BH}$ critical value $(\mathrm{p}<(i / m) Q)$ (values in column $\mathrm{J}$ are calculated: $\left.J_{i}=G_{i}-l_{i}\right)$; then that $p$-value as well all the smaller $p$-values will be significant. This is shown in Figure 6:14 columns I and J. Figure 6:14 shows $\mathrm{BH}$ method on goldfish eye retina membrane PEs; it can be observed that from 6 significant PEs found from t-test 5 remained significant after $\mathrm{BH}$ correction. $\mathrm{BH}$ correction was done per phospholipid type versus all phospholipids in the sample; this is because 4 phospholipid classes have their specific LOQ and LOD values. 
High-resolution data: As mentioned in the previous chapters as well as earlier in this chapter, even though, low-resolution data gives a great way to distinguish between phospholipid types and nominal masses, there is still an issue of not knowing the exact identity of the phospholipids due to their isomeric and isobaric nature. However, it would be of great interest to know the exact identity of the phospholipids that are downregulated or upregulated in cellular membranes in adjustment to cooler temperatures or in adjustment to different seasons. High-resolution data can narrow down the possibilities in this process by providing more accurate data. The Agilent 6550 iFunnel Q-TOF was used for high-resolution data collection; the MS instrument was calibrated to $0.01 \mathrm{Da}$ and MS data was collected in positive ion mode. Agilent Mass Hunter Qualitative Analysis B.07.00 software was used for data extraction.

The exact theoretical masses of significantly changing phospholipids between the seasons were extracted from Lipidomics Gateway lipid database. These values were inputted into Mass Hunter software with error $\pm 0.01 \mathrm{Da}$ and compared to values obtained from MS runs. Figure 6:15 shows the internal standard (13:0 Lyso PC) peak; the mass from the spectrum is $454.2885 \mathrm{~m} / \mathrm{z}$, the mass from the database is $454.2829 \mathrm{~m} / \mathrm{z}$. It can be observed that internal standard mass difference between two sources is 0.0056 Th. This mass shift is useful in phospholipid identification; as masses of phospholipids are expected to shift by approximately the same amount and in the same direction for everything for the same MS run. If a particular phospholipid mass on the MS spectrum is a bit lower compared to the database value for the same run, it is most likely because it is not a peak of the phospholipid that is expected. 


\begin{tabular}{|c|c|c|c|c|c|}
\hline $\mathrm{F}$ & G & $\mathrm{H}$ & 1 & $J$ & K \\
\hline \multicolumn{2}{|c|}{ PE } & & $\mathrm{Q}=0.15$ & \multicolumn{2}{|c|}{ FIVE SIGNIFICANT PEAKS } \\
\hline \multirow[t]{2}{*}{$M / Z$} & $p$ values & $\operatorname{rank}(i)$ & $(\mathrm{i} / \mathrm{m})^{*} \mathrm{Q}$ & & \\
\hline & & & BH value & & \\
\hline 738 & 0.01049 & 1 & 0.00405 & 0.00644 & \\
\hline 704 & 0.01201 & 2 & 0.00811 & 0.00390 & \\
\hline 730 & 0.01449 & 3 & 0.01216 & 0.00232 & \\
\hline 820 & 0.01974 & 4 & 0.01622 & 0.00352 & \\
\hline 688 & 0.01986 & 5 & 0.02027 & -0.00041 & \\
\hline 810 & 0.02959 & 6 & 0.02432 & 0.00527 & \\
\hline 766 & 0.06107 & 7 & 0.02838 & 0.03269 & \\
\hline 786 & 0.06954 & 8 & 0.03243 & 0.03710 & \\
\hline 690 & 0.07785 & 9 & 0.03649 & 0.04136 & \\
\hline 772 & 0.11972 & 10 & 0.04054 & 0.07918 & \\
\hline 702 & 0.12992 & 11 & 0.04459 & 0.08533 & \\
\hline 762 & 0.14905 & 12 & 0.04865 & 0.10040 & \\
\hline 718 & 0.15881 & 13 & 0.05270 & 0.10611 & \\
\hline 744 & 0.15916 & 14 & 0.05676 & 0.10241 & \\
\hline 814 & 0.16421 & 15 & 0.06081 & 0.10340 & \\
\hline 818 & 0.17406 & 16 & 0.06486 & 0.10920 & \\
\hline 716 & 0.18412 & 17 & 0.06892 & 0.11520 & \\
\hline 728 & 0.28742 & 18 & 0.07297 & 0.21444 & \\
\hline 864 & 0.29253 & 19 & 0.07703 & 0.21551 & \\
\hline 788 & 0.32281 & 20 & 0.08108 & 0.24173 & \\
\hline 736 & 0.37123 & 21 & 0.08514 & 0.28610 & \\
\hline 746 & 0.37376 & 22 & 0.08919 & 0.28457 & \\
\hline 812 & 0.42901 & 23 & 0.09324 & 0.33577 & \\
\hline 816 & 0.43666 & 24 & 0.09730 & 0.33937 & \\
\hline 790 & 0.52647 & 25 & 0.10135 & 0.42512 & \\
\hline 866 & 0.53511 & 26 & 0.10541 & 0.42971 & \\
\hline 774 & 0.57143 & 27 & 0.10946 & 0.46197 & \\
\hline 764 & 0.59065 & 28 & 0.11351 & 0.47713 & \\
\hline 814 & 0.62371 & 29 & 0.11757 & 0.50614 & \\
\hline 768 & 0.64251 & 30 & 0.12162 & 0.52089 & \\
\hline 836 & 0.67684 & 31 & 0.12568 & 0.55116 & \\
\hline 674 & 0.70309 & 32 & 0.12973 & 0.57336 & \\
\hline 740 & 0.70679 & 33 & 0.13378 & 0.57301 & \\
\hline 750 & 0.74618 & 34 & 0.13784 & 0.60834 & \\
\hline 792 & 0.83598 & 35 & 0.14189 & 0.69409 & \\
\hline 778 & 0.89053 & 36 & 0.14595 & 0.74458 & \\
\hline 776 & 0.96777 & 37 & 0.15000 & 0.81777 & \\
\hline
\end{tabular}

Figure 6:14. Benjamini-Hochberg $(B H)$ correction of goldfish retina membranes significant PEs at $\alpha=0.15$ $(\alpha=Q)$. 


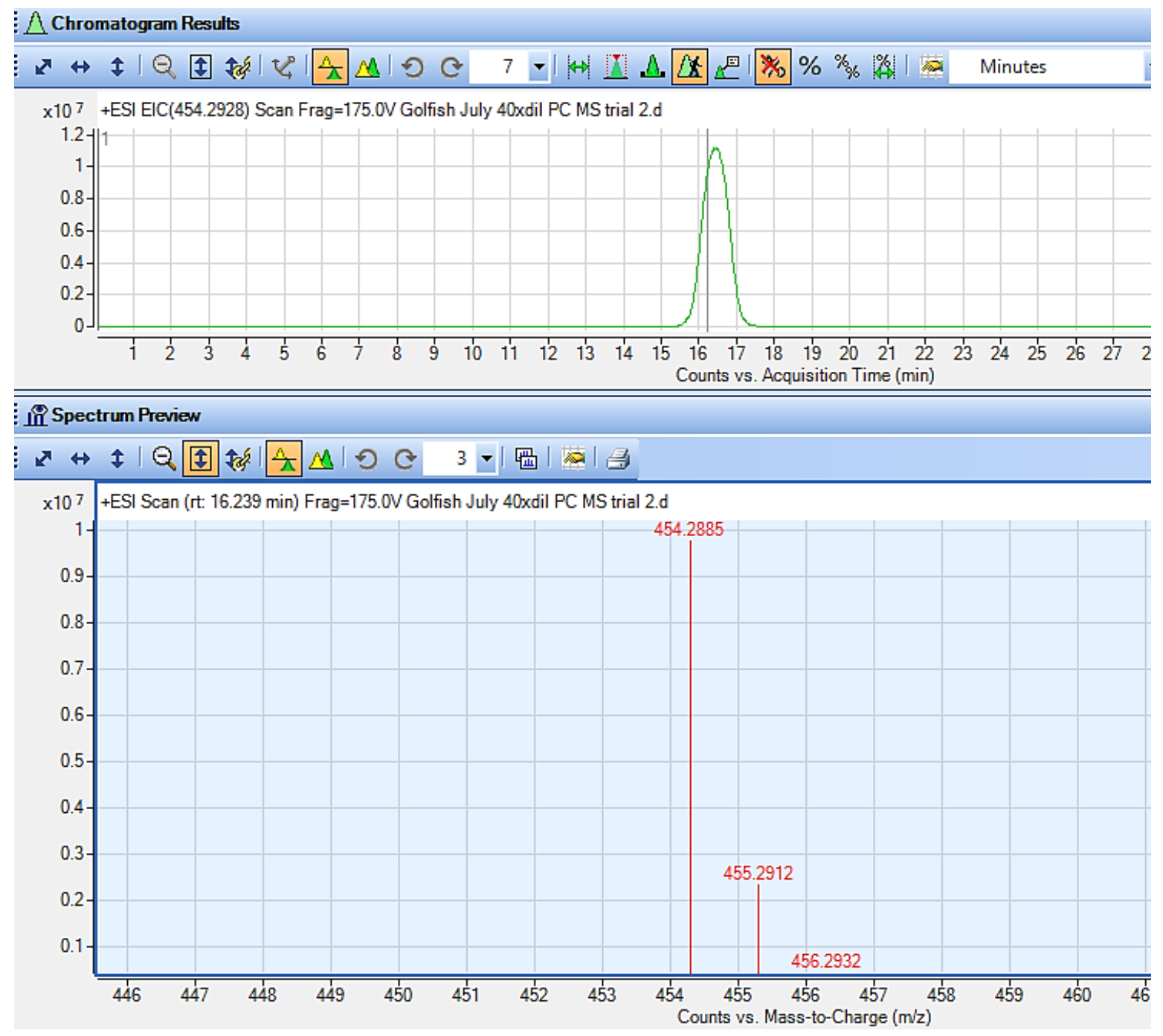

Figure 6:15. Internal standard peak from high-resolution data.

High-resolution MS data gives enough resolution to differentiate if specific phospholipids are diacyl species (both fatty acid groups are liked to glycerol via ester group) or ethereal-acyl/plasmalogen-acyl species or if there is high saturation on the phospholipid in question. For every nominal mass for the same type of phospholipid class 
there can be, and often are, three exact masses of species mathematically possible. For example, for PE of 704 Th, the exact theoretical masses are 704.5225 Th for a diacyl species, 704.5589 Th for an ethereal-acyl/plasmalogen-acyl species and 704.4291 Th for a highly saturated diacyl species. It has been observed, however, that in biological samples quite often all of these species are present in various proportions to each other, highly saturated species are generally observed to the smaller extent. On the MS spectrum, the peak for 704.4291 Th was mass shifting in the opposite direction of the internal standard peak, and thus it was concluded that there is no PE with 704.4291 Th present in the sample, so no highly saturated diacyl species for 704 Th in the sample. The peak for 704.5589 Th was shifting in the right direction with the internal standard peak. However, it was shifting by a lot higher amount compared to other peaks on the same spectrum. It was concluded that it is highly likely that PE with 704.5589 Th is not present in the sample and that mostly nominal mass of 704 Th is 704.5225 Th as it was shifting in the right direction and it the right amount compared to internal standard. The nominal mass of 704 Th was identified as mostly diacyl species.

Fatty acid identification: To obtain more structural information about the significant phospholipids, MS/MS experiments were carried out in the negative ion mode. This allows phospholipids to lose their fatty acids chains as negative ion fragments and permit their identification. This data elucidates the length of the carbon chains are and the number of double bonds in the lipid; however, it does not indicate were those double bonds are, their configuration (cis or trans), nor indicate the regiochemistry of the acyl chains. 
Figures 6:16 and 6:17 show one example of how fatty acid chain information was extracted. Figure 6:16 shows an MS/MS spectrum for peak 686.4756 Th (negative ion mode), which has been identified as a diacyl species from MS data alone. The product ion spectrum reveals only one peak with an exact mass of $253.2208 \mathrm{~m} / \mathrm{z}$, indicating a 16:1 (16 carbons with one double bond, $\mathrm{CH}_{3} \mathrm{C}_{14} \mathrm{H}_{26} \mathrm{COO}^{-}$) fatty acid chain. The whole list of MS/MS peaks for 686.4756 Th is shown in Figure 6:17, which shows $100 \%$ abundance for 253.2208 Th peak. Other peaks in Figure 6:17 are shown as relative abundances to peak at 253.2208 Th. It can be concluded that a diacyl species with an exact mass of 686.4756 Th in negative ion mode has two $16: 1$ fatty acid chains on its $s n-1$ and sn-2 locations.

The Lipidomics Gateway lipid database consists of lipids that have been curated as well as of lipids that have been computationally generated. For 688.4766 Th in positive ion mode, the database shows three curated possibilities with both fatty acid chains as

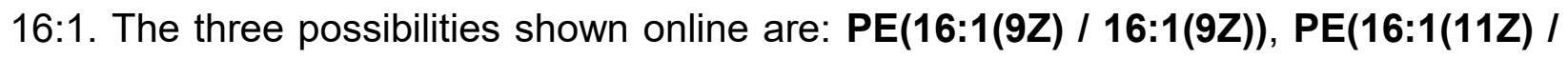
16:1(11Z)) and PE(16:1(5Z)/ 16:1(5Z)). It appears that when the fatty acid chains in sn1 and sn-2 locations are the same, they also have their double bonds in the same location and with the same configuration. However, with the experimental techniques used in this research work, it is not possible to determine the exact location and configuration of the double bonds of fatty acid chains in the complex sample of lipids and the above database observation cannot be confirmed at this time. Also, it is quite possible that there are multiple structural options for actual $16: 1$ fatty acid chain in one complex biological lipidomic sample. 


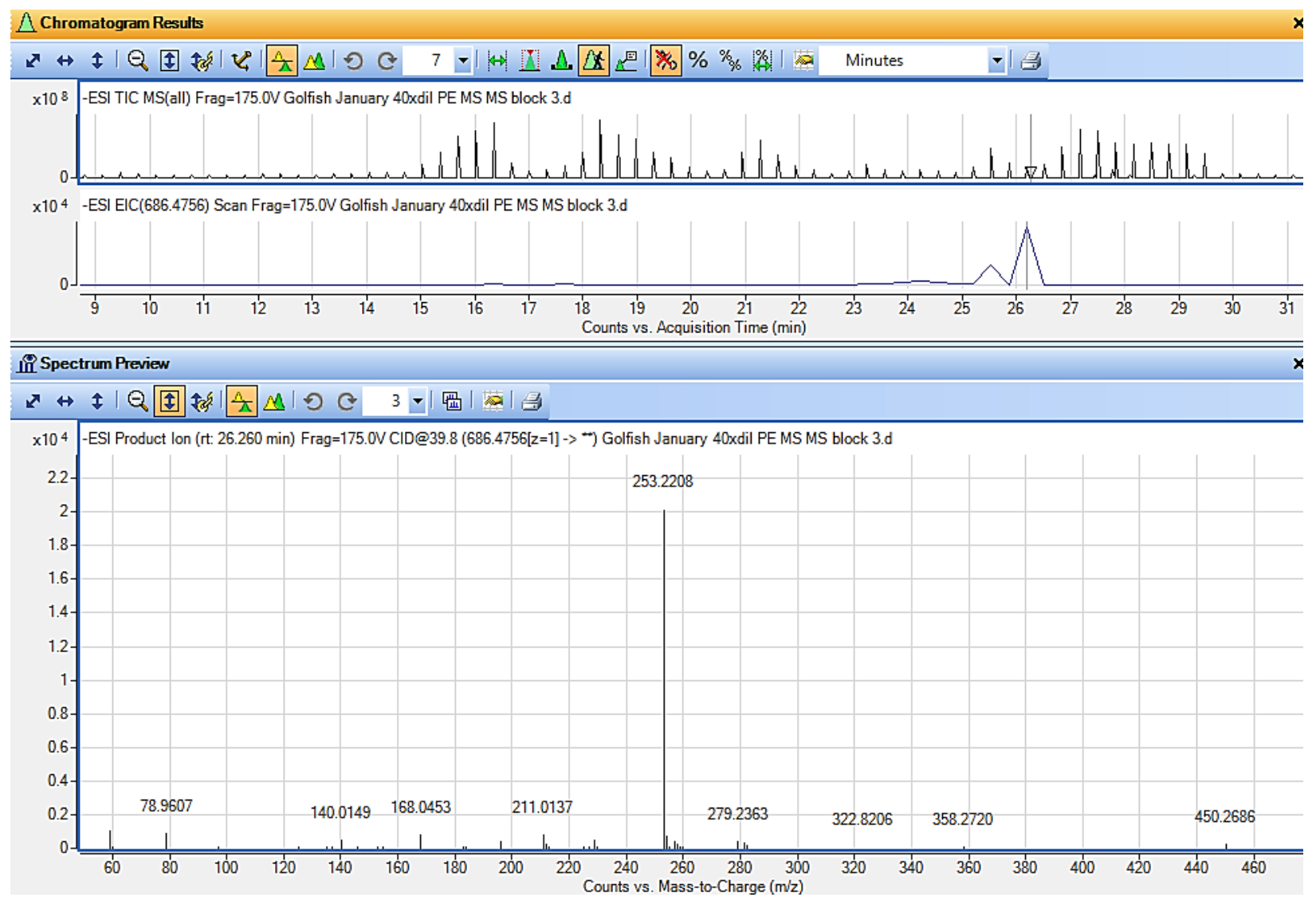

Figure 6:16. Spectrum preview shows MS/MS spectrum for ion 686.4756 Th.

Ether versus plasmalogen: Vinyl-ether (plasmalogen) and alkyl-ether (ethereal) fatty acids chains do not fragment in MS/MS experiments and need other methods of identification and differentiation as they have same exact mass. In work by Fhaner et al., 2012 they describe formic acid hydrolysis reaction that hydrolyzes all the plasmalogens ${ }^{21}$. The data from Fhaner et al., 2012 demonstrated that $80 \%$ formic acid (FA) could be used to selectively and completely hydrolyze vinyl-ether containing lipids, without degradation of diacyl or alkyl-ether containing species ${ }^{21}$. The procedure was followed similarly on lipidomic samples from squirrel liver and goldfish eye retina. The high-resolution MS was collected before (unmodified) and after FA treatment ( $80 \%$ FA) and results for the two 
significant PEs that could be either ethereal-acyl or plasmalogen-acyl species are shown in Figure 6:18.

\begin{tabular}{|c|c|c|c|c|c|c|}
\hline 林MS Peaks & s One: - Pr & duct Ion ( & $\mathrm{t}: 26.260 \mathrm{mi}$ & $(68$ & 6.4756 & $5 \rightarrow-7$ \\
\hline $\mathrm{m} / \mathrm{z}$ & Species $\not$ & Abund & Abund $\% \multimap$ & $Z+\square$ & Sat & $\mathrm{m} / \mathrm{z}$ \\
\hline | 217.1235 & & 17.52 & 0.09 & & & \\
\hline 225.1884 & & 95.12 & 0.47 & & & \\
\hline 227.2053 & & 97.9 & 0.49 & & & \\
\hline 229.0228 & & 443.44 & 2.2 & 1 & & \\
\hline 229.6711 & & 10.92 & 0.05 & 2 & & \\
\hline 230.0279 & & 89.2 & 0.44 & 1 & & \\
\hline 231.0214 & & 29.22 & 0.15 & 1 & & \\
\hline 239.2427 & & 34.22 & 0.17 & & & \\
\hline 251.2075 & & 38.84 & 0.19 & & & \\
\hline 253.0906 & & 16.53 & 0.08 & & & \\
\hline 253.1248 & & 22.56 & 0.11 & & & \\
\hline 253.1615 & & 41.17 & 0.2 & 2 & & \\
\hline 253.2208 & & 20140.57 & 100 & 1 & & \\
\hline 253.358 & & 13.01 & 0.06 & 2 & & \\
\hline 253.3855 & & 11.01 & 0.05 & 2 & & \\
\hline 253.4296 & & 23.17 & 0.12 & 2 & & \\
\hline 253.4901 & & 29.03 & 0.14 & 2 & & \\
\hline 253.5341 & & 39.04 & 0.19 & 2 & & \\
\hline 253.5517 & & 23.22 & 0.12 & 2 & & \\
\hline 253.6112 & & 23.01 & 0.11 & 2 & & \\
\hline 253.6512 & & 33.56 & 0.17 & 2 & & \\
\hline 253.6664 & & 14.21 & 0.07 & 2 & & \\
\hline 253.6883 & & 12.16 & 0.06 & 2 & & \\
\hline 253.718 & & 11.18 & 0.06 & 2 & & \\
\hline 253.7441 & & 7.06 & 0.04 & 2 & & \\
\hline 253.8819 & & 19.41 & 0.1 & & & \\
\hline 253.941 & & 26.46 & 0.13 & & & \\
\hline 253.9637 & & 10.01 & 0.05 & & & \\
\hline
\end{tabular}

Figure 6:17. The whole peak list extracted for MS-MS spectrum for ion 686.4756 Th. 


\begin{tabular}{|c|c|c|c|c|c|c|c|c|c|c|c|}
\hline$\Delta$ & A & B & C & D & $\mathrm{E}$ & $\mathrm{F}$ & G & $\mathrm{H}$ & 1 & J & $\mathrm{K}$ \\
\hline 1 & & & & 128.18 & 1.281757 & & & & & & \\
\hline \multicolumn{12}{|l|}{2} \\
\hline 3 & & & \multicolumn{4}{|c|}{ Area } & & & & & \\
\hline 4 & & & 1 & 2 & 3 & Ave & $\%$ remaining after deriv & & standardized FA areas & & $\%$ ether remaining \\
\hline 5 & 688.519 & unmod & $2.54 E+05$ & $6.56 E+04$ & $3.20 \mathrm{E}+04$ & 1.17E+05 & $\begin{array}{r}100.00 \\
\end{array}$ & & & & \\
\hline 6 & & $80 \% \mathrm{FA}$ & $6.55 \mathrm{E}+03$ & $2.76 \mathrm{E}+03$ & $1.75 \mathrm{E}+03$ & $3.69 \mathrm{E}+03$ & 3.14 & & $2.88 \mathrm{E}+03$ & & 2.45 \\
\hline \multicolumn{12}{|l|}{7} \\
\hline 8 & 730.5649 & unmod & $2.24 \mathrm{E}+06$ & 2.37E+06 & $2.60 \mathrm{E}+06$ & $2.40 E+06$ & 100.00 & & & & \\
\hline 9 & & $80 \%$ FA & $1.04 \mathrm{E}+06$ & $9.38 \mathrm{E}+05$ & $5.28 \mathrm{E}+05$ & $8.34 \mathrm{E}+05$ & 34.68 & & $6.51 \mathrm{E}+05$ & & 27.05 \\
\hline \multicolumn{12}{|l|}{10} \\
\hline \multicolumn{12}{|l|}{11} \\
\hline 12 & \multicolumn{3}{|c|}{ Theoretical } & $\operatorname{Exp}$ & & \multicolumn{2}{|c|}{ Shift in FA samples } & & & & \\
\hline 13 & & $\mathrm{~m} / \mathrm{z}$ & & $\mathrm{m} / \mathrm{z}$ & & & & & & & \\
\hline \multicolumn{12}{|l|}{14} \\
\hline 15 & & 454.2928 & & 454.2873 & & 0.0055 & & & & & \\
\hline \multicolumn{12}{|l|}{16} \\
\hline 17 & & 688.5276 & & 688.519 & & 0.0086 & & & & & \\
\hline 18 & & 704.5589 & & 704.5654 & & -0.0065 & & & & & \\
\hline 19 & & 730.5745 & & 730.5649 & & 0.0096 & & & & & \\
\hline \multicolumn{12}{|l|}{20} \\
\hline 21 & & & & & & & & & & & \\
\hline
\end{tabular}

Figure 6:18. Excel sheet is showing proportions of ethereal-acyl and plasmalogen-acyl species for two PE peaks.

As mentioned above, the peak for PE with 704.5589 Th is not present in the sample and thus is not considered for vinyl-ether or alkyl-ether species differentiation. The nominal mass of 704 Th was identified as mostly diacyl lipid due to it mass shift that is in the opposite direction compared to rest of the peaks for that run. The only peaks that needed differentiation between vinyl-ether or alkyl-ether species were 688.5190 Th and 730.5649 Th PEs in goldfish retina samples. Data has shown that most of the time peaks are a combination of vinyl-ether or alkyl-ether species and not $100 \%$ of one specific lipid. Figure 6:18 compares area values of vinyl-ether/alkyl-ether peaks before and after FA treatment, and relative percentage of alkyl-ether species remaining is calculated as vinylether species are entirely hydrolyzed and do no show on the spectra. Vinyl-ether/alkylether peaks are standardized toward diacyl peaks (Figure 6:18 column I) to avoid the assumption that other peaks are not affected by FA treatment to any extent.10 random diacyl peaks were chosen to see variation in area values before and after FA treatment; 
the total variation value is highlighted red in Figure 6:18 cell D1 and used to standardize vinyl-ether/alkyl-ether peaks. Also, phospolipid peak masses were matched before and after formic acid treatment. It has been found that the two significant PEs were mostly vinyl-ether species: the peak at $\mathrm{m} / \mathrm{z} 688.5190$ is $98 \%$ vinyl-ether PE and the peak at $\mathrm{m} / \mathrm{z}$ 730.5649 is $73 \%$ vinyl-ether PE.

\subsection{Sources of error}

The main limitation in lipidomics work done in this thesis is the lack of biological replicates; to account for this limitation and errors associated with it pooled samples were used for analysis. The poled sample means that one sample was a combination of tissues of at least two animals; in case on liver and leg muscle samples, the needed mass was obtained from couple of different animals, in case of eye retina samples, three random retinas were combined to make one sample for lipidomics studies.

Another important source of error comes from phospholipid area values extraction using MultiQuant software. This software does not integrate peaks that are close to LOQ or does a poor job on it. Consequently, many peaks had to be manually calibrated. Even though, care was taken to be as accurate as possible in the manual integration, sometimes there was no clear cut of where peaks start or end, and estimation was used.

There is also a possibility of tissues and thus phospholipids degradation that could come from storage of those tissues prior to analysis. In case of squirrel and frog, the tissues available for studies were in the freezer for close to 10 years prior to lipid extraction. In Chapter 4 from the reference it was mentioned that storage at $-80^{\circ} \mathrm{C}$ up to 
10 years does not significantly influence serum phospholipid quality. However, some degradation is always a possibility. Phospholipids were analyzed within a week (usually within half a week from extraction) avoiding lipid oxidation after lipid extraction. Extracted lipids were also stored in $-20^{\circ} \mathrm{C}$ freezer under nitrogen within this analysis time period.

\subsection{Conclusion}

This chapter showed the whole workflow of a lipidomic data set, which was quite lengthy and tedious due to a large amount of information and complexity of the data. The individual results for different animals and their tissues have been shown in their respective chapters earlier. The purpose of this chapter was to demonstrate the lipidomics data processing that was performed.

AB Sciex QTRAP 4000 MS instrument is able to do precursor ion scan, and neutral loss scan experiments that permit the identification of different phospholipid classes and data from this instrument is sufficient for phospholipid quantification. The limitation of this instrument is that it is low-resolution and identification of exact masses is limited to a first decimal place. Biological samples are complex; it was seen that there are peaks at every single unit in the MS region of $700-900 \mathrm{~m} / \mathrm{z}$ for phospholipid species. Also, due to isomeric and isobaric nature of these molecules, there are many structural possibilities for one nominal mass. The Agilent iFunnel 6550 Q-TOF MS instrument is used for highresolution experiments and has been shown to be sufficient in the differentiation of phospholipids with the same nominal mass. 
Future work could be suggested to narrow down possible phospholipid structures to their unique identities. However, this would require determination of the location and configuration of the double bonds in the fatty acid chains as well as the determination of fatty acid chain location ( $s n-1$ and $s n-2$ position) in the glycerol backbone of the phospholipids. This kind of work is possible on the individual phospholipids, however, complex mixtures of lipids that contain many combinations of various lipids present quite a challenge for this task.

\subsection{References}

(1) Murray, K. K.; Boyd, R. K.; Eberlin, M. N.; Langley, G. J.; Li, L.; Naito, Y. Pure Appl. Chem. 2013, 85 (7), 1515-1609.

(2) Armbruster, D. A.; Pry, T. Clin. Biochem. Rev. 2008, 29 Suppl 1, S49-52.

(3) Canez, C. R.; Shields, S. W. J.; Bugno, M.; Wasslen, K. V.; Weinert, H. P.; Willmore, W. G.; Manthorpe, J. M.; Smith, J. C. Anal. Chem. 2016, 88 (14), 69967004.

(4) Ivanova, P. T.; Cerda, B. A.; Horn, D. M.; Cohen, J. S.; McLafferty, F. W.; Brown, H. A. Proc. Natl. Acad. Sci. U. S. A. 2001, 98 (13), 7152-7157.

(5) Busik, J. V; Reid, G. E.; Lydic, T. A. Methods Mol. Biol. 2009, 579, 33-70.

(6) Löhmann, C.; Schachmann, E.; Dandekar, T.; Villmann, C.; Becker, C.-M. J. Neurochem. 2010, 114 (4), 1119-1134.

(7) Sciex, M. AB Appl. Biosyst. 2005, 3200 Q TRA (Manual), 134.

(8) Moyer, T. O.; Gambler, E.; Lynch-Davis, K.; Driskell, S. Source Math. Teach. 2008, 102 (2), 151-153.

(9) Pandis, N. Am. J. Orthod. Dentofac. Orthop. 2015, 148 (2), 350-351.

(10) Milton, J. S. Statistical methods in the biological and health sciences, Third Ed.; McGraw-Hill, 1999.

(11) Benjamini, Y. J. R. Stat. Soc. Ser. B 2010, 72 (4), 405-416.

(12) Perneger, T. V. BMJ 1998, 316 (7139), 1236-1238. 
(13) Benjamini, Y.; Hochberg, Y. Journal of the Royal Statistical Society. Series B (Methodological). WileyRoyal Statistical Society 1995, pp 289-300.

(14) Ryan, T. P.; Woodall, W. H. J. Appl. Stat. 2005, 32 (5), 461-474.

(15) Grace, M. S.; Dempsey, P. C.; Sethi, P.; Mundra, P. A.; Mellett, N. A.; Weir, J. M.; Owen, N.; Dunstan, D. W.; Meikle, P. J.; Kingwell, B. A. J. Clin. Endocrinol. Metab. 2017, 102 (6), 1991-1999.

(16) Edlow, A. G.; Vora, N. L.; Hui, L.; Wick, H. C.; Cowan, J. M.; Bianchi, D. W.; Sanchez-Margalet, V. PLoS One 2014, 9 (2).

(17) Liu, L.; Zhou, X.; Zhang, Y.; Liu, Y.; Yang, L.; Pu, J.; Zhu, D.; Zhou, C.; Xie, P. Behav. Brain Res. 2016, 305, 148-156.

(18) Samouda, H.; De Beaufort, C.; Stranges, S.; Hirsch, M.; Van Nieuwenhuyse, J.P.; Dooms, G.; Gilson, G.; Keunen, O.; Leite, S.; Vaillant, M.; Lair, M.-L.; Dadoun, F. Pediatr. Diabetes 2016, 17 (4), 300-308.

(19) Husse, J.; Kiehn, J.-T.; Barclay, J. L.; Naujokat, N.; Meyer-Kovac, J.; Lehnert, H.; Oster, H. Sleep 2017, 40 (6).

(20) McDonald, J. H. Multiple comparisons - Handbook of Biological Statistics, 3rd ed.; Sparky House Publishing: Baltimore, MD, 2014.

(21) Fhaner, C. J.; Liu, S.; Ji, H.; Simpson, R. J.; Reid, G. E. Anal. Chem. 2012, 84 (21), 8917-8926. 


\section{Chapter 7. The development of methods to simplify MS spectra for better identification of peptides}

\subsection{Abstract}

Protein dynamics play an essential role in many cellular processes. MS is a useful tool to investigate protein dynamics; however low abundance proteins are challenging to detect or quantify. Improvements in MS-based technologies have helped researchers understand protein dynamics better and monitor biochemical processes more closely and precisely. This proteomics chapter focuses on a technique that has been developed to improve MS signal for biological molecules (here discussed in the context of peptides). This technique is called trimethylation enhancement using diazomethane (TrEnDi) which allows the creation of fixed permanent positive charges on the peptide molecules. Diazomethane has been produced by different procedures, two of which will be described herein each having their advantages and disadvantages. Despite some optimization issues involving TrEnDi on peptides, this technique has been shown to be successful with phosphorylated and methylated peptides. The primary results show a consistent loss of a neutral mass of 126 Da during fragmentation of phosphorylated peptides; this is a loss of dimethyl hydrogen phosphate molecule. TrEnDi also forms unique charge states on the phosphorylated and methylated peptides which makes them easier to observe on the MS. These results open a new direction for monitoring phosphorylated and methylated peptides in medical field. 


\subsection{Diazomethane and methylation of commercial peptides}

\subsubsection{Diazomethane}

Diazomethane is a strong methylating agent that is also shock sensitive, explosive and highly toxic. It is also carcinogenic molecule and should be handled in a fume hood. Diazomethane is not commercially available as it is hazardous to be shipped and hence it is made in-house. Safety precautions are paramount when working with it. Diazomethane was produced mostly in small quantities (on average about $10 \mathrm{~mL}$ ethereal diazomethane solutions with concentrations ranging $0.1-0.3 \mathrm{M}$ ) to prevent long storage times. It was always made behind a blast shield in the fume food and dissolved into an ethereal solvent, as recommended in the relevant Sigma-Aldrich Technical Bulletin ${ }^{1}$.

At room temperature diazomethane is a yellow gas; it has a boiling point of $-23^{\circ} \mathrm{C}$ and a melting point of $-145^{\circ} \mathrm{C}$. Crystal formation of diazomethane should be avoided as sharp edges on the chemical are prone for explosion ${ }^{1}$. Diazomethane has different precursors from which it can be synthesized. The two precursors are going to be described here: $N$-methyl- $N$-nitroso-urea (NMU) and $N$-methyl- $N$-nitroso- $p$-toluenesulfonamide (Diazald).

\subsubsection{Synthesis via N-Methyl-N-nitroso-urea (NMU)}

Synthesis of diazomethane from NMU is shown in Figure 7:1 (last reaction). NMU was prepared by established protocols, stored dry in the freezer and used as needed for diazomethane. Initially, diazomethane was prepared in the following way: $5 \mathrm{~g}$ of $\mathrm{KOH}$ was dissolved in $5 \mathrm{~mL}$ MilliQ water ( $50 \% \mathrm{KOH}$ solution) and allowed to cool, then $5 \mathrm{~mL}$ of $\mathrm{KOH}$ 
solution was combined with $10 \mathrm{~mL}$ cooled ether solution in $25 \mathrm{~mL}$ Erlenmeyer flask and placed in an ice bath, added was $0.5 \mathrm{~g}$ of NMU. The mixture allowed to react for 5 minutes shaking it occasionally, then yellow diazomethane layer (bottom layer) was transferred using a round flamed tip into an empty Erlenmeyer flask, it was stoppered and wrapped with parafilm and placed into a $-20^{\circ} \mathrm{C}$ freezer.

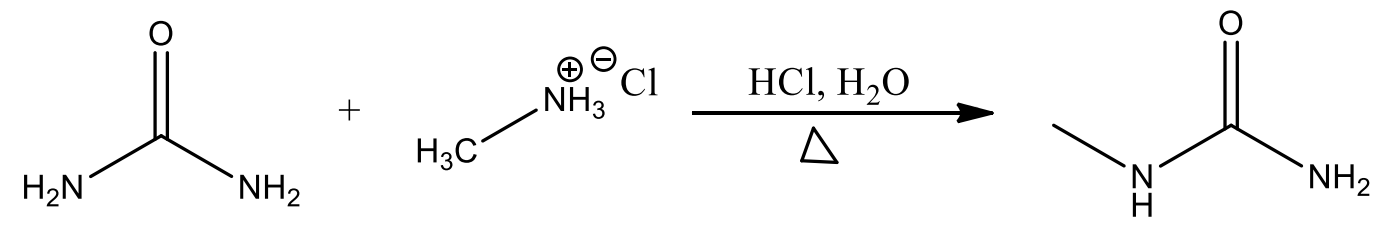<smiles>CNC(N)=O</smiles>

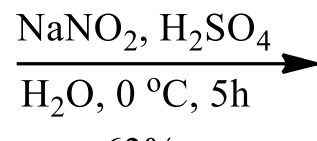
$63 \%$

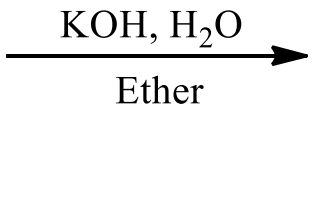<smiles>CN(N=O)C(N)=O</smiles>

NMU<smiles>CN(N=O)C(N)=O</smiles><smiles>[CH2-][N+]#N</smiles><smiles>C=C</smiles><smiles>C=[N+]=[N-]</smiles>

Figure 7:1. Synthesis of diazomethane via NMU.

7.2.1.2 Synthesis via and N-Methyl-N-nitroso-p-toluenesulfonamide (Diazald)

Synthesis of diazomethane was also accomplished via Diazald. The procedure for this is in detail described in Sigma-Aldrich technical bulletin ${ }^{1}$ and is summarized in 
Figure $7: 2$. This procedure includes heating the reaction mixture to $65^{\circ} \mathrm{C}$ where diazomethane is formed as a gas and then collected into the ether. Pearce, 1980 tried hydrolysing Diazald with $30 \% \mathrm{KOH}$ (in house Diazald was hydrolysed with $30 \% \mathrm{KOH}$ as well) at $0^{\circ}$ to $-5^{\circ} \mathrm{C}$ and there was no diazomethane formed. They also mention that reaction requires a temperature of at least $55^{\circ} \mathrm{C}$ or higher for diazomethane to form ${ }^{2}$.

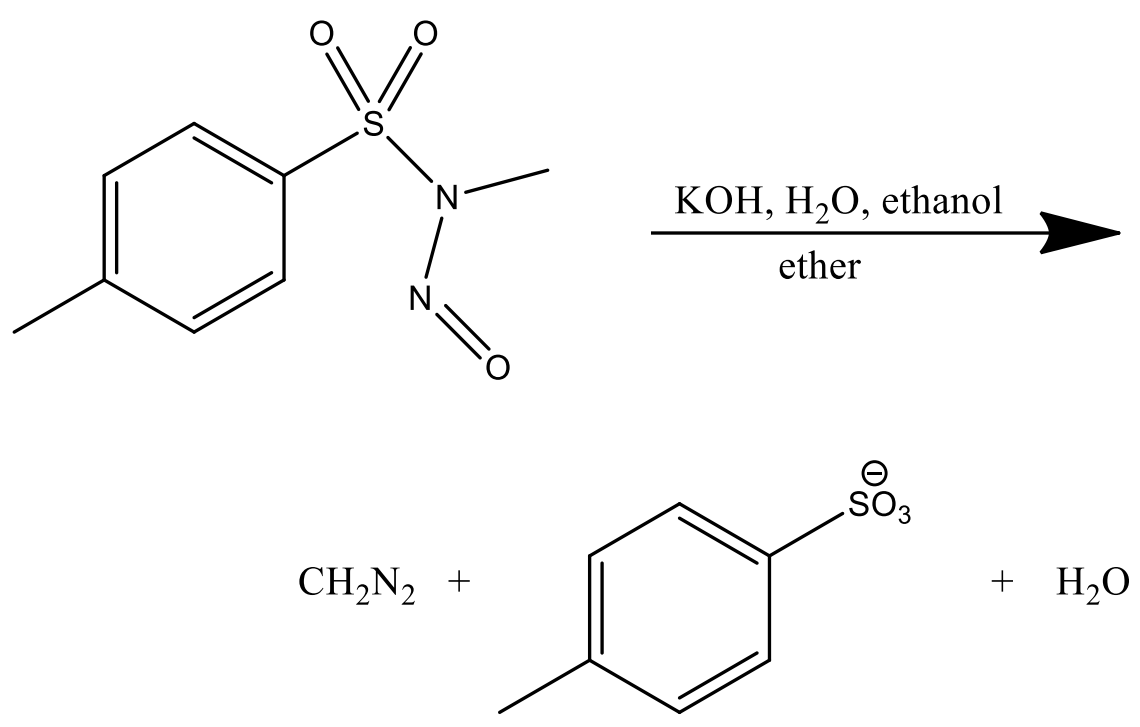

Figure 7:2. Synthesis of diazomethane via Diazald.

\subsubsection{Diazomethane titrations}

Preparation via NMU was a preferred method for diazomethane preparation since it was faster, it did not require heating of the reaction vessel, and the diazomethane formed immediately partitioned into the ether. However, after many methylating procedures of various peptides using NMU diazomethane, it was observed that peptides 
were consistently undermethylated. It was of interest to determine the concentration of diazomethane produced, so that production of diazomethane could be optimized to be the same every time and that peptide methylation would be more consistent. Two methods of titration of diazomethane were explored: one with glacial acidic acid (GAA) in toluene and another one with 4-fluorobenzoic acid in deuterated acetone.

The reaction of an acid with diazomethane would produce a corresponding methyl ester for that acid and diazomethane would be neutralized. GAA was also used for quenching any unused diazomethane. The titration with GAA was done with a diluted GAA solution $(0.87 \mathrm{M})$, and the endpoint of titration was determined visually; the characteristic yellow color of diazomethane would have disappeared from diazomethane solution. This kind of endpoint determination is a subject to a human error, and precise concentration is difficult to conclude.

Rendina and Kingsbury, 2012 showed the protocol that they developed for titrating non-stabilized diazoalkane solutions. They used 2- fluorobenzoic acid to over titrate their species and then compared residual acid, and the corresponding formed ester peaks via ${ }^{19} \mathrm{~F}$ nuclear magnetic resonance (NMR) spectrometry; by quantifying two peaks and ultimately determining diazoalkane concentrations ${ }^{3}$. This protocol was adapted for using 4-fluorobenzoic acid (due to availability) to titrate diazomethane solutions.

The comparative results are shown for GAA and 4-fluorobenzoic acid titrations of diazomethane solutions in Table 7:1. Diazomethane was prepared via two methods both with NMU precursor: once with $50 \% \mathrm{KOH}$ solution and another time with $45 \% \mathrm{KOH}$ solution. Rendina and Kingsbury, 2012 studied the concentrations of diazomethane produced when either $30 \%, 40 \%$ or $50 \% \mathrm{KOH}$ solution were used with NMU. Their results 
showed that the highest and the quickest amounts of diazomethane were produced with $30 \% \mathrm{KOH}$ solution, followed by $40 \% \mathrm{KOH}$ solution and then only $50 \% \mathrm{KOH}$ solution. They obtained $90 \%$ yield of diazomethane after a couple of minutes on NMU with the $30 \% \mathrm{KOH}$ solution reaction. Comparatively, 5 minutes of $\mathrm{NMU}$ reaction with $50 \% \mathrm{KOH}$ solution would yield under $10 \%$ diazomethane and required approximately 3 hours to produce a $70 \%$ yield. The curve for $30 \%$ and $40 \% \mathrm{KOH}$ solutions with NMU are shown to be very steep by Rendina and Kingsbury, 2012; that is why $45 \% \mathrm{KOH}$ solution was chosen for comparative purposes of diazomethane production.

Table 7:1. Diazomethane concentrations when it is produced from NMU precursor and either 50\% or $45 \%$ $\mathrm{KOH}$ solutions. Reaction time was 7 minutes in each case. Concentration values via two titration methods are compared.

\begin{tabular}{|c|c|c|c|}
\hline Trial & $\begin{array}{c}\text { KOH solution } \\
\text { used to make } \\
\text { diazomethane } \\
\text { with NMU (\%) }\end{array}$ & $\begin{array}{c}\text { Diazomethane concentration } \\
\text { via GAA in toluene (M) }\end{array}$ & $\begin{array}{c}\text { Diazomethane } \\
\text { concentration via 4- } \\
\text { fluorobenzoic acid in d- } \\
\text { acetone (M) }\end{array}$ \\
\cline { 1 - 2 } 1 & \multirow{3}{*}{50} & 0.205 & 0.104 \\
\hline 2 & & 0.139 & 0.109 \\
\cline { 1 - 1 } 3 & \multirow{2}{*}{45} & 0.143 & 0.102 \\
\hline 1 & & 0.256 & 0.168 \\
\cline { 3 - 4 } 2 & & 0.177 & 0.120 \\
\hline 3 & & 0.209 & 0.165 \\
\hline
\end{tabular}

The results in Table 7:2 show that more diazomethane was produced with $45 \%$ $\mathrm{KOH}$ solution than $50 \% \mathrm{KOH}$ solution in reaction with $\mathrm{NMU}$. This result was consistent with the supporting information from the reference. Also, it can be observed that there is a smaller variance in concentration values that were determined via 4-fluorobenzoic acid titration rather that GAA acid titration. This result was also expected, as GAA relies mostly on the visual determination. Relying on the 4-fluorobenzoic acid method for diazomethane 
concentration determination, it was then of interest to compare methods of diazomethane production via NMU or Diazald precursors.

Table 7:2. Diazomethane concentration values. The reaction of NMU with $50 \% \mathrm{KOH}$ was carried out during a 20-minute period, small amounts of sample were taken at specific time points and analyzed for diazomethane concentration; two separate batches were made at two different dates. Diazald was also used to make diazomethane on two different dates, however, in these cases, three trials of each batch were sampled and analyzed for concentration.

\begin{tabular}{|c|c|c|}
\hline $\begin{array}{c}\text { Time of diazomethane } \\
\text { reaction (min.) }\end{array}$ & $\begin{array}{c}\text { Diazomethane } \\
\text { concentrations (M) - batch } \\
1\end{array}$ & $\begin{array}{c}\text { Diazomethane } \\
\text { concentrations (M) - batch } \\
2\end{array}$ \\
\hline 5 & 0.038 & 0.035 \\
\hline 10 & 0.096 & 0.063 \\
\hline 13 & 0.108 & 0.073 \\
\hline 16 & 0.116 & 0.073 \\
\hline 20 & 0.089 & 0.084 \\
\hline \multicolumn{3}{|c|}{ Diazomethane from Diazald } \\
\hline Avg. conc. of 3 trials & 0.301 & 0.323 \\
\hline
\end{tabular}

Table 7:2 summarizes concentration values of diazomethane obtained via two precursors. It can be observed that the current method via Diazald produced more concentrated diazomethane and there seem to be fewer variations from batch to batch. The reaction of NMU with $45 \% \mathrm{KOH}$ was also carried out and sampled for specific time points (data not shown). However, it still produced diazomethane at lower concentrations (avg. $0.130 \mathrm{M}$ at $9 \mathrm{~min}$.) than the Diazald reaction. The peptide methylation reactions were done with diazomethane produced via both methods. However, even with diazomethane via Diazald method, undermethylated peaks were observed. Undermethylation was persistent and unfortunately not consistent and hindered continuing investigation on TrEnDi modified peptides, though some promising results were seen and shown next. 


\subsubsection{HPLC separation of TrEnDi modified peptides}

Trimethylation Enhancement using Diazomethane (TrEnDi) modified peptides were fragmented to yield increased sensitivity via MS. Previous results in this area have successfully demonstrated the enhancement in peptide analyses. TrEnDi creates fixed, permanent positive charges on peptide analytes making identification of these species easier on MS. Wasslen et al., 2014 demonstrated TrEnDi on tryptically digested bovine serum albumin (BSA) and quantified the peptide AEFVEVTK via LC-MS which was not detected `before modification ${ }^{4}$. It has been observed that diazomethane trimethylates the $\mathrm{N}$-terminus of peptides as well as all functional groups with pKa values below 11. Table 7:1 summarizes all the amino acids with side chain pKa values below 11 and shows their methylated structures after TrEnDi modification.

\subsubsection{Peptide methylation procedure}

The chemistry of methylating peptides was conducted via the use of microfluidics. A fused silica column with an inner diameter of $200 \mu \mathrm{m}$ was fritted (described in methodology sections in lipidomics chapters) and packed with Poly-SULFOETHYL A (strong cation exchange (SCX)) chromatographic resin to approximately $10 \mathrm{~cm}$. This was done on an in-house constructed reaction vessel that is pressured by nitrogen gas (Figure 7:3). This packed column was preconditioned with $10 \mathrm{mM}$ potassium phosphate buffer at $\mathrm{pH} 3$ and peptides of interest were loaded onto the column in $50 \mathrm{mM} \mathrm{H}_{3} \mathrm{PO}_{4}$ solution (1:10 of peptides: $50 \mathrm{mM} \mathrm{H}_{3} \mathrm{PO}_{4}$ ). About $10 \mu \mathrm{g}$ of peptides were loaded on the $10 \mathrm{~cm} \mathrm{SCX}$ column. Then, an ethereal diazomethane solution was run through the column to methylate the 
peptides of interest. The peptide loading and methylation procedures are shown in Figure $7: 4$.

Table 7:3. Shown are pKa values for amino acid chains (obtained from chapter 2 amino acid table) and TrEnDi modification of these amino acids. This table is the modified version from Wasslen et al., 2014 (Supporting Information) 4 . The rest of the amino acid chains are not shown as all of their side chain pKa values are above $12^{4}$.

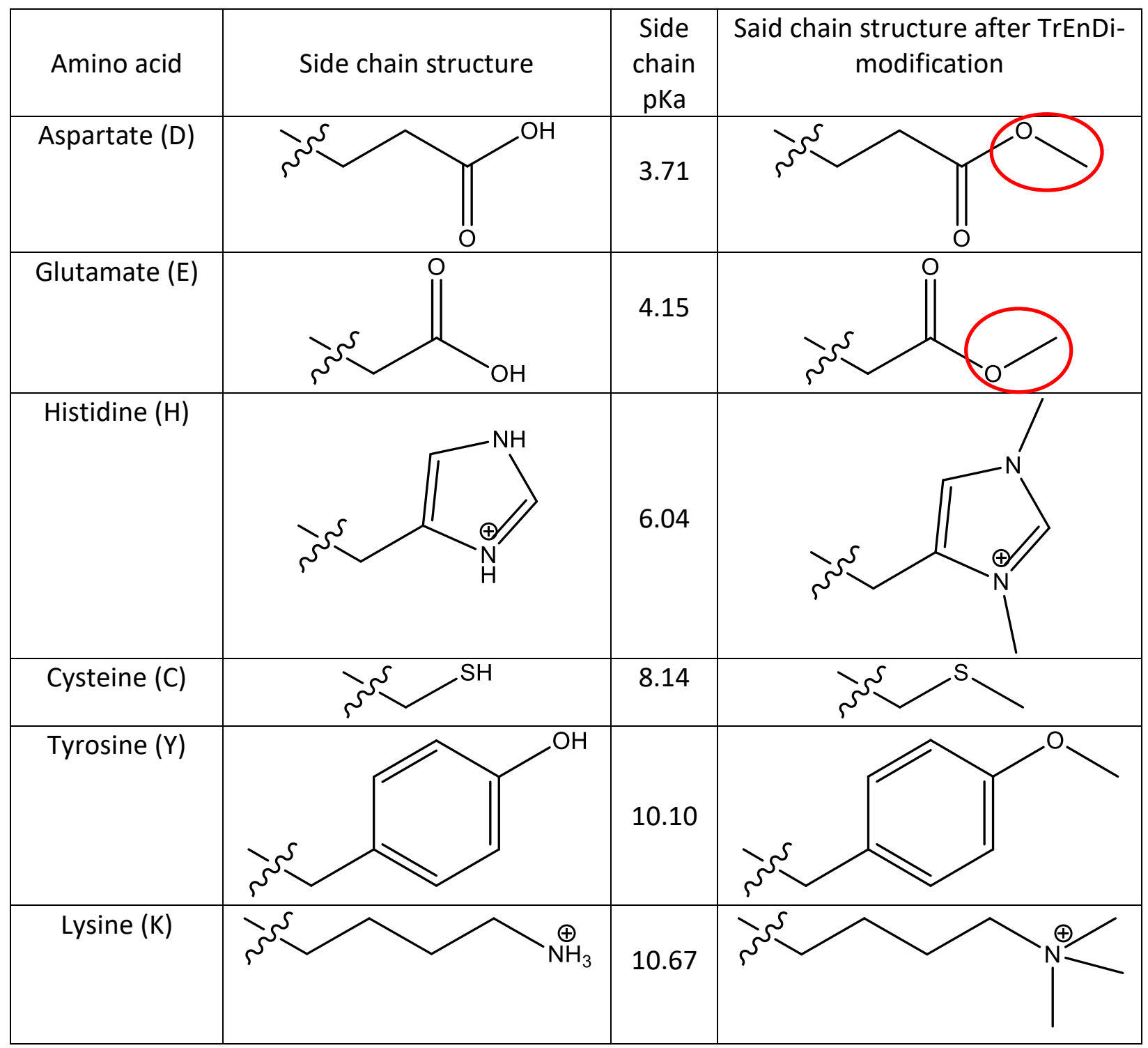



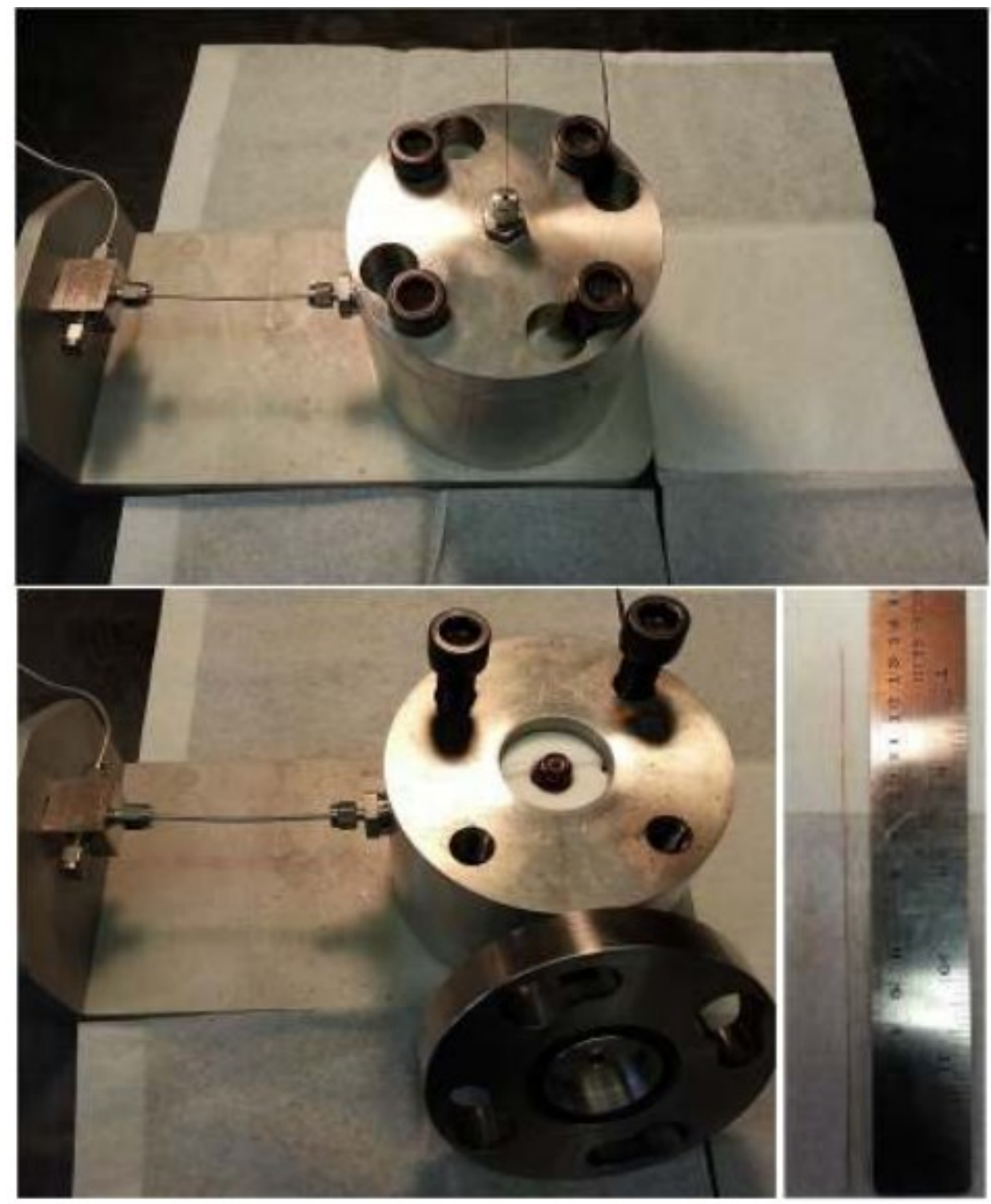

Figure 7:3. High pressure vessel used for column chemistry. Image above shows the vessel set up with column, image below shows solvent compartment where vials are inserted. 


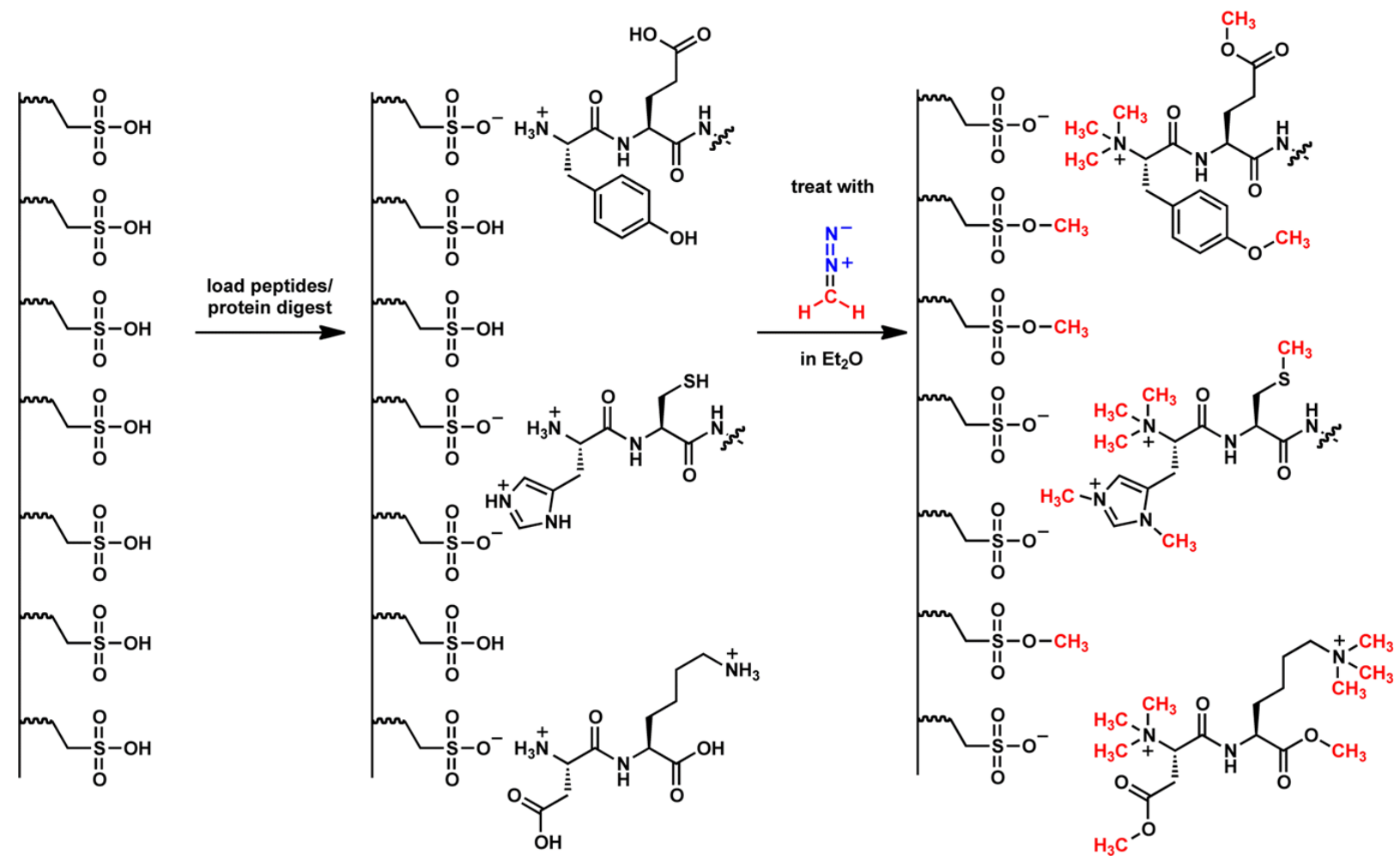

Figure 7:4. Loading peptides on an SCX column in the first step. Methylating peptides on the SCX column by running ethereal diazomethane through the peptide loaded column in the second step.

\subsubsection{Elution of peptides from SCX column and separation of TrEnDi modified peptides on HPLC}

Following modification, parameters to chromatographically collect and elute the TrEnDi modified peptides were tested. One variable was elution with ammonium bicarbonate. This worked well and fully modified peptides were observed in the MS spectra. However, over time hydrolysis and aminolysis of methyl ester groups (the ones circled in Table 7:3 and C-terminus methyl ester) were observed and MS spectra had various amounts of methyl groups for the same peptides. The goal was to create methylated peptides with a consistent number of methyl groups to simplify the spectra. Elution with a $\mathrm{NaOH}$ solution was attempted to hydrolyze the methyl esters during 
collection instead of allowing them to hydrolyze over time. This produced stably methylated peptides. However, an issue with $\mathrm{NaOH}$ elution was that a desalting step was required before MS analysis as sodium is a non-volatile salt that interferes with ESI-MS. Even following desalting, sodiated adduct peaks were observed in the spectra. The sodiated peaks might not be an issue when there are only small amounts of peptides to be recognized in the spectra, however, with long proteins and numerous peptides present in it, sodiated peaks make MS spectra quite complicated.

For direct spray experiments about 2-4 $\mu \mathrm{L}$ of peptide sample was used with a Proxeon nanoelectrospray emitter (Thermo Scientific, Odense, Denmark) followed by direct analysis of peptides using an Q-TOF MS instrument equipped with a nanoelectrospray ionization source (AB Sciex, Framingham, MA, USA). Spectra were obtained using a nanoelectrospray voltage of $2000 \mathrm{~V}$, declustering and focusing potentials at 60 and $120 \mathrm{~V}$, respectively, and an $\mathrm{MS}^{2}$ collision energy for $\mathrm{MS}^{2}$ experiments ranged between 33 and $35 \mathrm{eV} .4$

To eliminate sodiation, triethylammonium acetate (TEAA) buffer was used to elute TrEnDi modified peptides from the column. TEAA is a volatile salt eluent which is expected to produce a stable derivatized product with no-desalting requirement. The structure of TEAA is shown in Figure 7:5. 


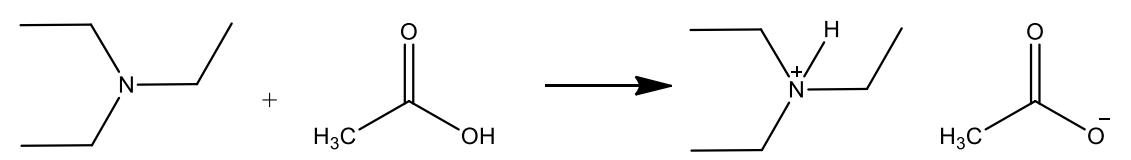

Figure 7:5. TEAA salt is prepared from equimolar quantities of triethylamine and acetic acid.

Another important point to mention is that following derivatization, SCX columns lose their binding ability as ethereal diazomethane solution passes through it methylating sulfonic groups on the SCX resin. To test the binding ability of a TrEnDi modified SCX column, an attempt was made to load peptides onto a modified column. As suspected, peptides did not bind onto the TrEnDi modified SCX column as the load solution flowthrough yielded peptide signals on MS. Interestingly, TrEnDi modified peptides have been observed to elute from a diazomethane treated column by passing $\mathrm{H}_{2} \mathrm{O}$ through it (TEAA elutes peptides as well). Elution with water is possible because SCX resin gets methylated and methylated peptides do not bind to the separating resin any longer. It is possible that some peptides do get lost during methylation process as they do not bind column resin that strongly. However, quantitative experiments are necessary to figure out relative proportions of collected methylated peptides versus lost peptides.

Methylation to a single product has been successfully conducted for the commercially procured peptides FLEEVK, FLEENK, FLEETK, FLEEYK and FLEEHK. 2 $\mu \mathrm{g}$ of each peptide was combined into a solution, loaded onto an SCX column and methylated with diazomethane. Figure 7:6 shows a spectrum of these 5 peptides when they have been collected with MilliQ water after TrEnDi modification. Figure 7:6 shows full methylation of all peptides; the expected peaks and a fixed number of charges for these peptides are summarized in Table 7:4. 


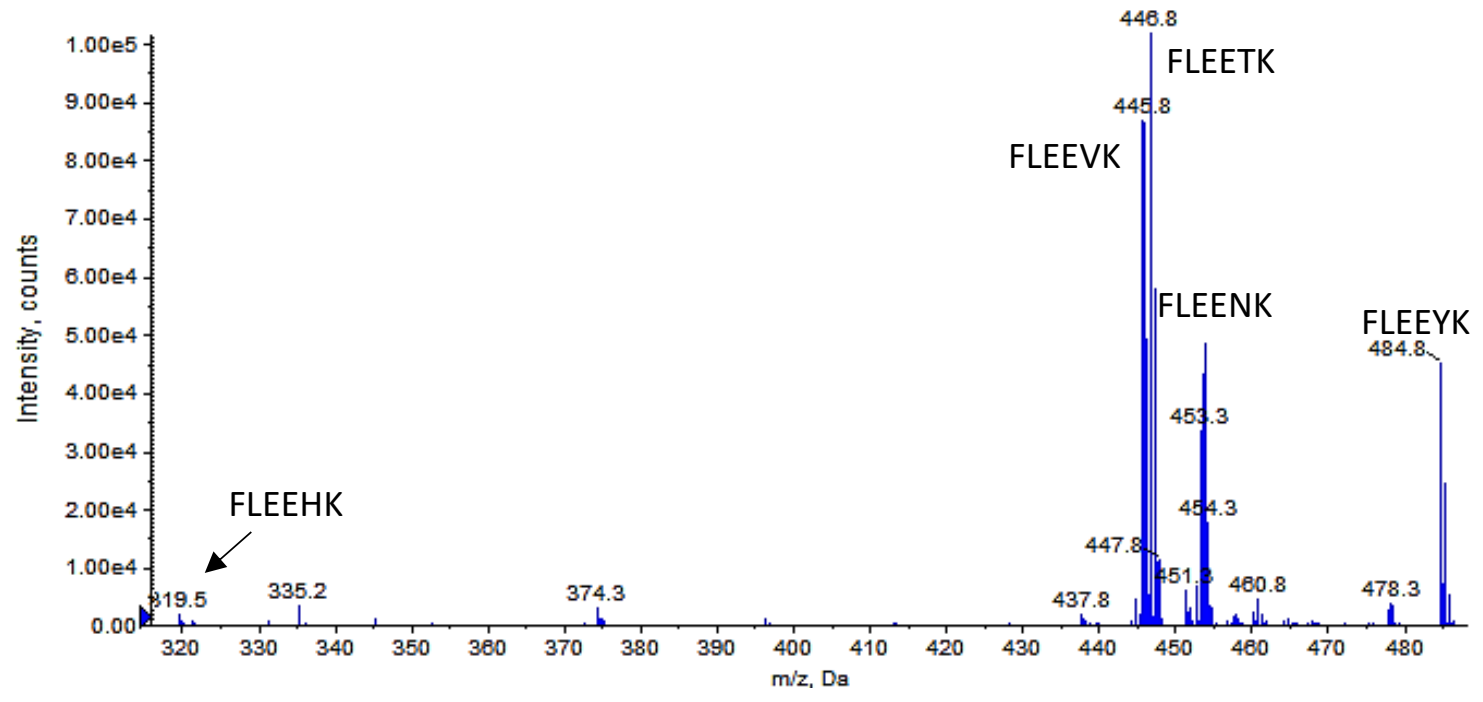

Figure 7:6. MS of 5 TrEnDi modified peptides eluted with $\mathrm{H}_{2} \mathrm{O}$.

Table 7:4. Commercial peptides with their neutral masses and $m / z$ ratios before and after TrEnDi modification. The table also shows a total number of methylations expected and obtained as well as a fixed number of positive charges.

\begin{tabular}{|c|c|c|c|c|c|c|}
\hline & \multicolumn{2}{|c|}{ Unmodified } & \multicolumn{2}{c|}{ TrEnDi modified } & \multicolumn{2}{c|}{ Methylation } \\
\hline Peptide & $\mathrm{Da}$ & $\mathrm{m} / \mathrm{z}$ & $\mathrm{Da}$ & $\mathrm{m} / \mathrm{z}$ & \# Me & \# fixed +c \\
\hline [FLEEVK] $^{2+}$ & 763.4 & 382.7 & 889.6 & 445.8 & 9 & 2 \\
\hline [FLEENK] $^{2+}$ & 778.4 & 390.2 & 904.6 & 453.3 & 9 & 2 \\
\hline [FLEETK] $^{2+}$ & 765.4 & 383.7 & 891.6 & 446.8 & 9 & 2 \\
\hline [FLEEYK] $^{2+}$ & 827.4 & 414.7 & 967.6 & 484.8 & 10 & 2 \\
\hline [FLEEHK] $^{3+}$ & 801.4 & 268.1 & 955.5 & 319.5 & 11 & 3 \\
\hline
\end{tabular}

It was also of interest to combine TrEnDi modifications with an HPLC separation to further enhance identification of proteins via MS. The 5 commercial TrEnDi modified peptides were loaded onto a new SCX column to ensure that TrEnDi modified peptides 
bind to it. As could be seen in Figure 7:7 there are no peaks of peptides observed from the loading solution which means that TrEnDi modified peptides bound to the resin.

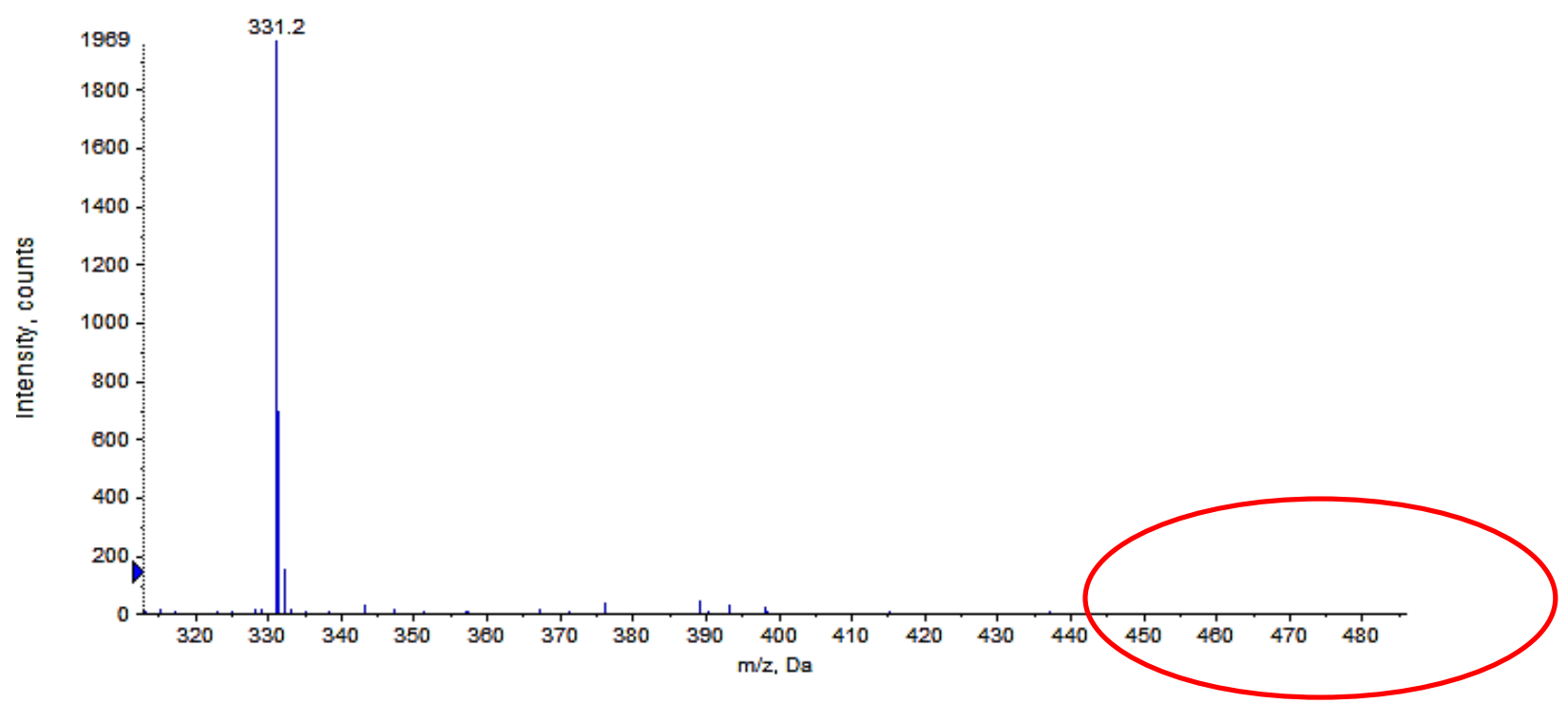

Figure 7:7. Loading TrEnDi modified peptides on a new SCX column.

Before trying to separate the 5 commercial TrEnDi modified peptides via HPLC, $200 \mathrm{mM}$ TEAA solution was tested as a potential eluent of these peptides, and the MS of the eluent solution is shown in Figure 7:8. As can be observed, TEAA showed promising results to be used as HPLC eluent for the separation of TrEnDi modified peptides. 


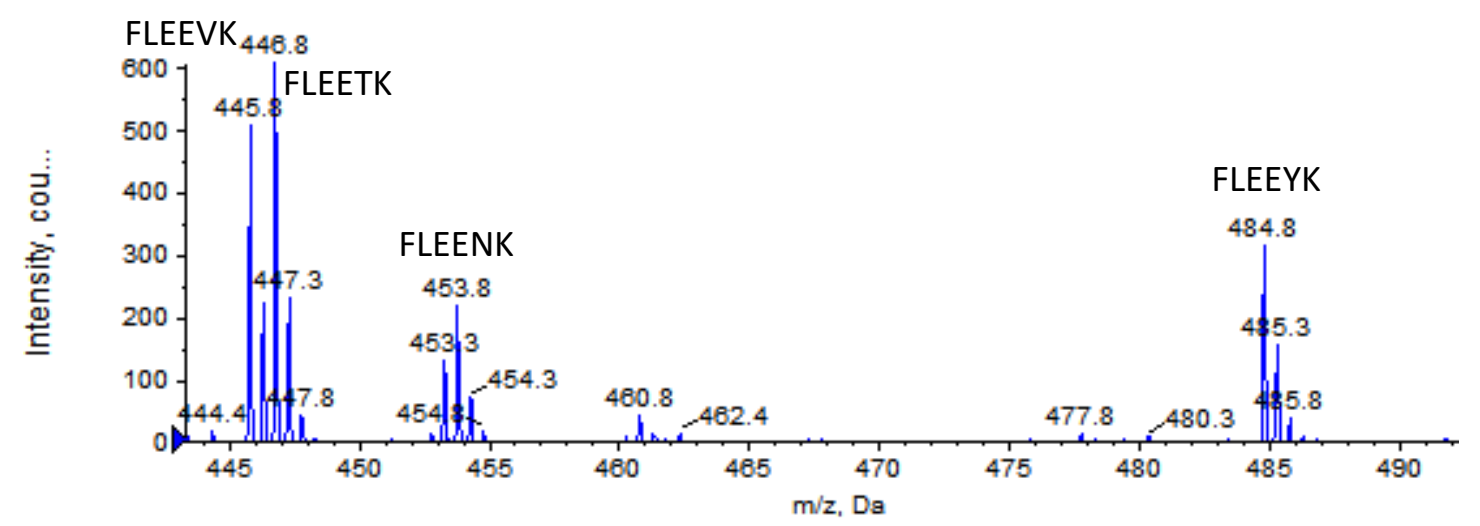

Figure 7:8. Eluting TrEnDi modified peptides with 200 mM TEAA solution. FLEEHK appears in a lot smaller intensity compared to the other peptides (not shown here).

After methylating a new batch of 5 commercial TrEnDi modified peptides on an SCX column, this column was attached in tandem with second SCX column and an LCMS run was conducted with a linear gradient of solvent $A(0.1 \%$ formic acid (FA) in $50 \%$ acetonitrile ( $\mathrm{ACN}$ ) and 50\% MilliQ $\mathrm{H}_{2} \mathrm{O}$ ) to $100 \%$ solvent $\mathrm{B}(200 \mathrm{mM}$ TEAA in $100 \%$ MilliQ $\mathrm{H}_{2} \mathrm{O}$ ) over 30 minutes. The result of LC Q-TOF experiment is shown in Figure 7:9. 5 extracted-ion chromatograms (EIC or XIC) could be seen in Figure 7:9, one for each peptide. This preliminary result shows that aqueous TEAA can be used as potential eluent of peptides via SCX chromatography and that TrEnDi modified peptides could be separated and identified via HPLC MS. 


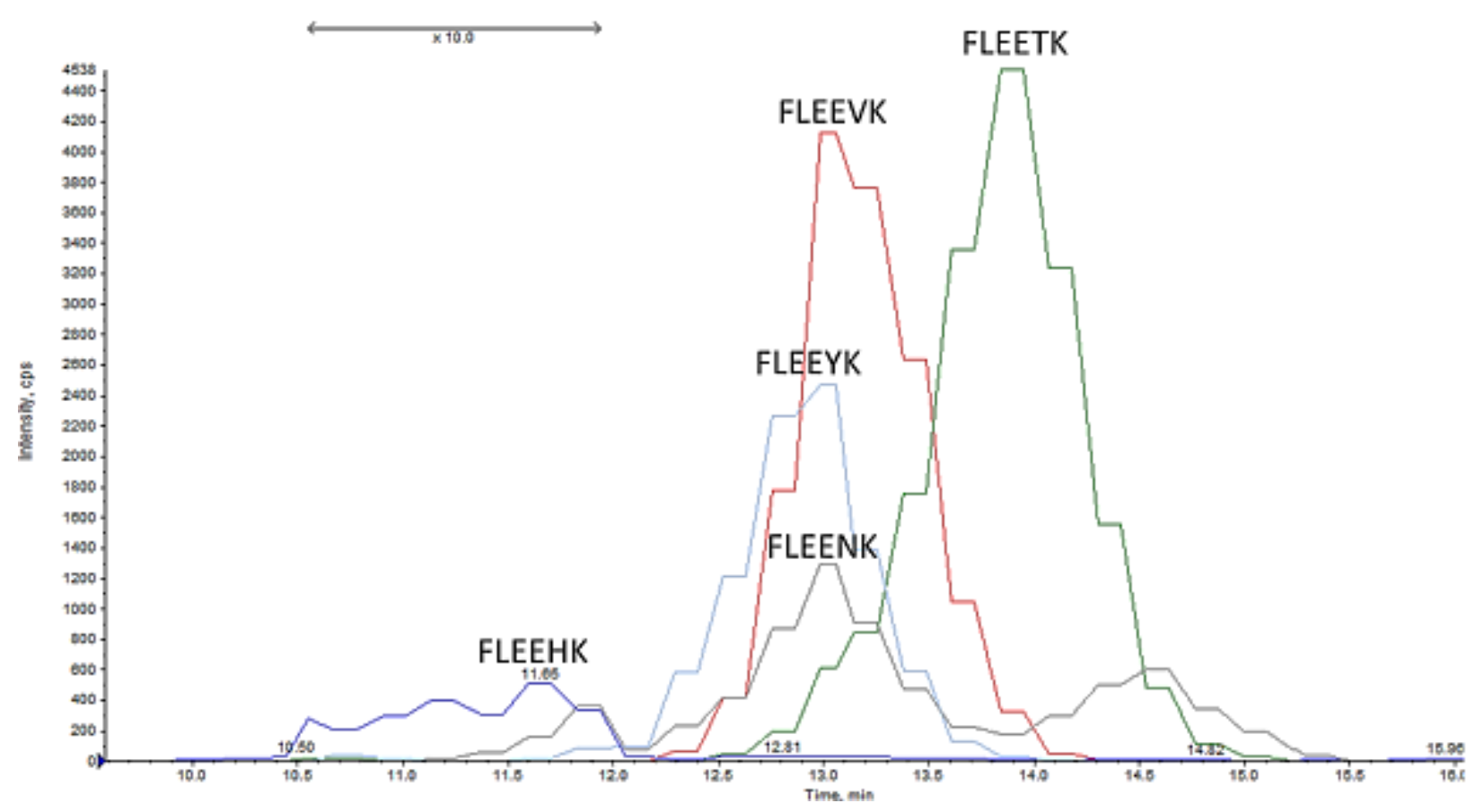

Figure 7:9. Eluting TrEnDi modified peptides over 30 minutes using a 0-200 mM TEAA gradient.

\subsubsection{TrEnDi to unify the charge state on methylated peptides}

Methylation is a type of post-translational modification, which is common in histone proteins. Core histone proteins form an octamer histone around which deoxyribonucleic acid (DNA) molecule wraps itself 5 . Together histone octamers and DNA form a DNAhistone complex called chromatin. Histone octamers are necessary; they allow DNA molecule to compact tightly, so that large DNA molecules could fit into cell nucleus (human cell has about 1.8 meters of $\mathrm{DNA}^{6}$ ), and they play an important part in gene regulation ${ }^{7}$. Methylation is common in histones; this way they increase the hydrophobic histone environment which increases packing of DNA molecule around it ${ }^{8}$. Peptides from histone protein have often quite complex MS spectra as these peptides are highly charged due to their abundant lysine and arginine amino acid content. It was of interest 
to see if TrEnDi could unify charge states on these peptides. For this purpose, two histone peptides were chosen for the study and will be discussed in the following paragraphs.

Experiments were conducted on histone peptides (ARTKQTARK and QTARKSTGGK) which intrinsically have varying numbers of charges on them in ESI-MS. ARTKQTARK may have 5 charges on it ( $\mathrm{N}$-terminus, $\mathrm{R}$ and $\mathrm{K}$ amino acids) and QTARKSTGGK may have 4 charges on it (N-terminus, $\mathrm{R}$ and $\mathrm{K}$ amino acids). In these peptides, the lysine amino acids are underlined because they were obtained with various natural degrees of methylation (0-3 methyl groups). In MS, the challenge of analyzing these molecules is that their masses differ based on differential methylation and each species is spread over several charge states. For example, ARTKQTARK peptides are seen to have 2, 3, 4 and, to a lesser extent, 5 charges. QTARKSTGGK peptides are seen to have 2, 3 and, to a lesser extent, 4 charges. The goal of our experiment was to unify the charge state of these peptides while also elucidating the degree of native methylation on each peptide. TrEnDi using ${ }^{13} \mathrm{C}$-labelled diazomethane was done on these molecules using the same procedure described above, and the results are summarized in Tables $7: 5$ and $7: 6$ for each peptide.

Table 7:5. ARTKKQTARK peptides in MS spectra before and after TrEnDi.

\begin{tabular}{|c|c|c|c|c|c|c|c|}
\hline ARTKQTARK & $\begin{array}{c}\mathrm{m} / \mathrm{z} \text { with } \\
\mathbf{2} \text { charges }\end{array}$ & $\begin{array}{c}\mathrm{m} / \mathrm{z} \text { with } \\
\mathbf{3} \text { charges }\end{array}$ & $\begin{array}{c}\mathrm{m} / \mathrm{z} \text { with } \\
4 \text { charges }\end{array}$ & $\begin{array}{c}\mathrm{m} / \mathrm{z} \text { with } \\
\mathbf{5} \text { charges }\end{array}$ & $\begin{array}{c}\text { TrEnDi } \\
\text { added \# } \\
\text { Me }\end{array}$ & $\begin{array}{c}{ }^{12} \mathrm{C} \\
\text { TrEnDi }\end{array}$ & $\begin{array}{c}{ }^{13} \mathrm{C} \\
\text { TrEnDi }\end{array}$ \\
\hline$\underline{\mathrm{KMe}}_{0}$ & 530.3 & 353.9 & 265.6 & 212.7 & 10 & 240.8 & 242.8 \\
\hline$\underline{\mathrm{KMe}}_{1}$ & 537.3 & 358.5 & 269.2 & 215.5 & 9 & 240.8 & 242.6 \\
\hline$\underline{\mathrm{KMe}}_{2}$ & 544.3 & 363.2 & 272.6 & 218.3 & 8 & 240.8 & 242.4 \\
\hline$\underline{\mathrm{KMe}}_{3}$ & 551.3 & 367.9 & 274.2 & 221.1 & 7 & 240.8 & 242.2 \\
\hline
\end{tabular}


Table 7:6. QTARKSTTGGK peptides in MS spectra before and after TrEnDi.

\begin{tabular}{|c|c|c|c|c|c|c|}
\hline QTARKSTGGK & $\begin{array}{c}\mathrm{m} / \mathrm{z} \text { with 2 } \\
\text { charges }\end{array}$ & $\begin{array}{c}\mathrm{m} / \mathrm{z} \text { with 3 } \\
\text { charges }\end{array}$ & $\begin{array}{c}\mathrm{m} / \mathrm{z} \text { with 4 } \\
\text { charges }\end{array}$ & $\begin{array}{c}\text { TrEnDi } \\
\text { added \# Me }\end{array}$ & ${ }^{12}$ C TrEnDi & ${ }^{13}$ C TrEnDi \\
\hline$\underline{\mathrm{KMe}}_{0}$ & 517.2 & 345.2 & 259.1 & 10 & 294.2 & 296.7 \\
\hline$\underline{\mathrm{KMe}}_{1}$ & 524.2 & 349.8 & 262.6 & 9 & 294.2 & 296.4 \\
\hline$\underline{\mathrm{KMe}}_{2}$ & 531.2 & 354.5 & 266.1 & 8 & 294.2 & 296.2 \\
\hline $\mathrm{KMe}_{3}$ & 538.2 & 359.2 & 269.6 & 7 & 294.2 & 295.9 \\
\hline
\end{tabular}

The spectra for unmodified and modified ARTKKQTARK and QTARKKSTGGK are shown in Figures 7:10-7:18. It could be observed that spectra for unmodified methylated histone looks quite complex. For ARTKQTARK, triply and quadruply charged molecules appear with the highest intensity, while doubly charged have a small intensity and quintuply charged molecules are almost not observed. For QTARKSSTGGK triply charged molecules appear with the highest intensity, doubly charged molecules are seen quite well, and quadruply charged molecules are almost not observed. After ${ }^{13} \mathrm{C} \operatorname{TrEnDi}\left(\mathrm{as}^{12} \mathrm{C}\right.$ TrEnDi cannot differentiate between the native and TrEnDi methylations), ARTKGQTARK peptides are observed to be mostly quintuply charged (very low abundance quadruply charged molecules can be observed) and QTARKSTTGGK are observed to only be quadruply charged. These results show that TrEnDi modification shows potential to unify the charge state of methylated peptides that are generally observed with multiple charge states. 


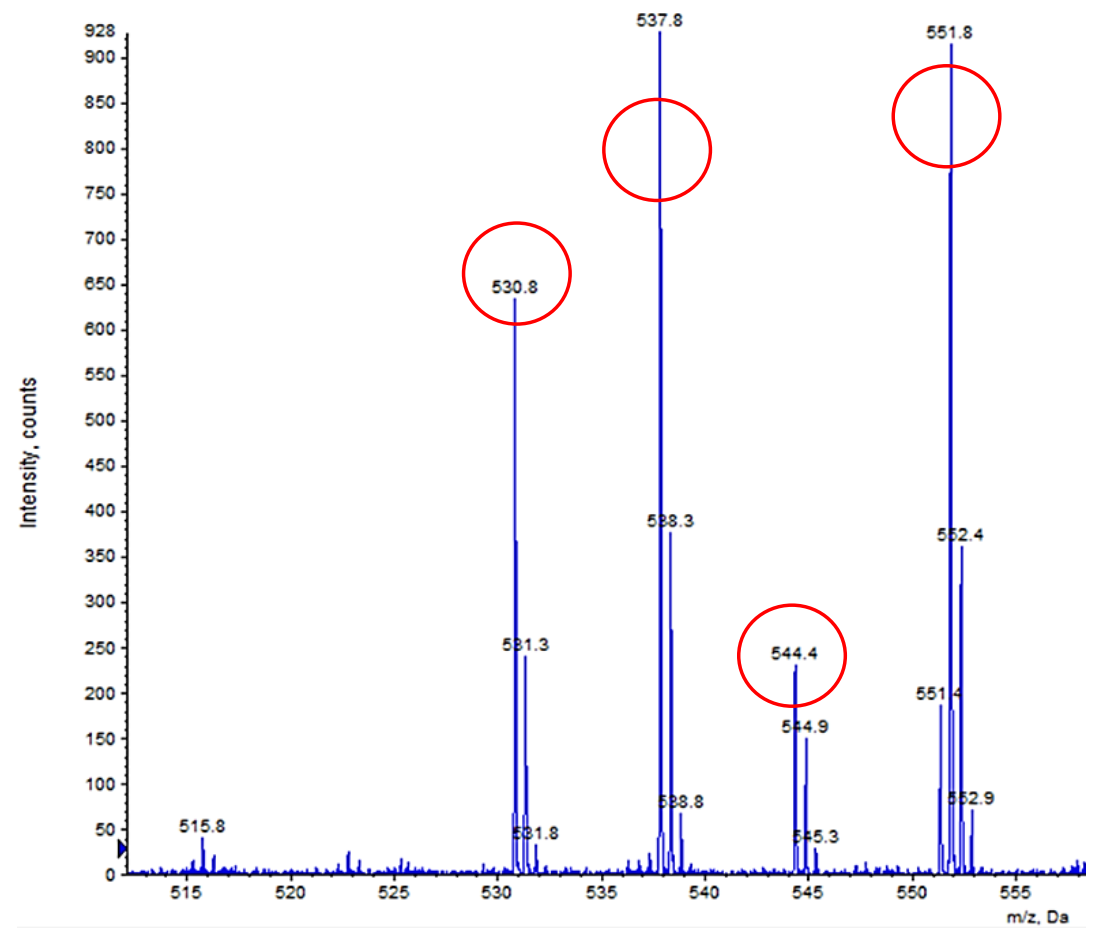

Figure 7:10. Unmodified $\mathrm{H}_{3} \mathrm{~K}_{4}$ histone peptides doubly charged. 


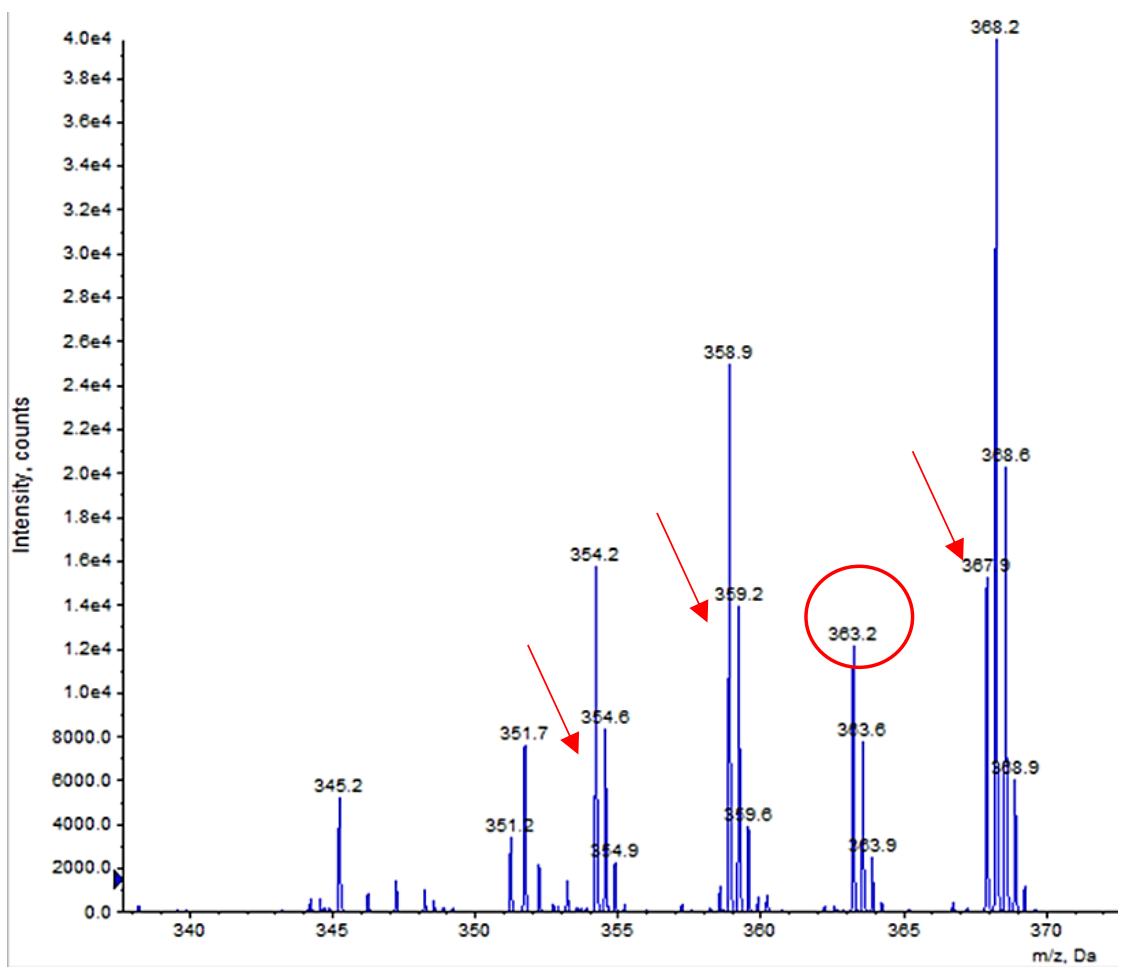

Figure 7:11. Unmodified $\mathrm{H}_{3} \mathrm{~K}_{4}$ histone peptides triply charged.

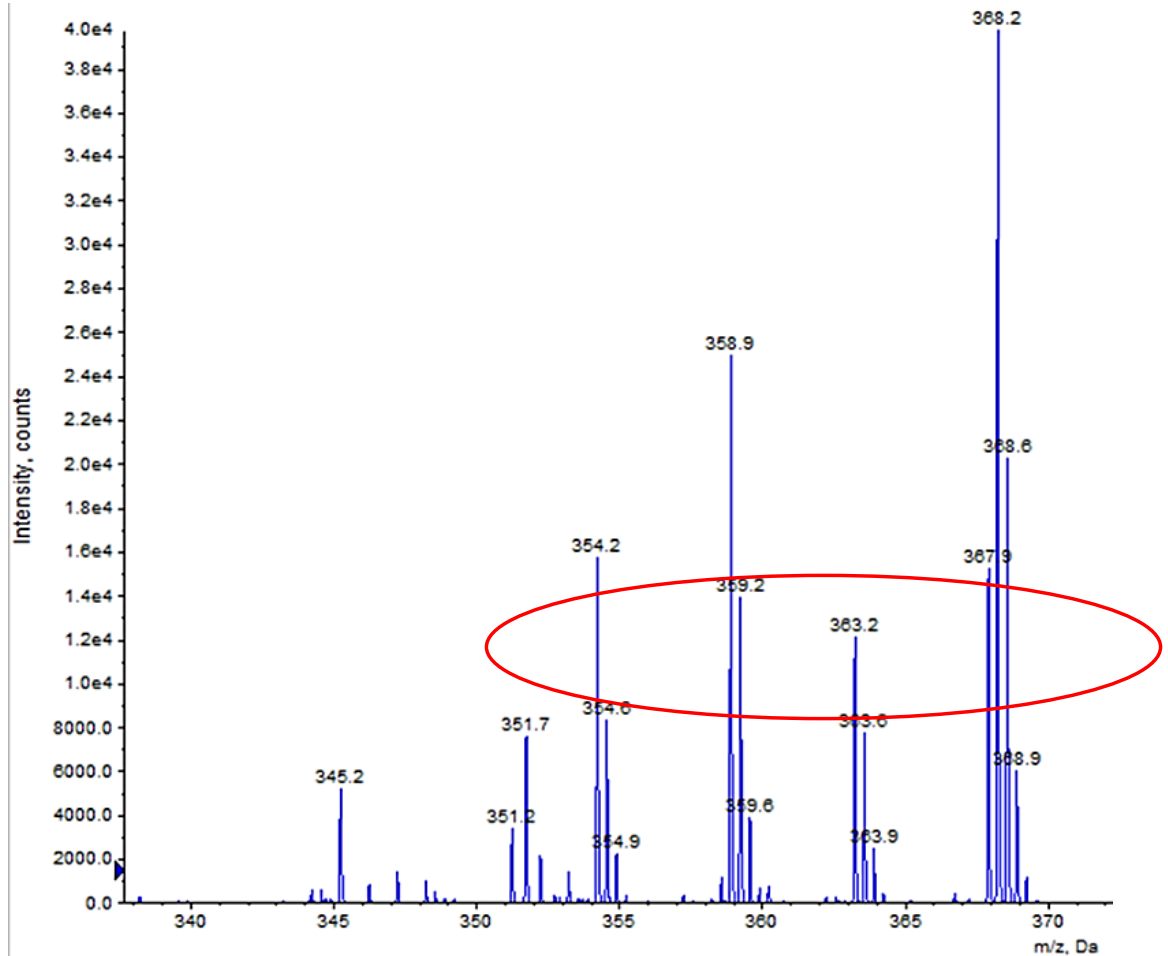

Figure 7:12. Unmodified $\mathrm{H}_{3} K_{4}$ histone peptides quadruply charged. 


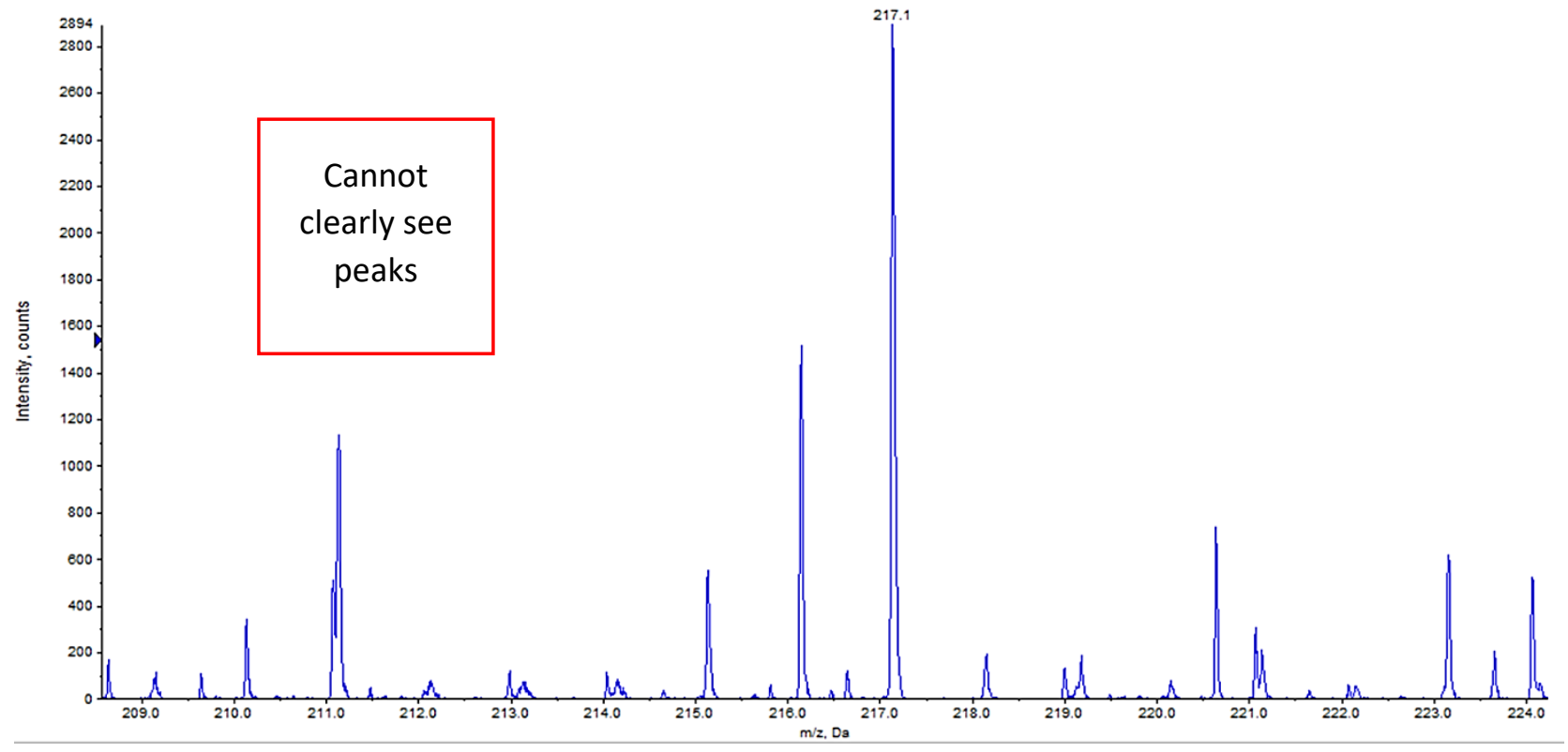

Figure 7:13. Unmodified $\mathrm{H}_{3} \mathrm{~K}_{4}$ histone peptides quintuply charged.

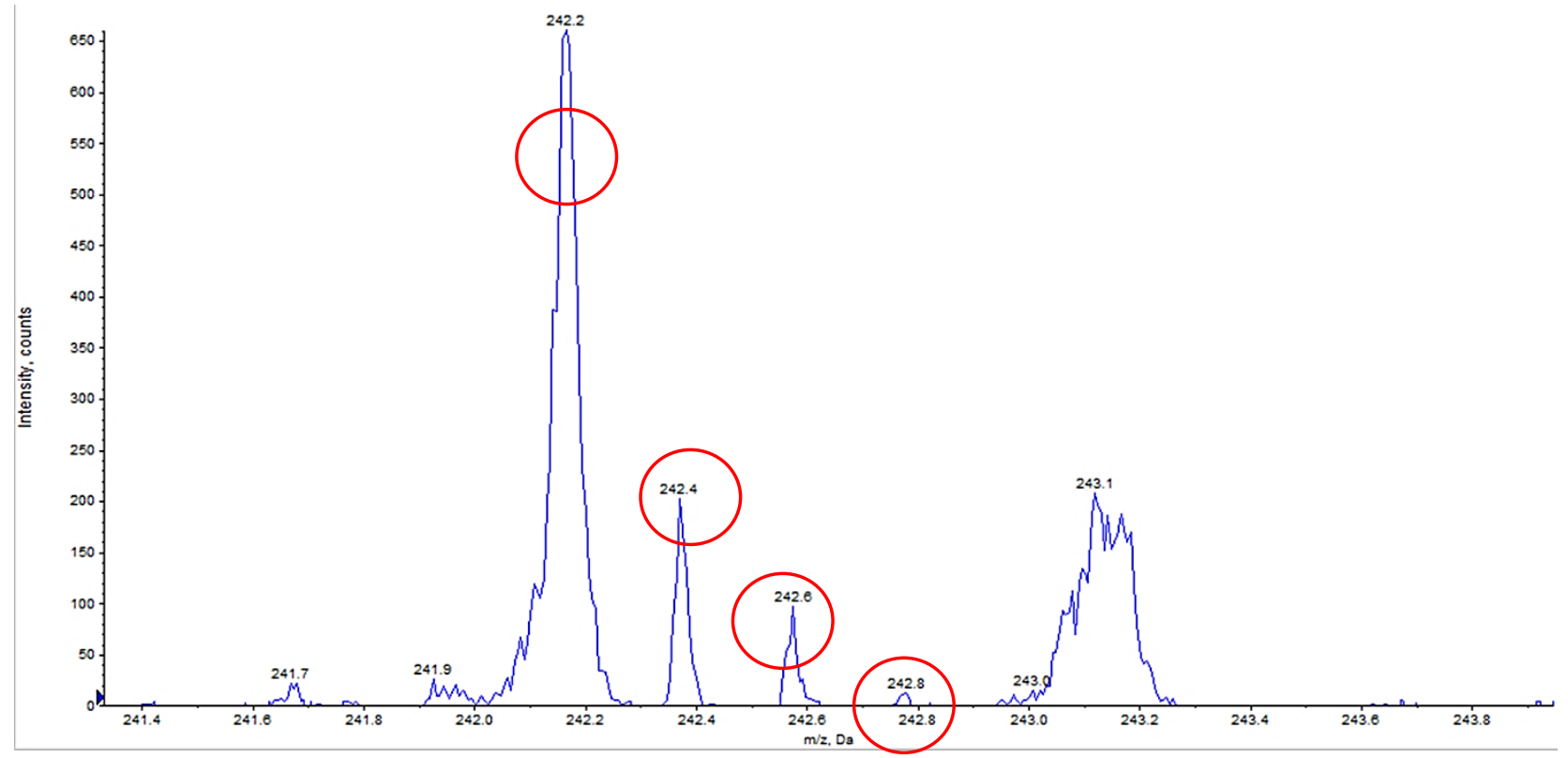

Figure 7:14. TrEnDi modified $\mathrm{H}_{3} \mathrm{~K}_{4}$ histone peptides quintuply charged. 


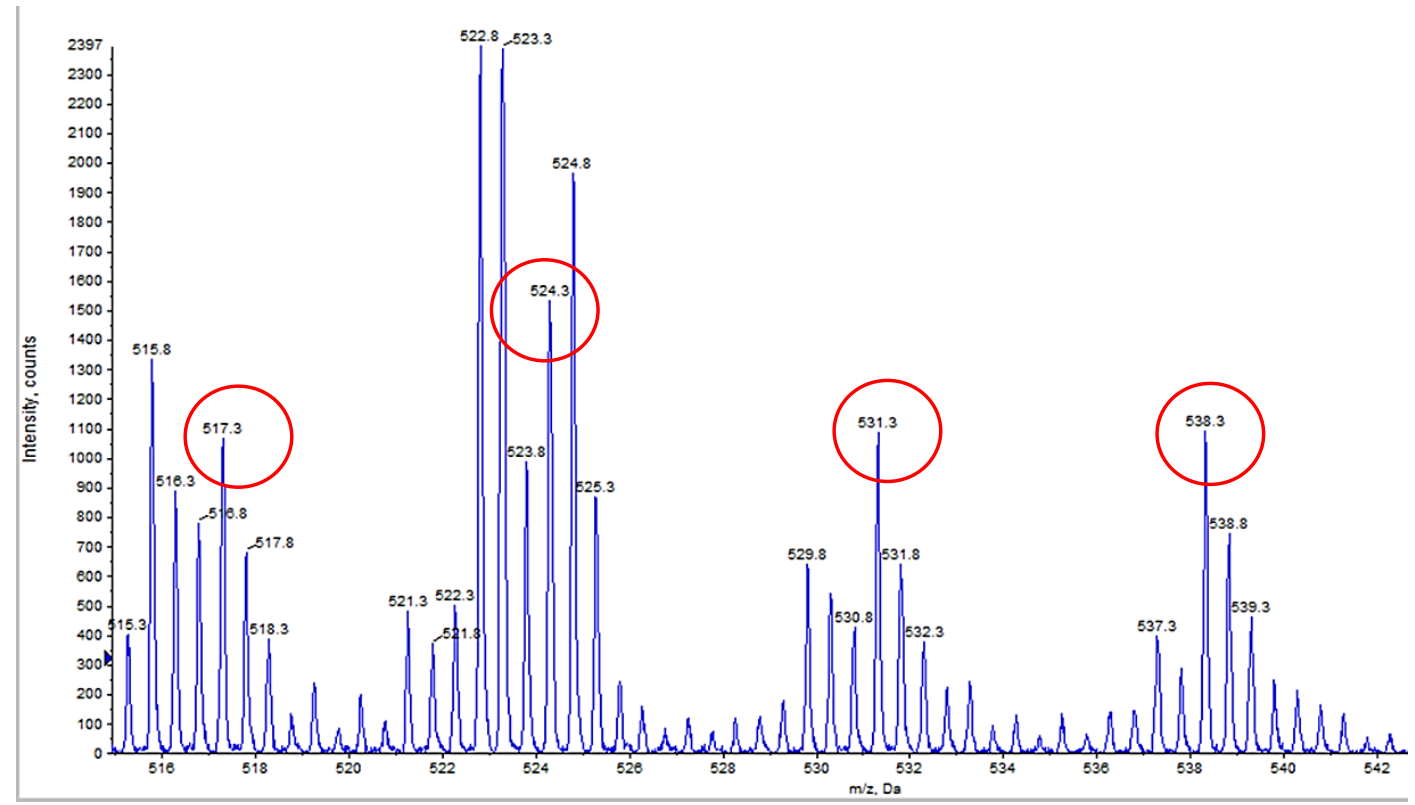

Figure 7:15. Unmodified $\mathrm{H}_{3} \mathrm{~K}_{9}$ histone peptides doubly charged.

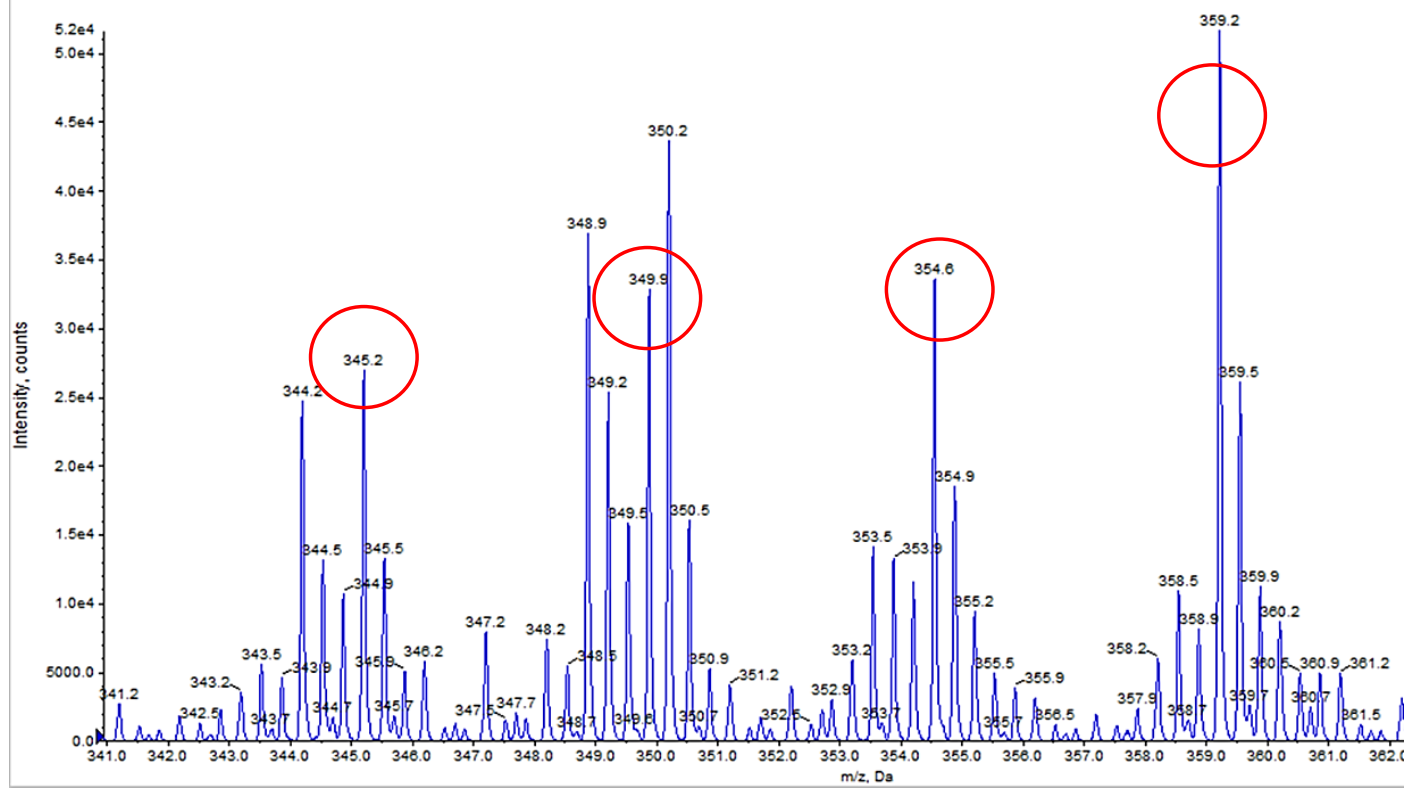

Figure 7:16. Unmodified $\mathrm{H}_{3} \mathrm{~K}_{9}$ histone peptides triply charged. 


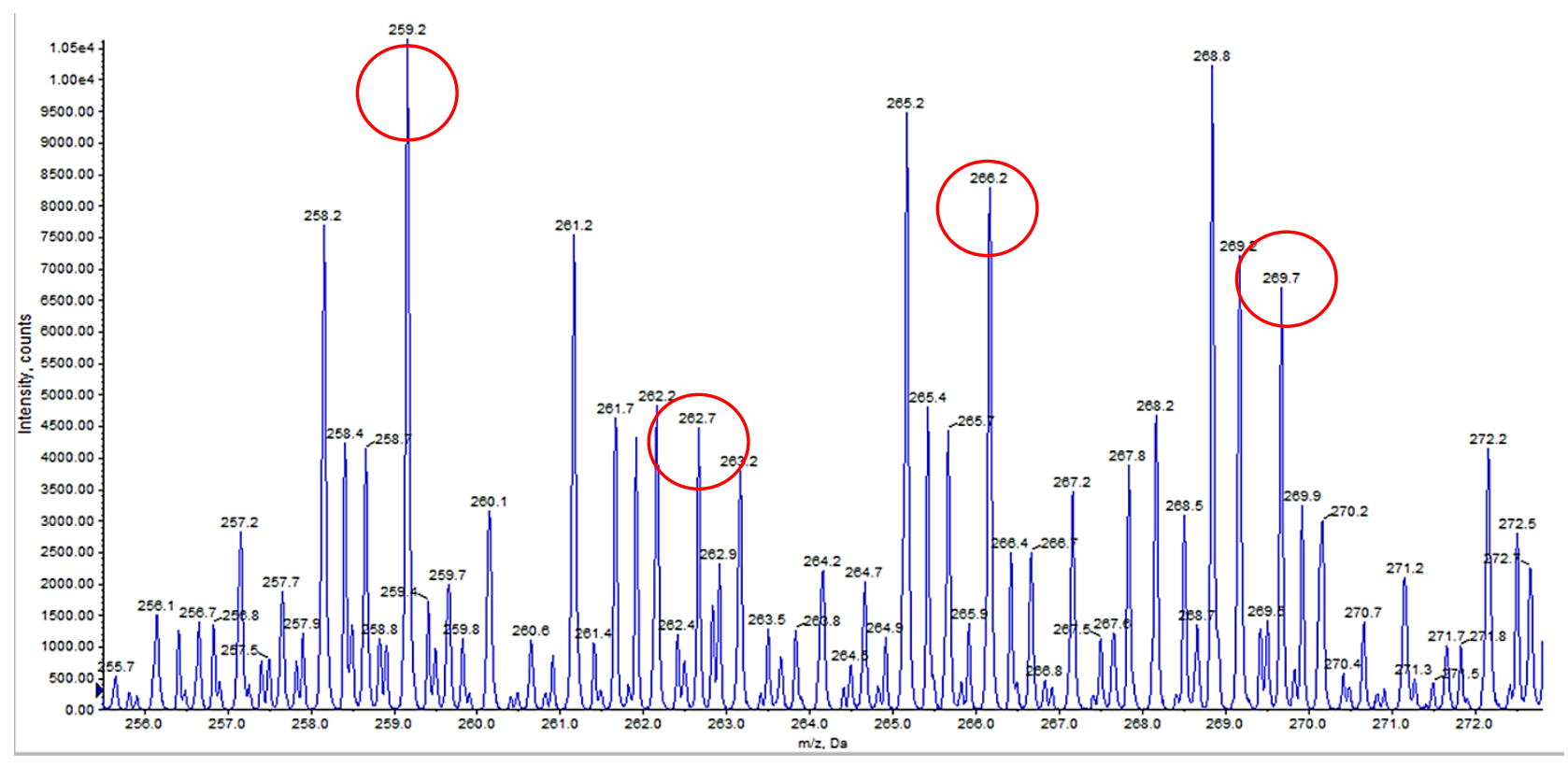

Figure 7:17. Unmodified $\mathrm{H}_{3} K_{9}$ histone peptides quadruply charged.

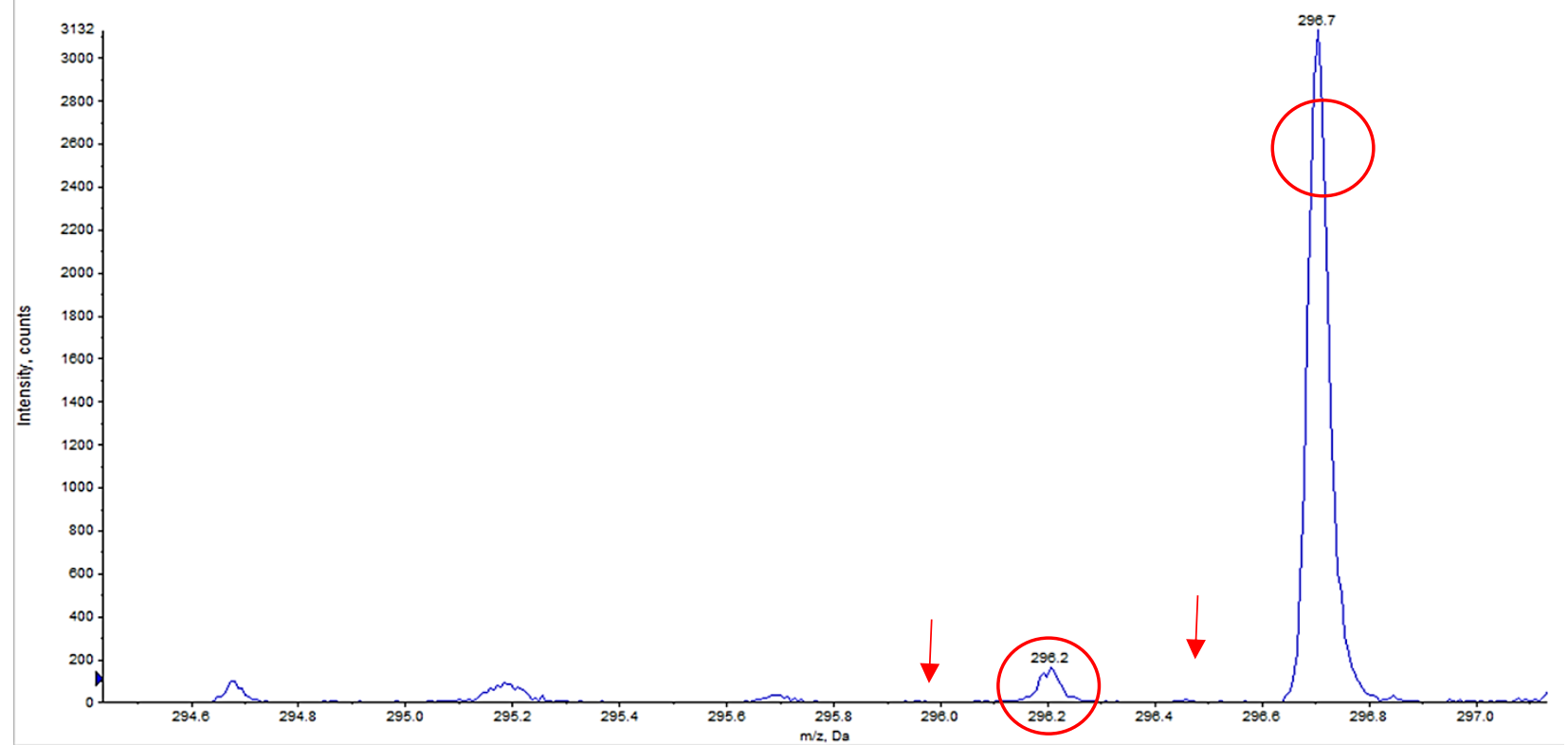

Figure 7:18. TrEnDi modified $\mathrm{H}_{3} \mathrm{~K}_{4}$ histone peptides quadruply charged. 


\subsubsection{Phosphorylated peptides using TrEnDi}

The addition of the phosphate groups to amino acid side chains on proteins is called phosphorylation. Phosphorylation via phosphoester bond formation is possible on the three amino acids; their structures and phosphorylated counterparts are shown in Figure 7:19. Phosphorylation of proteins changes the function and activity of proteins and abnormalities in protein phosphorylation are linked to many diseases ${ }^{9}$. One example of phosphorylation in a cellular regulatory mechanism is in the ATP-ADP (adenosine triphosphate - adenosine diphosphate) cycle where protein kinases take off phosphates from ATP and transfer them to amino acids, and protein phosphatases remove phosphate groups from proteins ${ }^{10}$.

Phosphorylated peptides are hydrophilic which make them difficult to separate on C18 reversed phase chromatography. Experiments were conducted to modify phosphorylated peptides via TrEnDi to see if they would exhibit any unique spectral enhancements via MS. The three phosphorylated peptides that were chosen for experiments were: FLEEpYK, FLEEpSK, and FLEEpTK. The TrEnDi modification was carried out with the same protocol as with commercial peptides described above. We hypothesized that methylation would occur on the $-\mathrm{OH}$ groups circled with red in Figure 7:19. The MS and MS/MS spectra were collected for three phosphorylated peptides after TrEnDi modification, shown in the following pages in Figures 7:20-7:25. It is important to note that the unmodified phosphorylated peptide spectra (not shown) reveal that these species appear as doubly and singly charged molecules. TrEnDi unifies the charge state to only doubly charged molecules. 


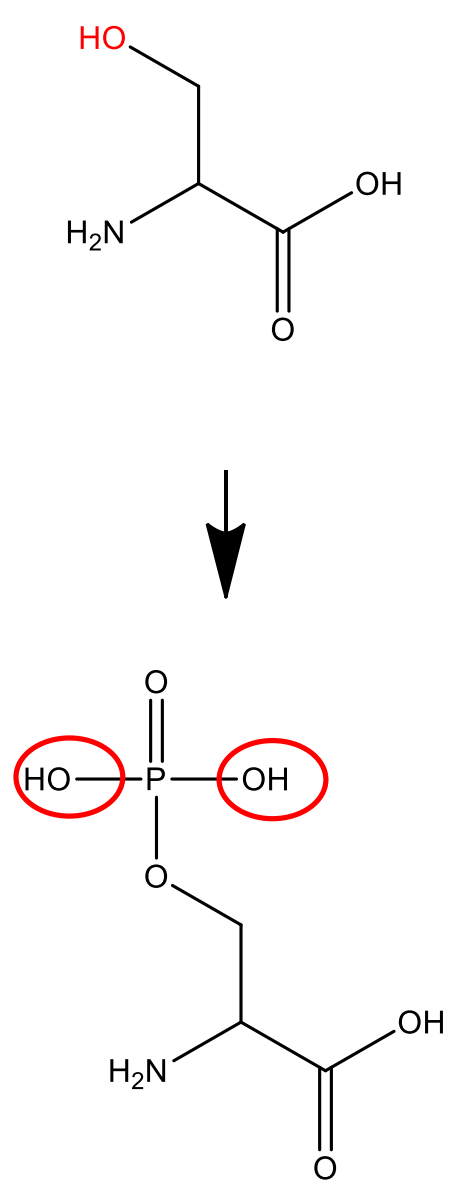

Serine (S)
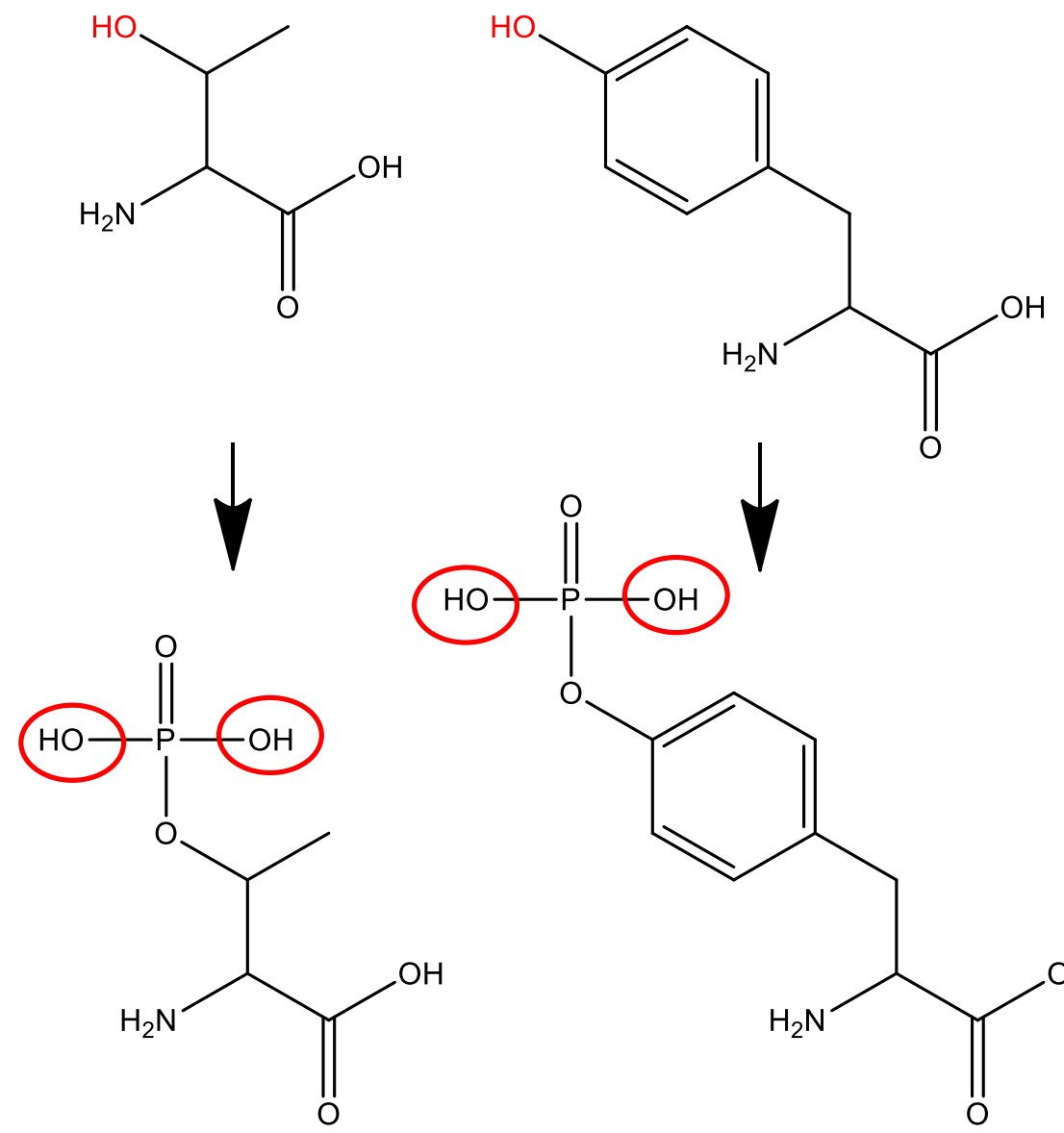

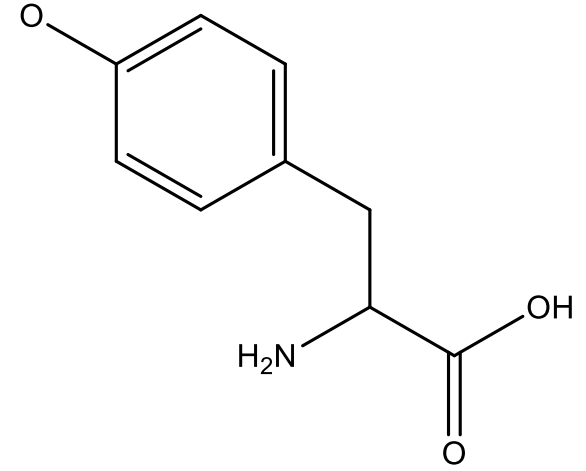

Tyrosine (Y)

Figure 7:19. Three amino acids that can be phosphorylated through the phosphoester bond formation. 


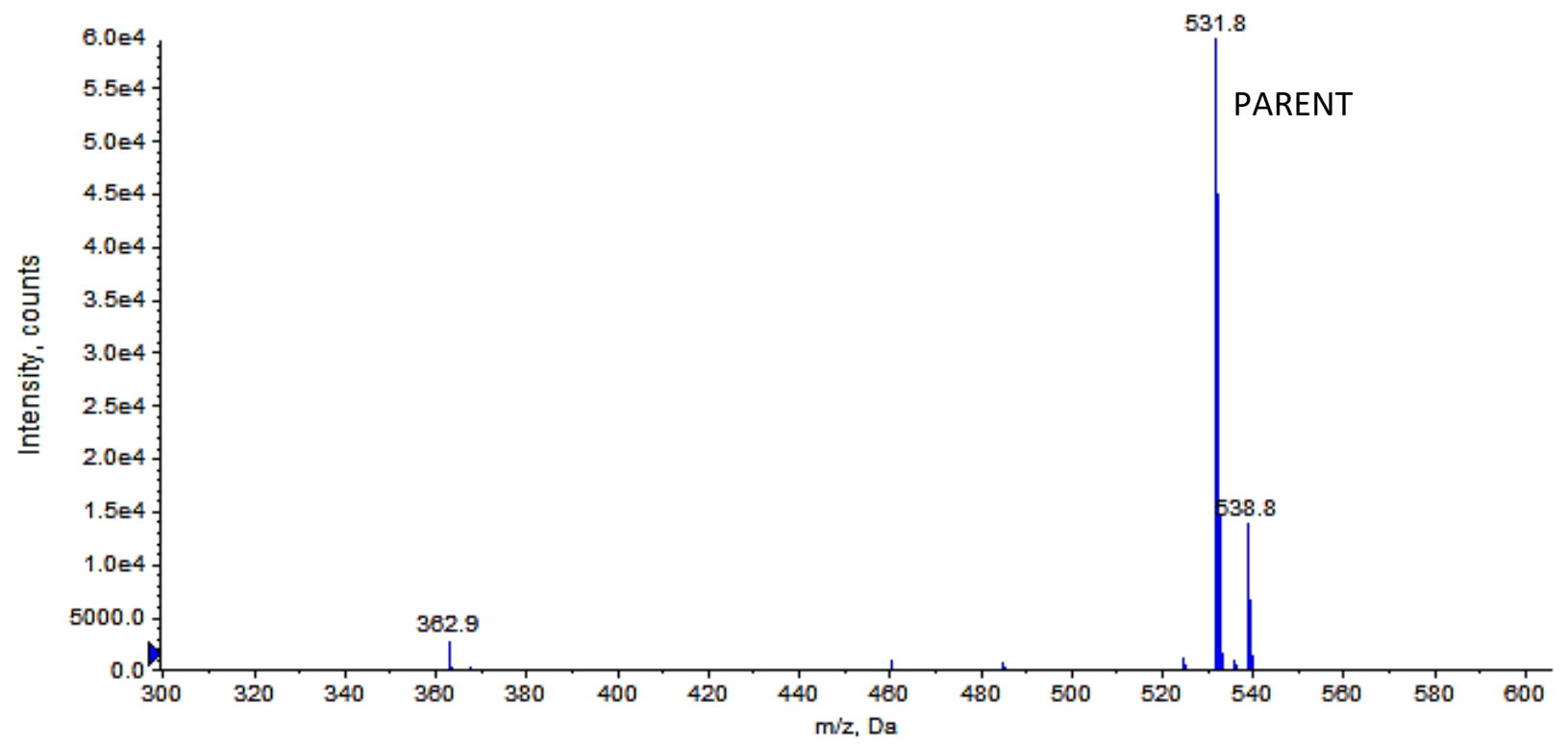

Figure 7:20. TrEnDi modified FLEEpYK.

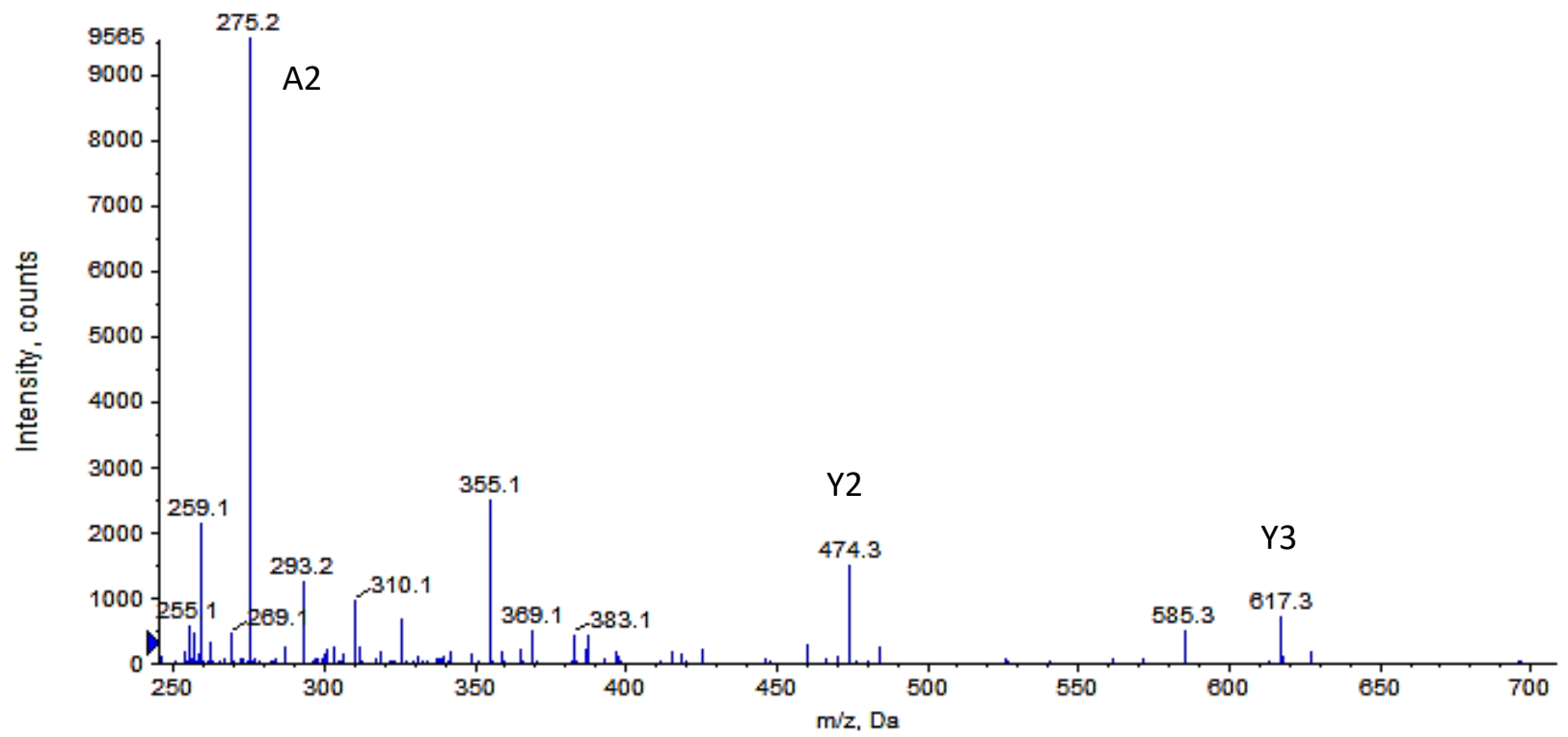

Figure 7:21. TrEnDi modified FLEEPYK - MS/MS 531. 


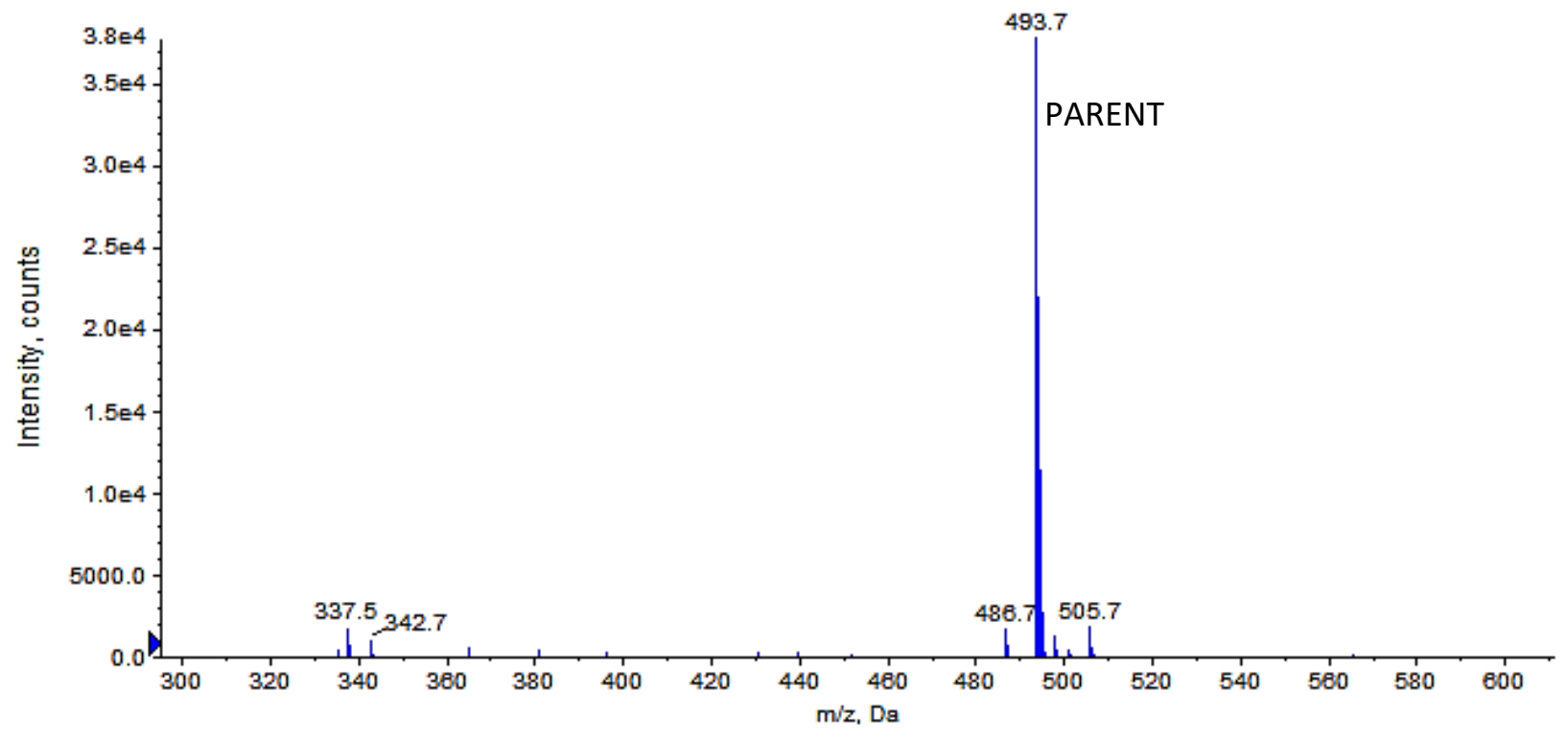

Figure 7:22. TrEnDi modified FLEEpSK.

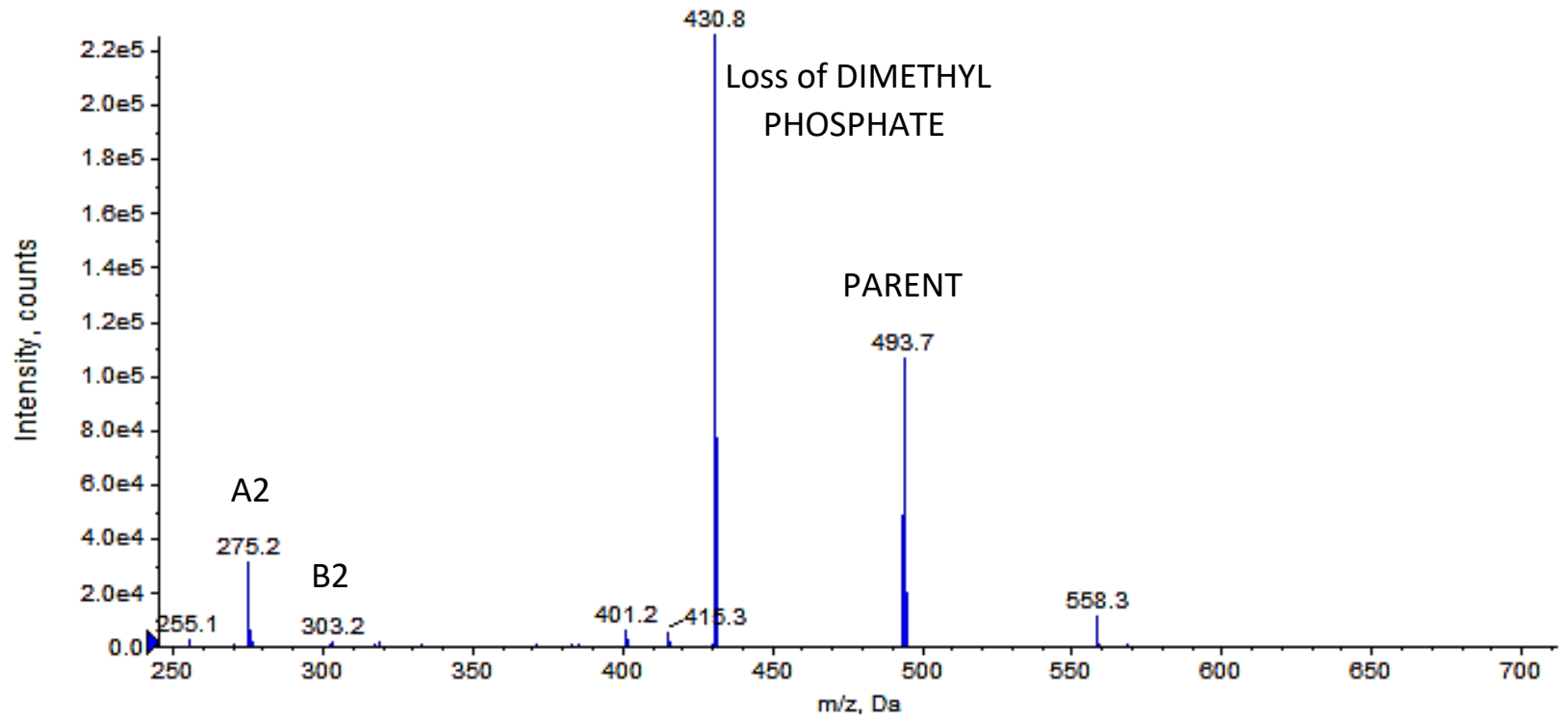

Figure 7:23. TrEnDi modified FLEEpSK - MS/MS 493. 


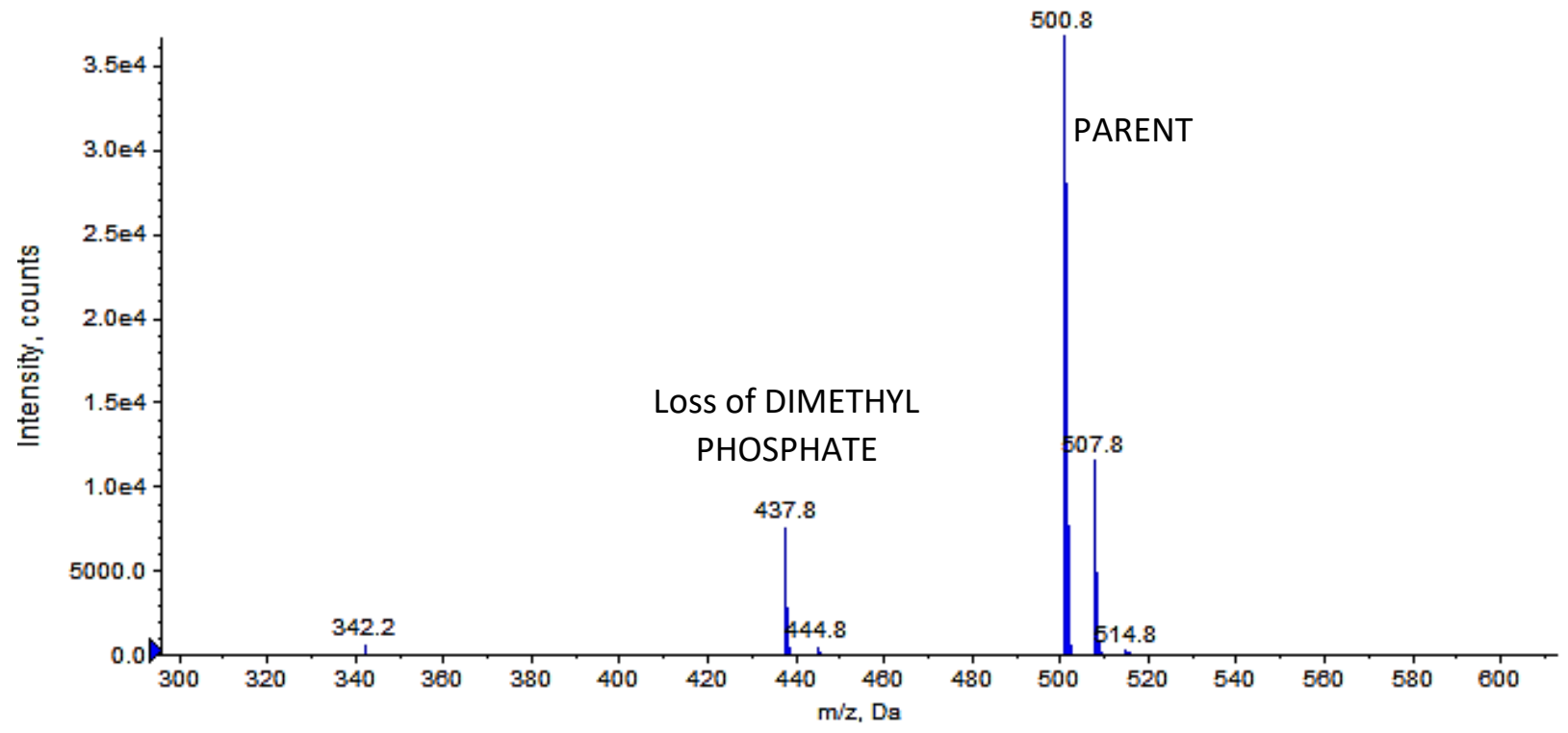

Figure 7:24. TrEnDi modified FLEEpTK.

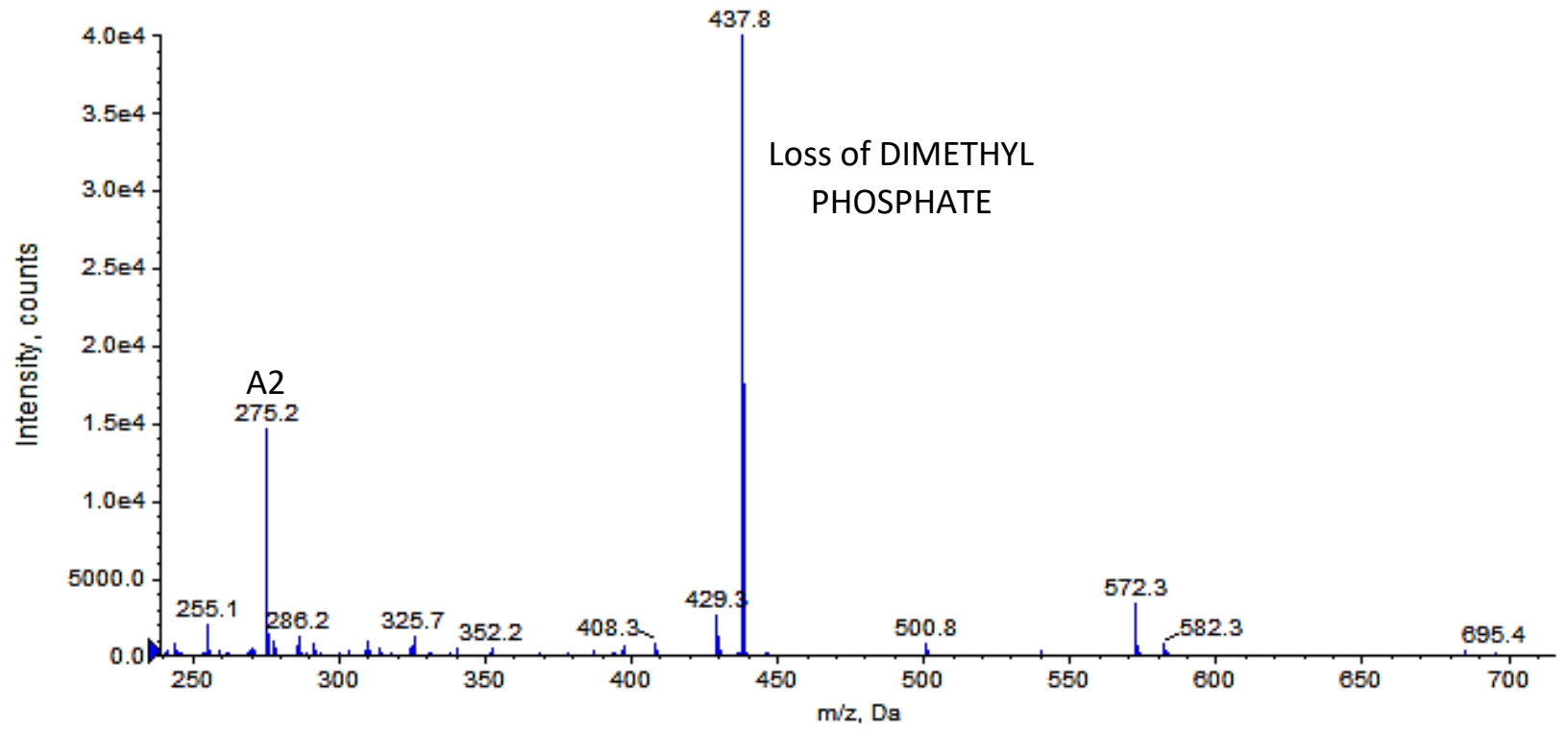

Figure 7:25. TrEnDi modified FLEEpTK - MS/MS 500. 
As it could be observed from Figures 7:20-7:25 that while FLEEpYK did not show any specific fragmentation patterns, FLEEpSK and FLEEpTK regularly showed a neutral loss of $126 \mathrm{Da}$, indicating that dimethyl phosphate is lost from the doubly charged peptides. This fragmentation pattern indicates a novel neutral loss scan that may be conducted on TrEnDi-modified phosphopeptides and may prove useful in the search for phosphopeptide-based biomarkers in future studies. In Figures 7:20 and 7:24 overmethylation peaks are seen that are $\mathrm{m} / \mathrm{z} 7$ higher compared to parent peaks, in Figure 7:22 small undermethylated peak is observed at $\mathrm{m} / \mathrm{z} 486.7$.

\subsubsection{Horse heart myoglobin}

After obtaining separation results of 5 commercial TrEnDi modified peptides, it was of interest to try this technique of separation and identification on a more complex sample. A commercial horse heart myoglobin (structure shown in Figure 2:14) was digested with trypsin. Peptides were modified via TrEnDi and were separated by various solvents on HPLC and analyzed via MS. These spectra were checked for sequence coverage using Mascot. Mascot reports the sequence coverage of each protein as a percentage; higher values translate into greater confidence in the protein identification.

The first separation of TrEnDi modified myoglobin peptides was done via SCX with $100 \%$ solvent $\mathrm{A}\left(0.1 \% \mathrm{FA}\right.$ in $50 \%$ acetonitrile $(\mathrm{ACN})$ and $50 \%$ MilliQ $\left.\mathrm{H}_{2} \mathrm{O}\right)$ going to $100 \%$ solvent $B\left(200 \mathrm{mM}\right.$ TEAA in $100 \%$ MilliQ $\left.\mathrm{H}_{2} \mathrm{O}\right)$ over 30 minutes. Unfortunately, good separation was not obtained. TEAA was not strong enough by itself to elute the TrEnDi modified peptides. ACN was added to solvent $B$, and it was noticed that TEAA and ACN are not miscible and form two layers. Then combinations of ACN with ammonium 
bicarbonate $(A B C)$ were tried for HPLC solvent $B$. In these cases, higher concentrations of $A B C$ led to clogging of the columns. As a result, none of those solvent combinations gave a good separation for TrEnDi modified myoglobin peptides.

To address these separations issues, reversed phase chromatography was employed. The diazomethane-treated SCX column was connected in tandem with a C18 column, and the following HPLC solvents were used: A $\left(0.1 \%\right.$ FA in MilliQ $\left.\mathrm{H}_{2} \mathrm{O}\right)$ and $B$ ( $0.1 \%$ FA in ACN). These conditions gave the best separation with a Mascot coverage of $28 \%$ from the injection of $4 \mu \mathrm{g}$ of peptides. To investigate the parameters of these experiments that would lead to the highest amount of sequence coverage, smaller amounts of TrEnDi modified myoglobin peptides were analyzed with varying amounts of diazomethane solution. Mascot sequence coverages varied between $11 \%-62 \%$ in different experiments. In every case, different peptides were identified in the different runs, and it was difficult to obtain consistent results. In addition to this, a great deal of variation in the degree of methylation was observed in the spectra. The best result obtained was $62 \%$ sequence coverage, obtained when $4 \mu \mathrm{g}$ of $\mathrm{TrEnDi}$ modified myoglobin peptides were reacted with $250 \mu \mathrm{L}$ diazomethane solution (approximately 0.2 M); this result is shown in Figure 7:26. 


\section{TrEnDiMyoglobin_methylatedwith250uL_in-line.mgf}

Protein sequence coverage: $62 \%$

Matched peptides shown in bold red.

1 GLSDGEWQQV LNVWGKVEAD IAGHOQEVLI RLPTGHPETL EKFDKFKHLK

51 TEAEMRASED LKKHGTVVLT ALGGILKKKK HHEAELKPLA QSYATKHKIP

101 IKYLEFISDA IIHVLHSKHP GDFGADAQGA WTRALELFRN DIAAKYKELO

$151 \mathrm{FQG}$

Figure 7:26. MASCOT sequence coverage for $4 \mu \mathrm{g}$ of TrEnDi modified myoglobin peptides methylated with $250 \mu \mathrm{L}$ of $0.2 \mathrm{M}$ diazomethane solution. Data obtained by Q-TOF instrument.

For comparison, MASCOT sequence coverage of $43 \%$ was obtained for trypsin digested horse heart myoglobin (no TrEnDi modification). Once the 6550 instrument became available to use, the best MASCOT sequence coverage for unmodified tryptically digested horse heart myoglobin was 98\% shown in Figure 7:27.

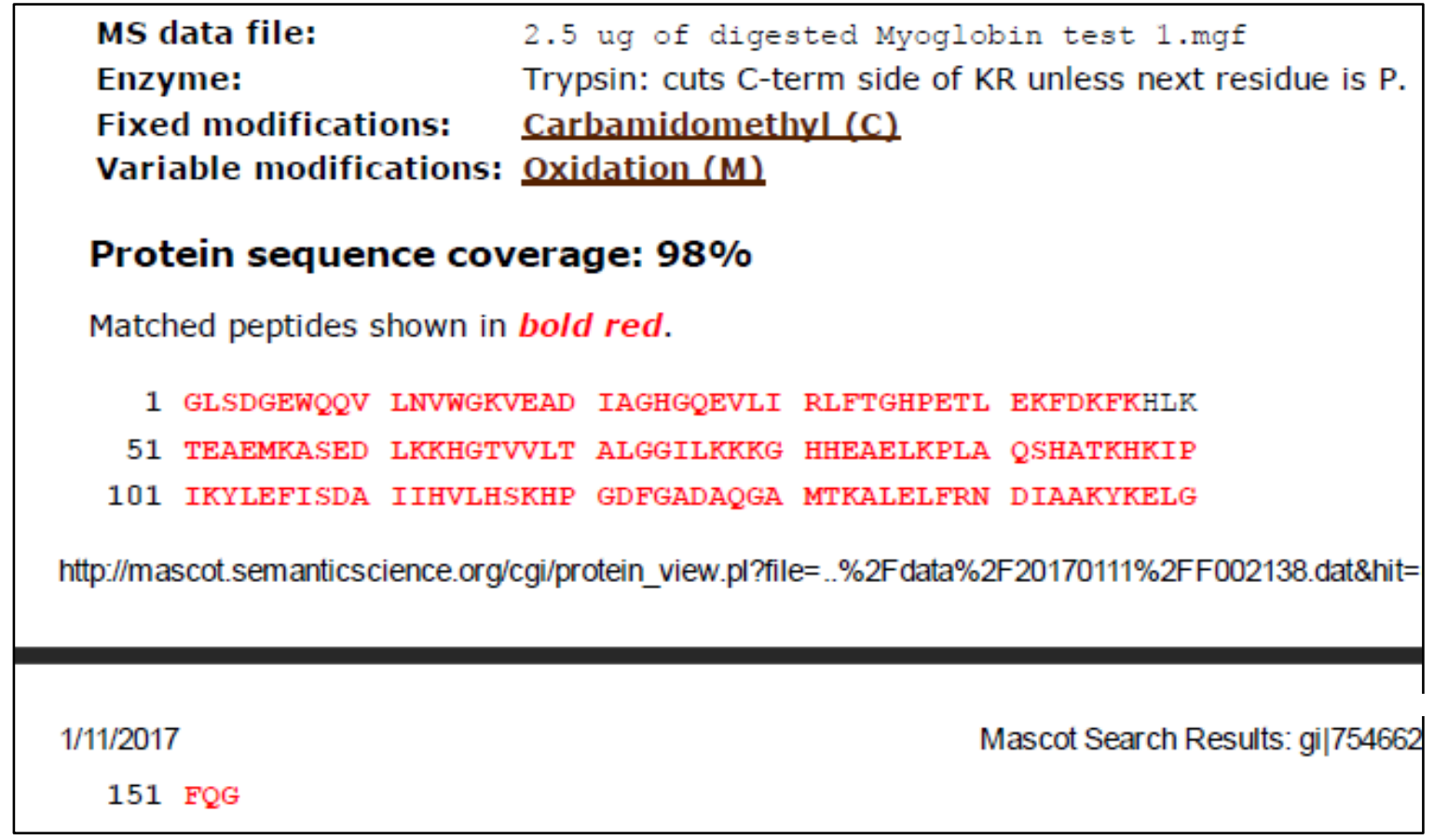

Figure 7:27. MASCOT sequence coverage for trypsin digested horse heart myoglobin. Data obtained by 6550 instrument. 


\subsection{Conclusions}

While there are still some challenges remaining with peptide TrEnDi derivatization due to difficulties controlling the rate and extent of methylation on peptides, there have been some patterns of success. Charge states have been unified to a single value and phosphopeptides fragment in a predictable and facile manner to yield a common neutral loss. As more powerful MS instruments emerge the need for enhancement techniques may decrease; however, the cost of new instrumentation is often prohibitive, and therefore enhancement techniques will continue to be useful tools to elucidate the complexity of various biological systems.

TrEnDi has shown to be useful in unifying charged species to a single charge state which has a potential in greatly reducing spectral complexity in MS. These results have also demonstrated that TrEnDi has the potential to monitor phosphopeptides/proteins in complex biological samples, which has been historically challenging via HPLC-MS. Due to highly hydrophilic nature of phosphopeptides, they are not easily retained on reversed phase stationary phases. However, TrEnDi-modified phosphopeptides are expected to have reduced hydrophilicity and be retained on non-polar stationary phases to a higher degree. TrEnDi also enhances the analysis of phosphopeptides via the introduction of a predominant neutral loss of $126 \mathrm{Da}$ via CID that can be easily monitored via neutral loss scanning.

TrEnDi has also show to be useful in unifying charged methylated histone peptides. The MS spectra obtained after TrEnDi modification was much simpler to interpret compared to unmodified spectra for those peptides. 


\subsection{References}

(1) Technical Bulletin. Sigma-Aldrich: St. Louis, MO, USA 2007.

(2) Pearce, M. Helv. Chim. Acta 1980, 63 (4), 887-891.

(3) Rendina, V. L.; Kingsbury, J. S. J. Org. Chem. 2012, 77 (2), 1181-1185.

(4) Wasslen, K. V.; Tan, L. H.; Manthorpe, J. M.; Smith, J. C. Anal. Chem. 2014, 86 (7), 3291-3299.

(5) Millar, T. Biochemistry explained. A practical guide to learning biochemistry; Overseas Publishers Association: Singapore, 2000.

(6) Widak, W. Molecular Biology: Not Only for Bioinforaticians; Widłak, W., Ed.; Lecture Notes in Computer Science; Springer Berlin Heidelberg: Berlin, Heidelberg, 2013; Vol. 8248.

(7) Verdone, L.; Caserta, M.; Mauro, E. Di. Biochem. Cell Biol. 2005, 83 (3), 344353.

(8) Rice, J. C.; Allis, C. D. Curr. Opin. Cell Biol. 2001, 13 (3), 263-273.

(9) Cohen, P. Eur. J. Biochem. 2001, 268 (19), 5001-5010.

(10) Ardito, F.; Giuliani, M.; Perrone, D.; Troiano, G.; Lo Muzio, L. Int. J. Mol. Med. 2017. 